\title{
Should Contemporary Density Functional Theory Methods be Used to Study the Thermodynamics of Radical Reactions?
}

Ekaterina I. Izgorodina, ${ }^{\dagger}$ David R. B. Brittain, Jennifer L. Hodgson, Elizabeth H.

Krenske, Ching Yeh Lin, Mansoor Namazian and Michelle L. Coote ${ }^{*}$

ARC Centre of Excellence in Free-Radical Chemistry and Biotechnology, Research

School of Chemistry, Australian National University, Canberra ACT 0200, Australia

mcoote@rsc.anu.edu.au

SUPPORTING INFORMATION

Current address: School of Chemistry, Monash University, Victoria 3800, Australia

* Corresponding author. Fax 6126125 0750. Email address: mcoote@rsc.anu.edu.au 


\section{TABLE S1: Geometries of the R•, X• and R-X species in Table 1.}

NOTE: All species had zero imaginary frequencies, as determined from frequency calculations at the B3-LYP/6-31G(d) level

\section{Me•}

$1 \backslash 1 \backslash G I N C-S C 81 \backslash F O p t \backslash U B 3 L Y P \backslash 6-31 G(d) \backslash C 1 H 3(2) \backslash E X I 501 \backslash 06-J a n-2005 \backslash 0 \backslash \backslash \# P \quad G F$ INPUT UB3LYP/6-31G(D) SCF=TIGHT OPT=TIGHT FREQ MAXDISK=134217728\\CH3 radical optimization $\backslash \backslash 0,2 \backslash \mathrm{C}, 0 ., 0 ., 0 . \backslash \mathrm{H}, 0 .,-1.082752,0 . \backslash \mathrm{H},-0.937690738$, $0.541376,0 . \backslash \mathrm{H}, 0.937690738,0.541376,0 . \backslash \backslash$ Version=DEC-AXP-OSF/1-G0 3RevB. 0 $3 \backslash$ State $=2-\mathrm{A} 2 \mathrm{"} \backslash \mathrm{HF}=-39.8382922 \backslash \mathrm{S} 2=0.753765 \backslash \mathrm{S} 2-1=0 . \backslash \mathrm{S} 2 \mathrm{~A}=0.750007 \backslash \mathrm{RMSD}=4.9$ $00 e-09 \backslash \mathrm{RMSF}=9.710 e-08 \backslash \mathrm{Dipole}=0 ., 0 ., 0 . \backslash \mathrm{PG}=\mathrm{D} 03 \mathrm{H} \quad[\mathrm{O}(\mathrm{C} 1), 3 \mathrm{C} 2(\mathrm{H} 1)] \backslash \backslash$

\section{$\cdot \mathrm{CH}_{2} \mathrm{OH}$}

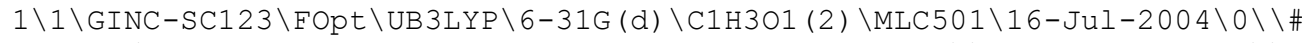

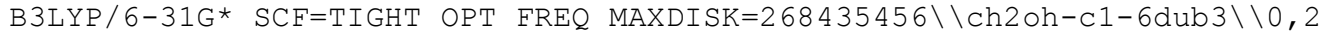
$\backslash \mathrm{H},-1.0942276995,0.7394861809,-0.0942454358 \backslash 0,-0.6716719027,-0.1240756$ $087,0.0265479373 \backslash \mathrm{C}, 0.6861955276,0.0279011485,-0.075297734 \backslash \mathrm{H}, 1.12604668$ $24,0.9793570076,0.2196810064 \backslash \mathrm{H}, 1.224383073,-0.8936452104,0.1139673354 \backslash$ $\backslash$ Version=DEC-AXP-OSF/1-G03RevB.03 \State $=2-A \backslash H F=-115.0520323 \backslash S 2=0.75293$ $1 \backslash \mathrm{S} 2-1=0 . \backslash \mathrm{S} 2 \mathrm{~A}=0.750006 \backslash \mathrm{RMSD}=6.164 \mathrm{e}-09 \backslash \mathrm{RMSF}=7.097 \mathrm{e}-05 \backslash \mathrm{Dipole}=-0.0182302$ $, 0.6010966,0.0823884 \backslash P G=C 01 \quad[X(\mathrm{C} 1 \mathrm{H} 301)] \backslash \backslash @$

\section{$\cdot \mathrm{CH}_{2} \mathrm{CN}$}

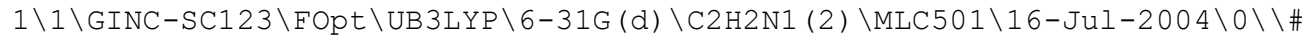

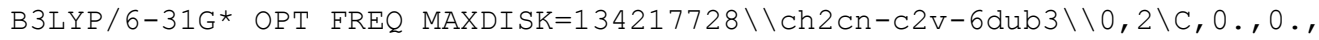
$0.1864058559 \backslash \mathrm{N}, 0 ., 0 ., 1.3633890519 \backslash \mathrm{C}, 0 ., 0 .,-1.1961782849 \backslash \mathrm{H}, 0 ., 0.9362690$ $64,-1.7425443946 \backslash \mathrm{H}, 0 .,-0.936269064,-1.7425443946 \backslash \backslash$ Version=DEC-AXP-OSF / $1-G 03 R e v B .03 \backslash$ State $=2-B 1 \backslash H F=-132.0946679 \backslash S 2=0.768772 \backslash S 2-1=0 . \backslash S 2 A=0.7501$ $44 \backslash \mathrm{RMSD}=5.076 \mathrm{e}-09 \backslash \mathrm{RMSF}=1.371 \mathrm{e}-04 \backslash \mathrm{Dipole}=0 ., 0 .,-1.3176858 \backslash \mathrm{PG}=\mathrm{C} 02 \mathrm{~V} \quad[\mathrm{C} 2(\mathrm{C}$ $1 \mathrm{C} 1 \mathrm{~N} 1), \mathrm{SGV}(\mathrm{H} 2)] \backslash \backslash \mathrm{C}$

\section{$\cdot \mathrm{CH}_{2} \mathbf{F}$}

$1 \backslash 1 \backslash G I N C-S C 123 \backslash F O p t \backslash U B 3 L Y P \backslash 6-31 G(d) \backslash C 1 H 2 F 1(2) \backslash M L C 501 \backslash 16-J u l-2004 \backslash 0 \backslash \backslash \#$

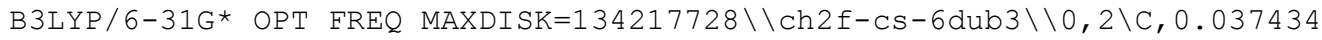
$0576,0.6566054142,0 . \backslash \mathrm{F}, 0.0198346227,-0.6860259304,0 . \backslash \mathrm{H},-0.2015579751,1$ $.1173004441,0.9530618991 \backslash \mathrm{H},-0.2015579751,1.1173004441,-0.9530618991 \backslash \backslash \mathrm{V}$ ersion $=\mathrm{DEC}-\mathrm{AXP}-\mathrm{OSF} / 1-\mathrm{GO} 3 \mathrm{RevB} .03 \backslash$ State $=2-\mathrm{A}^{\prime} \backslash \mathrm{HF}=-139.0642668 \backslash \mathrm{S} 2=0.753038$ $\backslash \mathrm{S} 2-1=0 . \backslash \mathrm{S} 2 \mathrm{~A}=0.750006 \backslash \mathrm{RMSD}=8.395 \mathrm{e}-10 \backslash \mathrm{RMSF}=6.939 \mathrm{e}-05 \backslash \mathrm{Dipole}=-0.1540613$, $0.465355,0 . \backslash P G=C S \quad[S G(C 1 F 1), X(H 2)] \backslash \backslash @$

\section{$\bullet \mathrm{CH}_{2} \mathrm{Ph}$}

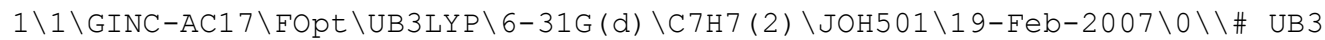
$\mathrm{LYP} / 6-31 \mathrm{G} * \mathrm{OPT} \mathrm{FREQ}=$ noraman maxdisk $=134217728 \backslash \backslash \mathrm{ch} 2 \mathrm{ph} 1 \mathrm{~s} \backslash \backslash 0,2 \backslash \mathrm{C}, 0.00000$ $00117,0 ., 0 . \backslash \mathrm{C}, 0.0000000117,0 ., 1.40687 \backslash \mathrm{C}, 0.0000000117,0 .,-2.83492 \backslash \mathrm{H}, 0.0$ $000000117,0 .,-3.92119 \backslash \mathrm{H}, 0.0000000117,-0.9280274624,1.9694437125 \backslash \mathrm{H}, 0.00$ $00000117,0.9280274624,1.9694437125 \backslash C, 0.0000000117,-1.2180774854,-0.742$ $9400194 \backslash \mathrm{C}, 0.0000000117,1.2180774854,-0.7429400194 \backslash \mathrm{C}, 0.0000000117,-1.21$ $20641953,-2.1288369739 \backslash \mathrm{C}, 0.0000000117,1.2120641953,-2.1288369739 \backslash \mathrm{H}, 0.0$ $000000117,-2.1606878954,-0.2009325066 \backslash \mathrm{H}, 0.0000000117,2.1606878954,-0.2$ $009325066 \backslash \mathrm{H}, 0.0000000117,-2.1540130532,-2.6714927354 \backslash \mathrm{H}, 0.0000000117,2$. $1540130532,-2.6714927354 \backslash \backslash$ Version=IA64L-G03RevD.01 \State $=2-A^{\prime} \backslash H F=-270$. $9151433 \backslash \mathrm{S} 2=0.783658 \backslash \mathrm{S} 2-1=0 . \backslash \mathrm{S} 2 \mathrm{~A}=0.750768 \backslash \mathrm{RMSD}=7.458 \mathrm{e}-09 \backslash \mathrm{RMSF}=7.911 \mathrm{e}-05$

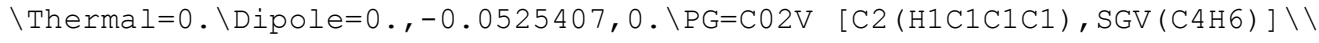
a 


\section{$\cdot \mathrm{CH}(\mathrm{Ph}) \mathrm{CH}_{3}$}

$1 \backslash 1 \backslash G I N C-S C 30 \backslash F O p t \backslash U B 3 L Y P \backslash 6-31 G(d) \backslash C 8 H 9(2) \backslash M L C 501 \backslash 25-M a y-2004 \backslash 0 \backslash \backslash \#$ B3L

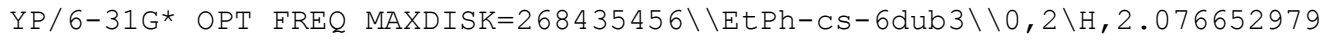
$1,-1.7071221701,0 . \backslash \mathrm{C}, 1.8330785143,-0.6472341403,0 . \backslash \mathrm{C}, 2.9648478287,0.33$ $29041964,0 . \backslash C, 0.4615044195,-0.296422334,0 . \backslash C,-2.3017509074,0.350177759$ $9,0 . \backslash C,-0.5414638247,-1.3098262805,0 . \backslash C, 0.01601966,1.0572057614,0 . \backslash C,-$ $1.3371627939,1.3659918739,0 . \backslash \mathrm{C},-1.8898621213,-0.9905058968,0 . \backslash \mathrm{H},-0.229$ $1124677,-2.3517513472,0 . \backslash \mathrm{H}, 0.7487305703,1.8588289809,0 . \backslash \mathrm{H},-1.649403422$ $1,2.4074639039,0 . \backslash \mathrm{H},-2.6317608755,-1.785156805,0 . \backslash \mathrm{H},-3.3593148857,0.59$ $85933381,0 . \backslash \mathrm{H}, 3.930913811,-0.179956881,0 . \backslash \mathrm{H}, 2.94101482,0.9926776704,0$. $8806011513 \backslash \mathrm{H}, 2.94101482,0.9926776704,-0.8806011513 \backslash \backslash$ Version=DEC $-\mathrm{AXP}-\mathrm{OS}$ $\mathrm{F} / 1-\mathrm{G} 03 \mathrm{RevB} .03 \backslash \mathrm{State}=2-\mathrm{A} " \backslash \mathrm{HF}=-310.2333536 \backslash \mathrm{S} 2=0.779917 \backslash \mathrm{S} 2-1=0 . \backslash \mathrm{S} 2 \mathrm{~A}=0.75$ $0613 \backslash \mathrm{RMSD}=8.432 \mathrm{e}-09 \backslash \mathrm{RMSF}=3.493 e-05 \backslash \mathrm{Dipole}=0.1484688,0.0260889,0 . \backslash \mathrm{PG}=\mathrm{CS}$ $[\mathrm{SG}(\mathrm{C} 8 \mathrm{H} 7), \mathrm{X}(\mathrm{H} 2)] \backslash \backslash \mathrm{Q}$

\section{$\cdot \mathrm{C}\left(\mathrm{CH}_{3}\right)_{2} \mathrm{Ph}$}

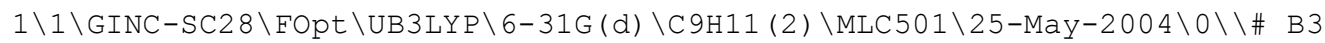

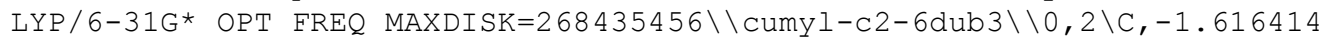
$3386,0 ., 0 . \backslash \mathrm{C},-0.1860957499,0 ., 0 . \backslash \mathrm{C}, 0.5630674826,1.2120912698,0 . \backslash \mathrm{C}, 0.56$ $30674826,-1.2120912698,0 . \backslash C, 1.9511243291,1.2069476466,0 . \backslash C, 1.951124329$ $1,-1.2069476466,0 . \backslash \mathrm{H}, 0.0402217434,2.1628990039,0 . \backslash \mathrm{H}, 0.0402217434,-2.16$ $28990039,0 . \backslash \mathrm{H}, 2.4890058288,2.1518597904,0 . \backslash \mathrm{H}, 2.4890058288,-2.151859790$ $4,0 . \backslash \mathrm{C},-2.382682254,1.2955409885,0 . \backslash \mathrm{C},-2.382682254,-1.2955409885,0 . \backslash \mathrm{H}$, $-3.4618855224,1.1182105536,0 . \backslash \mathrm{H},-3.4618855224,-1.1182105536,0 . \backslash \mathrm{H},-2.15$ $20630813,1.9147870606,0.8799660511 \backslash \mathrm{H},-2.1520630813,-1.9147870606,0.879$ $9660511 \backslash \mathrm{H},-2.1520630813,1.9147870606,-0.8799660511 \backslash \mathrm{H},-2.1520630813,-1$. $9147870606,-0.8799660511 \backslash \mathrm{C}, 2.6605839324,0 ., 0 . \backslash \mathrm{H}, 3.7470104688,0 ., 0 . \backslash \backslash \mathrm{Ve}$ rsion $=\mathrm{DEC}-\mathrm{AXP}-\mathrm{OSF} / 1-\mathrm{G} 03 \mathrm{RevB} .03 \backslash \mathrm{State}=2-\mathrm{B} 1 \backslash \mathrm{HF}=-349.5492998 \backslash \mathrm{S} 2=0.775843 \backslash$ $\mathrm{S} 2-1=0 . \backslash \mathrm{S} 2 \mathrm{~A}=0.750464 \backslash \mathrm{RMSD}=4.694 \mathrm{e}-09 \backslash \mathrm{RMSF}=4.133 \mathrm{e}-05 \backslash \mathrm{Dipole}=-0.2539911,0$ ., $0 . \backslash \mathrm{PG}=\mathrm{C} 02 \mathrm{~V}[\mathrm{C} 2(\mathrm{C} 1 \mathrm{C} 1 \mathrm{C} 1 \mathrm{H} 1), \mathrm{SGV}(\mathrm{C} 6 \mathrm{H} 6), \mathrm{X}(\mathrm{H} 4)] \backslash \backslash @$

\section{$\mathrm{CH}_{3} \mathrm{O} \bullet$}

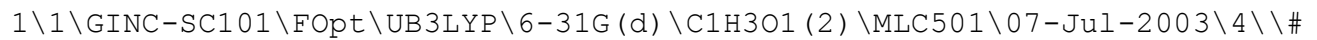
B3LYP/6-31G* OPT=TIGHT FREQ GUESS=ALTER MAXDISK=13107200 \CH3O_rad-c3v

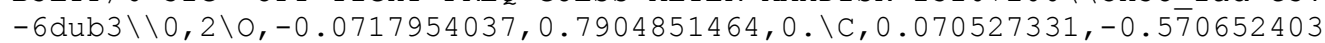
$1,0 . \backslash \mathrm{H},-0.9643070065,-0.9734523539,0 . \backslash \mathrm{H}, 0.557753125,-0.9632571993,0.90$ $88020881 \backslash \mathrm{H}, 0.557753125,-0.9632571993,-0.9088020881 \backslash \backslash \backslash 8,9 \backslash \backslash$ Version=DEC AXP-OSF / 1-G03RevB.03 \State $=2-A^{\prime} \backslash \mathrm{HF}=-115.0504626 \backslash \mathrm{S} 2=0.752916 \backslash \mathrm{S} 2-1=0 . \backslash \mathrm{S} 2$ $\mathrm{A}=0.750006 \backslash \mathrm{RMSD}=4.434 \mathrm{e}-09 \backslash \mathrm{RMSF}=5.514 \mathrm{e}-06 \backslash \mathrm{Dipole}=-0.0146059,-0.780437,0$ $. \backslash \mathrm{PG}=\mathrm{CS} \quad[\mathrm{SG}(\mathrm{C} 1 \mathrm{H} 1 \mathrm{O} 1), \mathrm{X}(\mathrm{H} 2)] \backslash \backslash$

\section{$\cdot \mathrm{OH}$}

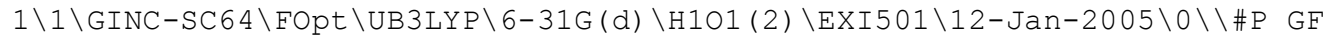
INPUT UB3LYP/6-31G* OPT=TIGHT FREQ MAXDISK=134217728 \\oh radical ub3IY

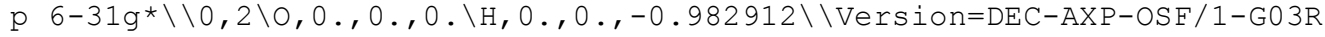

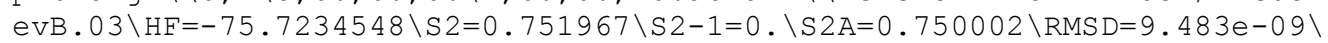
$\mathrm{RMSF}=2.457 \mathrm{e}-07 \backslash \mathrm{Dipole}=0 ., 0 ., 0.6914207 \backslash \mathrm{PG}=\mathrm{C} * \mathrm{~V}[\mathrm{C} *(\mathrm{H} 1 \mathrm{O} 1)] \backslash \backslash \mathrm{Q}$

\section{$\mathrm{H}-\mathrm{CH}_{3}$}

$1 \backslash 1 \backslash$ GINC-RSCQC2 $\backslash$ FOpt $\backslash R B 3 L Y P \backslash 6-31 G(d) \backslash C 1 H 4 \backslash M I C H E L L E \backslash 01-J u n-2001 \backslash 1 \backslash \backslash \# P \quad R$ B3LYP/6-31G* OPT=Z-MATRIX FREQ=NORAMAN MAXDISK=262144000 \METHANE TEST $\backslash \backslash 0,1 \backslash \mathrm{C} \backslash \mathrm{H}, 1, \mathrm{bl} \backslash \mathrm{H}, 1, \mathrm{bl}, 2,109.47122063 \backslash \mathrm{H}, 1, \mathrm{bl}, 2,109.47122063,3,120 ., 0 \backslash \mathrm{H}$, $1, \mathrm{bl}, 2,109.47122063,3,-120 ., 0 \backslash \backslash \mathrm{bl}=1.09326575 \backslash \backslash$ Version=IBM-RS 6000-G98Re $\mathrm{VA} .9 \backslash \mathrm{HF}=-40.5183892 \backslash \mathrm{RMSD}=1.077 \mathrm{e}-09 \backslash \mathrm{RMSF}=1.309 \mathrm{e}-08 \backslash \mathrm{Dipole}=0 ., 0 ., 0 . \backslash \mathrm{PG}=\mathrm{T}$ D $[\mathrm{O}(\mathrm{C} 1), 4 \mathrm{C} 3(\mathrm{H} 1)] \backslash \backslash \mathrm{e}$ 


\section{$\mathrm{H}-\mathrm{CH}_{2} \mathrm{CN}$}

$1 \backslash 1 \backslash G I N C-A C 16 \backslash F O p t \backslash R B 3 L Y P \backslash 6-31 G(d) \backslash C 2 H 3 N 1 \backslash E X I 501 \backslash 30-M a r-2007 \backslash 0 \backslash \backslash \# P$ gfi nput B3LYP/6-31G(d) INT (grid=ultrafine) opt freq=noraman maxdisk=13421 $77280 \backslash \backslash \mathrm{ppt} \backslash \backslash 0,1 \backslash \mathrm{C},-0.0012992462,0.0040237625,-0.0009187058 \backslash \mathrm{H}, 0.0069756$ $868,-0.0133335608,1.0935767355 \backslash \mathrm{H}, 1.0333592608,-0.0133335642,-0.3579488$ $435 \backslash \mathrm{H},-0.5095506754,-0.8964006992,-0.3603067338 \backslash \mathrm{C},-0.6854966132,1.2008$ $620522,-0.4847193037 \backslash \mathrm{N},-1.2286813285,2.1514843002,-0.8688088993 \backslash \backslash$ Versi on $=I A 64 \mathrm{~L}-\mathrm{G} 03$ RevD. $01 \backslash$ State $=1-\mathrm{A} ' \backslash H F=-132.7549285 \backslash \mathrm{RMS} D=4.773 \mathrm{e}-09 \backslash \mathrm{RMSF}=1.0$ $71 e-04 \backslash$ Thermal $=0 . \backslash$ Dipole $=0.7024936,-1.2290985,0.496738 \backslash \mathrm{PG}=\mathrm{CS} \quad[\mathrm{SG}(\mathrm{C} 2 \mathrm{H} 1 \mathrm{~N}$ 1), $\mathrm{X}(\mathrm{H} 2)] \backslash \backslash \mathrm{Q}$

\section{$\mathrm{H}-\mathrm{CH}_{2} \mathrm{OH}$}

$1 \backslash 1 \backslash G I N C-S C 123 \backslash F O p t \backslash R B 3 L Y P \backslash 6-31 G(d) \backslash C 1 H 4 O 1 \backslash M L C 501 \backslash 08-J u l-2003 \backslash 0 \backslash \backslash \#$ B3L

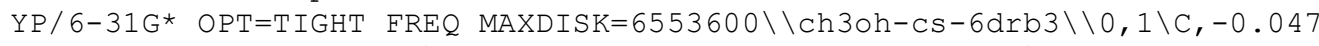
$3055047,0.660920544,0 . \backslash 0,-0.046396808,-0.7577091994,0 . \backslash \mathrm{H},-1.0948445488$ $, 0.9743407707,0 . \backslash \mathrm{H}, 0.8768642076,-1.0508634225,0 . \backslash \mathrm{H}, 0.436493917,1.08633$ $64915,0.8931833579 \backslash \mathrm{H}, 0.436493917,1.0863364915,-0.8931833579 \backslash \backslash$ Version=D $\mathrm{EC}-\mathrm{AXP}-\mathrm{OSF} / 1-\mathrm{G} 03 \mathrm{RevB} .03 \backslash \mathrm{State}=1-\mathrm{A}^{\prime} \backslash \mathrm{HF}=-115.7144073 \backslash \mathrm{RMSD}=1.496 \mathrm{e}-0 \mathrm{9} \backslash \mathrm{RMSF}$ $=4.832 \mathrm{e}-06 \backslash \mathrm{Dipole}=0.5654926,0.3529513,0 . \backslash \mathrm{PG}=\mathrm{CS} \quad[\mathrm{SG}(\mathrm{C} 1 \mathrm{H} 2 \mathrm{O} 1), \mathrm{X}(\mathrm{H} 2)] \backslash \backslash \mathrm{C}$

\section{$\mathrm{H}-\mathrm{CH}_{2} \mathbf{F}$}

$1 \backslash 1 \backslash G I N C-S C 32 \backslash F O p t \backslash R B 3 L Y P \backslash 6-31 G(d) \backslash C 1 H 3 F 1 \backslash R O O T \backslash 22-J u n-2001 \backslash 1 \backslash \backslash$ \# P B $L Y P$

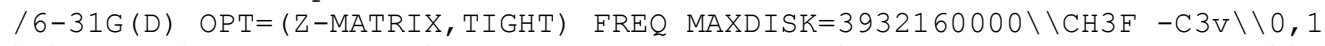
$\backslash \mathrm{C} \backslash \mathrm{F}, 1, \mathrm{Cf} \backslash \mathrm{H}, 1, \mathrm{ch}, 2, \mathrm{f} \mathrm{Ch} \backslash \mathrm{H}, 1, \mathrm{ch}, 2, \mathrm{fch}, 3,120 ., 0 \backslash \mathrm{H}, 1, \mathrm{ch}, 2, \mathrm{fch}, 3,-120 ., 0 \backslash \backslash \mathrm{C}$ $\mathrm{f}=1.38315398 \backslash \mathrm{ch}=1.09639705 \backslash \mathrm{f} \mathrm{ch}=109.61012707 \backslash \backslash$ Version=DEC-AXP-OSF/1-G98 RevA. 9 \HF=-139.733916 $\backslash \mathrm{RMSD}=7.738 \mathrm{e}-09 \backslash \mathrm{RMSF}=5.739 \mathrm{e}-07 \backslash \mathrm{Dipole}=0 ., 0 .,-0.67$ $44796 \backslash \mathrm{PG}=\mathrm{C} 03 \mathrm{~V} \quad[\mathrm{C} 3(\mathrm{C} 1 \mathrm{~F} 1), 3 \mathrm{SGV}(\mathrm{H} 1)] \backslash \backslash$

\section{$\mathrm{CH}_{3}-\mathrm{CH}_{2} \mathrm{CN}$}

$1 \backslash 1 \backslash G I N C-A C 1 \backslash F O p t \backslash R B 3 L Y P \backslash 6-31 G(d) \backslash C 3 H 5 N 1 \backslash E X I 501 \backslash 02-A p r-2007 \backslash 0 \backslash \backslash$ \# gfin put B3LYP/6-31G(d) INT (grid=ultrafine) opt freq=noraman maxdisk=134217 $7280 \backslash \backslash$ opt $\backslash \backslash 0,1 \backslash \mathrm{C},-0.0059599652,0.0103229625,-0.0614361274 \backslash \mathrm{C}, 0.01073949$ $,-0.0186013423,1.4778697843 \backslash \mathrm{H}, 1.0110723761,0.0212189515,-0.4639169434 \backslash$ $\mathrm{H},-0.5239123391,-0.865004887,-0.4639169434 \backslash \mathrm{H},-0.5249659516,0.909267700$ $4,-0.4080004238 \backslash \mathrm{H},-1.0125939979,-0.005429182,1.8723385858 \backslash \mathrm{C}, 0.69369120$ $62,-1.201508414,2.0127256558 \backslash \mathrm{H}, 0.5109988084,0.8742175349,1.8723385858 \backslash$ $\mathrm{N}, 1.2370789393,-2.1426835758,2.4206597603 \backslash \backslash$ Version=IA64L-G03RevD.01 \St ate $=1-\mathrm{A}^{\prime} \backslash \mathrm{HF}=-172.0686671 \backslash \mathrm{RMSD}=4.938 \mathrm{e}-09 \backslash \mathrm{RMSF}=2.520 \mathrm{e}-05 \backslash \mathrm{Thermal}=0 . \backslash \mathrm{Dipo}$ $l e=-0.7016957,1.2153726,-0.6298125 \backslash P G=C S \quad[S G(C 3 H 1 N 1), X(H 4)] \backslash \backslash @$

\section{$\mathrm{CH}_{3} \mathrm{CH}_{2} \mathrm{Ph}$}

$1 \backslash 1 \backslash G I N C-S C 95 \backslash F O p t \backslash R B 3 L Y P \backslash 6-31 G(d) \backslash C 8 H 10 \backslash E X I 501 \backslash 09-A p r-2005 \backslash 0 \backslash \backslash \# P$ GFIN PUT B3LYP/6-31G(D) OPT FREQ MAXDISK=134217728\\Et-Benzene $\backslash \backslash 0,1 \backslash \mathrm{H},-0.23$ $3900392,0.7968432572,-2.9432867998 \backslash C,-0.2303381036,0.8033110049,-1.856$ $1810065 \backslash \mathrm{C},-0.2343810639,0.7989760862,0.9273260884 \backslash \mathrm{C},-0.234380935,-0.40$ $36009571,-1.1555963365 \backslash C,-0.2280731409,2.0153711322,-1.1635762928 \backslash C,-0$ $.2303382373,2.0091555554,0.2324009485 \backslash \mathrm{C},-0.2355670878,-0.4255633581,0$. $2456993057 \backslash \mathrm{H},-0.2412016088,-1.3440246753,-1.703293985 \backslash \mathrm{H},-0.2286339879$, $2.9565771994,-1.7069823933 \backslash \mathrm{H},-0.2339006311,2.9473834185,0.7815542238 \backslash \mathrm{C}$ $,-0.1988542231,-1.7368636812,1.0027796846 \backslash \mathrm{H},-0.2412018371,0.80308522,2$ $.0156057341 \backslash \mathrm{C}, 1.233613206,-2.224977451,1.2845928797 \backslash \mathrm{H},-0.7346565065,-2$ $.505608687,0.4315000229 \backslash \mathrm{H},-0.7346567995,-1.6264927137,1.9541719643 \backslash \mathrm{H}, 1$ $.7869924931,-2.376264702,0.3509212704 \backslash \mathrm{H}, 1.2240845842,-3.1743111998,1.8$ $326915593 \backslash \mathrm{H}, 1.7869921996,-1.4920371047,1.882446777 \backslash \backslash$ Version=Al $64 \mathrm{~T}-\mathrm{G} 03 \mathrm{R}$ evC.02\State $=1-A \backslash H F=-310.8802504 \backslash \mathrm{RMSD}=3.633 e-09 \backslash \mathrm{RMSF}=1.696 \mathrm{e}-05 \backslash \mathrm{Dipole}=$ $0.0171231,-0.0942059,0.0543899 \backslash \mathrm{PG}=\mathrm{C} 01 \quad[\mathrm{X}(\mathrm{C} 8 \mathrm{H} 10)] \backslash \backslash @$ 


\section{$\mathrm{CH}_{3} \mathrm{CH}_{2} \mathrm{~F}$}

$1 \backslash 1 \backslash G I N C-A C 9 \backslash F O p t \backslash R B 3 L Y P \backslash 6-31 G(d) \backslash C 2 H 5 F 1 \backslash E X I 501 \backslash 02-A p r-2007 \backslash 0 \backslash \backslash \# P$ gfin put B3LYP/6-31G (d) INT (grid=ultrafine) opt freq=noraman maxdisk=134217 $7280 \backslash \backslash \mathrm{ppt} \backslash \backslash 0,1 \backslash \mathrm{C},-0.0019457842,0.0033701971,-0.0383960874 \backslash \mathrm{C},-0.0073010$ $981,0.0126458729,1.4783551467 \backslash \mathrm{H}, 1.0246032765,0.0011278765,-0.418774514$ $6 \backslash \mathrm{H},-0.5132784079,-0.8867685279,-0.4187745146 \backslash \mathrm{H},-0.5142555168,0.890716$ $6832,-0.4277897465 \backslash \mathrm{H},-1.0310931261,0.0061665786,1.8736216226 \backslash \mathrm{F}, 0.64667$ $29937,-1.120070481,1.9584698075 \backslash \mathrm{H}, 0.5102061493,0.8960361302,1.87362162$ $26 \backslash \backslash$ Version=IA64L-G03RevD.01 \State $=1-A^{\prime} \backslash \mathrm{HF}=-179.0564219 \backslash \mathrm{RMSD}=6.423 \mathrm{e}-09$ $\backslash \mathrm{RMSF}=1.039 \mathrm{e}-04 \backslash \mathrm{Thermal}=0 . \backslash \mathrm{Dipole}=-0.3022584,0.5235269,-0.3012431 \backslash \mathrm{PG}=\mathrm{C}$ $S \quad[S G(C 2 H 1 F 1), X(H 4)] \backslash \backslash @$

\section{$\mathrm{CH}_{3} \mathrm{CH}_{2} \mathrm{OH}$}

$1 \backslash 1 \backslash G I N C-A C 9 \backslash F O p t \backslash R B 3 L Y P \backslash 6-31 G(d) \backslash C 2 H 601 \backslash E X I 501 \backslash 02-A p r-2007 \backslash 0 \backslash \backslash \# P$ gfin put B3LYP/6-31G(d) INT (grid=ultrafine) opt freq=noraman maxdisk=134217 $7280 \backslash \backslash$ opt $\backslash \backslash 0,1 \backslash \mathrm{C},-0.0006062236,0.0010500101,-0.0307568131 \backslash \mathrm{C},-0.0026017$ $523,0.0045063672,1.4886360801 \backslash \mathrm{H}, 1.0263560636,-0.0038781106,-0.41066633$ $89 \backslash \mathrm{H},-0.5098194895,-0.8907894797,-0.4106663389 \backslash \mathrm{H},-0.5128201384,0.88823$ $05349,-0.420039437 \backslash \mathrm{H},-1.0399148855,0.0264531962,1.8621128508 \backslash 0,0.67498$ $81633,-1.1691137933,1.9286779957 \backslash \mathrm{H}, 0.4970483028,0.9138193067,1.8621128$ $508 \backslash \mathrm{H}, 0.6739430923,-1.1673036772,2.8979224905 \backslash \backslash$ Version=IA64L-G03RevD.0 $1 \backslash$ State $=1-\mathrm{A} \backslash \backslash \mathrm{HF}=-155.0337991 \backslash \mathrm{RMSD}=6.372 \mathrm{e}-09 \backslash \mathrm{RMSF}=1.024 \mathrm{e}-04 \backslash$ Thermal $=0 . \backslash$ Dipole $=-0.2356189,0.4081039,0.3948316 \backslash P G=C S \quad[S G(C 2 H 2 O 1), X(H 4)] \backslash \backslash @$

\section{$\mathrm{CH}_{3} \mathrm{CH}_{3}$}

$1 \backslash 1 \backslash G I N C-S C 72 \backslash$ Freq $\backslash R B 3 L Y P \backslash 6-31 G(d) \backslash C 2 H 6 \backslash E X I 501 \backslash 20-J a n-2005 \backslash 1 \backslash \backslash \# P$ GFINP UT B3LYP/6-31G* SCF=TIGHT FREQ MAXDISK=134217728\\c2h6 b3-lyp freq 6-3 $1 \mathrm{~g} \star \backslash \backslash 0,1 \backslash \mathrm{C} \backslash \mathrm{C}, 1, \mathrm{cc} 2 \backslash \mathrm{H}, 2, \mathrm{hc} 3,1, \mathrm{hcc} 3 \backslash \mathrm{H}, 2, \mathrm{hc} 4,3, \mathrm{hch} 4,1, \mathrm{dih} 4,0 \backslash \mathrm{H}, 2, \mathrm{hc} 5,4$, hc h5, 3, dih5, $0 \backslash \mathrm{H}, 1, \mathrm{hc} 6,2$, hcc $6,3, \operatorname{dih} 6,0 \backslash \mathrm{H}, 1, \mathrm{hc7}, 2, \mathrm{hcc} 7,3, \operatorname{dih} 7,0 \backslash \mathrm{H}, 1, \mathrm{hc} 8,2$, $\mathrm{hcc} 8,3, \operatorname{dih} 8,0 \backslash \backslash \mathrm{cc} 2=1.530542 \backslash \mathrm{hc} 3=1.096162 \backslash \mathrm{hcc} 3=111.35 \backslash \mathrm{hc} 4=1.096162 \backslash \mathrm{hch} 4$ $=107.529 \backslash \mathrm{dih} 4=-122.235 \backslash \mathrm{hc} 5=1.096162 \backslash \mathrm{hch} 5=107.529 \backslash \mathrm{dih} 5=115.53 \backslash \mathrm{hc} 6=1.096$ $162 \backslash \mathrm{hcc} 6=111.35 \backslash \mathrm{dih} 6=180 . \backslash \mathrm{hc7}=1.096162 \backslash \mathrm{hcc} 7=111.35 \backslash \mathrm{dih} 7=-60 . \backslash \mathrm{hc} 8=1.096$ $162 \backslash$ hcc $8=111.35 \backslash$ dih $8=60 . \backslash \backslash$ Version=DEC-AXP-OSF $/ 1-G 03 R e v B .03 \backslash$ State $=1-A \backslash H$ $\mathrm{F}=-79.8304178 \backslash \mathrm{RMSD}=6.267 \mathrm{e}-09 \backslash \mathrm{RMSF}=4.351 \mathrm{e}-06 \backslash \mathrm{Dipole}=0.0000011,-0.000001$ $2,0.0000001 \backslash$ DipoleDeriv $=0.1179333,-0.0000159,-0.0000136,-0.0000154,0.1$ $179506,0.0000246,-0.0000197,0.0000347,0.0840561,0.1179319,-0.0000165,-$ $0.0000149,-0.000017,0.1179518,0.0000254,-0.0000209,0.0000352,0.0840569$ $,-0.1413675,-0.000014,-0.0960899,0.0000075,0.0627172,-0.0000109,-0.070$ $2022,-0.0000215,-0.0280191,0.0117388,0.0883908,0.048051,0.0883908,-0.0$ $903236,-0.0832254,0.0351038,-0.0608009,-0.0280191,0.0116962,-0.0883604$ $, 0.0480533,-0.0883819,-0.0903454,0.0832116,0.0351195,0.060787,-0.02801$ $96,-0.1413656,-0.0000132,-0.0960897,0.0000071,0.0627173,-0.0000101,-0$. $070202,-0.0000206,-0.0280186,0.0116941,-0.0883616,0.0480534,-0.0883819$ $,-0.090342,0.0832108,0.0351184,0.0607863,-0.0280185,0.0117388,0.088390$ $8,0.0480505,0.0883907,-0.0903259,-0.0832259,0.0351032,-0.0608003,-0.02$ $80181 \backslash$ Polar $=22.4151706,0.0001301,22.4150143,0.0000451,-0.0000938,23.93$ $4685 \backslash P G=C 01 \quad[X(C 2 H 6)] \backslash N I m a g=0 \backslash \backslash @$

\section{$\mathrm{CH}_{3} \mathrm{CH}\left(\mathrm{CH}_{3}\right) \mathrm{Ph}$}

$1 \backslash 1 \backslash G I N C-S C 116 \backslash F O p t \backslash R B 3 L Y P \backslash 6-31 G(d) \backslash C 8 H 10 \backslash E X I 501 \backslash 02-A p r-2005 \backslash 0 \backslash \backslash \# P$ GFI NPUT B3LYP/6-31G(D) OPT FREQ MAXDISK=134217728 MAXDISK=134217728 \\Et-B enzene $\backslash \backslash 0,1 \backslash C,-1.9550533926,-0.175555431,-1.2711663096 \backslash C,-1.99489403,-$ $0.391830921,0.1074633098 \backslash C,-0.8111522177,-0.4244684066,0.8457664203 \backslash C$, $0.4323908384,-0.2420109736,0.2256228116 \backslash \mathrm{C}, 0.4568522382,-0.0274597572$, $1.1590910756 \backslash C,-0.723528312,0.0062456715,-1.902870573 \backslash C, 1.7122853509,-$ $0.2386753228,1.0356140448 \backslash \mathrm{H}, 1.6079423627,-0.9203737835,1.8893428765 \backslash \mathrm{C}$, $2.0904517917,1.1614466288,1.5517128073 \backslash \mathrm{H},-0.8508277059,-0.598599829,1$. $9193225743 \backslash \mathrm{H}, 1.4130110341,0.1102371299,-1.6603159853 \backslash \mathrm{H},-2.9482067102,-$ $0.5401682081,0.6085302262 \backslash \mathrm{H},-0.6806109241,0.1697887361,-2.9767827541 \backslash \mathrm{H}$ $,-2.8753940816,-0.1533940371,-1.8487484216 \backslash \mathrm{H}, 2.5347775634,-0.630362643$ $4,0.4236272983 \backslash \mathrm{H}, 1.3016904361,1.5687814609,2.194178615 \backslash \mathrm{H}, 2.233991779,1$ 
$.8603210864,0.719954068 \backslash \mathrm{H}, 3.019512645,1.1276211595,2.132582889 \backslash \backslash$ Versio $\mathrm{n}=\mathrm{DEC}-\mathrm{AXP}-\mathrm{OSF} / 1-\mathrm{G} 03 \mathrm{RevB} .03 \backslash \mathrm{State}=1-\mathrm{A} \backslash \mathrm{HF}=-310.8802504 \backslash \mathrm{RMSD}=2.757 \mathrm{e}-09 \backslash \mathrm{RM}$ $\mathrm{SF}=1.402 \mathrm{e}-05 \backslash \mathrm{Dipole}=0.091025,0.0142741,0.0603704 \backslash \mathrm{PG}=\mathrm{C} 01 \quad[\mathrm{X}(\mathrm{C} 8 \mathrm{H} 10)] \backslash \backslash @$

\section{$\mathrm{CH}_{3} \mathrm{C}\left(\mathrm{CH}_{3}\right)_{2} \mathrm{Ph}$}

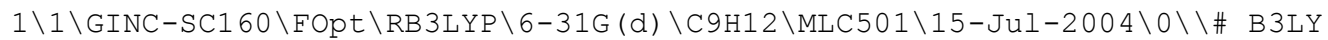

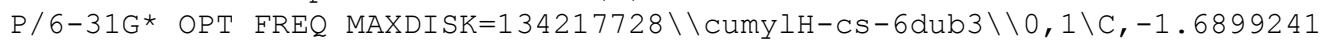
$576,-0.1717677053,0 . \backslash \mathrm{C},-0.166110256,-0.1721242299,0 . \backslash \mathrm{C}, 0.5651701726,1$. $025588545,0 . \backslash C, 1.959755901,1.0169364848,0 . \backslash C, 0.5469439405,-1.377640064$ $8,0 . \backslash \mathrm{C}, 1.9433393106,-1.393118293,0 . \backslash \mathrm{C}, 2.6557562567,-0.1940404185,0 . \backslash \mathrm{H}$, $0.0402648699,1.9781154329,0 . \backslash \mathrm{H}, 0.0000587161,-2.3183655685,0 . \backslash \mathrm{H}, 2.50448$ $70428,1.9578251307,0 . \backslash \mathrm{H}, 2.4725022991,-2.3427864574,0 . \backslash \mathrm{H}, 3.7425448432,-$ $0.2012622131,0 . \backslash \mathrm{H},-2.0123581199,-1.2220929864,0 . \backslash \mathrm{C},-2.2637087336,0.485$ $062341,1.2695572918 \backslash \mathrm{C},-2.2637087336,0.485062341,-1.2695572918 \backslash \mathrm{H},-1.992$ $4183132,1.5459811224,1.3260966421 \backslash \mathrm{H},-1.9924183132,1.5459811224,-1.3260$ $966421 \backslash \mathrm{H},-1.885411406,-0.0043887136,2.1735361138 \backslash \mathrm{H},-1.885411406,-0.004$ $3887136,-2.1735361138 \backslash \mathrm{H},-3.3584612085,0.4208139215,1.2778278313 \backslash \mathrm{H},-3.3$ $584612085,0.4208139215,-1.2778278313 \backslash \backslash$ Version=DEC-AXP-OSF/1-G03RevB.03 $\backslash$ State $=1-A^{\prime} \backslash H F=-350.193313 \backslash R M S D=5.904 e-09 \backslash R M S F=3.037 e-05 \backslash D i p o l e=-0.105$ $8048,-0.0014748,0 . \backslash P G=C S \quad[S G(C 7 H 6), X(C 2 H 6)] \backslash \backslash @$

\section{$\mathrm{FCH}_{2} \mathrm{CN}$}

$1 \backslash 1 \backslash G I N C-A C 16 \backslash F O p t \backslash R B 3 L Y P \backslash 6-31 G(d) \backslash C 2 H 2 F 1 N 1 \backslash E X I 501 \backslash 30-M a r-2007 \backslash 0 \backslash \backslash \# P \quad g$ finput B3LYP/6-31G(d) INT (grid=ultrafine) opt freq=noraman maxdisk=134 $2177280 \backslash \backslash \mathrm{opt} \backslash \backslash 0,1 \backslash \mathrm{C}, 0.0205683986,0.0322046687,0.0145794899 \backslash \mathrm{H}, 0.0249904$ $82,0.0299863409,1.1105886615 \backslash \mathrm{H}, 1.05581711,0.0312839858,-0.345420934 \backslash \mathrm{C}$, $-0.6673049383,-1.1724551427,-0.4755546438 \backslash \mathrm{F},-0.619406546,1.1684311584$, $-0.4373184746 \backslash \mathrm{N},-1.1978112196,-2.1265845916,-0.8663423413 \backslash \backslash$ Version $=$ IA 6 4L-G03RevD. 01 \State $=1-A \backslash H F=-231.9668007 \backslash R M S D=8.232 e-09 \backslash R M S F=2.219 e-04 \backslash$ Thermal=0. $\backslash$ Dipole $=0.8971186,0.5454858,0.6440098 \backslash \mathrm{PG}=\mathrm{C} 01 \quad[\mathrm{X}(\mathrm{C} 2 \mathrm{H} 2 \mathrm{~F} 1 \mathrm{~N} 1)] \backslash \backslash$ a

\section{$\mathrm{FCH}_{2} \mathrm{~F}$}

$1 \backslash 1 \backslash G I N C-A C 7 \backslash F O p t \backslash R B 3 L Y P \backslash 6-31 G(d) \backslash C 1 H 2 F 2 \backslash E X I 501 \backslash 30-M a r-2007 \backslash 0 \backslash \backslash \# P$ gfin put B3LYP/6-31G(d) INT (grid=ultrafine) opt freq=noraman maxdisk=134217 $7280 \backslash \backslash \mathrm{opt} \backslash \backslash 0,1 \backslash \mathrm{C}, 0.0124702627,0 ., 0.0088178073 \backslash \mathrm{H},-0.0135869694,0 ., 1.104$ $3010126 \backslash \mathrm{H}, 1.0366159895,0 .,-0.3809102551 \backslash \mathrm{F},-0.6313581309,-1.1093052856$, $-0.4464376157 \backslash F,-0.6313581309,1.1093052856,-0.4464376157 \backslash \backslash$ Version=IA 64 $\mathrm{L}-\mathrm{G} 03$ RevD $.01 \backslash$ State $=1-\mathrm{A} 1 \backslash \mathrm{HF}=-238.9732009 \backslash \mathrm{RMSD}=2.203 \mathrm{e}-09 \backslash \mathrm{RMSF}=6.642 \mathrm{e}-05 \backslash$ Thermal $=0 . \backslash$ Dipole $=0.5512984,0 ., 0.3898269 \backslash \mathrm{PG}=\mathrm{C} 02 \mathrm{~V}$ [C2 (C1), SGV (F2), SGV' ( H2) $] \backslash \backslash @$

\section{$\mathrm{HOCH}_{2} \mathrm{~F}$}

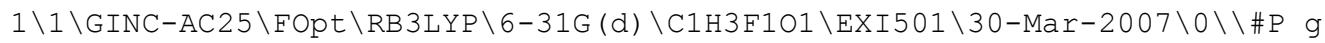
finput B3LYP/6-31G(d) INT (grid=ultrafine) opt freq=noraman maxdisk=134 $2177280 \backslash \backslash$ opt $\backslash \backslash 0,1 \backslash \mathrm{C},-0.0010409755,-0.0156677076,-0.0108673569 \backslash \mathrm{H},-0.015$ $1101164,-0.0395756805,1.0819615589 \backslash \mathrm{H}, 1.0312467969,0.0206583129,-0.3876$ $154639 \backslash 0,-0.6861562106,-1.1372790606,-0.4390307662 \backslash \mathrm{F},-0.6205814079,1.1$ $507835137,-0.4350927394 \backslash \mathrm{H},-0.6285397111,-1.1791693518,-1.4070218991 \backslash \backslash \mathrm{V}$ ersion=IA64L-G03RevD.01 \State $=1-A \backslash H F=-214.9554667 \backslash$ RMSD $=4.106 \mathrm{e}-09 \backslash \mathrm{RMSF}=$ $7.705 e-05 \backslash$ Thermal $=0 . \backslash$ Dipole $=0.5332521,-0.2312289,-0.2940706 \backslash P G=C 01 \quad[X($ $\mathrm{C} 1 \mathrm{H} 3 \mathrm{~F} 101)] \backslash \backslash \mathrm{Q}$

\section{MeF}

$1 \backslash 1 \backslash G I N C-S C 125 \backslash F r e q \backslash R B 3 L Y P \backslash 6-31 G(d) \backslash C 1 H 3 F 1 \backslash E X I 501 \backslash 20-J a n-2005 \backslash 1 \backslash \backslash \# P \quad G F$ INPUT B3LYP/6-31G* SCF=TIGHT FREQ MAXDISK=134217728\\b31yp 6-31g* for ch3 $\backslash \backslash \backslash 0,1 \backslash \mathrm{C} \backslash \mathrm{F}, 1, \mathrm{fc} 2 \backslash \mathrm{H}, 1, \mathrm{hc} 3,2, \operatorname{hcf} 3 \backslash \mathrm{H}, 1, \mathrm{hc} 4,3, \mathrm{hch} 4,2, \operatorname{dih} 4,0 \backslash \mathrm{H}, 1, \mathrm{hc} 5,4, \mathrm{~h}$ ch5, 3, dih5, $0 \backslash \backslash \mathrm{fc} 2=1.383154 \backslash \mathrm{hc} 3=1.096397 \backslash \mathrm{hcf} 3=109.61 \backslash \mathrm{hc} 4=1.096396 \backslash \mathrm{hch} 4=$

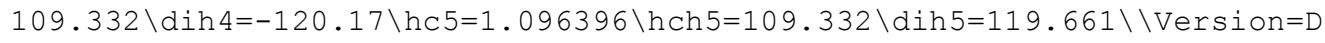
$\mathrm{EC}-\mathrm{AXP}-\mathrm{OSF} / 1-\mathrm{G} 03 \mathrm{RevB} .03 \backslash \mathrm{Sta}$ te $=1-\mathrm{A} \backslash \mathrm{HF}=-139.7339159 \backslash \mathrm{RMSD}=4.279 \mathrm{e}-09 \backslash \mathrm{RMSF}=$ $2.181 e-06 \backslash$ Dipole $=0.0000031,-0.0000018,-0.6744813 \backslash$ DipoleDeriv $=0.4243356$ $, 0.0000532,0.0000131,0.0000163,0.4243462,0.000022,-0.0000131,0.000085$, 
$0.958972,-0.2534428,-0.0000111,0.0000069,-0.0000109,-0.2534674,0.00000$ $45,0.0000029,-0.0000401,-0.8576188,-0.1602921,0.0000059,0.0771548,-0.0$ $000033,0.0463906,-0.0000005,0.0446842,-0.0000103,-0.0337785,-0.0053007$ $,-0.0895136,-0.0385888,-0.0894909,-0.1086532,-0.0668435,-0.0223289,-0$. $0387162,-0.0337871,-0.0053,0.0894656,-0.0385859,0.0894888,-0.1086162,0$ $.0668174,-0.0223451,0.0386815,-0.0337877 \backslash$ Polar $=12.8414473,0.0000468,12$ $.8414163,0.0000327,0.0001686,12.724623 \backslash \mathrm{PG}=\mathrm{C} 01 \quad[\mathrm{X}(\mathrm{C} 1 \mathrm{H} 3 \mathrm{~F} 1)] \backslash \mathrm{NImag}=0 \backslash \backslash$

\section{$\mathrm{F}-\mathrm{CH}_{2} \mathrm{Ph}$}

$1 \backslash 1 \backslash G I N C-S C 64 \backslash F O p t \backslash R B 3 L Y P \backslash 6-31 G(d) \backslash C 7 H 7 F 1 \backslash E X I 501 \backslash 24-A p r-2005 \backslash 0 \backslash \backslash \# P \quad$ GFI

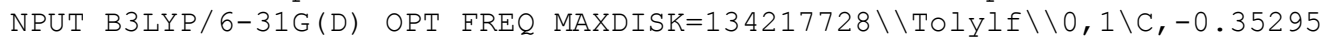
$6208,-0.0149093559,-0.9974543921 \backslash C,-0.341786443,-0.0415843639,0.399858$ $6096 \backslash C, 0.8837534814,-0.0782820042,1.0750072734 \backslash C, 2.0826191008,-0.09657$ $72136,0.3625807358 \backslash \mathrm{C}, 2.0673107129,-0.0697567899,-1.0337609231 \backslash \mathrm{C}, 0.8473$ $475345,-0.0259609441,-1.7099655183 \backslash C,-1.6338418746,-0.0705548639,1.180$ $6884614 \backslash \mathrm{F},-2.7040248188,0.3509932368,0.3992450859 \backslash \mathrm{H}, 0.8280912897,0.000$ $4661203,-2.7964011699 \backslash \mathrm{H},-1.3034105858,0.0250590333,-1.5188907598 \backslash \mathrm{H}, 3.0$ $00946916,-0.0784976018,-1.5897556294 \backslash \mathrm{H}, 3.0281974004,-0.1236240567,0.89$ $77140308 \backslash \mathrm{H}, 0.9027291824,-0.0896846375,2.1633371295 \backslash \mathrm{H},-1.860603611,-1.0$ $883825801,1.5274181261 \backslash \mathrm{H},-1.5744050468,0.5814778035,2.0616470188 \backslash \backslash$ Vers ion=Al $64 \mathrm{~T}-\mathrm{G} 03 \mathrm{RevC} .02 \backslash$ State $=1-\mathrm{A} \backslash \mathrm{HF}=-370.7906943 \backslash \mathrm{RMSD}=2.566 \mathrm{e}-09 \backslash \mathrm{RMSF}=4.8$ $90 e-06 \backslash \mathrm{Dipole}=0.4827353,-0.1930635,0.2941916 \backslash \mathrm{PG}=\mathrm{C} 01 \quad[\mathrm{X}(\mathrm{C} 7 \mathrm{H} 7 \mathrm{~F} 1)] \backslash \backslash \mathrm{d}$

\section{$\mathrm{F}-\mathrm{CH}\left(\mathrm{CH}_{3}\right) \mathrm{Ph}$}

$1 \backslash 1 \backslash G I N C-S C 121 \backslash F O p t \backslash R B 3 L Y P \backslash 6-31 G(d) \backslash C 8 H 9 F 1 \backslash E X I 501 \backslash 09-A p r-2005 \backslash 0 \backslash \backslash \# P \quad G F$

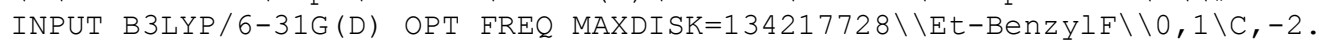
$1997715127,-0.2004541044,0.0631184737 \backslash \mathrm{C},-2.1853229814,-0.1401869644,1$. $4575546375 \backslash C,-0.9648294927,-0.0846874571,2.1338570505 \backslash C, 0.2317129646,-$ $0.0884836828,1.416845782 \backslash \mathrm{C}, 0.2224162322,-0.1404461196,0.0180346432 \backslash \mathrm{C},-$ $1.0025610135,-0.1997805798,-0.6541845128 \backslash \mathrm{C}, 1.525295489,-0.0927111747,-$ $0.7525767558 \backslash \mathrm{C}, 2.0635342986,1.3222475531,-0.9384796561 \backslash \mathrm{H}, 2.2769717638$, $-0.7140464402,-0.2472358511 \backslash \mathrm{H}, 1.1798904915,-0.0556032961,1.9504275137 \backslash$ $\mathrm{H},-1.0099688425,-0.2590685913,-1.7374394764 \backslash \mathrm{H},-0.9435972636,-0.0464196$ $758,3.219842943 \backslash \mathrm{H},-3.1459255747,-0.2517344726,-0.4694522106 \backslash \mathrm{H},-3.11829$ $81251,-0.1424165747,2.0147937127 \backslash \mathrm{F}, 1.3368359654,-0.6453334896,-2.02654$ $86101 \backslash \mathrm{H}, 2.2735962817,1.7855528676,0.0311158304 \backslash \mathrm{H}, 1.3256130043,1.938463$ $6768,-1.4622698076 \backslash \mathrm{H}, 2.9873506703,1.3002890905,-1.5258631368 \backslash \backslash$ Version= Al64T-G03RevC.02 $\backslash$ State $=1-A \backslash H F=-410.112402 \backslash$ RMSD $=9.876 \mathrm{e}-09 \backslash \mathrm{RMSF}=4.176 \mathrm{e}-0$ $6 \backslash$ Dipole $=0.0583468,0.3091603,0.5230204 \backslash P G=C 01 \quad[X(C 8 H 9 F 1)] \backslash \backslash @$

\section{$\mathrm{F}-\mathrm{C}\left(\mathrm{CH}_{3}\right)_{2} \mathbf{P h}$}

$1 \backslash 1 \backslash G I N C-S C 2 \backslash F O p t \backslash R B 3 L Y P \backslash 6-31 G(d) \backslash C 9 H 11 F 1 \backslash E X I 501 \backslash 19-A p r-2005 \backslash 0 \backslash \backslash \# P$ GFI NPUT B3LYP/6-31G(D) OPT FREQ MAXDISK=134217728\\CumyI $F \backslash \backslash 0,1 \backslash C, 2.88228$ $0297,-0.0244711185,0 . \backslash C, 2.1882826554,1.1872148711,0 . \backslash C, 0.7938467583,1$. $1956988269,0 . \backslash C, 0.0698989409,-0.0050562537,0 . \backslash C, 0.7727301943,-1.214656$ $192,0 . \backslash C, 2.1690430475,-1.223006466,0 . \backslash C,-1.4553798655,0.0096990777,0 . \backslash$ $C,-2.0228784388,0.6426802749,-1.272237705 \backslash C,-2.0228784388,0.6426802749$ $, 1.272237705 \backslash \mathrm{H}, 0.2695220331,2.1483611544,0 . \backslash \mathrm{H}, 0.2206714415,-2.14714288$ $45,0 . \backslash \mathrm{H}, 2.7321161998,2.1283132697,0 . \backslash \mathrm{H}, 2.6990304692,-2.1721067261,0 . \backslash \mathrm{H}$ $, 3.968970323,-0.0325289493,0 . \backslash \mathrm{F},-1.9051088729,-1.3298325407,0 . \backslash \mathrm{H},-1.72$ $59440977,1.69261363,1.3575810419 \backslash \mathrm{H},-1.7259440977,1.69261363,-1.3575810$ $419 \backslash \mathrm{H},-1.6544759779,0.1077592192,2.1527151544 \backslash \mathrm{H},-1.6544759779,0.107759$ $2192,-2.1527151544 \backslash \mathrm{H},-3.1165806805,0.5890757662,1.2597888852 \backslash \mathrm{H},-3.1165$ $806805,0.5890757662,-1.2597888852 \backslash \backslash$ Version=Al $64 \mathrm{~T}-\mathrm{G} 03$ RevC.02 $\backslash$ State=1-A ${ }^{\prime}$ $\backslash \mathrm{HF}=-449.4314132 \backslash \mathrm{RMSD}=9.882 \mathrm{e}-09 \backslash \mathrm{RMSF}=4.404 \mathrm{e}-06 \backslash \mathrm{Dipole}=0.1396441,0.5657$ $134,0 . \backslash \mathrm{PG}=\mathrm{CS} \quad[\mathrm{SG}(\mathrm{C} 7 \mathrm{H} 5 \mathrm{~F} 1), \mathrm{X}(\mathrm{C} 2 \mathrm{H} 6)] \backslash \backslash @$

\section{$\mathrm{HOCH}_{2} \mathrm{CN}$}

$1 \backslash 1 \backslash G I N C-A C 6 \backslash F O p t \backslash R B 3 L Y P \backslash 6-31 G(d) \backslash C 2 H 3 N 101 \backslash E X I 501 \backslash 03-A p r-2007 \backslash 0 \backslash \backslash \# P \quad g f$ input B3LYP/6-31G(d) INT (grid=ultrafine) opt freq=noraman maxdisk=1342 $177280 \backslash \backslash$ opt $\backslash \backslash 0,1 \backslash \mathrm{C},-0.023466606,0.004950111,0.000154213 \backslash \mathrm{H}, 0.011766849$, 
$-0.035876881,1.099280239 \backslash \mathrm{H}, 1.009047171,0.028204368,-0.360702176 \backslash \mathrm{C},-0.6$ $7432002,-1.229401698,-0.489213506 \backslash 0,-0.643196145,1.179729757,-0.482357$ $717 \backslash \mathrm{H},-1.552827358,1.203586319,-0.145483398 \backslash \mathrm{N},-1.22601721,-2.178344676$ ,$-0.866010989 \backslash \backslash$ Version=IA64L-G03RevD.01 \State $=1-A \backslash H F=-207.9510253 \backslash$ RMSD $=5.446 e-09 \backslash \mathrm{RMSF}=8.167 \mathrm{e}-05 \backslash \mathrm{Thermal}=0 . \backslash \mathrm{Dipole}=-0.9288242,0.4410303,0.541$ $5698 \backslash \mathrm{PG}=\mathrm{C} 01 \quad[\mathrm{X}(\mathrm{C} 2 \mathrm{H} 3 \mathrm{~N} 1 \mathrm{O} 1)] \backslash \backslash \mathrm{Q}$

\section{$\mathrm{HOCH}_{2} \mathrm{OH}$}

$1 \backslash 1 \backslash G I N C-A C 15 \backslash F O p t \backslash R B 3 L Y P \backslash 6-31 G(d) \backslash C 1 H 402 \backslash E X I 501 \backslash 01-A p r-2007 \backslash 0 \backslash \backslash \# P$ gfi nput B3LYP/6-31G(d) INT (grid=ultrafine) opt freq=noraman maxdisk=13421 $77280 \backslash \backslash \mathrm{ppt} \backslash \backslash 0,1 \backslash \mathrm{C},-0.0504592938,0.0193970689,0.0405071511 \backslash 0,-0.0268173$ $331,-0.0437268469,1.4461265279 \backslash \mathrm{H}, 0.9968836742,0.1648707894,-0.25334840$ $45 \backslash \mathrm{H},-0.9384343996,-0.2118821756,1.7365780715 \backslash \mathrm{H},-0.4319072512,-0.90467$ $17361,-0.4122504751 \backslash 0,-0.8900805756,1.0366167726,-0.4493217969 \backslash \mathrm{H},-0.55$ $27125919,1.8761546856,-0.0955718901 \backslash \backslash$ Version=IA64L-G03RevD .01\State=1$\mathrm{A} \backslash \mathrm{HF}=-190.9364376 \backslash \mathrm{RMSD}=3.753 \mathrm{e}-09 \backslash \mathrm{RMSF}=3.872 \mathrm{e}-05 \backslash$ Therma $\mathrm{l}=0 . \backslash \mathrm{Dipol} \mathrm{e}=-0.0$ $726001,0.0848727,0.0809846 \backslash P G=C 01 \quad[X(\mathrm{C} 1 \mathrm{H} 4 \mathrm{O} 2)] \backslash \backslash @$

\section{$\mathrm{MeOH}$}

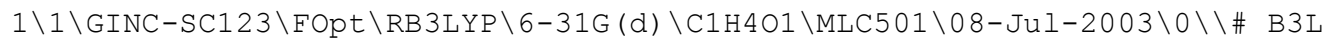

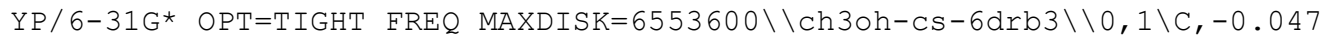
$3055047,0.660920544,0 . \backslash 0,-0.046396808,-0.7577091994,0 . \backslash \mathrm{H},-1.0948445488$ $, 0.9743407707,0 . \backslash \mathrm{H}, 0.8768642076,-1.0508634225,0 . \backslash \mathrm{H}, 0.436493917,1.08633$ $64915,0.8931833579 \backslash \mathrm{H}, 0.436493917,1.0863364915,-0.8931833579 \backslash \backslash$ Version=D EC-AXP-OSF/1-G03RevB.03 \State $=1-A^{\prime} \backslash H F=-115.7144073 \backslash R M S D=1.496 e-09 \backslash$ RMSF $=4.832 e-06 \backslash \mathrm{Dipole}=0.5654926,0.3529513,0 . \backslash \mathrm{PG}=\mathrm{CS} \quad[\mathrm{SG}(\mathrm{C} 1 \mathrm{H} 2 \mathrm{O} 1), \mathrm{X}(\mathrm{H} 2)] \backslash \backslash \mathrm{e}$

\section{$\mathrm{HO}-\mathrm{CH}_{2} \mathrm{Ph}$}

$1 \backslash 1 \backslash G I N C-S C 3 \backslash F O p t \backslash R B 3 L Y P \backslash 6-31 G(d) \backslash C 7 H 8 O 1 \backslash E X I 501 \backslash 24-A p r-2005 \backslash 0 \backslash \backslash \# P$ GFIN

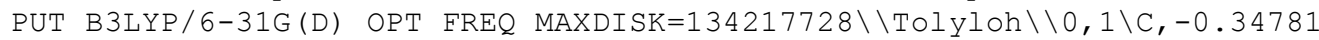
$24449,-0.0597256911,-1.0090756373 \backslash C,-0.3341822144,-0.0608917528,0.3900$ $104012 \backslash C, 0.8963018267,-0.081583047,1.0565499167 \backslash C, 2.0934733863,-0.1124$ $234726,0.3406762169 \backslash C, 2.0730432806,-0.1116750383,-1.055245909 \backslash C, 0.8491$ $682592,-0.0814396597,-1.726298899 \backslash C,-1.6239611647,-0.0868572452,1.1791$ $84047 \backslash 0,-2.6580626302,0.5434352763,0.4311279224 \backslash \mathrm{H}, 0.8252454648,-0.0741$ $772984,-2.8131495715 \backslash \mathrm{H},-1.3009722252,-0.0277457338,-1.5262136817 \backslash \mathrm{H}, 3.0$ $043036198,-0.1287358706,-1.6153610994 \backslash \mathrm{H}, 3.0410296192,-0.1268982483,0.8$ $731208978 \backslash \mathrm{H}, 0.918773396,-0.0699097629,2.1446924618 \backslash \mathrm{H},-1.8933850444,-1$. $1339764706,1.4015066487 \backslash \mathrm{H},-1.470154381,0.4144503062,2.1485745851 \backslash \mathrm{H},-3$. $4965249806,0.3670863083,0.8830055605 \backslash \backslash$ Version=Al $64 \mathrm{~T}-\mathrm{G} 03$ RevC.02 $\backslash$ State $=1$ $-\mathrm{A} \backslash \mathrm{HF}=-346.7676339 \backslash \mathrm{RMSD}=8.172 \mathrm{e}-09 \backslash \mathrm{RMSF}=2.806 \mathrm{e}-06 \backslash \mathrm{Dipole}=-0.2669156,-0$. $3323957,0.503535 \backslash P G=C 01 \quad[X(C 7 H 801)] \backslash \backslash @$

\section{$\mathrm{HO}-\mathrm{CH}\left(\mathrm{CH}_{3}\right) \mathrm{Ph}$}

$1 \backslash 1 \backslash G I N C-S C 74 \backslash F O p t \backslash R B 3 L Y P \backslash 6-31 G(d) \backslash C 8 H 1001 \backslash E X I 501 \backslash 20-A p r-2005 \backslash 0 \backslash \backslash \# P \quad G F$

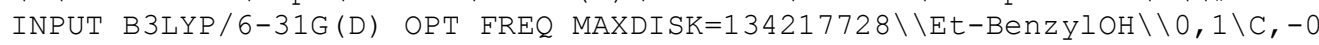
$.997424393,-0.0353659153,-0.6386261735 \backslash C,-2.1976401186,-0.0319859284,0$ $.0729190088 \backslash \mathrm{C},-2.1879154035,-0.1025045574,1.4676664167 \backslash \mathrm{C},-0.9703625415$ $,-0.1805360992,2.1464267303 \backslash \mathrm{C}, 0.228504896,-0.1887257762,1.4319403831 \backslash \mathrm{C}$ $, 0.2290432992,-0.1110386135,0.0343010002 \backslash C, 1.5389074742,-0.0822697123$, $-0.7407683037 \backslash 0,1.433288444,-0.7468430607,-2.001451827 \backslash \mathrm{C}, 1.9975105943$, $1.34307175,-1.0516791036 \backslash \mathrm{H}, 2.3138477275,-0.5697052171,-0.1254754889 \backslash \mathrm{H}$, $1.1748542166,-0.2593626995,1.9650857726 \backslash \mathrm{H},-0.9998357765,0.0120722749,-$ $1.7239153609 \backslash \mathrm{H},-0.9538642139,-0.2436233972,3.2314807932 \backslash \mathrm{H},-3.142202977$ $8,0.0248143742,-0.4622451595 \backslash \mathrm{H},-3.1229190294,-0.1016787768,2.021673741$ $3 \backslash \mathrm{H}, 2.1571750576,1.9079908618,-0.1277718733 \backslash \mathrm{H}, 1.2402885162,1.860472753$ $7,-1.6498201637 \backslash \mathrm{H}, 2.933436027,1.3181162506,-1.6193989417 \backslash \mathrm{H}, 1.089170058$ $4,-1.638222825,-1.8310784535 \backslash \backslash$ Version=Al64T-G03RevC.02 $\backslash$ State $=1-\mathrm{A} \backslash \mathrm{HF}=-3$ $86.0896528 \backslash \mathrm{RMSD}=2.806 \mathrm{e}-09 \backslash \mathrm{RMSF}=7.430 \mathrm{e}-06 \backslash \mathrm{Dipol} \mathrm{e}=-0.1521801,-0.2326452$, $0.4736137 \backslash \mathrm{PG}=\mathrm{CO} 1 \quad[\mathrm{X}(\mathrm{C} 8 \mathrm{H} 10 \mathrm{O} 1)] \backslash \backslash @$ 


\section{$\mathrm{HO}-\mathrm{C}\left(\mathrm{CH}_{3}\right)_{2} \mathrm{Ph}$}

$1 \backslash 1 \backslash G I N C-S C 2 \backslash F O p t \backslash R B 3 L Y P \backslash 6-31 G(d) \backslash C 9 H 1201 \backslash E X I 501 \backslash 19-A p r-2005 \backslash 0 \backslash \backslash \# P$ GFI

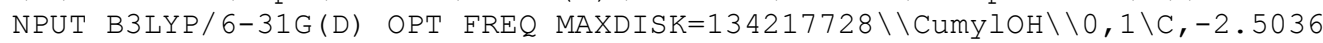
$788647,0 .,-1.4440689857 \backslash \mathrm{C},-2.4870528316,0 .,-0.0480003393 \backslash \mathrm{C},-1.27299320$ $93,0 ., 0.6376553754 \backslash \mathrm{C},-0.052922334,0 .,-0.0545128251 \backslash \mathrm{C},-0.0804037132,0 .$, $-1.4540335827 \backslash C,-1.2961772247,0 .,-2.1416288244 \backslash C, 1.2707140411,0 ., 0.713$ $1142959 \backslash \mathrm{C}, 1.3988281502,-1.2698277274,1.5754576133 \backslash \mathrm{C}, 1.3988281502,1.269$ $8277274,1.5754576133 \backslash \mathrm{H},-1.2818802773,0 ., 1.7248766849 \backslash \mathrm{H}, 0.8557550839,0$. $,-1.9990153323 \backslash \mathrm{H},-3.4200045571,0 ., 0.5099694612 \backslash \mathrm{H},-1.2959079904,0 .,-3.2$ $288416812 \backslash \mathrm{H},-3.4488457517,0 .,-1.9806594053 \backslash 0,2.3188117408,0 \ldots,-0.268206$ $7466 \backslash \mathrm{H}, 0.6028998342,1.3377132135,2.3241177985 \backslash \mathrm{H}, 0.6028998342,-1.337713$ $2135,2.3241177985 \backslash \mathrm{H}, 1.3539277532,2.1588785493,0.9394764234 \backslash \mathrm{H}, 1.3539277$ $532,-2.1588785493,0.9394764234 \backslash \mathrm{H}, 2.3585524314,1.2711090591,2.109655906$ $6 \backslash \mathrm{H}, 2.3585524314,-1.2711090591,2.1096559066 \backslash \mathrm{H}, 3.1587765443,0 ., 0.216181$ $9439 \backslash \backslash$ Version=Al64T-G03RevC.02 $\backslash$ State $=1-A^{\prime} \backslash \mathrm{HF}=-425.4036291 \backslash \mathrm{RMSD}=3.514 \mathrm{e}-$ $09 \backslash \mathrm{RMSF}=1.051 \mathrm{e}-05 \backslash \mathrm{Dipole}=0.2340133,0 ., 0.5861562 \backslash \mathrm{PG}=\mathrm{CS} \quad[\mathrm{SG}(\mathrm{C} 7 \mathrm{H} 601), \mathrm{X}(\mathrm{C} 2$ H6) $] \backslash \backslash @$

\section{$\mathrm{MeOCH}_{2} \mathrm{CN}$}

$1 \backslash 1 \backslash G I N C-A C 18 \backslash F O p t \backslash R B 3 L Y P \backslash 6-31 G(d) \backslash C 3 H 5 N 101 \backslash E X I 501 \backslash 03-A p r-2007 \backslash 0 \backslash \backslash \# P \quad g$ finput B3LYP/6-31G(d) INT (grid=ultrafine) opt freq=noraman maxdisk=134 $2177280 \backslash \backslash$ opt $\backslash \backslash 0,1 \backslash \mathrm{C}, 0.0227750665,0.0100608208,0.0377088304 \backslash 0,-0.020308$ $4408,0.072578907,1.4580196738 \backslash C, 1.243723926,-0.0246910002,2.0637890354$ $\backslash \mathrm{C}, 2.1075320671,1.1506572516,1.7990692399 \backslash \mathrm{N}, 2.7708190585,2.0742213047$, $1.5671479741 \backslash \mathrm{H}, 1.0738549173,-0.1004552315,3.1422743056 \backslash \mathrm{H}, 1.7861014608$, $-0.9280132069,1.7392805113 \backslash \mathrm{H},-1.0132114108,0.0552875508,-0.3033843578 \backslash$ $\mathrm{H}, 0.5820774091,0.8541806291,-0.3880597984 \backslash \mathrm{H}, 0.4777291524,-0.9314352094$ ,$-0.3059214986 \backslash \backslash$ Version=IA64L-G03RevD.01 \State $=1-A \backslash H F=-247.2604499 \backslash$ RMS $\mathrm{D}=3.849 \mathrm{e}-09 \backslash \mathrm{RMSF}=2.470 \mathrm{e}-05 \backslash \mathrm{Thermal}=0 . \backslash \mathrm{Dipol} \mathrm{e}=-0.4422824,-1.1336763,-0$. $1346796 \backslash P G=C 01 \quad[X(C 3 H 5 N 101)] \backslash \backslash @$

\section{$\mathrm{MeOCH}_{2} \mathrm{~F}$}

$1 \backslash 1 \backslash G I N C-A C 13 \backslash F O p t \backslash R B 3 L Y P \backslash 6-31 G(d) \backslash C 2 H 5 F 101 \backslash E X I 501 \backslash 01-A p r-2007 \backslash 0 \backslash \backslash \# P \quad g$ finput B3LYP/6-31G(d) INT (grid=ultrafine) opt freq=noraman maxdisk=134 $2177280 \backslash \backslash$ opt $\backslash \backslash 0,1 \backslash \mathrm{C},-0.037484284,0.0300768638,-0.0000213287 \backslash \mathrm{F},-0.10138$ $35664,0.028453397,1.3884005999 \backslash 0,1.2617463755,-0.0226421503,-0.4493645$ $025 \backslash \mathrm{H},-0.4614227742,0.9703359197,-0.3633833408 \backslash \mathrm{H},-0.6270527026,-0.8347$ $676121,-0.3435254516 \backslash \mathrm{C}, 1.9241699447,-1.2491402875,-0.1538260047 \backslash \mathrm{H}, 2.90$ $37375691,-1.1986773629,-0.6329808105 \backslash \mathrm{H}, 2.0496588294,-1.3810474735,0.92$ $64204307 \backslash \mathrm{H}, 1.3651040893,-2.1060875255,-0.5588275762 \backslash \backslash$ Version=IA64L-G03 RevD.01 \State=1-A \HF=-254.2643409 $\backslash$ RMSD $=9.497 e-09 \backslash$ RMSF $=5.249 e-05 \backslash$ Therma $l=0 . \backslash$ Dipole $=-0.0196797,-0.4670745,-0.3820616 \backslash P G=C 01$ [X(C2H5F1O1)] \\@

\section{$\mathrm{MeOCH}_{2} \mathrm{OH}$}

$1 \backslash 1 \backslash G I N C-A C 17 \backslash F O p t \backslash R B 3 L Y P \backslash 6-31 G(d) \backslash C 2 H 602 \backslash E X I 501 \backslash 01-A p r-2007 \backslash 0 \backslash \backslash \# P$ gfi nput B3LYP/6-31G(d) INT (grid=ultrafine) opt freq=noraman maxdisk=13421 $77280 \backslash \backslash \mathrm{opt} \backslash \backslash 0,1 \backslash \mathrm{C},-0.0527311473,0.0737587601,0.002163411 \backslash 0,-0.10445346$ $97,0.0906790342,1.4033305356 \backslash \mathrm{H}, 1.017445525,0.0691668626,-0.2418365465 \backslash$ $\mathrm{C},-1.4124514755,-0.1060107781,1.9241928537 \backslash \mathrm{H},-0.5335226464,-0.82621512$ $54,-0.4062726612 \backslash 0,-0.7253607721,1.1536226902,-0.6025652679 \backslash \mathrm{H},-0.28791$ $39157,1.9661746593,-0.2996257534 \backslash \mathrm{H},-1.324761469,-0.0850225149,3.013001$ $04 \backslash \mathrm{H},-2.1011062998,0.6816579541,1.5962346193 \backslash \mathrm{H},-1.8196509487,-1.081052$ $9839,1.6145858401 \backslash \backslash$ Version=IA64L-G03RevD.01 \State=1-A \HF=-230.2443495 RMSD $=3.401 e-09 \backslash \mathrm{RMSF}=3.126 \mathrm{e}-05 \backslash \mathrm{Thermal}=0 . \backslash \mathrm{Dipole}=0.0259893,0.0778451,0$. $1512819 \backslash P G=C 01 \quad[X(C 2 \mathrm{H} 602)] \backslash \backslash @$

\section{$\mathrm{CH}_{3} \mathrm{O}-\mathrm{CH}_{3}$}

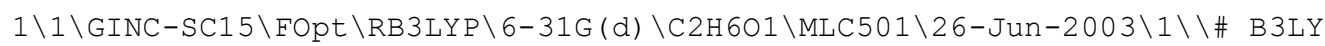


$\mathrm{P} / 6-31 \mathrm{G} * \mathrm{OPT}=(\mathrm{TIGHT}, \mathrm{MAXCYC}=100, \mathrm{Z}-\mathrm{MATRIX}) \quad \mathrm{FREQ}$ MAXDISK $=26214400 \backslash \backslash \mathrm{CH} 30 \mathrm{CH}$ $3-\mathrm{CS}-6$ dub $3 \backslash \backslash 0,1 \backslash 0 \backslash \mathrm{C}, 1, \mathrm{~B} 1 \backslash \mathrm{C}, 1, \mathrm{~B} 1,2, \mathrm{~A} 1 \backslash \mathrm{H}, 2, \mathrm{~B} 2,1, \mathrm{~A} 2,3,-180 ., 0 \backslash \mathrm{H}, 3, \mathrm{~B} 2,1, \mathrm{~A} 2$ , 2, -180. , $0 \backslash \mathrm{H}, 2, \mathrm{~B} 3,1, \mathrm{~A} 3,4, \mathrm{D} 1,0 \backslash \mathrm{H}, 3, \mathrm{~B} 3,1, \mathrm{~A} 3,5,-\mathrm{D} 1,0 \backslash \mathrm{H}, 2, \mathrm{~B} 3,1, \mathrm{~A} 3,4,-\mathrm{D} 1,0 \backslash$ $\mathrm{H}, 3, \mathrm{~B} 3,1, \mathrm{~A} 3,5, \mathrm{D} 1,0 \backslash \backslash \mathrm{B} 1=1.40986 \backslash \mathrm{B} 2=1.09336 \backslash \mathrm{B} 3=1.1029 \backslash \mathrm{A} 1=112.3117 \backslash \mathrm{A} 2=107$ $.2509 \backslash A 3=111.8183 \backslash D 1=-119.2954 \backslash \backslash$ Version=DEC-AXP-OSF/1-G03RevB.03 $\backslash$ State $=1-\mathrm{A} 1 \backslash \mathrm{HF}=-155.0250442 \backslash \mathrm{RMSD}=4.110 \mathrm{e}-09 \backslash \mathrm{RMSF}=1.445 \mathrm{e}-06 \backslash \mathrm{Dipole}=0.4163103,0$ . $0.2791602 \backslash \mathrm{PG}=\mathrm{C} 02 \mathrm{~V} \quad[\mathrm{C} 2(\mathrm{O} 1), \mathrm{SGV}(\mathrm{C} 2 \mathrm{H} 2), \mathrm{X}(\mathrm{H} 4)] \backslash \backslash \mathrm{e}$

\section{$\mathrm{CH}_{3} \mathrm{O}-\mathrm{CH}_{2} \mathrm{Ph}$}

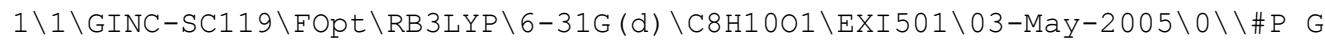

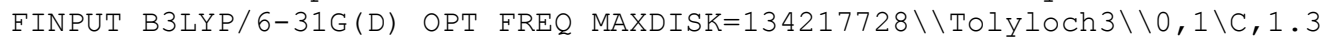
$158583439,-0.1340402532,-1.8937437 \backslash C, 0.1299949003,-0.1156037386,-1.158$ $5374539 \backslash \mathrm{C}, 0.1649938497,-0.1187688266,0.2403803394 \backslash \mathrm{C}, 1.4052245094,-0.13$ $95688131,0.8882794026 \backslash \mathrm{C}, 2.5915381292,-0.1672207157,0.1540970056 \backslash \mathrm{C}, 2.54$ $98372283,-0.1637169355,-1.2412008111 \backslash C,-1.1116519785,-0.1489004037,1.0$ $498543414 \backslash 0,-2.1570855971,0.4837652762,0.3369858485 \backslash \mathrm{C},-3.3955691296,0$. $4054734271,1.0081954803 \backslash \mathrm{H}, 1.2754616206,-0.1250962131,-2.9800724682 \backslash \mathrm{H},-$ $0.8307366389,-0.0845041422,-1.6616525979 \backslash \mathrm{H}, 3.472400983,-0.178643477,-1$ $.8155588692 \backslash \mathrm{H}, 3.5470556348,-0.1818237664,0.6720551218 \backslash \mathrm{H}, 1.4441816229,-$ $0.1308124258,1.9758934736 \backslash \mathrm{H},-1.3938528752,-1.1954794105,1.2703575388 \backslash \mathrm{H}$ $,-0.9539462173,0.3446456961,2.0245310625 \backslash \mathrm{H},-4.1345199764,0.9098368918$, $0.3802409854 \backslash \mathrm{H},-3.7103016017,-0.6394339773,1.164493853 \backslash \mathrm{H},-3.3604128914$ $, 0.9052661703,1.9898774865 \backslash \backslash$ Version=A164T-G03RevC.02 $\backslash$ State $=1-A \backslash H F=-386$ $.0780014 \backslash \mathrm{RMSD}=3.932 \mathrm{e}-09 \backslash \mathrm{RMSF}=2.111 \mathrm{e}-06 \backslash \mathrm{Dipole}=-0.202276,-0.2087115,0.4$ $033637 \backslash \mathrm{PG}=\mathrm{C} 01 \quad[\mathrm{X}(\mathrm{C} 8 \mathrm{H} 1001)] \backslash \backslash @$

\section{$\mathrm{CH}_{3} \mathrm{O}-\mathrm{CH}\left(\mathrm{CH}_{3}\right) \mathrm{Ph}$}

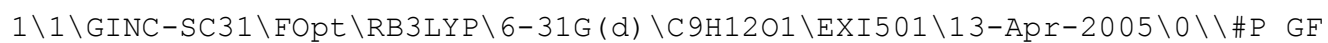
INPUT B3LYP/6-31G(D) OPT FREQ MAXDISK=134217728\\Et-BenzylOCH3 cis \\0, $1 \backslash C,-1.0156476581,1.081348342,0.1848314072 \backslash C,-2.1653107471,0.852371405$ $1,0.9472156973 \backslash C,-2.2202807931,-0.2261375255,1.8281450448 \backslash C,-1.1173645$ $595,-1.0773398784,1.9437106859 \backslash \mathrm{C}, 0.0273801216,-0.8441392719,1.18540567$ $37 \backslash C, 0.0965452492,0.2400048099,0.2961412618 \backslash \mathrm{C}, 1.391813322,0.4490313827$ $,-0.4884570778 \backslash 0,1.8407001919,-0.766348391,-1.1061933911 \backslash \mathrm{C}, 0.966591866$ $1,-1.3289664561,-2.0698593534 \backslash C, 1.3801674873,1.6208585458,-1.474453707$ $4 \backslash \mathrm{H}, 2.1881761323,0.6450455307,0.2410299334 \backslash \mathrm{H}, 0.8819999734,-1.510660049$ $5,1.2667602621 \backslash \mathrm{H},-0.9950580209,1.9264202025,-0.4969237556 \backslash \mathrm{H},-1.1504941$ $015,-1.9222375034,2.6269454292 \backslash \mathrm{H},-3.0180800809,1.5190637112,0.84722720$ $13 \backslash \mathrm{H},-3.1138349606,-0.404484619,2.4205660421 \backslash \mathrm{H}, 1.2565815369,2.57106908$ $45,-0.9439362595 \backslash \mathrm{H}, 0.5766673171,1.5436108537,-2.2141779619 \backslash \mathrm{H}, 2.3360124$ $986,1.6480221272,-2.0062629984 \backslash \mathrm{H}, 1.4855953579,-2.1956126285,-2.4882890$ $53 \backslash \mathrm{H}, 0.0168006056,-1.6570813444,-1.6261790627 \backslash \mathrm{H}, 0.7466664772,-0.634556$ $3581,-2.8932904399 \backslash \backslash$ Version=Al64T-G03RevC.02 $\backslash$ State $=1-A \backslash H F=-425.3919625$ $\backslash \mathrm{RMSD}=4.211 \mathrm{e}-09 \backslash \mathrm{RMSF}=2.018 \mathrm{e}-04 \backslash \mathrm{Dipole}=-0.388481,0.2100443,-0.1108465 \backslash \mathrm{P}$ $\mathrm{G}=\mathrm{CO} 1 \quad[\mathrm{X}(\mathrm{C} 9 \mathrm{H} 12 \mathrm{O} 1)] \backslash \backslash \mathrm{Q}$

\section{$\mathrm{CH}_{3} \mathrm{O}-\mathrm{C}\left(\mathrm{CH}_{3}\right)_{2} \mathrm{Ph}$}

$1 \backslash 1 \backslash G I N C-S C 64 \backslash F O p t \backslash R B 3 L Y P \backslash 6-31 G(d) \backslash C 10 H 1401 \backslash E X I 501 \backslash 19-A p r-2005 \backslash 0 \backslash \backslash \# P$ G

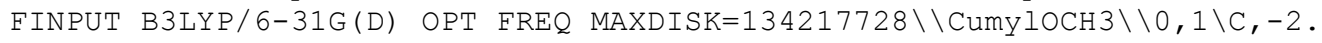
$839355779,0 .,-1.5260037515 \backslash \mathrm{C},-2.8527690729,0 .,-0.1299713064 \backslash \mathrm{C},-1.65407$ $28014,0.0 .5819408491 \backslash \mathrm{C},-0.4174018851,0 \ldots,-0.0810193781 \backslash \mathrm{C},-0.4161597631$ $, 0 \ldots,-1.4809705868 \backslash C,-1.6164103245,0 \ldots,-2.1955524901 \backslash C, 0.8910169792,0 \ldots$ $.7236269809 \backslash \mathrm{C}, 0.9705994219,-1.2712118005,1.5926337555 \backslash \mathrm{C}, 0.9705994219,1$ $.2712118005,1.5926337555 \backslash \mathrm{H},-1.689644747,0 ., 1.6685525016 \backslash \mathrm{H}, 0.5309458195$ $, 0 .,-2.0056883774 \backslash \mathrm{H},-3.7974848523,0 ., 0.4078934644 \backslash \mathrm{H},-1.5909503891,0 .,-$ $3.2825123094 \backslash \mathrm{H},-3.7723693191,0 \ldots,-2.0834564048 \backslash 0,1.9421459039,0 .,-0.257$ $2895375 \backslash \mathrm{H}, 0.1079083001,1.340518148,2.2617004602 \backslash \mathrm{H}, 0.1079083001,-1.3405$ $18148,2.2617004602 \backslash \mathrm{H}, 0.9809294247,2.1602643742,0.9541672968 \backslash \mathrm{H}, 0.980929$ $4247,-2.1602643742,0.9541672968 \backslash \mathrm{H}, 1.8706086456,1.275535106,2.217060410$ $6 \backslash \mathrm{H}, 1.8706086456,-1.275535106,2.2170604106 \backslash \mathrm{C}, 3.2806670421,0 ., 0.1938642$ $378 \backslash \mathrm{H}, 3.899866873,0 .,-0.7075599239 \backslash \mathrm{H}, 3.5316486043,0.8924372033,0.78406$ $93095 \backslash \mathrm{H}, 3.5316486043,-0.8924372033,0.7840693095 \backslash \backslash$ Version=Al64T-G03RevC $.02 \backslash$ State $=1-A^{\prime} \backslash \mathrm{HF}=-464.7065648 \backslash \mathrm{RMSD}=3.770 \mathrm{e}-09 \backslash \mathrm{RMSF}=1.179 \mathrm{e}-05 \backslash \mathrm{Dipole}=0$. $2629962,0 ., 0.4667745 \backslash P G=C S \quad[S G(C 8 H 601), X(C 2 H 8)] \backslash \backslash @$ 
TABLE S2: Geometries of the R•, $\mathrm{X}=\mathrm{CH}_{2}, \mathrm{CH} 2=\mathrm{CHPh}$ and $\mathrm{R}-\mathrm{XCH}_{2} \bullet$ species in Table 2.

NOTE: All species had zero imaginary frequencies, as determined from frequency calculations at the B3-LYP/6-31G(d) level

\section{$\mathrm{O}=\mathrm{CH}_{2}$}

$1 \backslash 1 \backslash G I N C-S C 32 \backslash F O p t \backslash R B 3 L Y P \backslash 6-31 G(d) \backslash C 1 H 201 \backslash M L C 501 \backslash 19-J u n-2003 \backslash 0 \backslash \backslash \# P$ B3L

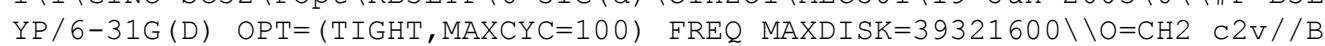
$3 \mathrm{LYP} / 6-31 \mathrm{G} * \backslash \backslash 0,1 \backslash 0,0 ., 0 \ldots,-0.6775747843 \backslash \mathrm{C}, 0 ., 0 ., 0.528906438 \backslash \mathrm{H}, 0.9377881$ $004,0.1 .1235798232 \backslash \mathrm{H},-0.9377881004,0.1 .1235798232 \backslash \backslash$ Version=DEC-AXP -0 $\mathrm{SF} / 1-\mathrm{G} 03 \mathrm{RevB} .03 \backslash \mathrm{State}=1-\mathrm{A} 1 \backslash \mathrm{HF}=-114.5004726 \backslash \mathrm{RMSD}=1.914 \mathrm{e}-09 \backslash \mathrm{RMSF}=4.379 \mathrm{e}-$ $06 \backslash \mathrm{Dipole}=0 ., 0 ., 0.8601973 \backslash \mathrm{PG}=\mathrm{C} 02 \mathrm{~V} \quad[\mathrm{C} 2(\mathrm{C} 1 \mathrm{O} 1), \mathrm{SGV}(\mathrm{H} 2)] \backslash \backslash$

\section{$\mathrm{S}=\mathrm{CH}_{2}$}

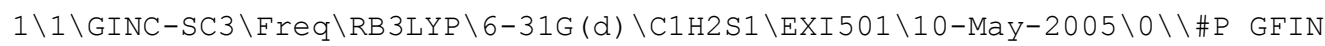
PUT B3LYP/6-31G(D) SCF=TIGHT INT (GRID=ULTRAFINE) FREQ MAXDISK=13421772 $8 \backslash \backslash \mathrm{SP} B 3 L Y P / 6-31 \mathrm{G}(\mathrm{d})$ for $\mathrm{SCH} 2 \backslash \backslash 0,1 \backslash \mathrm{S}, 0 ., 0 ., 0.5891891667 \backslash \mathrm{C}, 0 ., 0 .,-1.033$ $3508333 \backslash \mathrm{H}, 0 ., 0.925274,-1.6134608333 \backslash \mathrm{H}, 0 .,-0.925274,-1.6134608333 \backslash \backslash$ Vers ion=Al $64 \mathrm{~T}-\mathrm{G0} 3 \mathrm{RevC} .02 \backslash$ State $=1-\mathrm{A} 1 \backslash \mathrm{HF}=-437.4623164 \backslash \mathrm{RMSD}=2.345 \mathrm{e}-09 \backslash \mathrm{RMSF}=1$. $551 e-03 \backslash$ Dipole $=0 ., 0 .,-0.7485837 \backslash$ DipoleDeriv $=-0.1299865,0 ., 0 ., 0 .,-0.146$ $1157,0 ., 0 ., 0 .,-0.4176708,-0.1893008,0 ., 0 ., 0 ., 0.1767923,0 ., 0 ., 0 ., 0.5677$ $688,0.1596436,0 ., 0 ., 0 .,-0.0153383,0.070292,0 ., 0.0955525,-0.075049,0.15$ $96436,0 ., 0.0 .,-0.0153383,-0.070292,0 .,-0.0955525,-0.075049 \backslash$ Polar $=13.1$ $197517,0 ., 18.9070872,0 ., 0 ., 39.5551566 \backslash \mathrm{PG}=\mathrm{C} 02 \mathrm{~V} \quad[\mathrm{C} 2$ (C1S1), SGV (H2) ] \NImag $=0 \backslash \backslash 0.00893806,0 ., 0.02983557,0 ., 0.0 .43074475,-0.03106304,0 ., 0 ., 0.1140$ $3583,0 .,-0.05005612,0 ., 0 ., 0.57867774,0 ., 0 .,-0.38732373,0 ., 0 ., 0.6305566$ $3,0.01106249,0 ., 0 .,-0.04148639,0 ., 0 ., 0.01526355,0 ., 0.01011028,-0.00126$ $270,0 .,-0.26431081,0.11624298,0 ., 0.27129848,0 ., 0.01892332,-0.02171051$, $0 ., 0.12304154,-0.12161645,0 \ldots,-0.12847256,0.13280530,0.01106249,0 \ldots, 0 \ldots,-$ $0.04148639,0 ., 0 ., 0.01516035,0 ., 0 ., 0.01526355,0 ., 0.01011028,0.00126270$, $0 .,-0.26431081,-0.11624298,0 \ldots,-0.01709794,-0.01349229,0 ., 0.27129848,0$. $,-0.01892332,-0.02171051,0 .,-0.12304154,-0.12161645,0 ., 0.01349229,0.01$ $052166,0 ., 0.12847256,0.13280530 \backslash \backslash 0 ., 0 ., 0.00312997,0 ., 0 \ldots,-0.00428262,0$. $, 0.00016041,0.00057632,0 .,-0.00016041,0.00057632 \backslash \backslash \backslash \mathrm{e}$

\section{$\mathrm{CH}_{2}=\mathrm{CH}_{2}$}

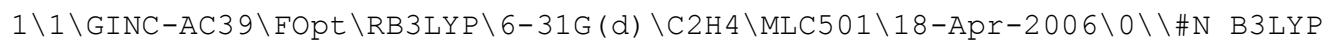

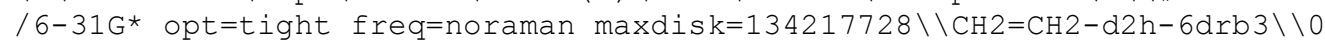
$, 1 \backslash \mathrm{C}, 0 ., 0 ., 0.0036590627 \backslash \mathrm{C}, 0 ., 0 ., 1.3346018873 \backslash \mathrm{H}, 0.9235926785,0 .,-0.5704$ $515178 \backslash \mathrm{H},-0.9235926785,0 .,-0.5704515178 \backslash \mathrm{H}, 0.9235926785,0 ., 1.9087124678$ $\backslash \mathrm{H},-0.9235926785,0.1 .9087124678 \backslash \backslash$ Version=IA64L-G03RevD.01 $\backslash$ State=1-AG $\mathrm{HF}=-78.5874583 \backslash \mathrm{RMSD}=4.475 \mathrm{e}-09 \backslash \mathrm{RMSF}=2.307 \mathrm{e}-07 \backslash \mathrm{Thermal}=0 . \backslash \mathrm{Dipole}=0 ., 0 ., 0$ $. \backslash \mathrm{PG}=\mathrm{D} 02 \mathrm{H} \quad[\mathrm{C} 2 "(\mathrm{C} 1 . \mathrm{C} 1), \mathrm{SG}(\mathrm{H} 4)] \backslash \backslash \mathrm{C}$

\section{$\mathrm{CH}_{2}=\mathrm{CHPh}$}

$1 \backslash 1 \backslash G I N C-L C 140 \backslash F O p t \backslash R B 3 L Y P \backslash 6-31 G(d) \backslash C 8 H 8 \backslash M L C 501 \backslash 09-S e p-2003 \backslash 0 \backslash \backslash \#$ B3LYP

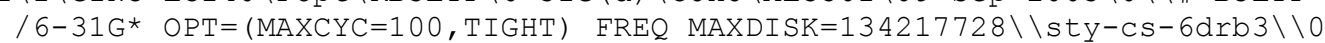
$, 1 \backslash \mathrm{H}, 0.0474426013,0 ., 4.0043693672 \backslash \mathrm{C},-0.3128916892,0 ., 2.9802150542 \backslash \mathrm{C}, 0$. $5432257252,0.1 .9507600029 \backslash \mathrm{H}, 1.6103778646,0 ., 2.1741313594 \backslash \mathrm{C}, 0.22360989$ $36,0 ., 0.5135706534 \backslash C, 1.2781058392,0 \ldots,-0.4153542904 \backslash C, 1.0335167543,0 \ldots,-$ $1.7880943466 \backslash C,-0.2778068527,0 .,-2.263496318 \backslash C,-1.3393671291,0 .,-1.353$ $1371111 \backslash \mathrm{C},-1.0928561976,0 ., 0.0163474677 \backslash \mathrm{H},-1.9311250461,0 ., 0.707157884$ $4 \backslash \mathrm{H},-2.3645874038,0 .,-1.7142937901 \backslash \mathrm{H},-0.4738733752,0 .,-3.332304318 \backslash \mathrm{H}, 1$ $.8674637892,0 .,-2.4851124697 \backslash \mathrm{H}, 2.303025796,0 .,-0.0508587581 \backslash \mathrm{H},-1.39194$ $22879,0 ., 2.8520440528 \backslash \backslash$ Version=x86-Linux-G03RevB.03\State=1-A' $\backslash H F=-309$ $.6482718 \backslash \mathrm{RMSD}=5.409 \mathrm{e}-09 \backslash \mathrm{RMSF}=3.752 \mathrm{e}-06 \backslash \mathrm{Dipole}=0.0030079,0 .,-0.0752518 \backslash$ $\mathrm{PG}=\mathrm{CS} \quad[\mathrm{SG}(\mathrm{C} 8 \mathrm{H} 8)] \backslash \backslash @$ 


\section{$\cdot \mathrm{CH}_{3}$}

$1 \backslash 1 \backslash G I N C-S C 81 \backslash F O p t \backslash U B 3 L Y P \backslash 6-31 G(d) \backslash C 1 H 3(2) \backslash E X I 501 \backslash 06-J a n-2005 \backslash 0 \backslash \backslash \# P$ GF INPUT UB3LYP/6-31G(D) SCF=TIGHT OPT=TIGHT FREQ MAXDISK=134217728 \CH3 radical optimization $\backslash \backslash 0,2 \backslash \mathrm{C}, 0 ., 0 ., 0 . \backslash \mathrm{H}, 0 .,-1.082752,0 . \backslash \mathrm{H},-0.937690738$, $0.541376,0 . \backslash \mathrm{H}, 0.937690738,0.541376,0 . \backslash \backslash$ Version=DEC-AXP-OSF/1-G0 3RevB. 0 $3 \backslash \mathrm{State}=2-\mathrm{A} 2 " \backslash \mathrm{HF}=-39.8382922 \backslash \mathrm{S} 2=0.753765 \backslash \mathrm{S} 2-1=0 . \backslash \mathrm{S} 2 \mathrm{~A}=0.750007 \backslash \mathrm{RMSD}=4.9$ $00 e-09 \backslash \mathrm{RMSF}=9.710 e-08 \backslash \mathrm{Dipole}=0 ., 0 ., 0 . \backslash \mathrm{PG}=\mathrm{D} 03 \mathrm{H} \quad[\mathrm{O}(\mathrm{C} 1), 3 \mathrm{C} 2(\mathrm{H} 1)] \backslash \backslash$

\section{$\cdot \mathrm{CH}_{2} \mathrm{CH}_{3}$}

$1 \backslash 1 \backslash G I N C-S C 116 \backslash F O p t \backslash U B 3 L Y P \backslash 6-31 G(d) \backslash C 2 H 5(2) \backslash M L C 501 \backslash 06-M a r-2003 \backslash 0 \backslash \backslash \# N \quad B$ 3LYP/6-31G(D) OPT=TIGHT FREQ MAXDISK=13107200 \\Et rad Cs $\backslash \backslash 0,2 \backslash C,-0.001$ $4599287,0.7954773663,0 . \backslash \mathrm{C}, 0.0222526364,-0.6939020591,0 . \backslash \mathrm{H},-0.994810722$ $3,-1.1263631861,0 . \backslash \mathrm{H},-0.0929966331,1.3519648812,0.9274633127 \backslash \mathrm{H},-0.0929$ $966331,1.3519648812,-0.9274633127 \backslash \mathrm{H}, 0.5280238711,-1.0935092097,0.88764$ $34386 \backslash \mathrm{H}, 0.5280238711,-1.0935092097,-0.8876434386 \backslash \backslash$ Version=DEC-AXP-OSF / 1-G98RevA.11.3 SState $=2-A^{\prime} \backslash \mathrm{HF}=-79.1578673 \backslash \mathrm{S} 2=0.753921 \backslash \mathrm{S} 2-1=0 . \backslash \mathrm{S} 2 \mathrm{~A}=0.750$ $01 \backslash \mathrm{RMSD}=8.620 \mathrm{e}-09 \backslash \mathrm{RMSF}=3.177 \mathrm{e}-06 \backslash \mathrm{Dipole}=-0.0504824,-0.0860887,0 . \backslash \mathrm{PG}=\mathrm{CS}$ $[\mathrm{SG}(\mathrm{C} 2 \mathrm{H} 1), \mathrm{X}(\mathrm{H} 4)] \backslash \backslash$

\section{$\cdot \mathrm{CH}\left(\mathrm{CH}_{3}\right)_{2}$}

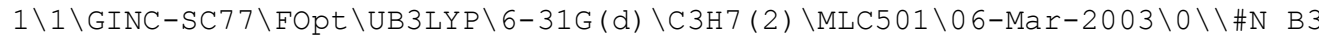

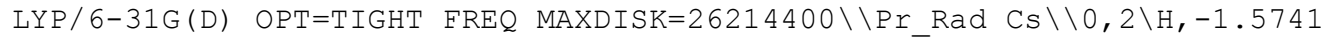
$282257,0.3384466025,0 . \backslash C,-0.4949973207,0.2014373026,0 . \backslash C, 0.1885698354$, $-0.059540887,-1.3011394423 \backslash \mathrm{C}, 0.1885698354,-0.059540887,1.3011394423 \backslash \mathrm{H}$, $-0.3378996345,0.4075090427,-2.1413392395 \backslash \mathrm{H},-0.3378996345,0.4075090427$, $2.1413392395 \backslash \mathrm{H}, 1.2217611291,0.3159557584,-1.2963791248 \backslash \mathrm{H}, 1.2217611291$, $0.3159557584,1.2963791248 \backslash \mathrm{H}, 0.256775568,-1.1397546885,-1.5289592366 \backslash \mathrm{H}$, $0.256775568,-1.1397546885,1.5289592366 \backslash \backslash$ Version=DEC-AXP-OSE / 1-G98RevA. 11.3 $\backslash$ State $=2-A^{\prime} \backslash H F=-118.4781543 \backslash \mathrm{S} 2=0.754002 \backslash \mathrm{S} 2-1=0 . \backslash \mathrm{S} 2 \mathrm{~A}=0.750011 \backslash \mathrm{RMSD}=$ $5.398 e-09 \backslash \mathrm{RMSF}=2.170 e-06 \backslash \mathrm{Dipole}=0.014982,-0.0786699,0 . \backslash \mathrm{PG}=\mathrm{CS} \quad[\mathrm{SG}(\mathrm{C} 1 \mathrm{H} 1)$ $, \mathrm{X}(\mathrm{C} 2 \mathrm{H} 6)] \backslash \backslash$

\section{$\cdot \mathrm{C}\left(\mathrm{CH}_{3}\right)_{3}$}

$1 \backslash 1 \backslash G I N C-A C 35 \backslash F O p t \backslash U B 3 L Y P \backslash 6-31 G(d) \backslash C 4 H 9(2) \backslash J O H 501 \backslash 31-J u 1-2006 \backslash 0 \backslash \backslash \#$ UB3

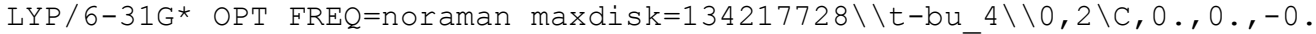
$0176048441 \backslash C, 1.4891715114,0 .,-0.176787351 \backslash C,-0.7445857557,-1.289660359$ $5,-0.176787351 \backslash \mathrm{C},-0.7445857557,1.2896603595,-0.176787351 \backslash \mathrm{H},-0.20390525$ $32,2.130303493,0.2766588458 \backslash \mathrm{H}, 1.9468495693,-0.8885646173,0.2766588458 \backslash$ $\mathrm{H},-1.7429443161,-1.2417388758,0.2766588458 \backslash \mathrm{H},-1.7429443161,1.241738875$ $8,0.2766588458 \backslash \mathrm{H}, 1.9468495693,0.8885646173,0.2766588458 \backslash \mathrm{H},-0.203905253$ $2,-2.130303493,0.2766588458 \backslash \mathrm{H},-0.8979136693,1.5552320961,-1.2404498954$ $\backslash \mathrm{H}, 1.7958273387,0 .,-1.2404498954 \backslash \mathrm{H},-0.8979136693,-1.5552320961,-1.2404$ $498954 \backslash \backslash$ Version=IA6 4L-G03RevD.01 \State $=2-\mathrm{A} 1 \backslash \mathrm{HF}=-157.7983286 \backslash \mathrm{S} 2=0.75401$ $8 \backslash \mathrm{S} 2-1=0 . \backslash \mathrm{S} 2 \mathrm{~A}=0.750012 \backslash \mathrm{RMSD}=7.741 \mathrm{e}-09 \backslash \mathrm{RMSF}=1.499 \mathrm{e}-05 \backslash$ Thermal=0. $\backslash \mathrm{Dipole}$ $=0 ., 0 .,-0.0761405 \backslash \mathrm{PG}=\mathrm{C} 03 \mathrm{~V}[\mathrm{C} 3(\mathrm{C} 1), 3 \mathrm{SGV}(\mathrm{C} 1 \mathrm{H} 1), \mathrm{X}(\mathrm{H} 6)] \backslash \backslash \mathrm{e}$

\section{$\mathrm{CH}_{3} \mathrm{OCH}_{2}{ }^{\bullet}$}

$1 \backslash 1 \backslash \mathrm{GINC}-\mathrm{SC} 32 \backslash \mathrm{FOpt} \backslash \mathrm{UB} 3 \mathrm{LYP} \backslash 6-31 \mathrm{G}(\mathrm{d}) \backslash \mathrm{C} 2 \mathrm{H} 5 \mathrm{O}$ (2) $\backslash \mathrm{MLC5} 01 \backslash 19-\mathrm{Jun}-2003 \backslash 0 \backslash \backslash \# \mathrm{P}$ B3LYP $/ 6-31 \mathrm{G}(\mathrm{D}) \quad \mathrm{OPT}=(\mathrm{TIGHT}, \mathrm{MAXCYC}=100) \quad \mathrm{FREQ}$ MAXDISK=39321600 \} \backslash \mathrm { CH } 3 - \mathrm { O } - \mathrm { CH } 2 * $\mathrm{rad} \mathrm{C} 1 / / \mathrm{B} 3 \mathrm{LYP} / 6-31 \mathrm{G}^{*} \backslash \backslash 0,2 \backslash 0,0.4671646997,-0.0176315535,-0.2913338412$ $\backslash \mathrm{C}, 0.6194032129,-0.0265127707,1.0596573999 \backslash \mathrm{H}, 1.6494088981,-0.170363840$ $7,1.3662138083 \backslash \mathrm{H},-0.1928447207,-0.4351230146,1.6615236809 \backslash \mathrm{C},-0.8752829$ $671,0.0919877531,-0.7407955798 \backslash \mathrm{H},-0.8511891749,0.035423464,-1.83104410$ $98 \backslash \mathrm{H},-1.3170075172,1.0483426656,-0.4320684734 \backslash \mathrm{H},-1.4904065574,-0.73007$ $6741,-0.3471250973 \backslash \backslash$ Version=DEC-AXP-OSF $/ 1-G 03$ RevB.03 $\backslash$ State $=2-A \backslash H F=-154$ $.3618223 \backslash \mathrm{S} 2=0.752855 \backslash \mathrm{S} 2-1=0 . \backslash \mathrm{S} 2 \mathrm{~A}=0.750006 \backslash \mathrm{RMSD}=4.394 \mathrm{e}-09 \backslash \mathrm{RMSF}=1.576 \mathrm{e}-0$ $6 \backslash \mathrm{Dipole}=-0.5149906,-0.1186528,-0.0063501 \backslash \mathrm{PG}=\mathrm{C} 01 \quad[\mathrm{X}(\mathrm{C} 2 \mathrm{H} 5 \mathrm{O} 1)] \backslash \backslash @$ 


\section{$\mathrm{CH}_{3} \mathrm{CH}_{2} \mathrm{OCH}_{2} \bullet$}

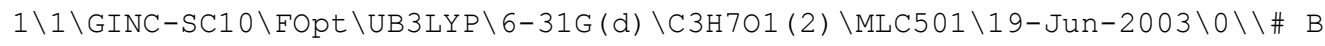
$3 \mathrm{LYP} / 6-31 \mathrm{G} * \mathrm{OPT}=(\mathrm{TIGHT}, \mathrm{MAXCYC}=100) \quad \mathrm{FREQ}$ MAXDISK=104857600 \Et-O-CH2* $r$ ad $\mathrm{C} 1 / / \mathrm{B} 3 \mathrm{LYP} / 6-31 \mathrm{G} * \backslash \backslash 0,2 \backslash 0,0.6938621591,-0.0198210446,0.2752231968 \backslash \mathrm{C}, 0$ $.9081569071,-0.0764098543,1.6161807757 \backslash \mathrm{H}, 1.9519211714,-0.2220818028,1$. $8715163223 \backslash \mathrm{H}, 0.1273699553,-0.5131527525,2.2393794915 \backslash \mathrm{C},-0.6744532668,0$ $.0903891572,-0.1202300544 \backslash \mathrm{C},-0.7378735062,0.0608558021,-1.6368977092 \backslash \mathrm{H}$ $,-1.0928004072,1.0261337093,0.2765365914 \backslash \mathrm{H},-1.2463332614,-0.7435972781$ $, 0.3154594932 \backslash \mathrm{H},-1.7768398555,0.1458954176,-1.9735439354 \backslash \mathrm{H},-0.16641452$ $56,0.8919166662,-2.0622785776 \backslash \mathrm{H},-0.3227811552,-0.8755562332,-2.0231730$ $323 \backslash \backslash$ Version $=\mathrm{DEC}-\mathrm{AXP}-\mathrm{OSF} / 1-\mathrm{G} 03 \mathrm{RevB} .03 \backslash$ State $=2-\mathrm{A} \backslash \mathrm{HF}=-193.681198 \backslash \mathrm{S} 2=0.75$ $2877 \backslash \mathrm{S} 2-1=0 . \backslash \mathrm{S} 2 \mathrm{~A}=0.750006 \backslash \mathrm{RMSD}=8.730 \mathrm{e}-09 \backslash \mathrm{RMSF}=1.246 \mathrm{e}-06 \backslash \mathrm{Dipole}=-0.4971$ $564,-0.1229149,-0.0576128 \backslash \mathrm{PG}=\mathrm{C} 01 \quad[\mathrm{X}(\mathrm{C} 3 \mathrm{H} 7 \mathrm{O} 1)] \backslash \backslash @$

\section{$\mathrm{CH}\left(\mathrm{CH}_{3}\right)_{2} \mathrm{OCH}_{2} \bullet$}

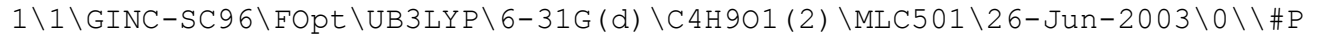
B3LYP/6-31G(D) OPT $=($ TIGHT, MAXCYC=100) FREQ MAXDISK=39321600 \\Pr-O-CH2 * $\mathrm{rad} \mathrm{C} 1 / / \mathrm{B} 3 \mathrm{LYP} / 6-31 \mathrm{G} * \backslash \backslash 0,2 \backslash 0,0.1842948252,0.9505206995,-0.2588318536 \backslash \mathrm{C}$ $, 1.4851546945,1.1943786844,-0.5712440542 \backslash \mathrm{H}, 1.6992017903,2.2512869232,-$ $0.6873986827 \backslash \mathrm{H}, 2.0012218787,0.4634874651,-1.1955686291 \backslash \mathrm{C},-0.1872377841$ $,-0.4343841196,-0.1361162223 \backslash \mathrm{H}, 0.2760667692,-0.9838811916,-0.971167151$ $7 \backslash \mathrm{C}, 0.3181304801,-1.0039393997,1.1888763296 \backslash \mathrm{C},-1.7038307556,-0.4886732$ $964,-0.2708834724 \backslash \mathrm{H},-2.056622458,-1.5236921778,-0.2082894353 \backslash \mathrm{H},-2.1773$ $864882,0.0874345532,0.5316567242 \backslash \mathrm{H},-2.0223340465,-0.068429983,-1.22987$ $30379 \backslash \mathrm{H}, 0.0635148018,-2.0664691075,1.2743343902 \backslash \mathrm{H}, 1.4051795301,-0.8996$ $764275,1.263317816 \backslash \mathrm{H},-0.1365001882,-0.4685168626,2.0298473499 \backslash \backslash$ Version $=\mathrm{DEC}-\mathrm{AXP}-\mathrm{OSF} / 1-\mathrm{G} 03 \mathrm{RevB} .03 \backslash \mathrm{State}=2-\mathrm{A} \backslash \mathrm{HF}=-232.9996354 \backslash \mathrm{S} 2=0.752971 \backslash \mathrm{S} 2-1=0$ $. \backslash \mathrm{S} 2 \mathrm{~A}=0.750006 \backslash \mathrm{RMSD}=6.169 \mathrm{e}-09 \backslash \mathrm{RMSF}=2.344 \mathrm{e}-06 \backslash \mathrm{Dipole}=-0.0595983,-0.4947$ $106,-0.1052271 \backslash \mathrm{PG}=\mathrm{C} 01 \quad[\mathrm{X}(\mathrm{C} 4 \mathrm{H} 901)] \backslash \backslash \mathrm{e}$

\section{$\mathrm{C}\left(\mathrm{CH}_{3}\right)_{3} \mathrm{OCH}_{2} \bullet$}

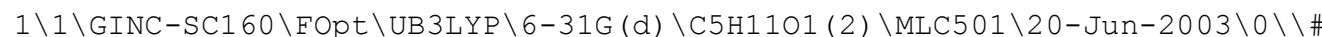
P B3LYP/6-31G(D) OPT $=(M A X C Y C=100) \quad F R E Q$ MAXDISK=134217728 GEOM=CHECK GU $\mathrm{ESS}=\mathrm{READ} \backslash \backslash \mathrm{Bu}-\mathrm{O}-\mathrm{CH} 2 * \mathrm{rad} \mathrm{C} 1 / / \mathrm{B} 3 \mathrm{LYP} / 6-31 \mathrm{G} * \backslash \backslash 0,2 \backslash 0,-0.8516671496,-0.55730$ $07725,-0.5465899956 \backslash \mathrm{C},-2.054944491,-0.1448303035,-0.0641142087 \backslash \mathrm{H},-2.88$ $65931118,-0.5976095803,-0.5932510908 \backslash \mathrm{H},-2.1534922565,0.8695579433,0.31$ $68546619 \backslash C, 0.3636100495,0.011409458,0.0134428712 \backslash C, 1.4796735242,-0.766$ $9204371,-0.6885109506 \backslash C, 0.4039873443,-0.2246359455,1.529260704 \backslash C, 0.453$ $9778468,1.5056414472,-0.3293543839 \backslash \mathrm{H}, 1.4202837181,1.9078812744,-0.0060$ $721117 \backslash \mathrm{H}, 0.3625933748,1.6534069987,-1.4104799185 \backslash \mathrm{H},-0.3286311136,2.089$ $2135398,0.1660056304 \backslash \mathrm{H}, 1.3518535288,0.1350256692,1.9449066327 \backslash \mathrm{H},-0.412$ $4630883,0.2988350871,2.0363279163 \backslash \mathrm{H}, 0.3117693379,-1.2930826772,1.74984$ $10386 \backslash \mathrm{H}, 2.4624552983,-0.4280006883,-0.3439984544 \backslash \mathrm{H}, 1.3859751266,-1.837$ $259211,-0.479349064 \backslash \mathrm{H}, 1.4217607392,-0.6235474899,-1.7724094685 \backslash \backslash$ Versio $\mathrm{n}=\mathrm{DEC}-\mathrm{AXP}-\mathrm{OSF} / 1-\mathrm{G} 03 \mathrm{RevB} .03 \backslash \mathrm{State}=2-\mathrm{A} \backslash \mathrm{HF}=-272.3135517 \backslash \mathrm{S} 2=0.753019 \backslash \mathrm{S} 2-1=$ $0 . \backslash S 2 A=0.750006 \backslash R M S D=3.984 e-09 \backslash R M S F=3.891 e-06 \backslash D i p o l e=0.3552083,0.38253$ $04,0.1719555 \backslash P G=C 01 \quad[X(C 5 H 1101)] \backslash \backslash @$

\section{$\mathrm{CH}_{3} \mathrm{SCH}_{2} \bullet$}

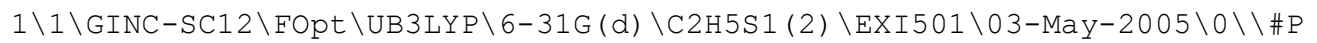
GFINPUT B3LYP/6-31G(D) OPT FREQ MAXDISK=134217728\\B3LYP 6-31G(d) for ch3sch $2 \backslash \backslash 0,2 \backslash C,-0.3612884141,-0.0209670539,-1.4326448806 \backslash S,-0.55172622$ $49,0.0044502842,0.2847229213 \backslash \mathrm{C}, 1.1886728438,0.0459538396,0.8400743505 \backslash$ $\mathrm{H},-1.2691446723,0.045470038,-2.0199140994 \backslash \mathrm{H}, 0.5408017101,-0.4234149457$ $,-1.8811213607 \backslash \mathrm{H}, 1.1805038943,0.0242433003,1.9324695189 \backslash \mathrm{H}, 1.6793381437$ $, 0.9609034076,0.4986491177 \backslash \mathrm{H}, 1.7318139442,-0.8283270624,0.469773264 \backslash \backslash \mathrm{V}$ ersion=Al64T-G03RevC.02 State $=2-A \backslash H F=-477.35164 \backslash S 2=0.754143 \backslash \mathrm{S} 2-1=0 . \backslash \mathrm{S} 2$ $\mathrm{A}=0.750013 \backslash \mathrm{RMS} \mathrm{D}=4.534 \mathrm{e}-09 \backslash \mathrm{RMSF}=5.240 \mathrm{e}-05 \backslash \mathrm{Dipole}=0.5626419,-0.1004969,0$ $.0318815 \backslash \mathrm{PG}=\mathrm{C} 01 \quad[\mathrm{X}(\mathrm{C} 2 \mathrm{H} 5 \mathrm{~S} 1)] \backslash \backslash @$

\section{$\mathrm{CH}_{3} \mathrm{CH}_{2} \mathrm{SCH}_{2} \bullet$}

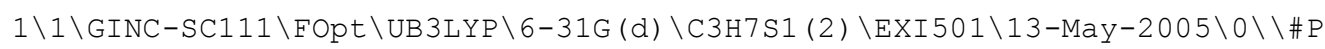


GFINPUT B3LYP/6-31G(D) OPT FREQ MAXDISK=134217728\\B3LYp opt for etsc $\mathrm{h} 2 \backslash \backslash 0,2 \backslash \mathrm{C},-0.9992978189,0.3462359524,-1.3829308084 \backslash \mathrm{S},-0.9219694013,0.2$ $137711748,0.3382562232 \backslash \mathrm{C}, 0.8947306533,0.268048519,0.630816153 \backslash \mathrm{C}, 1.6359$ $055197,-0.978803644,0.1521135953 \backslash \mathrm{H},-1.9673400068,0.1847514553,-1.84161$ $59394 \backslash \mathrm{H},-0.2330420084,0.888934269,-1.9276629294 \backslash \mathrm{H}, 1.2843168832,1.17309$ $11657,0.1514601104 \backslash \mathrm{H}, 0.9991228216,0.4003669685,1.7125298127 \backslash \mathrm{H}, 2.710847$ $1366,-0.8775057743,0.3467796644 \backslash \mathrm{H}, 1.2751108368,-1.8736468742,0.6690351$

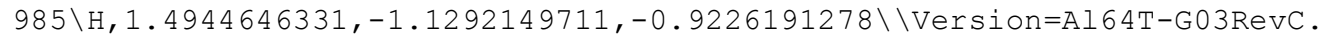
$02 \backslash \mathrm{State}=2-\mathrm{A} \backslash \mathrm{HF}=-516.6667596 \backslash \mathrm{S} 2=0.754306 \backslash \mathrm{S} 2-1=0 . \backslash \mathrm{S} 2 \mathrm{~A}=0.750014 \backslash \mathrm{RMSD}=8.3$ $51 e-09 \backslash \mathrm{RMSF}=9.684 \mathrm{e}-06 \backslash \mathrm{Dipole}=0.5820953,0.0966459,-0.056655 \backslash \mathrm{PG}=\mathrm{C} 01 \quad[\mathrm{X}(\mathrm{C}$ $3 \mathrm{H} 7 \mathrm{~S} 1)] \backslash \backslash \mathrm{e}$

\section{$\mathrm{CH}\left(\mathrm{CH}_{3}\right)_{2} \mathrm{SCH}_{2} \cdot$}

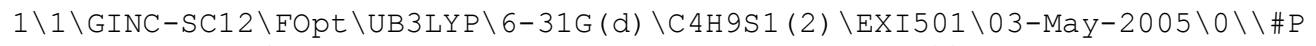
GFINPUT B3LYP/6-31G(D) OPT FREQ MAXDISK=134217728\\B3LYP 6-31G(d) for prsch $2 \backslash \backslash 0,2 \backslash C,-0.7231332155,-0.1054193024,-2.0081143585 \backslash S,-1.043580730$ $5,-0.2384972238,-0.3164504813 \backslash C, 0.6647851881,-0.1549089793,0.405706422$ $3 \backslash \mathrm{C}, 0.5734038271,-0.5678172776,1.8767129897 \backslash \mathrm{C}, 1.2854665337,1.231531750$ $5,0.2230781525 \backslash \mathrm{H},-1.5793389184,0.0314793139,-2.6577546289 \backslash \mathrm{H}, 0.20674497$ $59,-0.4801489947,-2.4245700296 \backslash \mathrm{H}, 1.2564497379,-0.8986327805,-0.1420571$ $75 \backslash \mathrm{H}, 1.5711335743,-0.5486239903,2.3303728388 \backslash \mathrm{H},-0.0633057105,0.1235377$ $959,2.4410785537 \backslash \mathrm{H}, 0.1656774204,-1.5774791035,1.9879443061 \backslash \mathrm{H}, 2.3151490$ $89,1.2353760219,0.6036129889 \backslash \mathrm{H}, 1.3072662373,1.5214433378,-0.8315830024$ $\backslash \mathrm{H}, 0.7143812828,1.988686833,0.7718646134 \backslash \backslash$ Version=Al64T-G03RevC.02\Sta $\mathrm{te}=2-\mathrm{A} \backslash \mathrm{HF}=-555.9817771 \backslash \mathrm{S} 2=0.754337 \backslash \mathrm{S} 2-1=0 . \backslash \mathrm{S} 2 \mathrm{~A}=0.750014 \backslash \mathrm{RMSD}=4.194 \mathrm{e}-09$ $\backslash \mathrm{RMSF}=3.162 \mathrm{e}-05 \backslash \mathrm{Dipole}=0.5996469,-0.0543708,0.1233131 \backslash \mathrm{PG}=\mathrm{C} 01 \quad[\mathrm{X}(\mathrm{C} 4 \mathrm{H} 9 \mathrm{~S} 1$ )$] \backslash \backslash @$

\section{$\mathrm{C}\left(\mathrm{CH}_{3}\right)_{3} \mathrm{SCH}_{2} \bullet$}

$1 \backslash 1 \backslash G I N C-S C 12 \backslash$ FOpt \UB3LYP \6-31G(d) \C5H11S1 (2) \EXI501\03-May-2005 $0 \backslash \backslash \# P$ GFINPUT B3LYP/6-31G(D) OPT FREQ MAXDISK=134217728\\B3LYP 6-31G(d) for busch $2 \backslash \backslash 0,2 \backslash C,-0.9033314404,-0.0684087223,-2.0323643839 \backslash S,-1.24181018$ $97,0.0101908533,-0.3425054785 \backslash C, 0.4488752252,0.0167641485,0.4752823518$ $\backslash \mathrm{C}, 1.1918327386,-1.2936955201,0.181193742 \backslash \mathrm{C}, 0.1474113087,0.1310826996$, $1.9775624864 \backslash \mathrm{C}, 1.2547549106,1.2294763324,-0.008878257 \backslash \mathrm{H},-1.7361220847$, $0.1326147998,-2.6960033319 \backslash \mathrm{H},-0.0390041009,-0.5980729612,-2.4175333805$ $\backslash \mathrm{H}, 2.1571417426,-1.2958366069,0.7042110854 \backslash \mathrm{H}, 0.6162778306,-2.160675716$ $2,0.5205678491 \backslash \mathrm{H}, 1.398548281,-1.4164081417,-0.8869517749 \backslash \mathrm{H}, 2.235878313$ $5,1.2443763363,0.483905703 \backslash \mathrm{H}, 1.4166177888,1.194464882,-1.0906031363 \backslash \mathrm{H}$, $0.7383710507,2.1657023472,0.2258305073 \backslash \mathrm{H}, 1.0883259814,0.1409246118,2.5$ $410651487 \backslash \mathrm{H},-0.3945650186,1.054773136,2.2070177528 \backslash \mathrm{H},-0.4497632055,-0$. $7162299682,2.3318055981 \backslash \backslash$ Version=Al64T-G03RevC.02 \State=2-A \HF=-595.29 $49393 \backslash S 2=0.754477 \backslash S 2-1=0 . \backslash S 2 A=0.750014 \backslash R M S D=6.681 e-09 \backslash R M S F=3.369 e-06 \backslash D$ ipole $=0.6039052,-0.0973427,0.1629819 \backslash \mathrm{PG}=\mathrm{C0} 1 \quad[\mathrm{X}(\mathrm{C} 5 \mathrm{H} 11 \mathrm{~S} 1)] \backslash \backslash \mathrm{Q}$

\section{$\mathrm{CH}_{3} \mathrm{CH}_{2} \mathrm{CH}_{2} \bullet$}

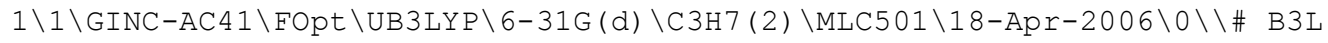
YP/6-31G* Opt=tight $F R E Q=$ noraman maxdisk=134217728 \\n-propyl-c1-6dub3 \ $\backslash 0,2 \backslash C, 0.006147324,0.0017800887,-0.0021605753 \backslash C, 1.4981256777,-0.007642$ $7603,0.0103886776 \backslash \mathrm{C},-0.5990783371,1.4131713137,0.0001401397 \backslash \mathrm{H},-1.69402$ $38239,1.3761974378,0.0219215705 \backslash \mathrm{H}, 2.0521026183,0.7807193185,0.51281503$ $86 \backslash \mathrm{H}, 2.0565476136,-0.8741667107,-0.3303118251 \backslash \mathrm{H},-0.383513452,-0.548911$ $5225,0.8755393942 \backslash \mathrm{H},-0.3621315177,-0.5600661665,-0.8728395877 \backslash \mathrm{H},-0.295$ $3523794,1.9723033422,-0.8919222665 \backslash \mathrm{H},-0.2659237234,1.9804956592,0.8776$ $994341 \backslash \backslash$ Version=IA64L-G03RevD.01 \State=2-A \HF=-118.4713715 SS =0.753881 $\backslash \mathrm{S} 2-1=0 . \backslash \mathrm{S} 2 \mathrm{~A}=0.75001 \backslash \mathrm{RMSD}=6.718 \mathrm{e}-09 \backslash \mathrm{RMSF}=1.766 \mathrm{e}-06 \backslash \mathrm{Thermal}=0 . \backslash \mathrm{Dipole}=-$ $0.0841913,-0.034525,0.0386602 \backslash P G=C 01 \quad[\mathrm{X}(\mathrm{C} 3 \mathrm{H} 7)] \backslash \backslash @$

\section{$\mathrm{CH}_{3} \mathrm{CH}_{2} \mathrm{CH}_{2} \mathrm{CH}_{2} \cdot$}

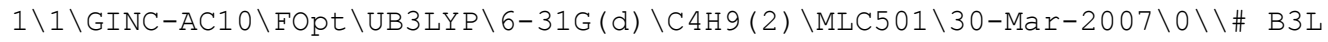
YP/6-31G* Opt=tight INT (grid=ultrafine) $F R E Q=$ noraman maxdisk $=402653184$ $\backslash \backslash \mathrm{n}$-butyl-geom $\backslash \backslash 0,2 \backslash \mathrm{C},-1.3652570459,0.4834410894,0.0221084163 \backslash \mathrm{C},-2.604$ $8935735,-0.3451075784,-0.0186807949 \backslash C,-0.0663129583,-0.3387161185,-0.0$ 
$427950878 \backslash \mathrm{C}, 1.1958216078,0.5244460693,0.0407613662 \backslash \mathrm{H},-2.6269590203,-1$. $3296241261,0.4407453653 \backslash \mathrm{H},-3.5484356286,0.0705427828,-0.3583288292 \backslash \mathrm{H},-$ $1.3430466132,1.0917885684,0.947937455 \backslash \mathrm{H},-1.3780130254,1.2179177954,-0$. $7972229602 \backslash \mathrm{H},-0.0576853916,-0.9212580585,-0.9734719387 \backslash \mathrm{H},-0.0667980791$ $,-1.0712932582,0.7766142462 \backslash \mathrm{H}, 1.226053586,1.0938818581,0.9780758232 \backslash \mathrm{H}$, $1.238894367,1.2454962252,-0.7849740991 \backslash \mathrm{H}, 2.1030117752,-0.0885852487,-0$ $.0059689622 \backslash \backslash$ Version=IA64L-G03RevD.01 \State $=2-A \backslash H F=-157.7852838 \backslash S 2=0.7$ $53882 \backslash \mathrm{S} 2-1=0 . \backslash \mathrm{S} 2 \mathrm{~A}=0.75001 \backslash \mathrm{RMSD}=6.495 \mathrm{e}-09 \backslash \mathrm{RMSF}=3.103 \mathrm{e}-07 \backslash$ Therma $I=0 . \backslash \mathrm{Dip}$ $\circ \mathrm{le}=0.0610187,0.0516022,0.0379914 \backslash \mathrm{PG}=\mathrm{C} 01 \quad[\mathrm{X}(\mathrm{C} 4 \mathrm{H} 9)] \backslash \backslash @$

\section{$\mathrm{CH}\left(\mathrm{CH}_{3}\right)_{2} \mathrm{CH}_{2} \mathrm{CH}_{2}{ }^{\bullet}$}

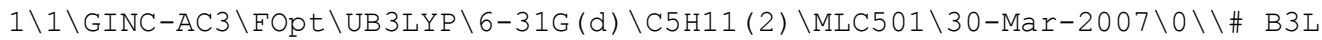
YP/6-31G* Opt=tight INT (grid=ultrafine) $F R E Q=$ noraman maxdisk=671088640

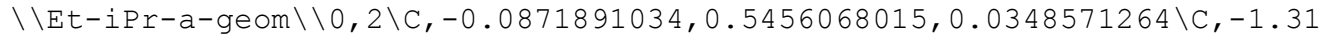
$57864908,-0.3004711853,0.0288173835 \backslash \mathrm{C}, 1.2359040372,-0.2460595507,-0.08$ $28981767 \backslash \mathrm{C}, 2.4450732145,0.6726809643,0.1383608314 \backslash \mathrm{H},-1.3375516878,-1.2$ $4163992,0.5723191059 \backslash \mathrm{H},-2.2559345746,0.0687891446,-0.3682533696 \backslash \mathrm{H},-0.0$ $457481278,1.1376256514,0.9698277825 \backslash \mathrm{H},-0.1376064449,1.2894414393,-0.77$ $44886351 \backslash \mathrm{C}, 1.3358962919,-0.976563306,-1.4291749764 \backslash \mathrm{H}, 1.2353535368,-1.0$ $039955472,0.7160126737 \backslash \mathrm{H}, 3.3853043159,0.1111515764,0.0856752665 \backslash \mathrm{H}, 2.48$ $56239797,1.4583494969,-0.6276570042 \backslash \mathrm{H}, 2.4019168977,1.1645484435,1.1176$ $499737 \backslash \mathrm{H}, 2.2512954988,-1.5773945025,-1.4877364965 \backslash \mathrm{H}, 0.4801467844,-1.64$ $25221994,-1.5855720894 \backslash \mathrm{H}, 1.3551518724,-0.2587473069,-2.2601193958 \backslash \backslash$ Ver sion=IA64L-G03RevD.01 \State $=2-A \backslash H F=-197.0994394 \backslash S 2=0.753898 \backslash S 2-1=0 . \backslash S 2$ $\mathrm{A}=0.75001 \backslash \mathrm{RMSD}=1.429 \mathrm{e}-09 \backslash \mathrm{RMSF}=3.052 \mathrm{e}-07 \backslash \mathrm{Thermal}=0 . \backslash \mathrm{Dipole}=0.0362872,0$. $0462122,0.0594878 \backslash P G=C 01 \quad[X(C 5 H 11)] \backslash \backslash @$

\section{$\mathrm{C}\left(\mathrm{CH}_{3}\right)_{3} \mathrm{CH}_{2} \mathrm{CH}_{2} \cdot$}

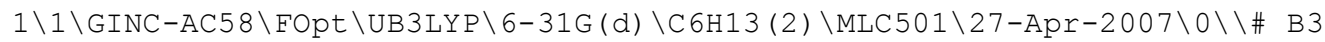
LYP/6-31G* symm=loose INT(grid=ultrafine) FREQ=noraman OPT maxdisk=402 $653184 \backslash \backslash \mathrm{Et}-\mathrm{tBu}-\mathrm{geom}-\mathrm{symm} \backslash \backslash 0,2 \backslash \mathrm{H}, 0 ., 0 ., 0 . \backslash \mathrm{C}, 0 ., 0 ., 1.097075 \backslash \mathrm{C}, 1.43734608$ $28,0 ., 1.6478708397 \backslash \mathrm{C}, 1.359368212,0 ., 3.2158810994 \backslash \mathrm{C}, 2.6614378434,0 ., 3.9$ $42480143 \backslash \mathrm{H}, 3.1754142685,0.9263687082,4.1792497129 \backslash \mathrm{H}, 3.1754142685,-0.92$ $63687082,4.1792497129 \backslash \mathrm{H}, 0.7726795652,0.8818832173,3.5124016343 \backslash \mathrm{H}, 0.772$ $6795652,-0.8818832173,3.5124016343 \backslash \mathrm{C}, 2.1740953712,1.2587645794,1.15681$ $92673 \backslash \mathrm{C}, 2.1740953712,-1.2587645794,1.1568192673 \backslash \mathrm{H}, 3.2056139872,1.28567$ $57574,1.5258160917 \backslash \mathrm{H}, 3.2056139872,-1.2856757574,1.5258160917 \backslash \mathrm{H}, 2.20886$ $83917,1.288064894,0.0607300658 \backslash \mathrm{H}, 2.2088683917,-1.288064894,0.060730065$ $8 \backslash \mathrm{H}, 1.6701280706,2.1719061396,1.498397876 \backslash \mathrm{H}, 1.6701280706,-2.1719061396$ $, 1.498397876 \backslash \mathrm{H},-0.5540789381,0.8863431131,1.4306480475 \backslash \mathrm{H},-0.5540789381$ $,-0.8863431131,1.4306480475 \backslash \backslash$ Version=IA64L-G03RevD.01 $\backslash$ State $=2-A^{\prime} \backslash H F=-2$ $36.4126912 \backslash \mathrm{S} 2=0.753984 \backslash \mathrm{S} 2-1=0 . \backslash \mathrm{S} 2 \mathrm{~A}=0.75001 \backslash \mathrm{RMSD}=3.750 \mathrm{e}-09 \backslash \mathrm{RMSF}=5.317 \mathrm{e}-$ $06 \backslash$ Thermal $=0 . \backslash$ Dipole $=0.0520039,0.0252125,0 . \backslash P G=C S \quad[S G(C 4 H 1), X(C 2 H 12)] \backslash$ $\backslash$ e

TABLE S3: Geometries of the R-CH(Ph)•, X• and R-CH(Ph)-X species in Tables 3-4.

NOTE: All species had zero imaginary frequencies, as determined from frequency calculations at the B3-LYP/6-31G(d) level

\section{$\cdot \mathrm{CH}(\mathbf{P h}) \mathrm{CH}_{3}$}

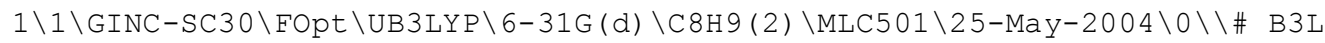

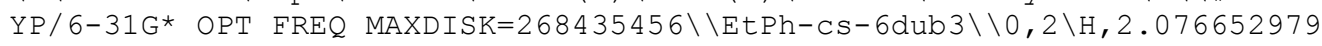
$1,-1.7071221701,0 . \backslash \mathrm{C}, 1.8330785143,-0.6472341403,0 . \backslash \mathrm{C}, 2.9648478287,0.33$ $29041964,0 . \backslash C, 0.4615044195,-0.296422334,0 . \backslash C,-2.3017509074,0.350177759$ $9,0 . \backslash C,-0.5414638247,-1.3098262805,0 . \backslash C, 0.01601966,1.0572057614,0 . \backslash C,-$ $1.3371627939,1.3659918739,0 . \backslash \mathrm{C},-1.8898621213,-0.9905058968,0 . \backslash \mathrm{H},-0.229$ $1124677,-2.3517513472,0 . \backslash \mathrm{H}, 0.7487305703,1.8588289809,0 . \backslash \mathrm{H},-1.649403422$ $1,2.4074639039,0 . \backslash \mathrm{H},-2.6317608755,-1.785156805,0 . \backslash \mathrm{H},-3.3593148857,0.59$ $85933381,0 . \backslash \mathrm{H}, 3.930913811,-0.179956881,0 . \backslash \mathrm{H}, 2.94101482,0.9926776704,0$. $8806011513 \backslash \mathrm{H}, 2.94101482,0.9926776704,-0.8806011513 \backslash \backslash$ Version=DEC-AXP-OS 
$\mathrm{F} / 1-\mathrm{G} 03 \mathrm{RevB} .03 \backslash \mathrm{State}=2-\mathrm{A} " \backslash \mathrm{HF}=-310.2333536 \backslash \mathrm{S} 2=0.779917 \backslash \mathrm{S} 2-1=0 . \backslash \mathrm{S} 2 \mathrm{~A}=0.75$ $0613 \backslash \mathrm{RMSD}=8.432 \mathrm{e}-09 \backslash \mathrm{RMSF}=3.493 \mathrm{e}-05 \backslash \mathrm{Dipole}=0.1484688,0.0260889,0 . \backslash \mathrm{PG}=\mathrm{CS}$ $[\mathrm{SG}(\mathrm{C} 8 \mathrm{H} 7), \mathrm{X}(\mathrm{H} 2)] \backslash \backslash @$

\section{$\mathrm{CH}_{3} \mathrm{CH}_{2} \mathrm{Ph}$}

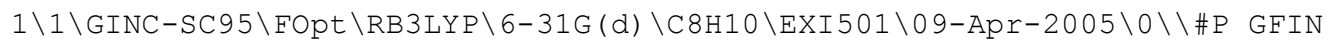

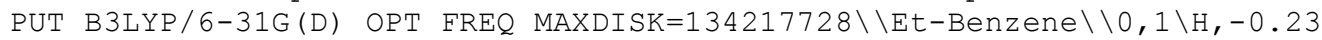
$3900392,0.7968432572,-2.9432867998 \backslash \mathrm{C},-0.2303381036,0.8033110049,-1.856$ $1810065 \backslash C,-0.2343810639,0.7989760862,0.9273260884 \backslash C,-0.234380935,-0.40$ $36009571,-1.1555963365 \backslash C,-0.2280731409,2.0153711322,-1.1635762928 \backslash C,-0$ $.2303382373,2.0091555554,0.2324009485 \backslash \mathrm{C},-0.2355670878,-0.4255633581,0$. $2456993057 \backslash \mathrm{H},-0.2412016088,-1.3440246753,-1.703293985 \backslash \mathrm{H},-0.2286339879$, $2.9565771994,-1.7069823933 \backslash \mathrm{H},-0.2339006311,2.9473834185,0.7815542238 \backslash \mathrm{C}$ $,-0.1988542231,-1.7368636812,1.0027796846 \backslash \mathrm{H},-0.2412018371,0.80308522,2$ $.0156057341 \backslash \mathrm{C}, 1.233613206,-2.224977451,1.2845928797 \backslash \mathrm{H},-0.7346565065,-2$ $.505608687,0.4315000229 \backslash \mathrm{H},-0.7346567995,-1.6264927137,1.9541719643 \backslash \mathrm{H}, 1$ $.7869924931,-2.376264702,0.3509212704 \backslash \mathrm{H}, 1.2240845842,-3.1743111998,1.8$ $326915593 \backslash \mathrm{H}, 1.7869921996,-1.4920371047,1.882446777 \backslash \backslash$ Version=Al64T-G03R evC.02 $\backslash$ State $=1-A \backslash H F=-310.8802504 \backslash R M S D=3.633 e-09 \backslash$ RMSF $=1.696 e-05 \backslash \mathrm{Dipole}=$ $0.0171231,-0.0942059,0.0543899 \backslash \mathrm{PG}=\mathrm{C} 01 \quad[\mathrm{X}(\mathrm{C} 8 \mathrm{H} 10)] \backslash \backslash \Theta$

\section{$\mathrm{CH}_{3} \mathrm{CH}_{2} \mathrm{CH}_{2} \mathrm{Ph}$}

$1 \backslash 1 \backslash G I N C-A C 25 \backslash F O p t \backslash R B 3 L Y P \backslash 6-31 G(d) \backslash C 9 H 12 \backslash E X I 501 \backslash 04-A p r-2007 \backslash 0 \backslash \backslash \# P$ gfin put B3LYP/6-31G(d) INT (grid=ultrafine) opt freq=noraman maxdisk=134217 $7280 \backslash \backslash$ opt $\backslash \backslash 0,1 \backslash \mathrm{C},-0.0789317103,0.2155105116,0.1637828005 \backslash \mathrm{C}, 0.107129391$ $3,0.5965501115,1.49980019 \backslash \mathrm{C}, 1.3425276424,0.435866064,2.1283945371 \backslash \mathrm{C}, 2$. $4192640711,-0.1108319875,1.4277636444 \backslash C, 2.2495831263,-0.493254488,0.09$ $59945711 \backslash \mathrm{C}, 1.0115874501,-0.3300353703,-0.5271784054 \backslash \mathrm{C},-1.4316769617,0$. $3519088432,-0.5019258335 \backslash \mathrm{H},-1.9597648686,1.2234322929,-0.0918501655 \backslash \mathrm{H}$, $-1.2982537432,0.5464513938,-1.5747205709 \backslash \mathrm{C},-2.320783714,-0.8963191566$, $-0.3287559238 \backslash \mathrm{H},-2.4536650865,-1.0941271298,0.7432448714 \backslash \mathrm{H},-1.79351439$ $34,-1.7694083703,-0.7357748793 \backslash C,-3.6873758051,-0.7516739134,-1.004676$ $5305 \backslash \mathrm{H}, 1.4654828178,0.7421909668,3.1642105965 \backslash \mathrm{H},-0.7256890004,1.028504$ $8102,2.0513353785 \backslash \mathrm{H}, 3.3833382113,-0.2335000788,1.9142208468 \backslash \mathrm{H}, 3.082897$ $7097,-0.9147858356,-0.4605684693 \backslash \mathrm{H}, 0.8891621163,-0.6258365556,-1.56730$ $88746 \backslash \mathrm{H},-4.2972535875,-1.6516316039,-0.866255797 \backslash \mathrm{H},-4.2469778451,0.096$ $9286784,-0.5919951987 \backslash \mathrm{H},-3.5813202934,-0.5836205392,-2.0836121027 \backslash \backslash$ Ver sion=IA 64L-G03RevD. $01 \backslash$ State $=1-A \backslash H F=-350.1942633 \backslash$ RMSD $=5.594 e-09 \backslash$ RMS $=2$. $503 e-05 \backslash$ Thermal $=0 . \backslash$ Dipole $=-0.1115861,-0.0264107,-0.0377242 \backslash P G=C 01 \quad[X(C$ $9 \mathrm{H} 12)] \backslash \backslash @$

\section{$\mathrm{CH}_{3} \mathrm{CH}_{2} \mathrm{CHFPh}$}

$1 \backslash 1 \backslash G I N C-A C 5 \backslash F O p t \backslash R B 3 L Y P \backslash 6-31 G(d) \backslash C 9 H 11 F 1 \backslash E X I 501 \backslash 05-A p r-2007 \backslash 0 \backslash \backslash \# P$ gfi nput B3LYP/6-31G(d) INT (grid=ultrafine) opt freq=noraman maxdisk=13421 $77280 \backslash \backslash \mathrm{ppt} \backslash \backslash 0,1 \backslash \mathrm{C},-0.064582806,0.1915739706,0.1340492485 \backslash \mathrm{C}, 0.078902472$ $2,0.5468633447,1.4804816901 \backslash \mathrm{C}, 1.3056389642,0.3947197978,2.1268405488 \backslash \mathrm{C}$ $, 2.4058011453,-0.1085102571,1.4293488764 \backslash C, 2.2702474674,-0.4563540596$, $0.0843711222 \backslash C, 1.0415499787,-0.3086875788,-0.5606946711 \backslash \mathrm{C},-1.412815107$ $2,0.3110782664,-0.543233974 \backslash \mathrm{H},-1.9080787308,1.2412477508,-0.2301718466$ $\backslash \mathrm{F},-1.231372593,0.4037262604,-1.9318046289 \backslash \mathrm{C},-2.3352818754,-0.87912464$ $17,-0.2691187433 \backslash \mathrm{H},-2.4670742803,-0.9652309039,0.8170643325 \backslash \mathrm{H},-1.81739$ $55072,-1.7904139318,-0.5944950248 \backslash C,-3.6918614724,-0.7558157095,-0.968$ $1355385 \backslash \mathrm{H},-4.2376730721,0.1313483159,-0.6235895285 \backslash \mathrm{H},-3.560928844,-0.6$ $697772598,-2.0508029573 \backslash \mathrm{H},-4.317079789,-1.6322102857,-0.7668972356 \backslash \mathrm{H}, 1$ $.4041122609,0.6764810689,3.1719186295 \backslash \mathrm{H},-0.7719577941,0.9498605795,2.0$ $266557142 \backslash \mathrm{H}, 3.3635844362,-0.2226753454,1.9299241856 \backslash \mathrm{H}, 3.1242838901,-0$. $8413680937,-0.4668735884 \backslash \mathrm{H}, 0.9357402917,-0.5662403724,-1.6092224643 \backslash \backslash \mathrm{V}$ ersion $=I A 64 \mathrm{~L}-\mathrm{G} 03$ RevD. $01 \backslash$ State $=1-\mathrm{A} \backslash \mathrm{HF}=-449.4269961 \backslash \mathrm{RMSD}=8.827 \mathrm{e}-09 \backslash \mathrm{RMSF}=$ $6.586 e-06 \backslash$ Thermal $=0 . \backslash$ Dipole $=-0.0902804,-0.1327448,0.5640979 \backslash P G=C 01 \quad[X($ $\mathrm{C} 9 \mathrm{H} 11 \mathrm{~F} 1)] \backslash \backslash \mathrm{Q}$ 


\section{$\cdot \mathbf{C H}(\mathbf{P h}) \mathbf{E t}$}

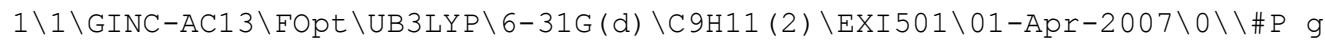
finput B3LYP/6-31G(d) INT(grid=ultrafine) opt freq=noraman maxdisk=268 $4354560 \backslash \backslash$ opt $\backslash \backslash 0,2 \backslash \mathrm{C},-0.0196841855,0 ., 0.0114958274 \backslash \mathrm{C},-0.019546042,0 ., 1$. $4138404349 \backslash \mathrm{C}, 1.2011087874,0 ., 2.1013076895 \backslash \mathrm{C}, 2.4039642682,0 ., 1.40882606$ $48 \backslash \mathrm{C}, 2.4321166048,0 .,-0.0163411789 \backslash \mathrm{C}, 1.1754873368,0 .,-0.6899266261 \backslash \mathrm{C}, 3$ $.6392621178,0 .,-0.7560299354 \backslash \mathrm{C}, 5.0174768452,0 .,-0.1628142295 \backslash \mathrm{H}, 3.56056$ $83566,0 .,-1.8421393168 \backslash \mathrm{H}, 1.1676675421,0 .,-1.7775975729 \backslash \mathrm{H}, 3.3395235267$, $0 ., 1.9602385825 \backslash \mathrm{H}, 1.2086179588,0 ., 3.1885546546 \backslash \mathrm{H},-0.9622540653,0 .,-0.5$ $302483882 \backslash \mathrm{H},-0.9573958932,0 ., 1.9620759333 \backslash \mathrm{C}, 6.1276516502,0 \ldots,-1.2190413$ $154 \backslash \mathrm{H}, 5.1437844412,-0.8739583989,0.4974371889 \backslash \mathrm{H}, 5.1437844412,0.8739583$ $989,0.4974371889 \backslash \mathrm{H}, 7.1187434103,0 .,-0.752738303 \backslash \mathrm{H}, 6.0595944973,-0.8852$ $267166,-1.862329197 \backslash \mathrm{H}, 6.0595944973,0.8852267166,-1.862329197 \backslash \backslash$ Version $=$ IA64L-G03RevD.01 \State $=2-\mathrm{A} " \backslash \mathrm{HF}=-349.5466268 \backslash \mathrm{S} 2=0.779763 \backslash \mathrm{S} 2-1=0 . \backslash \mathrm{S} 2 \mathrm{~A}=0$. $750606 \backslash \mathrm{RMSD}=7.815 \mathrm{e}-09 \backslash \mathrm{RMSF}=8.659 \mathrm{e}-05 \backslash \mathrm{Thermal}=0 . \backslash \mathrm{Dipole}=0.1585051,0 .,-0$ $.0131415 \backslash P G=C S \quad[S G(C 9 H 7), X(H 4)] \backslash \backslash @$

\section{$\mathrm{FCH}_{2} \mathrm{CH}_{2} \mathrm{Ph}$}

$1 \backslash 1 \backslash G I N C-A C 15 \backslash F O p t \backslash R B 3 L Y P \backslash 6-31 G(d) \backslash C 8 H 9 F 1 \backslash E X I 501 \backslash 03-A p r-2007 \backslash 0 \backslash \backslash \# P$ gfi

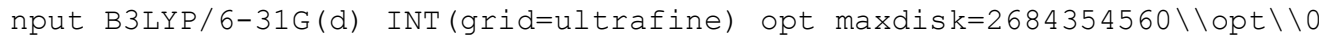
$, 1 \backslash \mathrm{C},-0.0323933893,0.1289892001,0.0924768753 \backslash \mathrm{C}, 0.0427649165,0.30447387$ $06,1.4829041754 \backslash C, 1.2654060938,0.2080977563,2.1465784583 \backslash C, 2.435724307$ $3,-0.0640550164,1.4342825713 \backslash \mathrm{C}, 2.3733169995,-0.2413068472,0.0521543329$ $\backslash \mathrm{C}, 1.1476523307,-0.1455954382,-0.6099056422 \backslash \mathrm{C},-1.3578689854,0.22885257$ $49,-0.634725468 \backslash \mathrm{H},-1.1939428884,0.1495296094,-1.717164934 \backslash \mathrm{H},-1.8206334$ $564,1.2093297258,-0.4594338601 \backslash C,-2.3774374981,-0.8365395296,-0.242391$ $1699 \backslash \mathrm{H},-1.9461085374,-1.8425745466,-0.306372161 \backslash \mathrm{F},-2.8086243603,-0.635$ $5586075,1.0696511049 \backslash \mathrm{H},-3.258946169,-0.7833844317,-0.8934279122 \backslash \mathrm{H}, 1.30$ $43010008,0.3470988047,3.2240018478 \backslash \mathrm{H},-0.8645335188,0.5054314737,2.0430$ $779972 \backslash \mathrm{H}, 3.3880579453,-0.135777685,1.9530053041 \backslash \mathrm{H}, 3.2772878647,-0.4527$ $048698,-0.5133108787 \backslash \mathrm{H}, 1.1080926695,-0.2820565307,-1.6887666897 \backslash \backslash$ Versi on $=I A 64 \mathrm{~L}-\mathrm{G} 03 \mathrm{RevD} .01 \backslash$ State $=1-\mathrm{A} \backslash \mathrm{HF}=-410.1069927 \backslash \mathrm{RMSD}=3.447 \mathrm{e}-09 \backslash \mathrm{RMSF}=5.48$ $2 \mathrm{e}-06 \backslash \mathrm{Thermal}=0 . \backslash \mathrm{Dipole}=0.1412126,-0.0361144,-0.5740307 \backslash \mathrm{PG}=\mathrm{C} 01 \quad[\mathrm{X}(\mathrm{C} 8 \mathrm{H} 9$ $\mathrm{F} 1)] \backslash \backslash \mathrm{e}$

\section{$\mathrm{FCH}_{2} \mathrm{CHFPh}$}

$1 \backslash 1 \backslash G I N C-A C 35 \backslash F O p t \backslash R B 3 L Y P \backslash 6-31 G(d) \backslash C 8 H 8 F 2 \backslash E X I 501 \backslash 09-A p r-2007 \backslash 0 \backslash \backslash \# P$ gfi

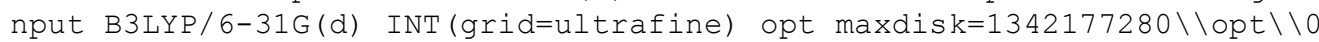
$, 1 \backslash \mathrm{C},-0.0616077085,0.2705992394,0.1211699112 \backslash \mathrm{C}, 0.1009659559,0.71161004$ $92,1.440081497 \backslash \mathrm{C}, 1.3135266301,0.5239733386,2.1031939731 \backslash \mathrm{C}, 2.3776475444$ $,-0.1017865767,1.4502135268 \backslash C, 2.2222704875,-0.5365639669,0.1330460982 \backslash$ $C, 1.0076187769,-0.3539550763,-0.5292779205 \backslash C,-1.3947157864,0.428338164$ $9,-0.5755510714 \backslash \mathrm{H},-1.8567926337,1.392398577,-0.3263523754 \backslash \mathrm{F},-1.2229787$ $159,0.3959134024,-1.9596507442 \backslash C,-2.3616178849,-0.685591509,-0.2038927$ $832 \backslash \mathrm{F},-3.6119178577,-0.425244904,-0.7432422219 \backslash \mathrm{H},-1.9930506173,-1.6392$ $693065,-0.6001154494 \backslash \mathrm{H},-2.4532807733,-0.758717124,0.8862971047 \backslash \mathrm{H}, 3.049$ $2274301,-1.0172981094,-0.3827522758 \backslash \mathrm{H}, 0.8865751594,-0.678601191,-1.557$ $3017474 \backslash \mathrm{H}, 3.3245325687,-0.2436338474,1.9640276669 \backslash \mathrm{H}, 1.4295736778,0.873$ $4131572,3.1255617366 \backslash \mathrm{H},-0.7208724245,1.2112645205,1.9493717614 \backslash \backslash$ Versio $\mathrm{n}=\mathrm{IA} 64 \mathrm{~L}-\mathrm{G} 03 \mathrm{RevD} .01 \backslash \mathrm{State}=1-\mathrm{A} \backslash \mathrm{HF}=-509.3357721 \backslash \mathrm{RMSD}=6.622 \mathrm{e}-09 \backslash \mathrm{RMSF}=5.384$ e-06\Thermal=0. \Dipole $=0.6513429,-0.1206329,0.8620365 \backslash P G=C 01 \quad[X(C 8 H 8 F 2$ ) $] \backslash \backslash 0$

\section{$\cdot \mathrm{CH}(\mathrm{Ph}) \mathrm{CH}_{2} \mathrm{~F}$}

$1 \backslash 1 \backslash G I N C-A C 20 \backslash F O p t \backslash U B 3 L Y P \backslash 6-31 G(d) \backslash C 8 H 8 F 1(2) \backslash E X I 501 \backslash 02-A p r-2007 \backslash 0 \backslash \backslash \# P$ gfinput B3LYP/6-31G(d) INT (grid=ultrafine) opt freq=noraman maxdisk=26 $84354560 \backslash \backslash$ opt $\backslash \backslash 0,2 \backslash \mathrm{C}, 0.0013908844,0.0293220083,-0.0007075677 \backslash \mathrm{C}, 0.00155$ $55775,0.0110753033,1.4009145277 \backslash C, 1.2200338047,-0.0376913846,2.0912910$ 
$612 \backslash C, 2.4242323915,-0.0645145105,1.403276237 \backslash C, 2.4506326302,-0.0300637$ $264,-0.0218328506 \backslash \mathrm{C}, 1.1978808588,0.0073643147,-0.6998954971 \backslash \mathrm{C}, 3.655401$ $6275,-0.0269655685,-0.7664936398 \backslash C, 5.0250780884,0.0483592428,-0.180722$ $9435 \backslash \mathrm{H}, 3.5829813294,-0.0120795427,-1.851260548 \backslash \mathrm{H}, 1.1927687411,0.026136$ $069,-1.7872395043 \backslash \mathrm{H}, 3.3600393273,-0.1348727772,1.9453756584 \backslash \mathrm{H}, 1.224312$ $4009,-0.0631395171,3.1779828504 \backslash \mathrm{H},-0.9399746333,0.0626475705,-0.543042$ $8326 \backslash \mathrm{H},-0.9368845829,0.0287165627,1.9479164054 \backslash \mathrm{H}, 5.7854456066,-0.09030$ $61004,-0.9572545109 \backslash \mathrm{F}, 5.237302042,-0.9324055299,0.8034245251 \backslash \mathrm{H}, 5.20598$ $25818,1.018878131,0.3061579756 \backslash \backslash$ Version=IA64L-G03RevD.01 $\backslash$ State $=2-A \backslash H F=$ $-409.457332 \backslash S 2=0.779587 \backslash S 2-1=0 . \backslash S 2 A=0.750598 \backslash R M S D=9.267 e-09 \backslash R M S F=5.601$ $e-06 \backslash$ Thermal $=0 . \backslash$ Dipole $=-0.2378297,0.4534859,-0.3816863 \backslash P G=C 01 \quad[X(C 8 H 8 F$ 1) $] \backslash \backslash @$

\section{$\mathrm{HOCH}_{2} \mathrm{CH}_{2} \mathrm{Ph}$}

$1 \backslash 1 \backslash G I N C-A C 47 \backslash F O p t \backslash R B 3 L Y P \backslash 6-31 G(d) \backslash C 8 H 1001 \backslash E X I 501 \backslash 04-A p r-2007 \backslash 0 \backslash \backslash \# P$ gf

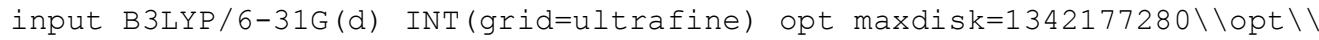
$0,1 \backslash C,-0.0081636648,-0.0447360612,-0.0181781519 \backslash C,-0.0263551177,-0.040$ $7991305,1.3856547014 \backslash \mathrm{C}, 1.1622375571,-0.0127540281,2.1188422185 \backslash \mathrm{C}, 2.391$ $8820314,0.0113687895,1.4597082221 \backslash C, 2.4247327039,0.0065872574,0.063444$ $3013 \backslash C, 1.2356735891,-0.0188473064,-0.665603641 \backslash C,-1.299233265,-0.01120$ $9026,-0.8059701595 \backslash \mathrm{H},-1.1514918574,-0.4624843883,-1.7956849479 \backslash \mathrm{H},-2.07$ $12144474,-0.5958306157,-0.291549177 \backslash C,-1.8324024833,1.4227095527,-0.99$ $78710879 \backslash \mathrm{H},-1.1070886115,2.0094113229,-1.585007329 \backslash \mathrm{H},-2.7695733545,1.3$ $930265135,-1.5631855523 \backslash 0,-2.1413833477,2.0668143322,0.2280425245 \backslash \mathrm{H},-1$ $.3138104848,2.1330481521,0.7318573333 \backslash \mathrm{H}, 1.1257068246,-0.0163071948,3.2$ $052161656 \backslash \mathrm{H},-0.9815657464,-0.0748580431,1.9045149603 \backslash \mathrm{H}, 3.3176598299,0$. $0282486927,2.0284696008 \backslash \mathrm{H}, 3.3779524933,0.0192227865,-0.4588336308 \backslash \mathrm{H}, 1$. $2706793444,-0.0249737118,-1.7531027966 \backslash \backslash$ Version=IA64L-G03RevD.01 \State $=1-\mathrm{A} \backslash \mathrm{HF}=-386.0866982 \backslash \mathrm{RMSD}=8.032 \mathrm{e}-09 \backslash \mathrm{RMSF}=7.904 \mathrm{e}-06 \backslash \mathrm{Thermal}=0 . \backslash \mathrm{Dipole}=0$ $.5888856,-0.262424,-0.1566491 \backslash \mathrm{PG}=\mathrm{C} 01 \quad[\mathrm{X}(\mathrm{C} 8 \mathrm{H} 1001)] \backslash \backslash \mathrm{Q}$

\section{$\mathrm{HOCH}_{2} \mathrm{CHFPh}$}

$1 \backslash 1 \backslash G I N C-A C 40 \backslash F O p t \backslash R B 3 L Y P \backslash 6-31 G(d) \backslash C 8 H 9 F 101 \backslash E X I 501 \backslash 06-A p r-2007 \backslash 0 \backslash \backslash \# P \quad g$ finput B3LYP/6-31G(d) INT (grid=ultrafine) opt maxdisk=1342177280 \\opt $\backslash 0,1 \backslash \mathrm{C}, 0.0957933182,0.2505441654,-0.117585078 \backslash \mathrm{C}, 0.009082042,-0.3025994$ $091,1.1655710072 \backslash C, 1.1580200078,-0.4863728909,1.9350008831 \backslash C, 2.4046574$ $013,-0.1106711949,1.4305914121 \backslash C, 2.4953670876,0.4485271527,0.154591492$ $1 \backslash C, 1.3475598894,0.6257230616,-0.618334132 \backslash C,-1.1429597746,0.382352096$ $9,-0.9661803363 \backslash \mathrm{F},-0.9805550603,1.422374801,-1.9057766839 \backslash \mathrm{H},-2.0171776$ $419,0.6439578051,-0.3578287641 \backslash C,-1.4583403189,-0.8670664449,-1.788096$ $808 \backslash \mathrm{H},-0.5717684273,-1.1211358383,-2.3916112386 \backslash \mathrm{H},-1.6638757063,-1.709$ $4751299,-1.1204323234 \backslash 0,-2.6082546411,-0.6769288508,-2.5893649773 \backslash \mathrm{H},-2$ $.4464284592,0.1340510025,-3.0987185933 \backslash \mathrm{H}, 1.0782639455,-0.9144788124,2$. $9306145485 \backslash \mathrm{H},-0.9617195004,-0.5857133573,1.5674385763 \backslash \mathrm{H}, 3.2995890249$,$0.247636559,2.0315165441 \backslash \mathrm{H}, 3.4620372365,0.7498524175,-0.2402148906 \backslash \mathrm{H}, 1$ $.4129302912,1.0714751107,-1.6055196292 \backslash \backslash$ Version=IA $64 \mathrm{~L}-\mathrm{G} 03$ RevD. $01 \backslash$ State $=1-\mathrm{A} \backslash \mathrm{HF}=-485.3190517 \backslash \mathrm{RMSD}=3.790 \mathrm{e}-09 \backslash \mathrm{RMSF}=8.232 \mathrm{e}-06 \backslash \mathrm{Thermal}=0 . \backslash \mathrm{Dipole}=0$ $.5474181,-0.057115,0.4509687 \backslash \mathrm{PG}=\mathrm{C} 01 \quad[\mathrm{X}(\mathrm{C} 8 \mathrm{H} 9 \mathrm{~F} 1 \mathrm{O} 1)] \backslash \backslash @$

\section{$\bullet \mathrm{CH}(\mathrm{Ph}) \mathrm{CH}_{2} \mathrm{OH}$}

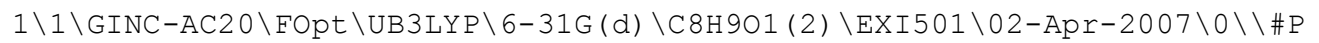
gfinput B3LYP/6-31G(d) INT(grid=ultrafine) opt freq=noraman maxdisk=26 $84354560 \backslash \backslash \mathrm{ppt} \backslash \backslash 0,2 \backslash \mathrm{C}, 0.0304376987,0.0254688839,0.0235458774 \backslash \mathrm{C}, 0.039882$ $8825,0.0394418298,1.4500213489 \backslash C, 1.2362526346,0.0010475839,2.151764144$ $\backslash C, 2.4634206574,-0.0437188034,1.4765083624 \backslash C, 2.4803377397,-0.046929862$ $2,0.0748006947 \backslash \mathrm{C}, 1.2924533788,-0.011701013,-0.638516977 \backslash \mathrm{C},-1.164524622$ $2,0.0339577004,-0.7400863628 \backslash \mathrm{C},-2.5575765301,-0.0010364322,-0.18408877$ $04 \backslash 0,-2.8304566158,1.0006837431,0.8049756655 \backslash \mathrm{H},-3.2801755001,0.0665177$ $654,-1.0114694017 \backslash \mathrm{H},-2.7531884942,-0.9514025637,0.3323497083 \backslash \mathrm{H},-2.5129$ $570146,1.8449895833,0.4462127586 \backslash \mathrm{H},-1.0643986123,0.0032746103,-1.82326$ 
$74516 \backslash \mathrm{H}, 1.3114142388,-0.0197623193,-1.7259933976 \backslash \mathrm{H},-0.904191314,0.1042$ $966459,1.9792948167 \backslash \mathrm{H}, 1.2186463486,0.0106730924,3.2387233415 \backslash \mathrm{H}, 3.42807$ $0192,-0.0794117294,-0.4565906669 \backslash \mathrm{H}, 3.3949921241,-0.07173813,2.03480481$ $35 \backslash \backslash$ Version=IA64L-G03RevD.01 \State $=2-A \backslash H F=-385.4367588 \backslash \mathrm{S} 2=0.779483 \backslash \mathrm{S} 2-$ $1=0 . \backslash \mathrm{S} 2 \mathrm{~A}=0.750597 \backslash \mathrm{RMSD}=3.729 \mathrm{e}-09 \backslash \mathrm{RMSF}=2.938 \mathrm{e}-05 \backslash \mathrm{Thermal}=0 . \backslash \mathrm{Dipole}=0.31$ $75964,0.1146841,-0.480688 \backslash \mathrm{PG}=\mathrm{CO} 1[\mathrm{X}(\mathrm{C} 8 \mathrm{H} 901)] \backslash \backslash \mathrm{e}$

\section{$\mathrm{CH}_{3} \mathrm{CHFPh}$}

$1 \backslash 1 \backslash G I N C-A C 1 \backslash F O p t \backslash R B 3 L Y P \backslash 6-31 G(d) \backslash C 8 H 9 F 1 \backslash E X I 501 \backslash 04-A p r-2007 \backslash 0 \backslash \backslash \# P$ gfin put B3LYP/6-31G(d) INT (grid=ultrafine) opt freq=noraman maxdisk=268435 $4560 \backslash \backslash \mathrm{opt} \backslash \backslash 0,1 \backslash \mathrm{C},-0.0596830412,0.1691867284,0.1284786763 \backslash \mathrm{C}, 0.052318720$ $7,0.4648948045,1.4921811989 \backslash \mathrm{C}, 1.277558584,0.3378252048,2.1465697989 \backslash \mathrm{C}$, $2.4074103745,-0.080395772,1.4403004897 \backslash C, 2.3027639402,-0.3682861934,0$. $0786142488 \backslash \mathrm{C}, 1.0756843742,-0.2453324591,-0.5749693878 \backslash \mathrm{C},-1.4051980054$, $0.2626127641,-0.5606049705 \backslash \mathrm{F},-1.215983487,0.3986237722,-1.9424254579 \backslash \mathrm{H}$ $,-1.9313828644,1.1661829425,-0.2241707784 \backslash \mathrm{C},-2.2841020132,-0.963157245$ $2,-0.3335732044 \backslash \mathrm{H}, 1.3513920183,0.5725923026,3.205205001 \backslash \mathrm{H},-0.822179366$ $1,0.8013635377,2.0461112683 \backslash \mathrm{H}, 3.3638440677,-0.1751443308,1.9474557777 \backslash$ $\mathrm{H}, 3.1796381338,-0.6868136748,-0.4791610556 \backslash \mathrm{H}, 0.9933418741,-0.456827236$ $1,-1.6357796311 \backslash \mathrm{H},-1.7725066667,-1.862550643,-0.6912853382 \backslash \mathrm{H},-3.229879$ $3798,-0.8548889382,-0.8745872133 \backslash \mathrm{H},-2.5005671626,-1.0907166304,0.73215$ $99932 \backslash \backslash$ Version=IA $64 \mathrm{~L}-\mathrm{G} 03 \mathrm{RevD} .01 \backslash$ State $=1-\mathrm{A} \backslash \mathrm{HF}=-410.1124089 \backslash \mathrm{RMSD}=4.660 \mathrm{e}-$ $09 \backslash \mathrm{RMSF}=1.086 \mathrm{e}-06 \backslash \mathrm{Thermal}=0 . \backslash \mathrm{Dipole}=-0.0807036,-0.1363995,0.5879014 \backslash \mathrm{PG}$ $=\mathrm{C} 01 \quad[\mathrm{X}(\mathrm{C} 8 \mathrm{H} 9 \mathrm{~F} 1)] \backslash \backslash \mathrm{e}$

\section{$\mathrm{NC}-\mathrm{CH}_{2} \mathrm{CH}_{2} \mathrm{Ph}$}

$1 \backslash 1 \backslash G I N C-A C 42 \backslash F O p t \backslash R B 3 L Y P \backslash 6-31 G(d) \backslash C 9 H 9 N 1 \backslash E X I 501 \backslash 03-A p r-2007 \backslash 0 \backslash \backslash \# P$ gfi

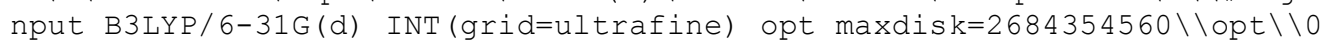
$, 1 \backslash \mathrm{C}, 0.0034951968,0.0140587532,0.000328784 \backslash \mathrm{C},-0.0021586304,0.005546154$ $, 1.4014612128 \backslash \mathrm{C}, 1.1931956717,-0.0101644417,2.1211807873 \backslash \mathrm{C}, 2.415784256$, $-0.0175838309,1.4473486302 \backslash C, 2.4347339762,-0.0070926769,0.0515128882 \backslash \mathrm{C}$ $, 1.2370128544,0.0086120641,-0.6642617952 \backslash C,-1.2943627321,-0.0078792816$ $,-0.7782520907 \backslash \mathrm{H},-1.1763326012,0.5160301106,-1.7330904144 \backslash \mathrm{H},-2.0818993$ $789,0.5138115832,-0.223496686 \backslash \mathrm{C},-1.7643623403,-1.4590936491,-1.0623271$ $553 \backslash \mathrm{H},-1.8988895787,-2.0004742493,-0.1182674852 \backslash \mathrm{H},-0.9942291539,-1.998$ $1496588,-1.6269070031 \backslash \mathrm{C},-3.0208825672,-1.5050395589,-1.8158520468 \backslash \mathrm{N},-4$ $.0165100901,-1.5171965505,-2.4128242506 \backslash \mathrm{H}, 3.3820354872,-0.0057366021,-$ $0.4811174895 \backslash \mathrm{H}, 1.2593004028,0.0245877156,-1.752100441 \backslash \mathrm{H}, 3.347546087,-0$ $.0261850216,2.0062746722 \backslash \mathrm{H}, 1.1692193791,-0.0112112314,3.2076894459 \backslash \mathrm{H},-$ $0.9514480888,0.0191183011,1.9332603808 \backslash \backslash$ Version=IA64L-G03RevD.01 \State $=1-\mathrm{A} \backslash \mathrm{HF}=-403.1184539 \backslash \mathrm{RMSD}=6.179 \mathrm{e}-09 \backslash \mathrm{RMSF}=9.174 \mathrm{e}-06 \backslash \mathrm{Thermal}=0 . \backslash \mathrm{Dipole}=1$ $.4054638,0.1415852,0.8429791 \backslash \mathrm{PG}=\mathrm{C} 01 \quad[\mathrm{X}(\mathrm{C} 9 \mathrm{H} 9 \mathrm{~N} 1)] \backslash \backslash \mathrm{Q}$

\section{$\mathrm{NC}-\mathrm{CH}_{2} \mathrm{CHFPh}$}

$1 \backslash 1 \backslash G I N C-A C 40 \backslash F O p t \backslash R B 3 L Y P \backslash 6-31 G(d) \backslash C 9 H 8 F 1 N 1 \backslash E X I 501 \backslash 12-A p r-2007 \backslash 0 \backslash \backslash \# P \quad g$ finput B3LYP/6-31G(d) INT (grid=ultrafine) opt maxdisk=1342177280 \\opt $\backslash 0,1 \backslash \mathrm{C}, 0.03035501,-0.0171295416,0.016712441 \backslash \mathrm{C}, 0.0345918068,-0.04529862$ $31,1.4160090168 \backslash C, 1.2417834704,-0.0222546238,2.1151122675 \backslash C, 2.45166996$ $87,0.0405150906,1.42181114 \backslash \mathrm{C}, 2.4500425395,0.079171437,0.0261289519 \backslash \mathrm{C}, 1$ $.2449256403,0.0486041159,-0.6754130932 \backslash C,-1.274354016,-0.0880998492,-0$ $.7425555988 \backslash \mathrm{F},-1.1299793194,0.4985649496,-2.00089295 \backslash \mathrm{H},-2.0615218685,0$ $.4600995702,-0.2116222206 \backslash C,-1.7592873924,-1.5301195116,-1.0241915168 \backslash$ $\mathrm{H},-2.6146752192,-1.4775554534,-1.7079257437 \backslash \mathrm{H},-0.9596095698,-2.0820176$ $931,-1.5311779064 \backslash \mathrm{C},-2.1585077394,-2.2482673003,0.1857481467 \backslash \mathrm{N},-2.4863$ $681499,-2.7934181568,1.1564915084 \backslash \mathrm{H}, 3.3885648381,0.1368540922,-0.51860$ $07682 \backslash \mathrm{H}, 1.2401117615,0.0929242591,-1.7596879362 \backslash \mathrm{H}, 3.3913306187,0.06437$ $71481,1.9668899287 \backslash \mathrm{H}, 1.2348190263,-0.0470837566,3.2011979652 \backslash \mathrm{H},-0.9045$ $206913,-0.0912036889,1.9614781282 \backslash \backslash$ Version=IA64L-G03RevD.01 State=1-A $\mathrm{HF}=-502.3475525 \backslash \mathrm{RMSD}=2.019 \mathrm{e}-09 \backslash \mathrm{RMSF}=8.135 \mathrm{e}-07 \backslash \mathrm{Thermal}=0 . \backslash \mathrm{Dipole}=0.5488$ 


\section{$\cdot \mathrm{CH}(\mathrm{Ph}) \mathrm{CH}_{2} \mathrm{CN}$}

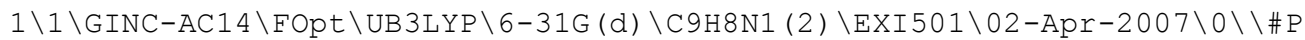
gfinput B3LYP/6-31G(d) INT(grid=ultrafine) opt freq=noraman maxdisk=26 $84354560 \backslash \backslash$ opt $\backslash \backslash 0,2 \backslash \mathrm{C},-0.0065273778,0.0006202296,0.0075605483 \backslash \mathrm{C}, 0.00329$ $13693,0.0015898418,1.409883453 \backslash C, 1.2267455264,0.0011898136,2.091803852$ $2 \backslash C, 2.4254273118,-0.0001455504,1.3924468123 \backslash C, 2.4424849405,-0.00113692$ $74,-0.0323050294 \backslash C, 1.1835015523,-0.0007190587,-0.7015158908 \backslash C, 3.638190$ $2414,-0.0025054493,-0.7863669407 \backslash C, 5.011889291,-0.003077812,-0.1646772$ $404 \backslash \mathrm{H}, 3.5754635976,-0.0033673989,-1.8696889473 \backslash \mathrm{H}, 1.1705734653,-0.00146$ $43743,-1.7886602865 \backslash \mathrm{H}, 3.3618645865,-0.0004554055,1.9430233209 \backslash \mathrm{H}, 1.2402$ $284006,0.0019244633,3.1785654587 \backslash \mathrm{H},-0.9521479053,0.000919177,-0.527972$ $0554 \backslash \mathrm{H},-0.9310894611,0.0026388234,1.9635578525 \backslash \mathrm{C}, 6.0868097687,-0.00334$ $633,-1.1621848722 \backslash \mathrm{H}, 5.1536903602,-0.8796667444,0.4873975436 \backslash \mathrm{H}, 5.154234$ $1686,0.8731728027,0.4877074546 \backslash N, 6.9321302821,-0.0041750763,-1.9570076$ $638 \backslash \backslash$ Version=IA $64 \mathrm{~L}-\mathrm{G} 03 \mathrm{RevD} .01 \backslash \mathrm{State}=2-\mathrm{A} \backslash \mathrm{HF}=-402.4684238 \backslash \mathrm{S} 2=0.780543 \backslash \mathrm{S} 2$ $-1=0 . \backslash \mathrm{S} 2 \mathrm{~A}=0.750639 \backslash \mathrm{RMSD}=9.225 \mathrm{e}-09 \backslash \mathrm{RMSF}=1.977 \mathrm{e}-05 \backslash \mathrm{Thermal}=0 . \backslash \mathrm{Dipole}=-1$. $3051862,0.0007531,1.0586634 \backslash \mathrm{PG}=\mathrm{C} 01 \quad[\mathrm{X}(\mathrm{C} 9 \mathrm{H} 8 \mathrm{~N} 1)] \backslash \backslash @$

\section{$\mathrm{CH}\left(\mathrm{CH}_{3}\right)_{2} \mathrm{CH}_{2} \mathrm{Ph}$}

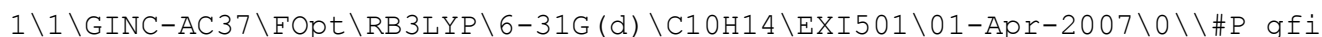
nput B3LYP/6-31G(d) INT (grid=ultrafine) opt freq=noraman maxdisk=13421 $77280 \backslash \backslash$ opt $\backslash \backslash 0,1 \backslash C,-0.0254957668,0.3076727195,0.2032067732 \backslash C, 0.32531357$ $04,0.9711612817,1.3863356533 \backslash C, 1.5573631314,0.7431080298,2.0030248125 \backslash$ $C, 2.4649155972,-0.1564683635,1.4431303287 \backslash C, 2.1312099257,-0.8234547586$ $, 0.2621045792 \backslash \mathrm{C}, 0.8995656767,-0.5908969966,-0.349339937 \backslash \mathrm{C},-1.365916073$ $6,0.5373844654,-0.4636822291 \backslash \mathrm{H},-1.8090610107,1.4703203212,-0.088739862$ $6 \backslash \mathrm{H},-1.2112786839,0.6804176886,-1.5420577039 \backslash \mathrm{C},-2.3895926966,-0.609915$ $1393,-0.2699706917 \backslash \mathrm{H},-1.9108066043,-1.5390717672,-0.6135299071 \backslash \mathrm{C},-3.63$ $23585969,-0.3677274662,-1.1382041439 \backslash \mathrm{C},-2.7763754474,-0.7949979187,1.2$ $03377268 \backslash \mathrm{H}, 1.8080131327,1.2717040475,2.9193086266 \backslash \mathrm{H},-0.3743015947,1.67$ $81276093,1.8270422684 \backslash \mathrm{H}, 3.4256194322,-0.3337324458,1.9192971489 \backslash \mathrm{H}, 2.83$ $36370419,-1.5215685457,-0.1863532601 \backslash \mathrm{H}, 0.6523618652,-1.1092642577,-1.2$ $737908876 \backslash \mathrm{H},-4.3516075048,-1.1890460151,-1.0371697251 \backslash \mathrm{H},-4.1432107126$, $0.5580766236,-0.8424858938 \backslash \mathrm{H},-3.3703561176,-0.2788472297,-2.1994625615$ $\backslash \mathrm{H},-3.4727854826,-1.6333837282,1.3227366503 \backslash \mathrm{H},-1.8999284067,-0.9923801$ $779,1.8291205593 \backslash \mathrm{H},-3.2713804129,0.1048928162,1.5927985512 \backslash \backslash$ Version=IA $64 \mathrm{~L}-\mathrm{G} 03 \mathrm{RevD} .01 \backslash \mathrm{State}=1-\mathrm{A} \backslash \mathrm{HF}=-389.5080311 \backslash \mathrm{RMSD}=6.131 \mathrm{e}-09 \backslash \mathrm{RMSF}=6.164 \mathrm{e}-06$ $\backslash$ Thermal=0. $\backslash$ Dipole $=-0.0712379,-0.0305985,-0.0488566 \backslash \mathrm{PG}=\mathrm{C} 01 \quad[\mathrm{X}(\mathrm{C} 10 \mathrm{H} 14)]$ $\backslash \backslash 0$

\section{$\mathrm{CH}\left(\mathrm{CH}_{3}\right)_{2} \mathrm{CH}(\mathrm{Ph}) \mathrm{F}$}

$1 \backslash 1 \backslash G I N C-A C 3 \backslash F O p t \backslash R B 3 L Y P \backslash 6-31 G(d) \backslash C 10 H 13 F 1 \backslash E X I 501 \backslash 04-A p r-2007 \backslash 0 \backslash \backslash \# P \quad g f$ input B3LYP/6-31G(d) INT (grid=ultrafine) opt freq=noraman maxdisk=2684 $354560 \backslash \backslash$ opt $\backslash \backslash 0,1 \backslash \mathrm{C},-0.0692730947,-0.0559817975,0.1754515748 \backslash \mathrm{C}, 0.072696$ $8056,-0.4160645958,1.519253845 \backslash C, 1.3316465171,-0.4064478746,2.12310983$ $4 \backslash C, 2.4614687619,-0.0388073303,1.3918582515 \backslash C, 2.327025604,0.3158641164$ $, 0.0475308503 \backslash C, 1.0701440832,0.3039609209,-0.5561570499 \backslash C,-1.427739541$ $2,-0.0477152585,-0.4959101518 \backslash \mathrm{H},-1.3738743339,-0.6230124287,-1.4311473$ $801 \backslash \mathrm{F},-2.3565102031,-0.712436044,0.3201929405 \backslash \mathrm{C},-1.9848191356,1.355053$ $6099,-0.8089759524 \backslash \mathrm{C},-3.2801486025,1.2422364461,-1.6262695757 \backslash \mathrm{H},-1.224$ $4101312,1.8535896278,-1.4273393495 \backslash \mathrm{C},-2.197409647,2.1834762382,0.46557$ $50866 \backslash \mathrm{H}, 3.2019510088,0.5959188695,-0.5333188498 \backslash \mathrm{H}, 0.9767951138,0.57071$ $69024,-1.6071079083 \backslash \mathrm{H}, 3.4408322696,-0.0334593515,1.8627518915 \backslash \mathrm{H}, 1.4279$ $906986,-0.6901640114,3.1679700819 \backslash \mathrm{H},-0.8050488951,-0.7115926508,2.0830$ $829803 \backslash \mathrm{H},-2.5808620682,3.1787965253,0.2156838642 \backslash \mathrm{H},-2.925380114,1.6956$ 
$736059,1.1225633662 \backslash \mathrm{H},-1.265678183,2.3095741339,1.0261498556 \backslash \mathrm{H},-3.6701$ $91577,2.2346464097,-1.8777454125 \backslash \mathrm{H},-3.1174812693,0.6968674248,-2.56405$ $54267 \backslash \mathrm{H},-4.0482967624,0.709032121,-1.0561856937 \backslash \backslash$ Version=IA64L-G03RevD $.01 \backslash$ State $=1-A \backslash H F=-488.7407058 \backslash \mathrm{RMSD}=5.165 \mathrm{e}-09 \backslash \mathrm{RMSF}=5.084 \mathrm{e}-06 \backslash$ Therma $l=0$.

$\backslash$ Dipole $=0.3525234,0.3329368,-0.2935311 \backslash \mathrm{PG}=\mathrm{C} 01 \quad[\mathrm{X}(\mathrm{C} 10 \mathrm{H} 13 \mathrm{~F} 1)] \backslash \backslash \mathrm{C}$

\section{$\cdot \mathrm{CH}(\mathrm{Ph})-\mathrm{iPr}$}

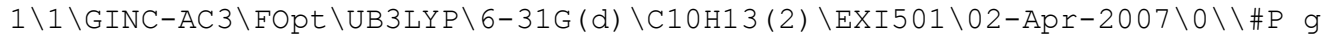
finput B3LYP/6-31G(d) INT (grid=ultrafine) opt freq=noraman maxdisk=134 $2177280 \backslash \backslash$ opt $\backslash \backslash 0,2 \backslash C, 0.023184624,0.2630135607,0.0182526247 \backslash C, 0.01685118$ $73,0.0574274774,1.3881347067 \backslash C, 1.1960541787,-0.2995420451,2.0580831542$ $\backslash \mathrm{C}, 2.3822150351,-0.4458577025,1.32679996 \backslash \mathrm{C}, 2.4000873225,-0.2435259597$, $-0.0457321065 \backslash C, 1.215587191,0.1188454204,-0.7540392033 \backslash C, 1.1753093916$, $0.3355312802,-2.1518167963 \backslash \mathrm{C}, 2.3233468326,0.2544113229,-3.1177783584 \backslash \mathrm{C}$ $, 2.9407306182,1.6516775668,-3.3611131371 \backslash C, 1.8742201016,-0.378926181,-$ $4.4476379201 \backslash \mathrm{H}, 0.2178793153,0.6425313338,-2.5739027835 \backslash \mathrm{H},-0.8959669775$ $, 0.5395078801,-0.4933146186 \backslash \mathrm{H}, 3.3337250891,-0.3601974326,-0.5872897936$ $\backslash \mathrm{H}, 3.3014663292,-0.7203500172,1.8384831636 \backslash \mathrm{H},-0.9091314069,0.174551937$ $, 1.9456300016 \backslash \mathrm{H}, 1.190273144,-0.4600726709,3.1324132787 \backslash \mathrm{H}, 3.1099571961$, $-0.3841834992,-2.6945327254 \backslash \mathrm{H}, 3.7852250144,1.5875802631,-4.0587791373 \backslash$ $\mathrm{H}, 2.1987954715,2.3352790777,-3.7915572087 \backslash \mathrm{H}, 3.3000607692,2.0932787631$, $-2.4255906336 \backslash \mathrm{H}, 2.7112042184,-0.4439561748,-5.1528174258 \backslash \mathrm{H}, 1.478216160$ $2,-1.388724539,-4.2938726157 \backslash \mathrm{H}, 1.0864845335,0.2222464535,-4.9196160192$ $\backslash \backslash$ Version=IA6 4L-G03RevD.01 \State $=2-A \backslash H F=-388.8605294 \backslash \mathrm{S} 2=0.780973 \backslash \mathrm{S} 2-1=$ $0 . \backslash \mathrm{S} 2 \mathrm{~A}=0.750657 \backslash \mathrm{RMSD}=9.868 \mathrm{e}-09 \backslash \mathrm{RMSF}=1.237 \mathrm{e}-06 \backslash \mathrm{Thermal}=0 . \backslash \mathrm{Dipole}=0.0639$ $813,0.0222005,-0.1266125 \backslash \mathrm{PG}=\mathrm{C} 01 \quad[\mathrm{X}(\mathrm{C} 10 \mathrm{H} 13)] \backslash \backslash @$

\section{$\mathrm{C}\left(\mathrm{CH}_{3}\right) \mathrm{CH}_{2} \mathrm{Ph}$}

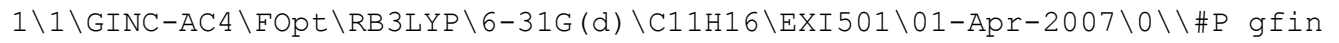
put B3LYP/6-31G(d) INT (grid=ultrafine) opt freq=noraman maxdisk=134217 $7280 \backslash \backslash$ opt $\backslash \backslash 0,1 \backslash C,-0.0150766901,-0.1813479964,0.197603837 \backslash \mathrm{C}, 0.250043602$ $8,-0.8913440776,1.3781460983 \backslash \mathrm{C}, 1.4825921975,-0.785561271,2.0241428567 \backslash$ $C, 2.4828321333,0.0324961778,1.4963924463 \backslash C, 2.2402216151,0.7373362961,0$ $.3162572046 \backslash \mathrm{C}, 1.0055402048,0.6271901457,-0.3247835436 \backslash \mathrm{C},-1.3548508003$, $-0.2980049015,-0.5009157888 \backslash C,-2.4634618223,0.7220896951,-0.0864240243$ $\backslash \mathrm{H}, 3.0151879336,1.3688203247,-0.1109217506 \backslash \mathrm{H}, 0.8319799211,1.1707041487$ $,-1.2507225072 \backslash \mathrm{H}, 3.4449808571,0.1132544811,1.9952387073 \backslash \mathrm{H}, 1.6633108863$ $,-1.3484636662,2.9364515267 \backslash \mathrm{H},-0.5173156267,-1.5421279696,1.7911398331$ $\backslash \mathrm{H},-1.2007578596,-0.199831335,-1.5840067845 \backslash \mathrm{H},-1.7541597636,-1.3080756$ $939,-0.3373131538 \backslash C,-2.8070275801,0.5819367417,1.4077715221 \backslash C,-3.71863$ $41406,0.4070816016,-0.9235817593 \backslash C,-2.0155511028,2.1664647529,-0.37529$ $7696 \backslash \mathrm{H},-4.5371738396,1.0935689865,-0.6750868765 \backslash \mathrm{H},-3.5158690067,0.5043$ $104105,-1.9974598431 \backslash \mathrm{H},-4.0730944465,-0.6150273551,-0.7407735095 \backslash \mathrm{H},-2$. $8140767872,2.8745557056,-0.1220118245 \backslash \mathrm{H},-1.1296414606,2.4384486793,0.2$ $080851385 \backslash \mathrm{H},-1.775464691,2.3038804858,-1.4374357436 \backslash \mathrm{H},-3.6136904358,1$. $2722805658,1.683253901 \backslash \mathrm{H},-3.1456894126,-0.4348488966,1.6446828879 \backslash \mathrm{H},-1$ $.9434519074,0.8057309237,2.0428112305 \backslash \backslash$ Version=IA64L-G03RevD.01 $\backslash$ State $=$ $1-\mathrm{A} \backslash \mathrm{HF}=-428.8204533 \backslash \mathrm{RMSD}=7.745 \mathrm{e}-09 \backslash \mathrm{RMSF}=1.020 \mathrm{e}-05 \backslash$ Therma $l=0 . \backslash \mathrm{Dipole}=-0$ $.0662524,0.0108749,-0.0198596 \backslash \mathrm{PG}=\mathrm{C} 01 \quad[\mathrm{X}(\mathrm{C} 11 \mathrm{H} 16)] \backslash \backslash \mathrm{Q}$

\section{$\mathrm{C}\left(\mathrm{CH}_{3}\right) \mathbf{C H}(\mathbf{P h}) \mathbf{F}$}

$1 \backslash 1 \backslash G I N C-A C 5 \backslash F O p t \backslash R B 3 L Y P \backslash 6-31 G(d) \backslash C 11 H 15 F 1 \backslash E X I 501 \backslash 05-A p r-2007 \backslash 0 \backslash \backslash \# P$ gf input B $3 L Y P / 6-31 \mathrm{G}(\mathrm{d})$ INT (grid=ultrafine) opt freq=noraman maxdisk=1342 $177280 \backslash \backslash$ opt $\backslash \backslash 0,1 \backslash \mathrm{C}, 0.1251725893,0.1571564623,-0.1351833282 \backslash \mathrm{C}, 0.1646259$ $894,-0.6874154562,0.9815458261 \backslash C, 1.3326904658,-0.8223185981,1.73295237$ $81 \backslash \mathrm{C}, 2.4754913957,-0.1005560288,1.3847530852 \backslash \mathrm{C}, 2.4387345235,0.75936810$ $38,0.2856126988 \backslash C, 1.2718677514,0.8893823018,-0.4679598414 \backslash C,-1.1430897$ $766,0.2888631824,-0.954038697 \backslash \mathrm{H},-2.0118438857,0.2034666463,-0.28632373$ $05 \backslash F,-1.1844967294,1.5852197801,-1.4977248321 \backslash C,-1.3382560313,-0.71682$ $85697,-2.1246164151 \backslash C,-2.6850417213,-0.3893404853,-2.8019837896 \backslash C,-1.3$ 
$843540236,-2.1551586049,-1.578313041 \backslash \mathrm{C},-0.2015186217,-0.5835185658,-3$. $1537334994 \backslash \mathrm{H}, 3.3195104259,1.3365343495,0.0162880571 \backslash \mathrm{H}, 1.2382342153,1.5$ $750063467,-1.3077361503 \backslash \mathrm{H}, 3.3843770083,-0.1992788219,1.9722438855 \backslash \mathrm{H}, 1$. $3451552422,-1.4820697037,2.5965481207 \backslash \mathrm{H},-0.7268636807,-1.2384066057,1$. $2719458721 \backslash \mathrm{H},-2.868642395,-1.0746469207,-3.6374964446 \backslash \mathrm{H},-3.5193771928$, $-0.4921347116,-2.0964100691 \backslash \mathrm{H},-2.6933000285,0.6336159629,-3.1881762464$ $\backslash \mathrm{H},-1.5954502712,-2.855334147,-2.3948189279 \backslash \mathrm{H},-0.4345021921,-2.4500370$ $742,-1.1212851813 \backslash \mathrm{H},-2.1763358799,-2.2776958674,-0.8281195438 \backslash \mathrm{H},-0.378$ $8076727,-1.2571322354,-4.0001828447 \backslash \mathrm{H},-0.1440224292,0.4383702239,-3.54$ $21897635 \backslash \mathrm{H}, 0.7699685343,-0.8400877826,-2.7183606816 \backslash \backslash$ Version=IA64L-G03

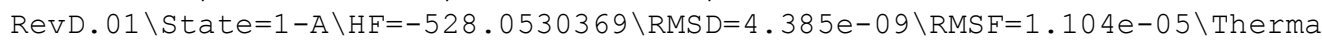
$I=0 . \backslash \mathrm{Dipole}=0.0951659,-0.5429,0.1602876 \backslash \mathrm{PG}=\mathrm{C} 01 \quad[\mathrm{X}(\mathrm{C} 11 \mathrm{H} 15 \mathrm{~F} 1)] \backslash \backslash \mathrm{e}$

\section{$\cdot \mathrm{CH}(\mathrm{Ph})-\mathrm{tBu}$}

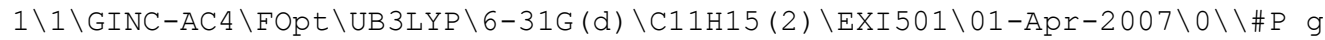
finput B3LYP/6-31G(d) INT (grid=ultrafine) opt freq=noraman maxdisk=134 $2177280 \backslash \backslash$ opt $\backslash \backslash 0,2 \backslash C,-0.0347321568,0 .,-0.0545388334 \backslash C,-0.1322719837,0$, $1.3439735133 \backslash C, 1.0402556283,0 ., 2.1091444691 \backslash C, 2.2887774789,0 ., 1.502104$ $1786 \backslash \mathrm{C}, 2.422291886,0 ., 0.0809597456 \backslash \mathrm{C}, 1.2061304598,0 \ldots,-0.6698412209 \backslash \mathrm{C}, 3$ $.6503015575,0 .,-0.6304589199 \backslash \mathrm{C}, 5.0792508675,0 .,-0.116257761 \backslash \mathrm{H}, 3.547852$ $8595,0 .,-1.7150404635 \backslash \mathrm{H}, 1.2694992519,0 .,-1.7556065998 \backslash \mathrm{H}, 3.1731326637,0$ $., 2.1276638123 \backslash \mathrm{H}, 0.9767542402,0 ., 3.1945561078 \backslash \mathrm{H},-0.9363429613,0 .,-0.66$ $20008907 \backslash \mathrm{H},-1.1047354863,0 ., 1.8279861332 \backslash \mathrm{C}, 6.0240130969,0 .,-1.33805548$ $1 \backslash \mathrm{C}, 5.38062757,-1.2693027121,0.7207238125 \backslash \mathrm{C}, 5.38062757,1.2693027121,0$. $7207238125 \backslash \mathrm{H}, 6.4284883238,-1.2674723351,1.0465478157 \backslash \mathrm{H}, 4.7525440721,-1$ $.3393165717,1.6131404274 \backslash \mathrm{H}, 5.2127019736,-2.1742693706,0.1260613195 \backslash \mathrm{H}, 7$ $.0728919826,0 .,-1.0195476331 \backslash \mathrm{H}, 5.8613660789,-0.8868279855,-1.962337426$ $9 \backslash \mathrm{H}, 5.8613660789,0.8868279855,-1.9623374269 \backslash \mathrm{H}, 6.4284883238,1.267472335$ $1,1.0465478157 \backslash \mathrm{H}, 5.2127019736,2.1742693706,0.1260613195 \backslash \mathrm{H}, 4.7525440721$ $, 1.3393165717,1.6131404274 \backslash \backslash$ Version=IA64L-G03RevD.01 $\backslash$ State $=2-A " \backslash H F=-42$ $8.1688179 \backslash \mathrm{S} 2=0.780456 \backslash \mathrm{S} 2-1=0 . \backslash \mathrm{S} 2 \mathrm{~A}=0.750633 \backslash \mathrm{RMSD}=8.883 \mathrm{e}-09 \backslash \mathrm{RMSF}=1.947 \mathrm{e}-$ $05 \backslash$ Thermal $=0 . \backslash$ Dipole $=0.1063929,0 .,-0.0152248 \backslash P G=C S \quad[S G(C 9 H 7), X(C 2 H 8)] \backslash$ $\backslash$ Q

\section{TABLE S4: Geometries of the radicals and alkoxyamines in Table 5}

NOTE: All species had zero imaginary frequencies, as determined from frequency calculations at the B3-LYP/6-31G(d) level

\section{TEMPO•}

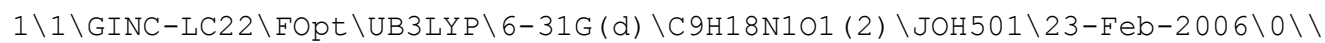
\# UB3LYP/6-31G* OPT FREQ=NORAMAN MAXDISK=78643200\\tempo $1 \mathrm{~s} \backslash \backslash 0,2 \backslash \mathrm{N}, 0$., $-0.7434209403,-0.2055568746 \backslash 0,0 .,-2.0200229887,-0.0536930208 \backslash C, 0 ., 2.12$ $91122715,0.0202793419 \backslash \mathrm{H}, 0 ., 3.1640205144,-0.3430045373 \backslash \mathrm{H}, 0 ., 2.188129084$ $3,1.1160257969 \backslash C, 1.2471385297,1.4014636869,-0.4850966271 \backslash C,-1.24713852$ $97,1.4014636869,-0.4850966271 \backslash C, 1.3316319116,-0.0694129504,-0.02866995$ $47 \backslash \mathrm{C},-1.3316319116,-0.0694129504,-0.0286699547 \backslash \mathrm{C}, 2.3409037477,-0.82546$ $55447,-0.9084597306 \backslash \mathrm{C},-2.3409037477,-0.8254655447,-0.9084597306 \backslash \mathrm{C}, 1.76$ $16105348,-0.1821678854,1.4497256469 \backslash \mathrm{C},-1.7616105348,-0.1821678854,1.44$ $97256469 \backslash \mathrm{H}, 2.1613559084,1.9128572411,-0.159270535 \backslash \mathrm{H},-2.1613559084,1.91$ $28572411,-0.159270535 \backslash \mathrm{H}, 1.2461755503,1.4303666274,-1.5834226174 \backslash \mathrm{H},-1.2$ $461755503,1.4303666274,-1.5834226174 \backslash \mathrm{H}, 2.0267037652,-0.8094181301,-1.9$ $575486217 \backslash \mathrm{H},-2.0267037652,-0.8094181301,-1.9575486217 \backslash \mathrm{H}, 3.3238440946,-$ $0.3468698717,-0.8343424769 \backslash \mathrm{H},-3.3238440946,-0.3468698717,-0.8343424769$ $\backslash \mathrm{H}, 2.4264203456,-1.8666991071,-0.5927537894 \backslash \mathrm{H},-2.4264203456,-1.8666991$ $071,-0.5927537894 \backslash \mathrm{H}, 2.801008229,0.1459162404,1.5646144668 \backslash \mathrm{H},-2.8010082$ $29,0.1459162404,1.5646144668 \backslash \mathrm{H}, 1.1414032892,0.4302012714,2.1114446532 \backslash$ $\mathrm{H},-1.1414032892,0.4302012714,2.1114446532 \backslash \mathrm{H}, 1.6868923818,-1.2242044777$ $, 1.7731554021 \backslash \mathrm{H},-1.6868923818,-1.2242044777,1.7731554021 \backslash \backslash$ Version=IA32 
L-G03RevC.02 \State $=2-A^{\prime} \backslash H F=-483.7197701 \backslash S 2=0.753695 \backslash S 2-1=0 . \backslash S 2 A=0.7500$ $09 \backslash \mathrm{RMSD}=3.459 \mathrm{e}-09 \backslash \mathrm{RMSF}=7.184 \mathrm{e}-06 \backslash \mathrm{Dipole}=0 ., 1.1367503,0.065858 \backslash \mathrm{PG}=\mathrm{CS} \quad[\mathrm{S}$ $\mathrm{G}(\mathrm{C} 1 \mathrm{H} 2 \mathrm{~N} 1 \mathrm{O} 1), \mathrm{X}(\mathrm{C} 8 \mathrm{H} 16)] \backslash \backslash @$

\section{$\cdot \mathrm{C}(\mathrm{H})\left(\mathrm{CH}_{3}\right) \mathrm{Cl}$}

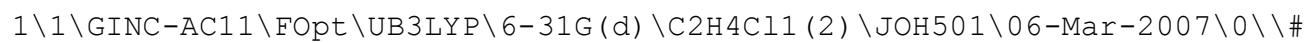

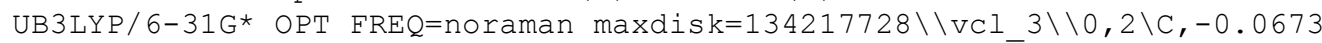
$472792,0.0192193207,-0.2424224296 \backslash \mathrm{Cl}, 1.5978066316,-0 . \overline{0} 398155931,0.2463$ $251331 \backslash \mathrm{C},-0.8419927248,1.2691524082,-0.0306703444 \backslash \mathrm{H},-0.309582796,2.143$ $2103849,-0.4247301772 \backslash \mathrm{H},-1.8096361607,1.1937414041,-0.5392674643 \backslash \mathrm{H},-1$. $0397119451,1.4648048539,1.0365562687 \backslash \mathrm{H},-0.5271157259,-0.9559027787,-0$. $3504009863 \backslash \backslash$ Version=IA64L-G03RevD.01 \State $=2-A \backslash H F=-538.7611966 \backslash S 2=0.75$ $3945 \backslash \mathrm{S} 2-1=0 . \backslash \mathrm{S} 2 \mathrm{~A}=0.750011 \backslash \mathrm{RMSD}=6.162 \mathrm{e}-09 \backslash \mathrm{RMSF}=1.212 \mathrm{e}-05 \backslash$ Therma $1=0 . \backslash \mathrm{Dip}$ $\mathrm{ole}=-0.6398134,0.1145761,-0.0270263 \backslash \mathrm{PG}=\mathrm{C} 01 \quad[\mathrm{X}(\mathrm{C} 2 \mathrm{H} 4 \mathrm{Cl} 1)] \backslash \backslash \mathrm{C}$

\section{$\cdot \mathrm{C}(\mathrm{H})\left(\mathrm{CH}_{3}\right) \mathrm{CN}$}

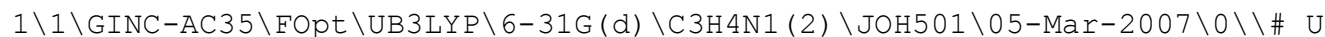

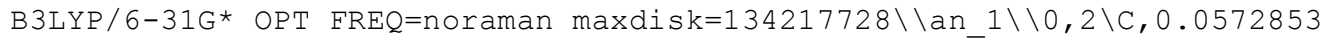
$608,0.0034909758,0 . \backslash \mathrm{C}, 1.4443410841,0.0128593863,0 . \backslash \bar{C},-0.7711955167,1.2$ $49689711,0 . \backslash \mathrm{H},-0.1519040957,2.1495862977,0 . \backslash \mathrm{H},-1.4277517503,1.27878853$ $16,-0.8809902152 \backslash \mathrm{H},-1.4277517503,1.2787885316,0.8809902152 \backslash \mathrm{H},-0.437002$ $0692,-0.9645245719,0 . \backslash N, 2.6209687382,0.0282511381,0 . \backslash \backslash$ Version=IA64L-G0 3RevD.01 \State $=2-\mathrm{A} " \backslash \mathrm{HF}=-171.4162801 \backslash \mathrm{S} 2=0.76697 \backslash \mathrm{S} 2-1=0 . \backslash \mathrm{S} 2 \mathrm{~A}=0.750126 \backslash \mathrm{RM}$ $\mathrm{SD}=6.120 \mathrm{e}-09 \backslash \mathrm{RMSF}=7.117 \mathrm{e}-05 \backslash \mathrm{Thermal}=0 . \backslash \mathrm{Dipole}=-1.5251874,0.1359253,0 . \backslash$ $\mathrm{PG}=\mathrm{CS} \quad[\mathrm{SG}(\mathrm{C} 3 \mathrm{H} 2 \mathrm{~N} 1), \mathrm{X}(\mathrm{H} 2)] \backslash \backslash \mathrm{Q}$

\section{$\cdot \mathrm{CH}_{2} \mathrm{CH}_{3}$}

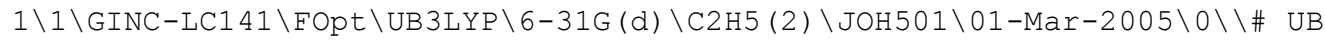
3LYP/6-31G* OPT FREQ MAXDISK=65536000 \leth 2 sym2 6 dub3 opt freq $\backslash \backslash 0,2 \backslash C$ $, 0 ., 0 ., 0 . \backslash \mathrm{C}, 0 ., 1.48959,0 . \backslash \mathrm{H},-1.02386,-0.41 \overline{6} 29,0 . \overline{\mathrm{H}}, 0.49917,-0.40758,0$. $8877 \backslash \mathrm{H}, 0.49917,-0.40758,-0.8877 \backslash \mathrm{H},-0.08019,2.0476,0.92757 \backslash \mathrm{H},-0.08019,2$ $.0476,-0.92757 \backslash \backslash$ Version $=x 86-$ Linux-G03RevB.03 State $^{2}=2-A^{\prime} \backslash H F=-79.1578672$ $\backslash \mathrm{S} 2=0.753925 \backslash \mathrm{S} 2-1=0 . \backslash \mathrm{S} 2 \mathrm{~A}=0.75001 \backslash \mathrm{RMSD}=6.578 \mathrm{e}-09 \backslash \mathrm{RMSF}=3.593 \mathrm{e}-05 \backslash \mathrm{Dipole}=$ $0.0850963,0.0505436,0 . \backslash P G=C S \quad[S G(C 2 H 1), X(H 4)] \backslash \backslash @$

\section{$\cdot \mathrm{C}(\mathrm{H})\left(\mathrm{CH}_{3}\right)_{2}$}

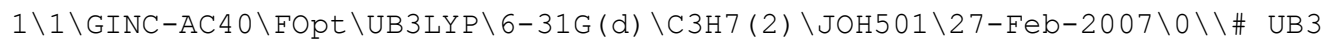
LYP/6-31G* OPT FREQ=noraman maxdisk=134217728\\chme2_2s $\backslash \backslash 0,2 \backslash \mathrm{C}, 0 ., 0 ., 0$ $. \backslash \mathrm{H}, 1.08778,0 ., 0 . \backslash \mathrm{C},-0.7109,1.30115,0.17283 \backslash \mathrm{C},-0.710 \overline{9},-1.30115,0.17283$ $\backslash \mathrm{H},-0.12833,2.14167,-0.22129 \backslash \mathrm{H},-0.12833,-2.14167,-0.22129 \backslash \mathrm{H},-1.68733,1$ $.29786,-0.33234 \backslash \mathrm{H},-1.68733,-1.29786,-0.33234 \backslash \mathrm{H},-0.91747,1.52734,1.2356$ $5 \backslash \mathrm{H},-0.91747,-1.52734,1.23565 \backslash \backslash$ Version=IA64L-G03RevD.01 $\backslash$ State=2-A $\backslash \mathrm{HF}=$ $-118.4781544 \backslash \mathrm{S} 2=0.754002 \backslash \mathrm{S} 2-1=0 . \backslash \mathrm{S} 2 \mathrm{~A}=0.750011 \backslash \mathrm{RMSD}=6.966 \mathrm{e}-09 \backslash \mathrm{RMSF}=1.51$ $6 e-05 \backslash$ Thermal $=0 . \backslash$ Dipole $=0.0681339,-0.0420556,0 . \backslash P G=C S \quad[S G(C 1 H 1), X(C 2 H 6$ )$] \backslash(\mathbb{a}$

\section{$\cdot \mathrm{C}\left(\mathrm{CH}_{3}\right)_{3}$}

$1 \backslash 1 \backslash G I N C-A C 35 \backslash F O p t \backslash U B 3 L Y P \backslash 6-31 G(d) \backslash C 4 H 9(2) \backslash J O H 501 \backslash 31-J u l-2006 \backslash 0 \backslash \backslash \#$ UB3

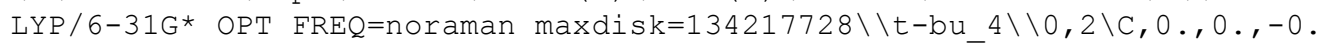
$0176048441 \backslash \mathrm{C}, 1.4891715114,0 .,-0.176787351 \backslash \mathrm{C},-0.7445 \overline{8} 57557,-1.289660359$ $5,-0.176787351 \backslash \mathrm{C},-0.7445857557,1.2896603595,-0.176787351 \backslash \mathrm{H},-0.20390525$ $32,2.130303493,0.2766588458 \backslash \mathrm{H}, 1.9468495693,-0.8885646173,0.2766588458 \backslash$ $\mathrm{H},-1.7429443161,-1.2417388758,0.2766588458 \backslash \mathrm{H},-1.7429443161,1.241738875$ $8,0.2766588458 \backslash \mathrm{H}, 1.9468495693,0.8885646173,0.2766588458 \backslash \mathrm{H},-0.203905253$ $2,-2.130303493,0.2766588458 \backslash \mathrm{H},-0.8979136693,1.5552320961,-1.2404498954$ $\backslash \mathrm{H}, 1.7958273387,0 .,-1.2404498954 \backslash \mathrm{H},-0.8979136693,-1.5552320961,-1.2404$ $498954 \backslash \backslash$ Version=IA64L-G03RevD.01 \State $=2-A 1 \backslash H F=-157.7983286 \backslash$ S2 $=0.75401$ $8 \backslash \mathrm{S} 2-1=0 . \backslash \mathrm{S} 2 \mathrm{~A}=0.750012 \backslash \mathrm{RMSD}=7.741 \mathrm{e}-09 \backslash \mathrm{RMSF}=1.499 \mathrm{e}-05 \backslash \mathrm{Thermal}=0 . \backslash \mathrm{Dipole}$ $=0 ., 0 .,-0.0761405 \backslash \mathrm{PG}=\mathrm{C} 03 \mathrm{~V} \quad[\mathrm{C} 3(\mathrm{C} 1), 3 \mathrm{SGV}(\mathrm{C} 1 \mathrm{H} 1), \mathrm{X}(\mathrm{H} 6)] \backslash \backslash \mathrm{e}$ 


\section{$\cdot \mathrm{CH}_{2} \mathrm{CH}_{2} \mathrm{CH}_{3}$}

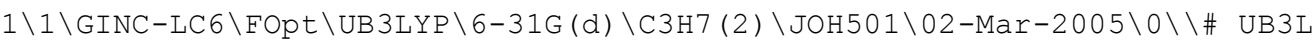

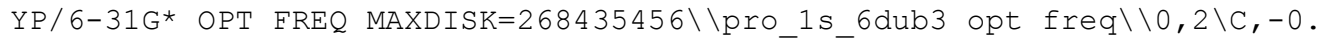
$4439980246,-1.4253596321,0 . \backslash C, 0 ., 0 ., 0 . \backslash C, \overline{-}-1 . \overline{1} 738565413,1.0088644967,0$. $\backslash \mathrm{H},-0.8009479913,2.0403994025,0 . \backslash \mathrm{H},-1.8057671583,0.8752234031,0.88507 \backslash$ $\mathrm{H},-1.8057671583,0.8752234031,-0.88507 \backslash \mathrm{H}, 0.6270126892,0.1969360169,0.88$ $052 \backslash \mathrm{H}, 0.6270126892,0.1969360169,-0.88052 \backslash \mathrm{H},-0.7036749827,-1.9279659373$ $, 0.92736 \backslash \mathrm{H},-0.7036749827,-1.9279659373,-0.92736 \backslash \backslash$ Version=x86-Linux-G03 RevB.03 $\backslash$ State $=2-A^{\prime} \backslash H F=-118.4711109 \backslash S 2=0.753855 \backslash S 2-1=0 . \backslash S 2 A=0.750009 \backslash R M$ $\mathrm{SD}=4.635 \mathrm{e}-09 \backslash \mathrm{RMSF}=2.874 \mathrm{e}-05 \backslash \mathrm{Dipole}=-0.0789651,0.0458197,0 . \backslash \mathrm{PG}=\mathrm{CS} \quad[\mathrm{SG}(\mathrm{C}$ $3 \mathrm{H} 1), \mathrm{X}(\mathrm{H} 6)] \backslash \backslash \mathrm{Q}$

\section{$\cdot \mathrm{CH}_{2}\left(\mathrm{C}_{6} \mathrm{C}_{5}\right)$}

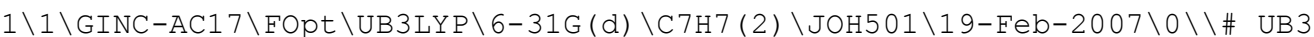

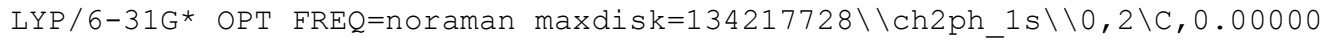
$00117,0 ., 0 . \backslash \mathrm{C}, 0.0000000117,0 ., 1.40687 \backslash \mathrm{C}, 0.0000000117,0 .,-2.83492 \backslash \mathrm{H}, 0.0$ $000000117,0 .,-3.92119 \backslash \mathrm{H}, 0.0000000117,-0.9280274624,1.9694437125 \backslash \mathrm{H}, 0.00$ $00000117,0.9280274624,1.9694437125 \backslash C, 0.0000000117,-1.2180774854,-0.742$ $9400194 \backslash \mathrm{C}, 0.0000000117,1.2180774854,-0.7429400194 \backslash \mathrm{C}, 0.0000000117,-1.21$ $20641953,-2.1288369739 \backslash \mathrm{C}, 0.0000000117,1.2120641953,-2.1288369739 \backslash \mathrm{H}, 0.0$ $000000117,-2.1606878954,-0.2009325066 \backslash \mathrm{H}, 0.0000000117,2.1606878954,-0.2$ $009325066 \backslash \mathrm{H}, 0.0000000117,-2.1540130532,-2.6714927354 \backslash \mathrm{H}, 0.0000000117,2$. $1540130532,-2.6714927354 \backslash \backslash$ Version=IA64L-G03RevD. $01 \backslash$ State $=2-A^{\prime} \backslash H F=-270$. $9151433 \backslash \mathrm{S} 2=0.783658 \backslash \mathrm{S} 2-1=0 . \backslash \mathrm{S} 2 \mathrm{~A}=0.750768 \backslash \mathrm{RMSD}=7.458 \mathrm{e}-09 \backslash \mathrm{RMSF}=7.911 \mathrm{e}-05$ $\backslash$ Thermal=0.\Dipole=0., $-0.0525407,0 . \backslash \mathrm{PG}=\mathrm{C} 02 \mathrm{~V} \quad[\mathrm{C} 2(\mathrm{H} 1 \mathrm{C} 1 \mathrm{C} 1 \mathrm{C} 1), \mathrm{SGV}(\mathrm{C} 4 \mathrm{H} 6)] \backslash \backslash$ e

\section{$\cdot \mathrm{CH}_{2} \mathrm{OH}$}

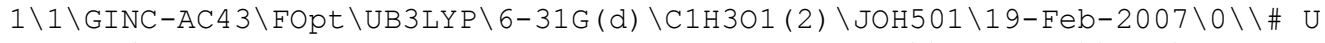
B3LYP/6-31G* OPT FREQ=noraman maxdisk=134217728 \ch2oh $1 \backslash \backslash 0,2 \backslash H, 0.0539$ $098849,0.0051141709,0.0341784915 \backslash 0,1.0099238576,0.1134 \overline{7} 39506,-0.081337$ $8927 \backslash \mathrm{C}, 1.3384431149,1.4237021764,0.1475501815 \backslash \mathrm{H}, 2.3873859182,1.6279061$ $624,-0.0333764464 \backslash \mathrm{H}, 0.5955772244,2.1904235397,-0.0670260986 \backslash \backslash$ Version=I A 64 L-G0 3RevD.01 \State $=2-A \backslash H F=-115.0520326 \backslash S 2=0.752927 \backslash \mathrm{S} 2-1=0 . \backslash \mathrm{S} 2 \mathrm{~A}=0.75$ $0006 \backslash \mathrm{RMSD}=5.600 \mathrm{e}-09 \backslash \mathrm{RMSF}=2.167 \mathrm{e}-05 \backslash \mathrm{Thermal}=0 . \backslash \mathrm{Dipole}=-0.5715567,0.1981$ $962,-0.0613129 \backslash \mathrm{PG}=\mathrm{C} 01 \quad[\mathrm{X}(\mathrm{C} 1 \mathrm{H} 301)] \backslash \backslash \mathrm{Q}$

\section{$\cdot \mathrm{CH}_{2} \mathrm{CN}$}

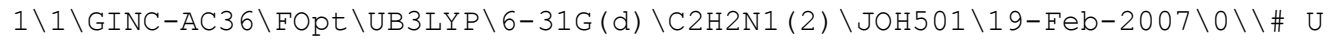
B3LYP/6-31G* OPT $F R E Q=$ noraman maxdisk $=134217728 \backslash \backslash \mathrm{ch} 2 \mathrm{cn} 1 \mathrm{~s} \backslash \backslash 0,2 \backslash \mathrm{C}, 0 ., 0$. $, 0 . \backslash \mathrm{C}, 0 ., 0 ., 1.38251 \backslash \mathrm{N}, 0 ., 0 .,-1.17677 \backslash \mathrm{H}, 0 ., 0.9358236303,1.9297030341 \backslash \mathrm{H}$, 0 ., $-0.9358236303,1.9297030341 \backslash \backslash$ Version=IA64L-G03RevD.01 $\backslash$ State=2-B1 \HF= $-132.0946681 \backslash \mathrm{S} 2=0.768747 \backslash \mathrm{S} 2-1=0 . \backslash \mathrm{S} 2 \mathrm{~A}=0.750144 \backslash \mathrm{RMSD}=3.682 \mathrm{e}-09 \backslash \mathrm{RMSF}=4.65$ $4 \mathrm{e}-05 \backslash$ Thermal $=0 . \backslash$ Dipole $=0 ., 0 .,-1.3178343 \backslash \mathrm{PG}=\mathrm{C} 02 \mathrm{~V} \quad[\mathrm{C} 2(\mathrm{C} 1 \mathrm{C} 1 \mathrm{~N} 1), \mathrm{SGV}(\mathrm{H} 2)] \backslash$ $\backslash$ @

\section{$\cdot \mathrm{CH}_{2} \mathbf{F}$}

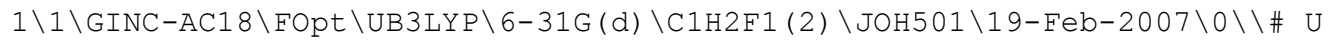
B3LYP/6-31G* OPT FREQ=noraman maxdisk=134217728\\ch2f_2 $2 \backslash 0,2 \backslash F, 0.00580$ $4342,0.0021311747,0.0229855352 \backslash C, 1.3438931985,-0.0078 \overline{2} 45133,-0.0885212$ $018 \backslash \mathrm{H}, 1.8197457765,0.959969899,0.0336036425 \backslash \mathrm{H}, 1.819466683,-0.939826926$ $1,0.1998820561 \backslash \backslash$ Version=IA64L-G03RevD.01 \State $=2-A \backslash H F=-139.0642659 \backslash \mathrm{S} 2=$ $0.753038 \backslash \mathrm{S} 2-1=0 . \backslash \mathrm{S} 2 \mathrm{~A}=0.750006 \backslash \mathrm{RMSD}=8.367 \mathrm{e}-09 \backslash \mathrm{RMSF}=1.637 \mathrm{e}-05 \backslash$ Therma $\mathrm{l}=0$. $\backslash$ Dipole $=0.4749973,0.0104757,0.1204848 \backslash \mathrm{PG}=\mathrm{C} 01 \quad[\mathrm{X}(\mathrm{C} 1 \mathrm{H} 2 \mathrm{~F} 1)] \backslash \backslash @$ 


\section{TEMPO-CH}

$1 \backslash 1 \backslash G I N C-A C 46 \backslash F O p t \backslash U B 3 L Y P \backslash 6-31 G(d) \backslash C 10 H 21 N 101 \backslash J O H 501 \backslash 27-A p r-2006 \backslash 0 \backslash \backslash \#$

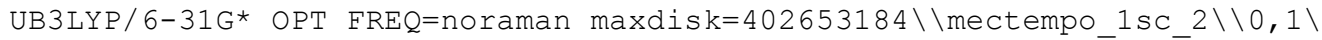
$\mathrm{N}, 0.0002988286,0.0002439874,0 . \backslash 0,1.2737059142,0.6883183539,0 . \backslash \overline{\mathrm{C}}, 2.3506$ $876745,-0.2411398131,0 . \backslash \mathrm{C},-2.8852271085,-0.0017544587,0 . \backslash \mathrm{H},-3.83338298$ $96,-0.5541032208,0 . \backslash \mathrm{H},-3.1525735473,1.0627004942,0 . \backslash \mathrm{H}, 3.2570855991,0.3$ $738897387,0 . \backslash \mathrm{H}, 2.3451131472,-0.8780068873,-0.8913880676 \backslash \mathrm{H}, 2.3451131472$ $,-0.8780068873,0.8913880676 \backslash \mathrm{C},-0.8020811717,1.8269541872,-1.6192712668$ $\backslash \mathrm{C},-0.8020811717,1.8269541872,1.6192712668 \backslash \mathrm{C},-0.6850577871,0.319741142$ $9,-1.2916421285 \backslash C,-0.6850577871,0.3197411429,1.2916421285 \backslash C, 0.12994426$ $37,-0.3436453112,-2.4192770086 \backslash C, 0.1299442637,-0.3436453112,2.41927700$ $86 \backslash C,-2.075688509,-0.3545016231,-1.24880994 \backslash C,-2.075688509,-0.35450162$ $31,1.24880994 \backslash \mathrm{H},-1.9330723624,-1.443302447,-1.2797272846 \backslash \mathrm{H},-1.93307236$ $24,-1.443302447,1.2797272846 \backslash \mathrm{H},-2.6196523776,-0.0788674,-2.1611714295 \backslash$ $\mathrm{H},-2.6196523776,-0.0788674,2.1611714295 \backslash \mathrm{H},-0.4243351909,-0.2839997465$, $-3.3631944188 \backslash \mathrm{H},-0.4243351909,-0.2839997465,3.3631944188 \backslash \mathrm{H}, 0.313614339$ $6,-1.3990122926,-2.1929785899 \backslash \mathrm{H}, 0.3136143396,-1.3990122926,2.192978589$ $9 \backslash \mathrm{H}, 1.0911307072,0.1578999551,-2.5653486667 \backslash \mathrm{H}, 1.0911307072,0.157899955$ $1,2.5653486667 \backslash \mathrm{H},-1.0670442347,1.9535642908,-2.6756148675 \backslash \mathrm{H},-1.0670442$ $347,1.9535642908,2.6756148675 \backslash \mathrm{H},-1.5667249108,2.3373165777,-1.02971442$ $56 \backslash \mathrm{H},-1.5667249108,2.3373165777,1.0297144256 \backslash \mathrm{H}, 0.1558469011,2.32426701$ $31,-1.4474030607 \backslash \mathrm{H}, 0.1558469011,2.3242670131,1.4474030607 \backslash \backslash$ Version=IA6 $4 \mathrm{~L}-\mathrm{G} 03 \mathrm{RevD} .01 \backslash \mathrm{State}=1-\mathrm{A}^{\prime} \backslash \mathrm{HF}=-523.6320317 \backslash \mathrm{S} 2=0 . \backslash \mathrm{S} 2-1=0 . \backslash \mathrm{S} 2 \mathrm{~A}=0 . \backslash \mathrm{RMSD}=7.8$ $56 \mathrm{e}-09 \backslash \mathrm{RMSF}=4.716 \mathrm{e}-06 \backslash \mathrm{Thermal}=0 . \backslash \mathrm{Dipole}=-0.127016,-0.1853998,0 . \backslash \mathrm{PG}=\mathrm{CS}$ $[\mathrm{SG}(\mathrm{C} 2 \mathrm{H} 3 \mathrm{~N} 1 \mathrm{O} 1), \mathrm{X}(\mathrm{C} 8 \mathrm{H} 18)] \backslash \backslash @$

\section{$\left(\mathrm{CH}_{3}\right)_{2} \mathrm{NO}-\mathrm{CH}_{3}$}

$1 \backslash 1 \backslash G I N C-A C 22 \backslash F O p t \backslash U B 3 L Y P \backslash 6-31 G(d) \backslash C 3 H 9 N 101 \backslash J O H 501 \backslash 19-J u l-2006 \backslash 0 \backslash \backslash \#$ UB $3 \mathrm{LYP} / 6-31 \mathrm{G} * \mathrm{OPT} \mathrm{FREQ}=$ noraman maxdisk=134217728 \\me2nome $1 \backslash \backslash 0,1 \backslash \mathrm{N}, 1.188$ $887377,-0.186152308,0 . \backslash 0,-0.0035949143,0.6399280704,0 . \backslash \bar{C},-1.1403381769$ $,-0.2073001874,0 . \backslash \mathrm{H},-2.0067642555,0.4619759379,0 . \backslash \mathrm{H},-1.1731254316,-0.8$ $464472919,0.8927583809 \backslash \mathrm{H},-1.1731254316,-0.8464472919,-0.8927583809 \backslash \mathrm{C}, 1$ $.918796164,0.1813241521,-1.209484138 \backslash C, 1.918796164,0.1813241521,1.2094$ $84138 \backslash \mathrm{H}, 2.8411095209,-0.408380338,1.2499352376 \backslash \mathrm{H}, 2.1743042081,1.253358$ $3358,1.2437296164 \backslash \mathrm{H}, 1.3091955235,-0.0655706143,2.0827280969 \backslash \mathrm{H}, 2.841109$ $5209,-0.408380338,-1.2499352376 \backslash \mathrm{H}, 1.3091955235,-0.0655706143,-2.082728$ $0969 \backslash \mathrm{H}, 2.1743042081,1.2533583358,-1.2437296164 \backslash \backslash$ Version=IA64 L-G03RevD. $01 \backslash$ State $=1-A^{\prime} \backslash H F=-249.6422563 \backslash S 2=0 . \backslash S 2-1=0 . \backslash S 2 A=0 . \backslash R M S D=2.744 e-09 \backslash R M S F$ $=3.495 e-05 \backslash$ Thermal $=0 . \backslash$ Dipole $=0.147863,-0.1723613,0 . \backslash P G=C S \quad[S G(C 1 H 1 N 101$ )$, \mathrm{X}(\mathrm{C} 2 \mathrm{H} 8)] \backslash \backslash \mathrm{C}$

\section{$\mathrm{H}_{2} \mathrm{NO}-\mathrm{CH}_{3}$}

$1 \backslash 1 \backslash G I N C-L C 14 \backslash F O p t \backslash U B 3 L Y P \backslash 6-31 G(d) \backslash C 1 H 5 N 101 \backslash J O H 501 \backslash 04-J u l-2006 \backslash 0 \backslash \backslash \#$ UB $3 \mathrm{LYP} / 6-31 \mathrm{G} *$ OPT FREQ=NORAMAN MAXDISK=39321600 \h2nome_2 $2 \backslash 0,1 \backslash \mathrm{N},-1.1191$ $595214,-0.3993931332,0 . \backslash 0,0.3222425425,-0.4802357875, \overline{0} . \backslash \mathrm{C}, 0.8394343463$ $, 0.8400176582,0 . \backslash \mathrm{H}, 1.9282633962,0.7264378919,0 . \backslash \mathrm{H}, 0.5280438178,1.39910$ $69482,0.8927023981 \backslash \mathrm{H}, 0.5280438178,1.3991069482,-0.8927023981 \backslash \mathrm{H},-1.3823$ $903998,-0.9635597523,-0.811617449 \backslash \mathrm{H},-1.3823903998,-0.9635597523,0.8116$ $17449 \backslash \backslash$ Version=IA32L-G03RevC.02 S State $=1-A \cdot \backslash H F=-171.014642 \backslash S 2=0 . \backslash S 2-1=0$ $. \backslash \mathrm{S} 2 \mathrm{~A}=0 . \backslash \mathrm{RMSD}=4.822 \mathrm{e}-09 \backslash \mathrm{RMSF}=1.262 \mathrm{e}-04 \backslash \mathrm{Dipole}=-0.1740601,-0.1433837,0$. $\backslash \mathrm{PG}=\mathrm{CS} \quad[\mathrm{SG}(\mathrm{C} 1 \mathrm{H} 1 \mathrm{~N} 1 \mathrm{O} 1), \mathrm{X}(\mathrm{H} 4)] \backslash \backslash \mathrm{e}$

\section{TEMPO-C $(\mathrm{H})\left(\mathrm{CH}_{3}\right) \mathrm{Cl}$}

$1 \backslash 1 \backslash G I N C-A C 3 \backslash F O p t \backslash U B 3 L Y P \backslash 6-31 G(d) \backslash C 11 H 22 C 11 N 101 \backslash J O H 501 \backslash 06-M a r-2007 \backslash 0 \backslash \backslash$ \# UB3LYP/6-31G* OPT FREQ=noraman maxdisk=402653184 \\tempovcl $1 \backslash \backslash 0,1 \backslash \mathrm{N}$, $-0.1957971342,0.0806466301,-0.0208804114 \backslash 0,1.2400201239,0.31 \overline{4} 0437577,0$ $.1379894307 \backslash C, 1.5880691996,1.6490140696,0.2494773774 \backslash C,-2.4562506619,-$ $1.734984466,-0.0126193158 \backslash \mathrm{H},-3.5330703354,-1.9055403157,-0.1351448927 \backslash$ $\mathrm{H},-2.0178472677,-2.7110966251,0.2294881954 \backslash \mathrm{Cl}, 2.0257168219,2.448974705$ $4,-1.3959037959 \backslash \mathrm{C}, 2.8213622834,1.753095262,1.128390561 \backslash \mathrm{C}, 0.0531175305$, 
$-1.5324619109,1.9770720129 \backslash C, 0.5058493254,-2.1143813142,-1.1737022619 \backslash$ $C,-0.7227020996,-0.3706683537,1.3158985742 \backslash C,-0.359443189,-0.832684711$ $4,-1.2067664456 \backslash \mathrm{C},-0.6821051907,0.8317002538,2.2815017422 \backslash \mathrm{C}, 0.01793449$ $6,-0.040472126,-2.4696118425 \backslash C,-2.21297724,-0.7354166128,1.1163944742 \backslash$ $\mathrm{C},-1.8639277659,-1.1790369993,-1.3059203194 \backslash \mathrm{H},-2.7675116683,0.18643341$ $09,0.8950345473 \backslash \mathrm{H},-2.409686048,-0.267142065,-1.5826348962 \backslash \mathrm{H},-2.5968203$ $98,-1.1155420054,2.0713220183 \backslash \mathrm{H},-1.9891310021,-1.887528838,-2.13377633$ $54 \backslash \mathrm{H},-1.2456215157,0.5865693382,3.1888397511 \backslash \mathrm{H},-0.2914398736,-0.606058$ $8139,-3.3565000847 \backslash \mathrm{H},-1.1401129931,1.7154078766,1.8255773128 \backslash \mathrm{H},-0.4828$ $425745,0.9316211777,-2.4827528807 \backslash \mathrm{H}, 0.3368114125,1.0784841591,2.592916$ $2452 \backslash \mathrm{H}, 1.0936439991,0.1309156162,-2.5331966879 \backslash \mathrm{H},-0.2474626914,-1.6179$ $930045,3.0277843617 \backslash \mathrm{H}, 0.5134248829,-2.5665133765,-2.1720085171 \backslash \mathrm{H},-0.13$ $55255292,-2.4982991641,1.5056267891 \backslash \mathrm{H}, 0.1369924085,-2.871784808,-0.478$ $5770172 \backslash \mathrm{H}, 1.1290305904,-1.3422603811,1.9483281993 \backslash \mathrm{H}, 1.5380333307,-1.87$ $3724356,-0.907740636 \backslash \mathrm{H}, 3.1553898884,2.7904793973,1.2000159747 \backslash \mathrm{H}, 2.5900$ $736222,1.3787672094,2.1316761253 \backslash \mathrm{H}, 3.6307515322,1.1490779874,0.7094153$ $272 \backslash \mathrm{H}, 0.7470737306,2.2517093966,0.5817073202 \backslash \backslash$ Version=IA64 L-G0 3RevD. 01 $\backslash$ State $=1-\mathrm{A} \backslash \mathrm{HF}=-1022.5502512 \backslash \mathrm{S} 2=0 . \backslash \mathrm{S} 2-1=0 . \backslash \mathrm{S} 2 \mathrm{~A}=0 . \backslash \mathrm{RMSD}=6.620 \mathrm{e}-09 \backslash \mathrm{RMSF}=1$ $.056 \mathrm{e}-05 \backslash \mathrm{Thermal}=0 . \backslash \mathrm{Dipole}=-0.5919315,-0.6119132,0.9158416 \backslash \mathrm{PG}=\mathrm{C} 01 \quad[\mathrm{X}(\mathrm{C}$ $11 \mathrm{H} 22 \mathrm{Cl} 1 \mathrm{~N} 1 \mathrm{O} 1)] \backslash \backslash \mathrm{e}$

\section{$\left(\mathrm{CH}_{3}\right)_{2} \mathrm{NO}-\mathrm{C}(\mathrm{H})\left(\mathrm{CH}_{3}\right) \mathrm{Cl}$}

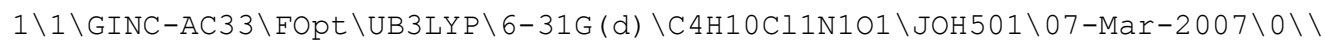

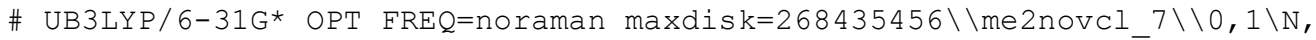
$-0.1719121943,-0.0606865797,-0.0678220263 \backslash 0,1.0523277611,0.3 \overline{1} 75196569$, $0.6334229329 \backslash \mathrm{C}, 1.4351550229,1.5986382819,0.3003477737 \backslash \mathrm{Cl}, 2.2983731082$, $1.6492422735,-1.3678450127 \backslash \mathrm{C}, 2.3980805602,2.1056335638,1.3545642738 \backslash \mathrm{C}$, $-1.1255890058,-0.3833116786,0.9909815365 \backslash \mathrm{C}, 0.1768322312,-1.2363105409$, $-0.8655472173 \backslash \mathrm{H}, 2.7564580872,3.104078874,1.0947232659 \backslash \mathrm{H}, 1.8822229303,2$ $.1467805486,2.3204133386 \backslash \mathrm{H}, 3.2543607606,1.4314884225,1.4405643753 \backslash \mathrm{H}, 0$. $5686057227,2.2415337074,0.1397505864 \backslash \mathrm{H},-2.0598290996,-0.6987070731,0.5$ $15533294 \backslash \mathrm{H},-0.7701078865,-1.1861847688,1.656368079 \backslash \mathrm{H},-1.3231773457,0.5$ $131495662,1.5846512975 \backslash \mathrm{H},-0.727019498,-1.5596671748,-1.3921783756 \backslash \mathrm{H}, 0$. $9324183615,-0.9534343566,-1.5995361888 \backslash \mathrm{H}, 0.553940484,-2.0688727223,-0$. $2506419329 \backslash \backslash$ Version=IA6 4L-G03RevD.01 \State=1-A \HF=-748.5659972\S2=0. \S $2-1=0 . \backslash \mathrm{S} 2 \mathrm{~A}=0 . \backslash \mathrm{RMSD}=7.888 \mathrm{e}-09 \backslash \mathrm{RMSF}=5.664 \mathrm{e}-05 \backslash \mathrm{Thermal}=0 . \backslash \mathrm{Dipole}=-0.71952$ $13,-0.2617612,0.7949552 \backslash \mathrm{PG}=\mathrm{C} 01 \quad[\mathrm{X}(\mathrm{C} 4 \mathrm{H} 10 \mathrm{Cl} 1 \mathrm{~N} 1 \mathrm{O} 1)] \backslash \backslash \mathrm{Q}$

\section{$\mathrm{H}_{2} \mathrm{NO}-\mathrm{C}(\mathrm{H})\left(\mathrm{CH}_{3}\right) \mathrm{Cl}$}

$1 \backslash 1 \backslash G I N C-A C 54 \backslash F O p t \backslash U B 3 L Y P \backslash 6-31 G(d) \backslash C 2 H 6 C l 1 N 101 \backslash J O H 501 \backslash 22-M a r-2007 \backslash 0 \backslash \backslash \#$ UB3LYP/6-31G* OPT FREQ=noraman maxdisk=134217728 \h2novcl_4 $\backslash \backslash 0,1 \backslash \mathrm{C},-0$ $.2830935482,-0.2289261328,-0.4621877641 \backslash 0,1.0895136386,-0 . \overline{2} 423028017,-$ $0.5441102302 \backslash \mathrm{N}, 1.5775474165,1.0992493541,-0.8017212996 \backslash \mathrm{H}, 1.7813523695$, $1.4611796455,0.1353645498 \backslash \mathrm{H}, 2.4870534239,0.8985287378,-1.2209934523 \backslash \mathrm{C}$, $-0.7915903659,-1.6511118659,-0.5522639321 \backslash \mathrm{H},-0.7161135157,0.4568674765$ $,-1.1919400688 \backslash \mathrm{Cl},-0.8304742199,0.5121536734,1.1808297176 \backslash \mathrm{H},-1.8772801$ $395,-1.6743999835,-0.4365324571 \backslash \mathrm{H},-0.5248819975,-2.0628029512,-1.53200$ $61054 \backslash \mathrm{H},-0.3364030618,-2.2672951521,0.2274310423 \backslash \backslash$ Version=IA $64 \mathrm{~L}-\mathrm{G} 03 \mathrm{ReV}$ D. 01 $\backslash$ State $=1-A \backslash H F=-669.9397019 \backslash S 2=0 . \backslash S 2-1=0 . \backslash S 2 A=0 . \backslash R M S D=3.063 e-09 \backslash R M S$ $\mathrm{F}=1.200 \mathrm{e}-05 \backslash \mathrm{Thermal}=0 . \backslash \mathrm{Dipole}=0.603508,-0.2572103,-0.6024676 \backslash \mathrm{PG}=\mathrm{C} 01 \quad[\mathrm{X}$ $(\mathrm{C} 2 \mathrm{H} 6 \mathrm{Cl} 1 \mathrm{~N} 1 \mathrm{O} 1)] \backslash \backslash \mathrm{e}$

\section{TEMPO-C(H)(CH$) \mathrm{CN}$}

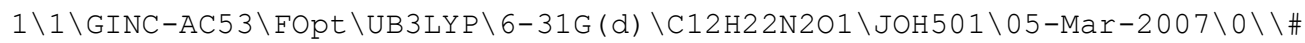

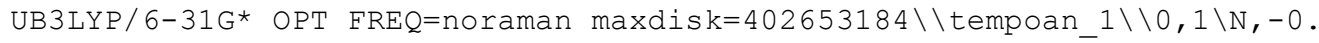
$0816853495,0.0184041994,0.0377511396 \backslash 0,1.3441802645,0.179 \overline{3} 512702,0.289$ $9100227 \backslash \mathrm{C}, 1.7576072602,1.5454029626,0.3168739367 \backslash \mathrm{C},-2.4449687332,-1.65$ $11429783,-0.0939827647 \backslash \mathrm{H},-3.5197522747,-1.7581104733,-0.2865869795 \backslash \mathrm{H},-$ $2.0833489308,-2.6494983585,0.1820304003 \backslash \mathrm{C}, 2.1819533179,2.0515427683,-1$ $.0128383326 \backslash \mathrm{C}, 2.9374145272,1.6408391435,1.2911126259 \backslash \mathrm{C},-0.0630463092,-$ $1.5794816172,2.0578612946 \backslash C, 0.557880206,-2.2229333798,-1.0552708051 \backslash C$, 
$-0.7230173005,-0.3815775695,1.337616575 \backslash C,-0.223190619,-0.8914850101,-$ $1.1510212822 \backslash \mathrm{C},-0.6638646588,0.8301956167,2.2896169131 \backslash \mathrm{C}, 0.2877736592$, $-0.1327996491,-2.3878343787 \backslash C,-2.2165230347,-0.6576913625,1.0441620026$ $\backslash \mathrm{C},-1.7361477772,-1.1454665115,-1.3493944214 \backslash \mathrm{H},-2.6989647368,0.2935499$ $516,0.7816764976 \backslash \mathrm{H},-2.2051523755,-0.204297132,-1.6663931921 \backslash \mathrm{H},-2.68497$ $86068,-1.0056133888,1.9733657639 \backslash \mathrm{H},-1.8520805058,-1.8531341649,-2.1793$ $946862 \backslash \mathrm{H},-1.2839459344,0.6329332472,3.1713224096 \backslash \mathrm{H},-0.0021492382,-0.67$ $69138824,-3.2941816141 \backslash \mathrm{H},-1.0480605119,1.7313748671,1.8002282793 \backslash \mathrm{H},-0$. $1436007849,0.8713290082,-2.4363474453 \backslash \mathrm{H}, 0.3525313014,1.0219168423,2.64$ $58156349 \backslash \mathrm{H}, 1.3757059247,-0.0409313781,-2.3902114284 \backslash \mathrm{H},-0.4308007552,-1$ $.6329612916,3.0891990527 \backslash \mathrm{H}, 0.6044920741,-2.685240212,-2.0479175871 \backslash \mathrm{H},-$ $0.2823092956,-2.5384124053,1.5850801164 \backslash \mathrm{H}, 0.0984905516,-2.9492104986,-$ $0.3811758297 \backslash \mathrm{H}, 1.0221682413,-1.4542547725,2.0920798635 \backslash \mathrm{H}, 1.5827052419$, $-2.0414932666,-0.7216999834 \backslash \mathrm{H}, 3.3257784656,2.663142728,1.3258349768 \backslash \mathrm{H}$, $2.6117063953,1.3465813848,2.2923477897 \backslash \mathrm{H}, 3.7418678466,0.9719498954,0.9$ $726073981 \backslash \mathrm{H}, 0.926345397,2.1725850376,0.6494624817 \backslash \mathrm{N}, 2.5543470581,2.486$ $8003793,-2.0224444444 \backslash \backslash$ Version=IA64L-G03RevD.01 \State=1-A \HF=-655.1836 $032 \backslash \mathrm{S} 2=0 . \backslash \mathrm{S} 2-1=0 . \backslash \mathrm{S} 2 \mathrm{~A}=0 . \backslash \mathrm{RMSD}=7.853 \mathrm{e}-09 \backslash \mathrm{RMSF}=7.567 \mathrm{e}-06 \backslash \mathrm{Thermal}=0 . \backslash \mathrm{Dipo}$ $l e=-0.745638,-0.5919885,1.2725652 \backslash \mathrm{PG}=\mathrm{C} 01 \quad[\mathrm{X}(\mathrm{C} 12 \mathrm{H} 22 \mathrm{~N} 2 \mathrm{O} 1)] \backslash \backslash \mathrm{e}$

\section{$\left(\mathrm{CH}_{3}\right)_{2} \mathrm{NO}-\mathrm{C}(\mathrm{H})\left(\mathrm{CH}_{3}\right) \mathrm{CN}$}

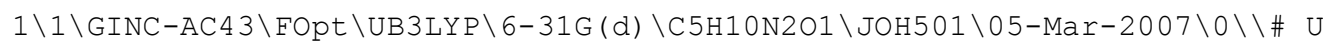
B3LYP/6-31G* OPT FREQ=noraman maxdisk=268435456\\me2noan $3 \backslash \backslash 0,1 \backslash N,-0.0$ $098868772,0.0893966424,-0.0464735048 \backslash 0,1.4456714827,0.0506951928,-0.10$ $77748091 \backslash C, 1.9582882431,1.371568955,-0.0044271362 \backslash C, 1.6271608089,2.169$ $2533766,-1.2117746156 \backslash C, 3.4720553767,1.2507658866,0.1780246402 \backslash C,-0.36$ $70254781,-0.7100269105,1.1225487859 \backslash C,-0.4767997036,-0.5198256343,-1.2$ $911102596 \backslash \mathrm{H}, 3.9260363278,2.2417200709,0.2693017039 \backslash \mathrm{H}, 3.6788612318,0.67$ $5799099,1.0848505675 \backslash \mathrm{H}, 3.9178712492,0.7356839511,-0.6771551774 \backslash \mathrm{H},-1.57$ $14174851,-0.5297246204,-1.2698290726 \backslash \mathrm{H},-0.1503739189,0.0928636176,-2.1$ $336832649 \backslash \mathrm{H},-0.1088547902,-1.5499351284,-1.4223080566 \backslash \mathrm{H},-1.4587378642$, $-0.7236816965,1.2035551324 \backslash \mathrm{H}, 0.0037441995,-1.7456056284,1.0594023259 \backslash \mathrm{H}$ $, 0.0416491507,-0.2385750239,2.020404933 \backslash \mathrm{H}, 1.5058730616,1.8784435299,0$. $8581787603 \backslash \mathrm{N}, 1.3790049853,2.7849443205,-2.1638909522 \backslash \backslash$ Version=IA $64 \mathrm{~L}-\mathrm{G} 0$ 3RevD.01 S tate=1-A \HF=-381.1982639 $\backslash \mathrm{S} 2=0 . \backslash \mathrm{S} 2-1=0 . \backslash \mathrm{S} 2 \mathrm{~A}=0 . \backslash \mathrm{RMSD}=7.033 e-09$ $\backslash \mathrm{RMSF}=1.138 \mathrm{e}-05 \backslash \mathrm{Thermal}=0 . \backslash \mathrm{Dipole}=0.045768,-0.8310612,1.1643857 \backslash \mathrm{PG}=\mathrm{C} 01$ $[\mathrm{X}(\mathrm{C} 5 \mathrm{H} 10 \mathrm{~N} 2 \mathrm{O} 1)] \backslash \backslash \mathrm{e}$

\section{$\mathrm{H}_{2} \mathrm{NO}-\mathrm{C}(\mathrm{H})\left(\mathrm{CH}_{3}\right) \mathrm{CN}$}

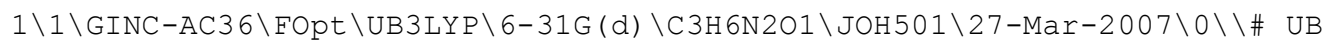

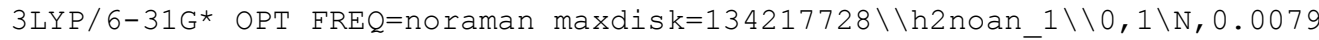
$220411,0.0291262634,0.1341480439 \backslash 0,1.4425719859,0.0067 \overline{9} 66053,-0.053573$ $3172 \backslash C, 1.9273296068,1.3434976706,-0.0492625471 \backslash C, 1.4818869223,2.060415$ $8945,-1.2705044254 \backslash \mathrm{C}, 3.4518776117,1.2654327084,0.0259643667 \backslash \mathrm{H},-0.13594$ $66404,-0.7487281389,0.7802542944 \backslash \mathrm{H},-0.3602889143,-0.2881232494,-0.7662$ $339263 \backslash \mathrm{H}, 3.8843806933,2.2699787759,0.0318624904 \backslash \mathrm{H}, 3.7390189009,0.74559$ $8157,0.94410757 \backslash \mathrm{H}, 3.8482970813,0.7158794034,-0.8321135928 \backslash \mathrm{H}, 1.51763339$ $62,1.8778155668,0.8172992012 \backslash \mathrm{N}, 1.1284373153,2.600860343,-2.2348581578 \backslash$

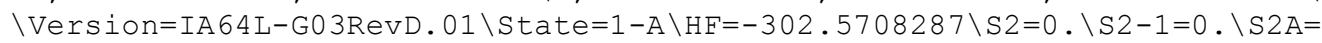
$0 . \backslash \mathrm{RMSD}=2.838 \mathrm{e}-09 \backslash \mathrm{RMSF}=1.249 \mathrm{e}-05 \backslash \mathrm{Thermal}=0 . \backslash \mathrm{Dipole}=0.2606062,-0.897773$ $4,1.0605626 \backslash P G=C 01 \quad[X(C 3 \mathrm{H} 6 \mathrm{~N} 2 \mathrm{O} 1)] \backslash \backslash @$

\section{TEMPO- $\mathrm{CH}_{2} \mathrm{CH}_{3}$}

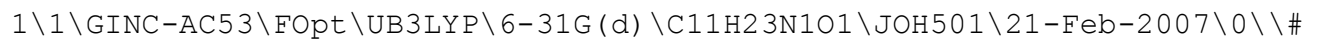

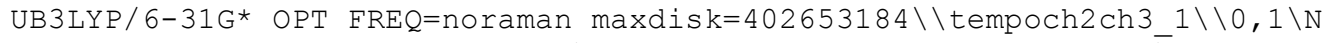
$,-0.3035380896,0.1490692731,0 . \backslash 0,0.3772395411,1.4251685459,0 . \backslash \mathrm{C},-0.550$ $4257654,2.5139041788,0 . \backslash \mathrm{C},-0.2872605697,-2.7371915564,0 . \backslash \mathrm{H},-0.83359103$ $31,-3.6888570995,0 . \backslash \mathrm{H}, 0.778592516,-2.9975612752,0 . \backslash \mathrm{C}, 0.2766148847,3.79$ $11174212,0 . \backslash \mathrm{H},-1.1957570655,2.4653456963,0.8852053866 \backslash \mathrm{H},-1.1957570655$, $2.4653456963,-0.8852053866 \backslash \mathrm{C}, 1.5284998828,-0.6438813227,1.6188259773 \backslash \mathrm{C}$ $, 1.5284998828,-0.6438813227,-1.6188259773 \backslash \mathrm{C}, 0.0204387358,-0.53499788,1$ 
$.2913446734 \backslash C, 0.0204387358,-0.53499788,-1.2913446734 \backslash C,-0.6467586562,0$ $.2749456251,2.4203217661 \backslash C,-0.6467586562,0.2749456251,-2.4203217661 \backslash C$, $-0.6454470765,-1.9299465463,1.248593889 \backslash C,-0.6454470765,-1.9299465463$, $-1.248593889 \backslash \mathrm{H},-1.7349291507,-1.7931936066,1.2788095899 \backslash \mathrm{H},-1.734929150$ $7,-1.7931936066,-1.2788095899 \backslash \mathrm{H},-0.3669706849,-2.4725012364,2.16093257$ $99 \backslash \mathrm{H},-0.3669706849,-2.4725012364,-2.1609325799 \backslash \mathrm{H},-0.5812814069,-0.2792$ $05587,3.3639631251 \backslash \mathrm{H},-0.5812814069,-0.279205587,-3.3639631251 \backslash \mathrm{H},-1.703$ $7238105,0.4516787005,2.1964585051 \backslash \mathrm{H},-1.7037238105,0.4516787005,-2.1964$ $585051 \backslash \mathrm{H},-0.1513321044,1.2394226031,2.565804201 \backslash \mathrm{H},-0.1513321044,1.2394$ $226031,-2.565804201 \backslash \mathrm{H}, 1.6559509961,-0.9084823264,2.6752086048 \backslash \mathrm{H}, 1.6559$ $509961,-0.9084823264,-2.6752086048 \backslash \mathrm{H}, 2.0438467328,-1.4054751347,1.0296$ $677123 \backslash \mathrm{H}, 2.0438467328,-1.4054751347,-1.0296677123 \backslash \mathrm{H}, 2.0208337504,0.316$ $6566434,1.4474324704 \backslash \mathrm{H}, 2.0208337504,0.3166566434,-1.4474324704 \backslash \mathrm{H},-0.38$ $10496213,4.6674354937,0 . \backslash \mathrm{H}, 0.9174989265,3.8396368799,0.886746559 \backslash \mathrm{H}, 0.9$ $174989265,3.8396368799,-0.886746559 \backslash \backslash$ Version=IA64L-G03RevD.01 \State=1$A^{\prime} \backslash H F=-562.9511382 \backslash S 2=0 . \backslash S 2-1=0 . \backslash S 2 A=0 . \backslash R M S D=8.619 e-09 \backslash R M S F=3.717 e-06 \backslash$ Thermal $=0 . \backslash$ Dipole $=-0.1597581,-0.0948486,0 . \backslash P G=C S \quad[S G(C 3 H 3 N 1 O 1), X(C 8 H 20$ )$] \backslash \backslash 0$

\section{$\left(\mathrm{CH}_{3}\right)_{2} \mathrm{NO}-\mathrm{CH}_{2} \mathrm{CH}_{3}$}

$1 \backslash 1 \backslash G I N C-A C 42 \backslash F O p t \backslash U B 3 L Y P \backslash 6-31 G(d) \backslash C 4 H 11 N 101 \backslash J O H 501 \backslash 27-M a r-2007 \backslash 0 \backslash \backslash \# \quad U$

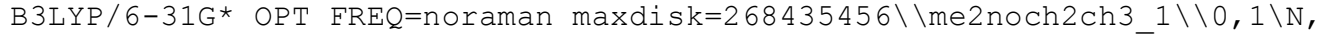
$-0.407026323,0.1631071326,0 . \backslash 0,0.3081020723,1.4231572872,0 . \backslash \bar{C},-0.63343$ $40111,2.4936142885,0 . \backslash \mathrm{C}, 0.1604698729,3.7911055256,0 . \backslash \mathrm{H},-1.2806735367,2$ $.4269333866,0.8865313908 \backslash \mathrm{H},-1.2806735367,2.4269333866,-0.8865313908 \backslash \mathrm{C}$, $0.0219036915,-0.5324423165,1.2092048368 \backslash \mathrm{C}, 0.0219036915,-0.5324423165,-$ $1.2092048368 \backslash \mathrm{H},-0.5187876563,4.6506870578,0 . \backslash \mathrm{H}, 0.7994679175,3.85443144$ $36,0.8869431122 \backslash \mathrm{H}, 0.7994679175,3.8544314436,-0.8869431122 \backslash \mathrm{H},-0.4834068$ $415,-1.503642357,1.2484336922 \backslash \mathrm{H}, 1.1124021985,-0.6921026386,1.244876613$ $5 \backslash \mathrm{H},-0.2789704067,0.0518168361,2.0828982872 \backslash \mathrm{H},-0.4834068415,-1.5036423$ $57,-1.2484336922 \backslash \mathrm{H},-0.2789704067,0.0518168361,-2.0828982872 \backslash \mathrm{H}, 1.112402$ $1985,-0.6921026386,-1.2448766135 \backslash \backslash$ Version=IA64L-G03RevD.01 \State=1-A ' $\mathrm{HF}=-288.9613101 \backslash \mathrm{S} 2=0 . \backslash \mathrm{S} 2-1=0 . \backslash \mathrm{S} 2 \mathrm{~A}=0 . \backslash \mathrm{RMSD}=4.937 \mathrm{e}-09 \backslash \mathrm{RMSF}=1.560 \mathrm{e}-05 \backslash \mathrm{The}$ $r m a l=0 . \backslash$ Dipole $=-0.1255133,-0.1265696,0 . \backslash P G=C S \quad[S G(C 2 H 1 N 1 O 1), X(C 2 H 10)] \backslash$ $\backslash$ @

\section{$\mathrm{H}_{2} \mathrm{NO}-\mathrm{CH}_{2} \mathrm{CH}_{3}$}

$1 \backslash 1 \backslash G I N C-A C 6 \backslash F O p t \backslash U B 3 L Y P \backslash 6-31 G(d) \backslash C 2 H 7 N 101 \backslash J O H 501 \backslash 27-M a r-2007 \backslash 0 \backslash \backslash \#$ UB3 LYP/6-31G* OPT FREQ=noraman maxdisk=268435456\\h2noch2ch3 $1 \backslash \backslash 0,1 \backslash \mathrm{N},-0$. $0133433933,0.0922925175,0 . \backslash 0,1.4271254414,0.0055791748,0 . \backslash \mathrm{C}, 1.96259631$ $03,1.3268745198,0 . \backslash \mathrm{C}, 3.4774836849,1.1935793826,0 . \backslash \mathrm{H}, 1.6123249926,1.875$ $2894,0.8864874672 \backslash \mathrm{H}, 1.6123249926,1.8752894,-0.8864874672 \backslash \mathrm{H},-0.27976023$ $23,-0.4707297606,0.8114484946 \backslash \mathrm{H},-0.2797602323,-0.4707297606,-0.8114484$ $946 \backslash \mathrm{H}, 3.9441519288,2.1847033546,0 . \backslash \mathrm{H}, 3.8190732538,0.650015885,0.887095$ $3728 \backslash \mathrm{H}, 3.8190732538,0.650015885,-0.8870953728 \backslash \backslash$ Version=IA64L-G03RevD.0 $1 \backslash$ tate $=1-A^{\prime} \backslash H F=-210.333734 \backslash S 2=0 . \backslash S 2-1=0 . \backslash S 2 A=0 . \backslash R M S D=7.058 e-09 \backslash R M S F=6$ $.006 e-05 \backslash$ Thermal $=0 . \backslash$ Dipole $=-0.132531,-0.1596223,0 . \backslash P G=C S \quad[S G(C 2 H 1 N 1 O 1)$ $, \mathrm{X}(\mathrm{H} 6)] \backslash \backslash \mathrm{Q}$

\section{TEMPO-C $(\mathrm{H})\left(\mathrm{CH}_{3}\right)_{2}$}

$1 \backslash 1 \backslash G I N C-A C 58 \backslash F O p t \backslash U B 3 L Y P \backslash 6-31 G(d) \backslash C 12 H 25 N 101 \backslash J O H 501 \backslash 27-F e b-2007 \backslash 0 \backslash \backslash \#$ UB3LYP/6-31G* OPT FREQ=noraman maxdisk=402653184 \tempochme2 $1 \backslash \backslash 0,1 \backslash \mathrm{N}$, $-0.1555658805,0.022616255,-0.0821555297 \backslash 0,0.9714361085,-0.11 \overline{7} 6331712,0$ $.8160751398 \backslash \mathrm{C}, 2.2495924666,-0.3471097207,0.1793273532 \backslash \mathrm{C},-3.0235312743$, $0.174506503,-0.5299601871 \backslash \mathrm{H},-3.8669966236,0.3117588595,-1.2183740656 \backslash \mathrm{H}$ $,-3.4623018792,0.0164643035,0.4631331923 \backslash C, 3.2400935884,-0.3234685005$, $1.3409680051 \backslash \mathrm{C}, 2.6151004401,0.6454177299,-0.9208268219 \backslash \mathrm{C},-1.2293517673$ $, 1.3446271629,1.8480593746 \backslash C,-1.3046826552,-1.8185027146,1.3095756715 \backslash$ $C,-0.8701823092,1.2760453596,0.343519138 \backslash C,-0.9344192923,-1.2649828029$ $,-0.0863003665 \backslash \mathrm{C}, 0.0420088939,2.4771286414,0.0349221827 \backslash \mathrm{C},-0.086060603$ $,-2.3392755575,-0.7979375708 \backslash C,-2.133455108,1.4163566022,-0.5361497843$ 
$\backslash \mathrm{C},-2.1955146701,-1.0372258113,-0.9535477159 \backslash \mathrm{H},-1.8158341701,1.6164040$ $247,-1.5686092466 \backslash \mathrm{H},-1.8758150614,-0.8993028918,-1.9955082033 \backslash \mathrm{H},-2.688$ $9137724,2.3001371332,-0.1976611774 \backslash \mathrm{H},-2.7970284694,-1.9546009903,-0.92$ $47717026 \backslash \mathrm{H},-0.5096297264,3.4081241227,0.2095545654 \backslash \mathrm{H},-0.7212373322,-3$. $1962635279,-1.0515631235 \backslash \mathrm{H}, 0.3686939898,2.4640132415,-1.0086144314 \backslash \mathrm{H}, 0$ $.3463450698,-1.9461393823,-1.7235814447 \backslash \mathrm{H}, 0.9241943545,2.4820723305,0$. $6794689767 \backslash \mathrm{H}, 0.7201685675,-2.7117817516,-0.159784608 \backslash \mathrm{H},-1.5039570313,2$ $.373247055,2.1097172893 \backslash \mathrm{H},-1.5838054182,-2.8752355425,1.2225682468 \backslash \mathrm{H},-$ $2.0712511254,0.7061791378,2.1225775908 \backslash \mathrm{H},-2.1462904011,-1.2993716292,1$ $.7722473884 \backslash \mathrm{H},-0.3672499062,1.0588775343,2.4553201093 \backslash \mathrm{H},-0.4443037161$, $-1.748431262,1.9798535639 \backslash \mathrm{H}, 3.5483945924,0.3234556202,-1.3981396853 \backslash \mathrm{H}$, $1.832804991,0.6812977714,-1.6826435412 \backslash \mathrm{H}, 2.7671089681,1.6542517994,-0$. $5240365637 \backslash \mathrm{H}, 4.2477519002,-0.5671656465,0.9868844722 \backslash \mathrm{H}, 3.2656106124,0$. $6700044182,1.8026057935 \backslash \mathrm{H}, 2.9563027768,-1.0509940402,2.1081924926 \backslash \mathrm{H}, 2$. $2484108728,-1.3513406627,-0.2605147764 \backslash \backslash$ Version=IA64L-G03RevD.01 \State $=1-\mathrm{A} \backslash \mathrm{HF}=-602.2633236 \backslash \mathrm{S} 2=0 . \backslash \mathrm{S} 2-1=0 . \backslash \mathrm{S} 2 \mathrm{~A}=0 . \backslash \mathrm{RMSD}=5.854 \mathrm{e}-09 \backslash \mathrm{RMSF}=6.690 \mathrm{e}-0$ $6 \backslash$ Thermal $=0 . \backslash$ Dipole $=-0.0802075,-0.0836857,-0.179901 \backslash P G=C 01 \quad[X(C 12 \mathrm{H} 25 \mathrm{~N} 1$ $01)] \backslash \backslash @$

\section{$\left(\mathrm{CH}_{3}\right)_{2} \mathrm{NO}-\mathrm{C}(\mathrm{H})\left(\mathrm{CH}_{3}\right)_{2}$}

$1 \backslash 1 \backslash G I N C-A C 7 \backslash F O p t \backslash U B 3 L Y P \backslash 6-31 G(d) \backslash C 5 H 13 N 101 \backslash J O H 501 \backslash 27-M a r-2007 \backslash 0 \backslash \backslash \#$ UB

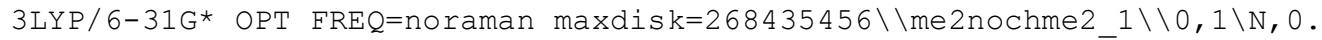
$0062328325,0.0369365901,0.183512354 \backslash 0,1.4480827081,0.0020378226,0.3216$ $741034 \backslash C, 2.0015421462,1.3066584191,0.0837960951 \backslash C, 3.5060710145,1.08432$ $37083,-0.0379329922 \backslash C, 1.6324887372,2.2892642764,1.1935586051 \backslash C,-0.5149$ $761436,-0.6173123051,1.379303006 \backslash \mathrm{C},-0.2949922503,-0.7296146583,-1.0231$ $53506 \backslash \mathrm{H}, 2.0438462278,3.2835309934,0.9823332215 \backslash \mathrm{H}, 0.5452623934,2.378143$ $7415,1.2734184969 \backslash \mathrm{H}, 2.0318521163,1.9487574522,2.1563928071 \backslash \mathrm{H}, 1.6076839$ $972,1.6813006371,-0.8731753032 \backslash \mathrm{H}, 4.015900777,2.029250743,-0.255022024 \backslash$ $\mathrm{H}, 3.9104750334,0.682134871,0.8978167211 \backslash \mathrm{H}, 3.728794706,0.3741403338,-0$. $8405340581 \backslash \mathrm{H},-1.6074589837,-0.6506291905,1.3062046109 \backslash \mathrm{H},-0.1324526042$, $-1.6442856757,1.5021260964 \backslash \mathrm{H},-0.2386870973,-0.0300673721,2.2581669629 \backslash$ $\mathrm{H},-1.3813277076,-0.7435730488,-1.1643260584 \backslash \mathrm{H}, 0.1588489446,-0.23326428$ $25,-1.8853288808 \backslash \mathrm{H}, 0.0769431526,-1.7660830555,-0.9715602576 \backslash \backslash$ Version=I A64L-G03RevD.01 \State=1-A \HF=-328.279615 \S2=0. \S2-1=0. \S2A=0. RMSD=3.3 $57 e-09 \backslash \operatorname{RMSF}=1.409 e-05 \backslash \mathrm{Thermal}=0 . \backslash \mathrm{Dipole}=-0.1927979,0.0389412,-0.099917$ $6 \backslash \mathrm{PG}=\mathrm{C} 01 \quad[\mathrm{X}(\mathrm{C} 5 \mathrm{H} 13 \mathrm{~N} 1 \mathrm{O} 1)] \backslash \backslash \mathrm{Q}$

\section{$\mathrm{H}_{2} \mathrm{NO}-\mathrm{C}(\mathrm{H})\left(\mathrm{CH}_{3}\right)_{2}$}

$1 \backslash 1 \backslash G I N C-A C 26 \backslash F O p t \backslash U B 3 L Y P \backslash 6-31 G(d) \backslash C 3 H 9 N 101 \backslash J O H 501 \backslash 22-M a r-2007 \backslash 0 \backslash \backslash \#$ UB

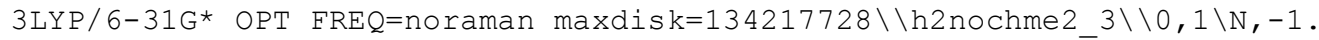
$67079,-0.89061,-0.36057 \backslash 0,-0.23796,-0.93294,-0.19153 \backslash \mathrm{C}, 0 . \overline{2} 8729,0.40462$ $,-0.2133 \backslash \mathrm{C}, 1.79885,0.23491,-0.32751 \backslash \mathrm{C},-0.13297,1.18879,1.02819 \backslash \mathrm{H},-2.01$ $905,-1.41252,0.44665 \backslash \mathrm{H},-1.82421,-1.50709,-1.16196 \backslash \mathrm{H}, 0.25317,2.21427,0$. $98995 \backslash \mathrm{H},-1.22398,1.23662,1.09398 \backslash \mathrm{H}, 0.25428,0.70789,1.93464 \backslash \mathrm{H},-0.09945$, $0.91141,-1.1095 \backslash \mathrm{H}, 2.28972,1.21263,-0.38144 \backslash \mathrm{H}, 2.1919,-0.29965,0.54476 \backslash \mathrm{H}$ $, 2.05779,-0.3358,-1.22502 \backslash \backslash$ Version=IA64L-G03RevD.01 State=1-A $\backslash H F=-249$. $6525153 \backslash \mathrm{S} 2=0 . \backslash \mathrm{S} 2-1=0 . \backslash \mathrm{S} 2 \mathrm{~A}=0 . \backslash \mathrm{RMSD}=6.540 \mathrm{e}-09 \backslash \mathrm{RMSF}=2.307 \mathrm{e}-05 \backslash \mathrm{Thermal}=0 . \backslash$ Dipole $=-0.2253525,-0.0403819,0.0364702 \backslash \mathrm{PG}=\mathrm{C} 01 \quad[\mathrm{X}(\mathrm{C} 3 \mathrm{H} 9 \mathrm{~N} 101)] \backslash \backslash \mathrm{e}$

\section{TEMPO-C $\left(\mathrm{CH}_{3}\right)_{3}$}

$1 \backslash 1 \backslash G I N C-A C 57 \backslash F O p t \backslash U B 3 L Y P \backslash 6-31 G(d) \backslash C 13 H 27 N 1 O 1 \backslash J O H 501 \backslash 21-F e b-2007 \backslash 0 \backslash \backslash \#$

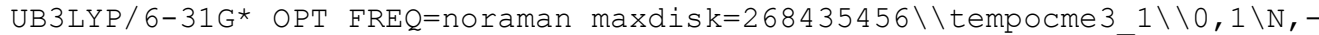
$0.1152876631,0.0504953763,-0.0515137461 \backslash 0,1.3172108254,0.12 \overline{3} 8374127,0$. $1532555182 \backslash \mathrm{C}, 2.0003099074,1.426121202,0.0724909067 \backslash \mathrm{C},-2.5201362032,-1$. $6209690022,-0.1851453552 \backslash \mathrm{H},-3.6026130274,-1.7207797256,-0.3356743058 \backslash \mathrm{H}$ $,-2.1435023347,-2.6319932947,0.0136491739 \backslash \mathrm{C}, 3.1586166629,1.2087766863$, $-0.9164247669 \backslash \mathrm{C}, 2.6075422596,1.7124526898,1.4585049794 \backslash \mathrm{C}, 1.1104992694$, $2.5878178386,-0.3687791558 \backslash C,-0.0532176399,-1.6901762601,1.8484565677 \backslash$ $C, 0.435915162,-2.1163958035,-1.3304769157 \backslash C,-0.729146799,-0.4399394389$ $, 1.2376397453 \backslash \mathrm{C},-0.3474843635,-0.7832766002,-1.2895897239 \backslash \mathrm{C},-0.6412715$ 
$032,0.6898342486,2.2794792965 \backslash C, 0.0678907144,0.042739517,-2.5204340496$ $\backslash C,-2.2356807661,-0.6933703234,0.9911506707 \backslash C,-1.8688330758,-1.0319900$ $152,-1.4314851791 \backslash \mathrm{H},-2.7233483432,0.2731403719,0.8030808684 \backslash \mathrm{H},-2.35567$ $78291,-0.074039431,-1.6604211244 \backslash \mathrm{H},-2.6656432395,-1.0890258561,1.91994$ $20606 \backslash \mathrm{H},-2.0227566907,-1.6806410776,-2.303097413 \backslash \mathrm{H},-1.2780906549,0.444$ $604057,3.1375132196 \backslash \mathrm{H},-0.2390287193,-0.4835154425,-3.4318191994 \backslash \mathrm{H},-0.9$ $885073181,1.6387275873,1.8589716652 \backslash \mathrm{H},-0.4192910539,1.0221180656,-2.51$ $45631499 \backslash \mathrm{H}, 0.3751271302,0.8165029583,2.6531485582 \backslash \mathrm{H}, 1.1484674107,0.183$ $7336462,-2.5719254139 \backslash \mathrm{H},-0.3717998737,-1.804709293,2.8913746597 \backslash \mathrm{H}, 0.42$ $02841266,-2.5192740559,-2.3500297966 \backslash \mathrm{H},-0.3074628858,-2.6164813695,1.3$ $293174338 \backslash \mathrm{H}, 0.0176753948,-2.8819200794,-0.6737796435 \backslash \mathrm{H}, 1.0328546615,-1$ $.5748973759,1.8348826472 \backslash \mathrm{H}, 1.4771035933,-1.9506509719,-1.0452894974 \backslash \mathrm{H}$, $1.7036516413,3.5094746355,-0.3410254168 \backslash \mathrm{H}, 0.7384406829,2.4562819025,-1$ $.3860804346 \backslash \mathrm{H}, 0.2521029483,2.7127698709,0.2939896936 \backslash \mathrm{H}, 3.3599674988,2$. $5062939037,1.3867013265 \backslash \mathrm{H}, 1.8535408239,2.0375893111,2.178739754 \backslash \mathrm{H}, 3.09$ $71980653,0.8136266961,1.8477363406 \backslash \mathrm{H}, 3.8199080476,2.0825174858,-0.9316$ $143421 \backslash \mathrm{H}, 3.7507924353,0.3374149865,-0.6166994206 \backslash \mathrm{H}, 2.7977407226,1.0427$ $349671,-1.9342166078 \backslash \backslash$ Version=IA64L-G03RevD.01 \State=1-A \HF=-641.57034 $41 \backslash S 2=0 . \backslash S 2-1=0 . \backslash S 2 A=0 . \backslash R M S D=5.153 e-09 \backslash R M S F=3.684 e-06 \backslash$ Therma $l=0 . \backslash$ Dipol $e=-0.1483104,0.0981592,-0.0378225 \backslash P G=C 01 \quad[X(C 13 H 27 N 1 O 1)] \backslash \backslash @$

\section{$\left(\mathrm{CH}_{3}\right)_{2} \mathrm{NO}-\mathrm{C}\left(\mathrm{CH}_{3}\right)_{3}$}

$1 \backslash 1 \backslash G I N C-A C 9 \backslash F O p t \backslash U B 3 L Y P \backslash 6-31 G(d) \backslash C 6 H 15 N 101 \backslash J O H 501 \backslash 28-M a r-2007 \backslash 0 \backslash \backslash \#$ UB

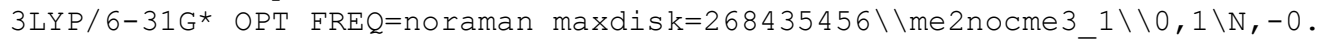
$0625107302,0.2678581594,-0.2209562111 \backslash 0,0.9973724851,-0.3785370443,0.5$ $248915255 \backslash C, 2.3044594446,-0.1571143764,-0.0654657243 \backslash C, 3.2309194729,-0$ $.9231549314,0.8844270873 \backslash C, 2.6470508736,1.3382817771,-0.0781397578 \backslash C, 2$ $.3655639203,-0.7430986908,-1.4820402288 \backslash \mathrm{C},-0.7065539053,1.1812348406,0$ $.7210477164 \backslash \mathrm{C},-0.9749855105,-0.8030296072,-0.6172001229 \backslash \mathrm{H}, 3.3681266628$ $,-0.6189235991,-1.9073770963 \backslash \mathrm{H}, 2.1319200924,-1.8135601328,-1.466823401$ $3 \backslash \mathrm{H}, 1.6508149143,-0.2360631099,-2.1367922012 \backslash \mathrm{H}, 3.6546331285,1.49924222$ $3,-0.478546964 \backslash \mathrm{H}, 1.9380958075,1.8877305389,-0.704352632 \backslash \mathrm{H}, 2.6137824909$ $, 1.7500296534,0.9367810532 \backslash \mathrm{H}, 4.2719414889,-0.84096595,0.5537556801 \backslash \mathrm{H}, 3$ $.1559309829,-0.5211516646,1.9001241676 \backslash \mathrm{H}, 2.9581160305,-1.983144282,0.9$ $139541418 \backslash \mathrm{H},-1.8241796389,-0.3558734217,-1.1456417976 \backslash \mathrm{H},-0.4598520209$, $-1.4841481194,-1.2978574998 \backslash \mathrm{H},-1.350765405,-1.3777183652,0.2453177124 \backslash$ $\mathrm{H},-1.5508870128,1.661670274,0.2144804014 \backslash \mathrm{H},-1.0749123498,0.6658563876$, $1.6233225144 \backslash \mathrm{H}, 0.005378778,1.9530394408,1.0211516371 \backslash \backslash$ Version=IA $64 \mathrm{~L}-\mathrm{G} 0$

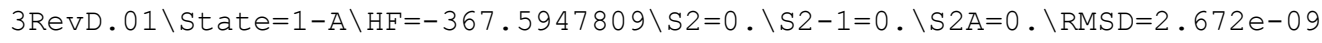
$\backslash \mathrm{RMSF}=2.863 \mathrm{e}-06 \backslash \mathrm{Thermal}=0 . \backslash \mathrm{Dipole}=-0.103784,0.1071399,-0.1379701 \backslash \mathrm{PG}=\mathrm{C} 0$ $1[\mathrm{X}(\mathrm{C} 6 \mathrm{H} 15 \mathrm{~N} 1 \mathrm{O} 1)] \backslash \backslash \mathrm{e}$

\section{$\mathrm{H}_{2} \mathrm{NO}-\mathrm{C}\left(\mathrm{CH}_{3}\right)_{3}$}

$1 \backslash 1 \backslash G I N C-A C 9 \backslash F O p t \backslash U B 3 L Y P \backslash 6-31 G(d) \backslash C 4 H 11 N 101 \backslash J 0 H 501 \backslash 28-M a r-2007 \backslash 0 \backslash \backslash \#$ UB $3 \mathrm{LYP} / 6-31 \mathrm{G} * \mathrm{OPT} F R E Q=$ noraman maxdisk $=268435456 \backslash \backslash \mathrm{h} 2 \mathrm{nocme} 3 \_1 \backslash \backslash 0,1 \backslash \mathrm{N}, 0.23$ $86410896,0.0575818083,0.5278607513 \backslash 0,1.3487213551,0.1321843947,-0.3915$ $034151 \backslash \mathrm{C}, 2.1647223008,1.3032155319,-0.1313414552 \backslash \mathrm{C}, 3.2599556993,1.1889$ $438295,-1.1960269599 \backslash \mathrm{C}, 2.7581357057,1.2316259797,1.2807878774 \backslash \mathrm{C}, 1.3367$ $599206,2.5790818879,-0.3260692557 \backslash \mathrm{H}, 0.3405012875,-0.8732822113,0.93737$ $38327 \backslash \mathrm{H},-0.5706806799,-0.0098049212,-0.0926530992 \backslash \mathrm{H}, 1.957242284,3.4710$ $107452,-0.1820955126 \backslash \mathrm{H}, 0.9188518169,2.612181094,-1.3387988131 \backslash \mathrm{H}, 0.5133$ $280781,2.6125930154,0.3930427817 \backslash \mathrm{H}, 3.4175793676,2.0865341201,1.4692740$ $277 \backslash \mathrm{H}, 1.9612558992,1.2395839184,2.0299358676 \backslash \mathrm{H}, 3.3441322123,0.31340231$ $16,1.4025781302 \backslash \mathrm{H}, 3.9612051475,2.0263437081,-1.1143053713 \backslash \mathrm{H}, 3.81888702$ $08,0.2551137444,-1.0743438598 \backslash \mathrm{H}, 2.8229914949,1.1989510433,-2.200135526$

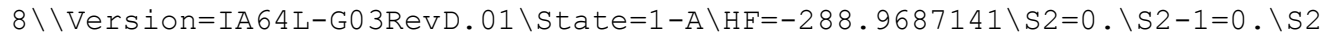
$\mathrm{A}=0 . \backslash \mathrm{RMSD}=6.809 \mathrm{e}-09 \backslash \mathrm{RMSF}=8.155 \mathrm{e}-06 \backslash \mathrm{Thermal}=0 . \backslash \mathrm{Dipole}=-0.1927705,-0.145$ $1375,0.0489236 \backslash P G=C 01 \quad[X(C 4 H 11 N 101)] \backslash \backslash @$

\section{TEMPO- $\mathrm{CH}_{2} \mathrm{CH}_{2} \mathrm{CH}_{3}$}

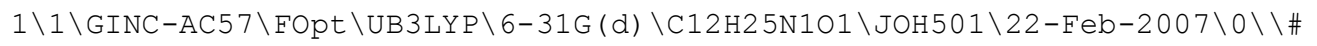


UB3LYP/6-31G* OPT FREQ=noraman maxdisk=402653184\\tempoch2ch2ch3 $1 \mathrm{~s} \backslash \backslash 0$ $, 1 \backslash \mathrm{N},-0.3237823115,0.1291034527,0 . \backslash 0,0.3211099002,1.4235631913,0 . \backslash \mathrm{C},-0$ $.6365594363,2.4852675753,0 . \backslash \mathrm{C},-0.2352947925,-2.7558091677,0 . \backslash \mathrm{H},-0.7576$ $414401,-3.7208390353,0 . \backslash \mathrm{H}, 0.8370353478,-2.9896132685,0 . \backslash \mathrm{C}, 0.1460221773$ $, 3.7942019323,0 . \backslash \mathrm{H},-1.2815722586,2.4199402748,0.885414702 \backslash \mathrm{H},-1.2815722$ $586,2.4199402748,-0.885414702 \backslash C, 1.5268920051,-0.6160871323,1.619242158$ $\backslash \mathrm{C}, 1.5268920051,-0.6160871323,-1.619242158 \backslash \mathrm{C}, 0.0167236,-0.5464082453,1$ $.2914464467 \backslash C, 0.0167236,-0.5464082453,-1.2914464467 \backslash C,-0.6723039173,0$. $2459875969,2.4196983704 \backslash \mathrm{C},-0.6723039173,0.2459875969,-2.4196983704 \backslash C_{,}-$ $0.613297031,-1.9576705725,1.2486746051 \backslash \mathrm{C},-0.613297031,-1.9576705725,-1$ $.2486746051 \backslash \mathrm{H},-1.7060430439,-1.8493253459,1.2794747761 \backslash \mathrm{H},-1.7060430439$ $,-1.8493253459,-1.2794747761 \backslash \mathrm{H},-0.3207600593,-2.4927245342,2.161055071$ $8 \backslash \mathrm{H},-0.3207600593,-2.4927245342,-2.1610550718 \backslash \mathrm{H},-0.5952287632,-0.30719$ $64312,3.3630336296 \backslash \mathrm{H},-0.5952287632,-0.3071964312,-3.3630336296 \backslash \mathrm{H},-1.73$ $27701185,0.3966404451,2.1932045499 \backslash \mathrm{H},-1.7327701185,0.3966404451,-2.193$ $2045499 \backslash \mathrm{H},-0.201669742,1.2225303808,2.5671610493 \backslash \mathrm{H},-0.201669742,1.2225$ $303808,-2.5671610493 \backslash \mathrm{H}, 1.6621052485,-0.8766012573,2.6756480471 \backslash \mathrm{H}, 1.662$ $1052485,-0.8766012573,-2.6756480471 \backslash \mathrm{H}, 2.0609864537,-1.3644691033,1.029$ $8030141 \backslash \mathrm{H}, 2.0609864537,-1.3644691033,-1.0298030141 \backslash \mathrm{H}, 1.993490683,0.357$ $0134862,1.4466255363 \backslash \mathrm{H}, 1.993490683,0.3570134862,-1.4466255363 \backslash \mathrm{C},-0.774$ $3785791,5.0193479296,0 . \backslash \mathrm{H}, 0.8022948785,3.8142816192,0.8793670451 \backslash \mathrm{H}, 0.8$ $022948785,3.8142816192,-0.8793670451 \backslash \mathrm{H},-0.193019596,5.9475854325,0 . \backslash \mathrm{H}$, $-1.4221335706,5.0330247922,-0.884992893 \backslash \mathrm{H},-1.4221335706,5.0330247922,0$

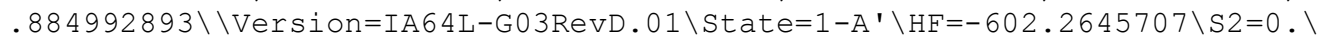
$\mathrm{S} 2-1=0 . \backslash \mathrm{S} 2 \mathrm{~A}=0 . \backslash \mathrm{RMSD}=7.067 \mathrm{e}-09 \backslash \mathrm{RMSF}=3.077 \mathrm{e}-06 \backslash \mathrm{Thermal}=0 . \backslash \mathrm{Dipole}=-0.1419$ $137,-0.0790552,0 . \backslash P G=C S \quad[S G(C 4 H 3 N 101), X(C 8 H 22)] \backslash \backslash @$

\section{$\left(\mathrm{CH}_{3}\right)_{2} \mathrm{NO}-\mathrm{CH}_{2} \mathrm{CH}_{2} \mathrm{CH}_{3}$}

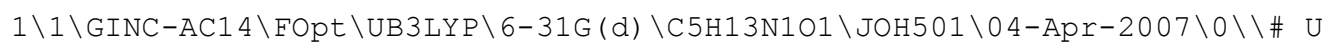
B3LYP/6-31G* OPT FREQ=noraman maxdisk=402653184 \me2noch2ch3 $2 \backslash \backslash 0,1 \backslash N$, $-0.18427977,-1.0203481275,0 . \backslash 0,0.3333581602,0.3332795781,0 . \backslash \bar{C},-0.75686$ $59692,1.2502531001,0 . \backslash \mathrm{C},-0.1726136295,2.6582638786,0 . \backslash \mathrm{H},-1.3883615745$, $1.0891623492,0.8869158697 \backslash \mathrm{H},-1.3883615745,1.0891623492,-0.8869158697 \backslash \mathrm{C}$ $, 0.345475721,-1.6430021931,1.2090124581 \backslash C, 0.345475721,-1.6430021931,-1$ $.2090124581 \backslash \mathrm{C},-1.2590845292,3.7383691068,0 . \backslash \mathrm{H}, 0.4736674315,2.771175052$ $2,0.8795554925 \backslash \mathrm{H}, 0.4736674315,2.7711750522,-0.8795554925 \backslash \mathrm{H},-0.00762834$ $41,-2.6792750508,1.2483598837 \backslash \mathrm{H}, 1.4476028323,-1.636362915,1.2436764062$ $\backslash \mathrm{H},-0.0395847858,-1.1106789258,2.0828336179 \backslash \mathrm{H},-0.0076283441,-2.6792750$ $508,-1.2483598837 \backslash \mathrm{H},-0.0395847858,-1.1106789258,-2.0828336179 \backslash \mathrm{H}, 1.4476$ $028323,-1.636362915,-1.2436764062 \backslash \mathrm{H},-0.8168766259,4.7404218281,0 . \backslash \mathrm{H},-1$ $.9020300986,3.6590770011,-0.8851261592 \backslash \mathrm{H},-1.9020300986,3.6590770011,0$. $8851261592 \backslash \backslash$ Version=IA6 4L-G03RevD.01 \State=1-A $\backslash \backslash H F=-328.2747966 \backslash S 2=0 . \backslash$ $\mathrm{S} 2-1=0 . \backslash \mathrm{S} 2 \mathrm{~A}=0 . \backslash \mathrm{RMSD}=1.868 \mathrm{e}-09 \backslash \mathrm{RMSF}=2.030 \mathrm{e}-05 \backslash \mathrm{Thermal}=0 . \backslash \mathrm{Dipole}=-0.0875$ $716,-0.1235704,0 . \backslash P G=C S \quad[S G(C 3 H 1 N 1 O 1), X(C 2 H 12)] \backslash \backslash @$

\section{TEMPO- $\mathrm{CH}_{2}\left(\mathrm{C}_{6} \mathrm{H}_{5}\right)$}

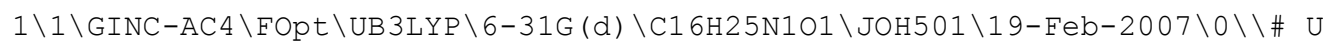

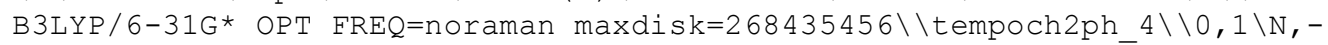
$0.2610848723,0.0166244196,0.2310293306 \backslash 0,1.0961278276,0.1169939508,0.7$ $240665326 \backslash \mathrm{C}, 1.5422697182,1.4812915609,0.7288869009 \backslash \mathrm{C},-2.6110654669,-1$. $5157595134,-0.4463182468 \backslash \mathrm{H},-3.6432944691,-1.5561195211,-0.8163807029 \backslash \mathrm{H}$ $,-2.3252915595,-2.5518573069,-0.2257179343 \backslash C, 2.8926244877,1.517620441$, $1.4041165223 \backslash \mathrm{H}, 0.8266090112,2.1149123141,1.2622159403 \backslash \mathrm{H}, 1.6100980898,1$ $.8529006678,-0.3019221937 \backslash \mathrm{C},-0.6017962921,-1.7752147112,2.0420569615 \backslash \mathrm{C}$ $, 0.4961317465,-2.1162318346,-0.9879966968 \backslash C,-1.1066258395,-0.480355583$ $, 1.36323481 \backslash \mathrm{C},-0.2305406983,-0.7522341895,-1.0527366006 \backslash \mathrm{C},-1.137876942$ $1,0.6282867498,2.4335344492 \backslash \mathrm{C}, 0.497992899,0.1195328163,-2.0945579101 \backslash \mathrm{C}$ $,-2.5420780158,-0.6561697465,0.8168626861 \backslash C,-1.6961456168,-0.919760185$ $9,-1.517145474 \backslash \mathrm{H},-2.9489874626,0.3381339041,0.5877719371 \backslash \mathrm{H},-2.08308044$ $9,0.0686010665,-1.8000808999 \backslash \mathrm{H},-3.1646914579,-1.0790744109,1.615356437$ $4 \backslash \mathrm{H},-1.7003291032,-1.5352093193,-2.4257320818 \backslash \mathrm{H},-1.8721443234,0.376459$ $2585,3.2074313863 \backslash \mathrm{H}, 0.3853992672,-0.3214500764,-3.0918469997 \backslash \mathrm{H},-1.4230$ $578285,1.587490837,1.9887670655 \backslash \mathrm{H}, 0.0773153756,1.1300386364,-2.1143425$ 
$608 \backslash \mathrm{H},-0.1645515323,0.7386816751,2.9204504295 \backslash \mathrm{H}, 1.5690166465,0.1883892$ $233,-1.882116059 \backslash \mathrm{H},-1.121360229,-1.9139153874,2.9975041049 \backslash \mathrm{H}, 0.6946785$ $19,-2.4746735672,-2.0049464576 \backslash \mathrm{H},-0.7788430023,-2.6716506492,1.4438240$ $021 \backslash \mathrm{H},-0.0831853579,-2.889249625,-0.4789260514 \backslash \mathrm{H}, 0.4692646562,-1.70064$ $56234,2.246210297 \backslash \mathrm{H}, 1.4540335202,-2.0099612698,-0.4723454145 \backslash \mathrm{C}, 3.94793$ $30917,0.7274578326,0.9268884807 \backslash C, 5.1980924619,0.76630005,1.5417815514$ $\backslash \mathrm{C}, 5.4144586645,1.6028083455,2.6403234969 \backslash \mathrm{C}, 3.1175135816,2.3454227236$, $2.5089755535 \backslash \mathrm{C}, 4.3716897148,2.3939601097,3.1219078007 \backslash \mathrm{H}, 3.7810026097,0$ $.0735494566,0.0753549333 \backslash \mathrm{H}, 6.0063610092,0.1458949281,1.1631435653 \backslash \mathrm{H}, 6$. $3903909305,1.6355232987,3.1175732251 \backslash \mathrm{H}, 2.3048958819,2.9584139958,2.892$ $4433461 \backslash \mathrm{H}, 4.530310808,3.0449942589,3.9777105378 \backslash \backslash$ Version=IA64L-G03RevD $.01 \backslash$ State $=1-A \backslash H F=-754.6843944 \backslash S 2=0 . \backslash S 2-1=0 . \backslash \mathrm{S} 2 A=0 . \backslash \mathrm{RMSD}=4.183 e-09 \backslash \mathrm{RMSF}$ $=3.660 e-06 \backslash$ Thermal $=0 . \backslash$ Dipole $=-0.1835205,0.0230823,-0.0857975 \backslash P G=C 01 \quad[X$ (C16H25N101) ] \\@

\section{$\left(\mathrm{CH}_{3}\right)_{2} \mathrm{NO}-\mathrm{CH}_{2}\left(\mathrm{C}_{6} \mathrm{H}_{5}\right)$}

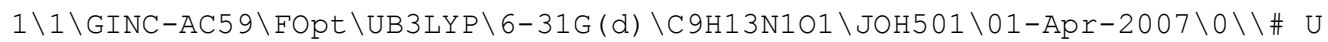
B3LYP/6-31G* OPT FREQ=noraman maxdisk=268435456\\me2noch2ph_1 $\backslash \backslash 0,1 \backslash N$, $0.0155181456,0.0766997915,-0.0119833666 \backslash 0,1.4239641777,-0.0022614766,0$ $.1485371689 \backslash \mathrm{C}, 1.9486119598,1.322694701,0.2386697456 \backslash \mathrm{C}, 3.4563725374,1.2$ $386620674,0.2844432234 \backslash \mathrm{H}, 1.5621739867,1.8295246746,1.1342663002 \backslash \mathrm{H}, 1.61$ $50651841,1.9046858485,-0.633613331 \backslash \mathrm{C},-0.5754391216,-0.6461227166,1.126$ $1420413 \backslash \mathrm{C},-0.3072184808,-0.5891459631,-1.2775758289 \backslash \mathrm{C}, 4.1516037708,0.4$ $1298652,-0.6094074063 \backslash \mathrm{C}, 5.5446365425,0.3636647141,-0.5901594543 \backslash \mathrm{C}, 6.26$ $37720895,1.1457519201,0.3174314929 \backslash C, 4.1839401192,2.010108543,1.196452$ $0121 \backslash \mathrm{C}, 5.5797845914,1.9707742217,1.2104331315 \backslash \mathrm{H}, 3.5918047335,-0.201405$ $7218,-1.3081836847 \backslash \mathrm{H}, 6.0712314869,-0.2853311763,-1.2852898866 \backslash \mathrm{H}, 7.3499$ $221261,1.1083185142,0.3301032557 \backslash \mathrm{H}, 3.6545532653,2.6461717303,1.9025805$ $954 \backslash \mathrm{H}, 6.1302225523,2.577745402,1.9246019667 \backslash \mathrm{H},-1.6676761963,-0.6235166$ $429,1.0458758938 \backslash \mathrm{H},-0.2387906564,-1.6948495435,1.1724463977 \backslash \mathrm{H},-0.28325$ $41678,-0.1394129864,2.0495699929 \backslash \mathrm{H},-1.3910304404,-0.571785875,-1.43562$ $68157 \backslash \mathrm{H}, 0.1709552303,-0.0366865727,-2.0909631602 \backslash \mathrm{H}, 0.0432928553,-1.634$ $2699736,-1.2997202835 \backslash \backslash$ Version=IA64 L-G03RevD.01 \State=1-A \HF=-480.6949 $46 \backslash \mathrm{S} 2=0 . \backslash \mathrm{S} 2-1=0 . \backslash \mathrm{S} 2 \mathrm{~A}=0 . \backslash \mathrm{RMSD}=3.964 \mathrm{e}-09 \backslash \mathrm{RMSF}=2.239 \mathrm{e}-06 \backslash$ Therma $\mathrm{l}=0 . \backslash \mathrm{Dipol}$ $e=-0.2609131,0.0003553,-0.0593945 \backslash P G=C 01 \quad[X(C 9 H 13 N 101)] \backslash \backslash @$

\section{$\mathrm{H}_{2} \mathrm{NO}-\mathrm{CH}_{2}\left(\mathrm{C}_{6} \mathrm{H}_{5}\right)$}

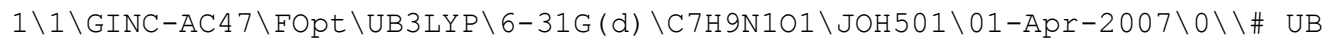

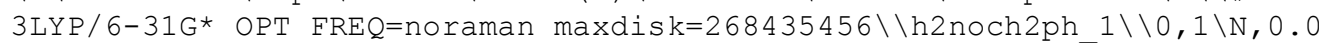
$065814907,0.0819194299,-0.2012730007 \backslash 0,1.4261985715,-0.0018366896,0.05$ $43151173 \backslash \mathrm{C}, 1.9460605692,1.3265819357,0.1344117914 \backslash \mathrm{C}, 3.4494847839,1.238$ $7419479,0.2446089975 \backslash \mathrm{H}, 1.5234235953,1.8535728464,1.0019409827 \backslash \mathrm{H}, 1.6463$ $725887,1.8850035234,-0.7644293023 \backslash \mathrm{H},-0.4032200618,-0.4103131936,0.5965$ $702783 \backslash \mathrm{H},-0.1186867071,-0.5499571756,-0.995402227 \backslash \mathrm{C}, 4.1818648563,0.450$ $2792676,-0.6533999464 \backslash C, 5.5722280445,0.3923035276,-0.5741954798 \backslash C, 6.25$ $19963208,1.1286734024,0.3998639696 \backslash C, 4.1375135709,1.9637913209,1.22268$ $22965 \backslash \mathrm{C}, 5.5313399226,1.9161382569,1.2977404099 \backslash \mathrm{H}, 3.6525774453,-0.12685$ $44906,-1.4060726357 \backslash \mathrm{H}, 6.1277906381,-0.2263568565,-1.2743976266 \backslash \mathrm{H}, 7.336$ $3640764,1.085240328,0.4593614088 \backslash \mathrm{H}, 3.5783678884,2.5703475427,1.9317524$ $644 \backslash \mathrm{H}, 6.0511624063,2.4875350766,2.0624025018 \backslash \backslash$ Version=IA64L-G0 3RevD. 01 $\backslash$ State $=1-\mathrm{A} \backslash \mathrm{HF}=-402.0673549 \backslash \mathrm{S} 2=0 . \backslash \mathrm{S} 2-1=0 . \backslash \mathrm{S} 2 \mathrm{~A}=0 . \backslash \mathrm{RMSD}=3.044 \mathrm{e}-09 \backslash \mathrm{RMSF}=1$. $956 e-06 \backslash$ Thermal $=0 . \backslash$ Dipole $=-0.1981384,-0.1959662,-0.0243245 \backslash P G=C 01 \quad[X(C$ $7 \mathrm{H} 9 \mathrm{~N} 101)] \backslash \backslash$ e

\section{TEMPO- $\mathrm{CH}_{2} \mathrm{OH}$}

$1 \backslash 1 \backslash G I N C-A C 28 \backslash F O p t \backslash U B 3 L Y P \backslash 6-31 G(d) \backslash C 10 H 21 N 102 \backslash J O H 501 \backslash 21-F e b-2007 \backslash 0 \backslash \backslash \#$

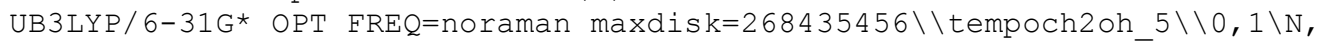
$0.0315319237,0.0258900943,0.0192178627 \backslash 0,1.4846158414,-0.0074713401,0$. $0315101516 \backslash \mathrm{C}, 2.0568453042,1.2873734748,-0.0628518368 \backslash \mathrm{C},-2.5062927872,-$ $1.3810400645,-0.0520794577 \backslash \mathrm{H},-3.6032740323,-1.3614856643,-0.0670595428$ $\backslash \mathrm{H},-2.2192317273,-2.4396677007,-0.0739107553 \backslash 0,2.191221766,1.972950121$ 
$2,1.1523398533 \backslash \mathrm{H}, 1.4934688073,1.8826051679,-0.7884347885 \backslash \mathrm{H}, 3.074032511$ $3,1.1037176854,-0.4184005461 \backslash \mathrm{C}, 0.1789421715,-1.9946088485,1.6049006614$ $\backslash \mathrm{C}, 0.2157269359,-1.936831386,-1.6350623862 \backslash \mathrm{C},-0.4470264818,-0.61526971$ $96,1.2928678491 \backslash C,-0.4092969933,-0.5648015822,-1.292752637 \backslash C,-0.097412$ $9265,0.3296723918,2.4570650797 \backslash C,-0.0098012773,0.4206293457,-2.4089735$ $291 \backslash \mathrm{C},-1.9894749039,-0.7050384813,1.2168905572 \backslash \mathrm{C},-1.9529028595,-0.6447$ $91241,-1.2712415346 \backslash \mathrm{H},-2.3978148612,0.3139758152,1.2608849634 \backslash \mathrm{H},-2.353$ $8801065,0.3780708163,-1.2774270693 \backslash \mathrm{H},-2.3436697082,-1.2269310351,2.114$ $5507505 \backslash \mathrm{H},-2.2824572536,-1.120578996,-2.2033668328 \backslash \mathrm{H},-0.5454688474,-0$. $0451442558,3.38422305 \backslash \mathrm{H},-0.467510187,0.1086164028,-3.3547356531 \backslash \mathrm{H},-0.5$ $065427865,1.3312021937,2.272353412 \backslash \mathrm{H},-0.3575776717,1.4330473165,-2.178$ $6430323 \backslash \mathrm{H}, 0.9818944482,0.4061502782,2.6026810547 \backslash \mathrm{H}, 1.0734865042,0.4438$ $38104,-2.5602475414 \backslash \mathrm{H},-0.0255786585,-2.2590879728,2.648761837 \backslash \mathrm{H}, 0.0478$ $18816,-2.1599479503,-2.6952017005 \backslash \mathrm{H},-0.2195106752,-2.7992353907,0.9833$ $558861 \backslash \mathrm{H},-0.2109643172,-2.7598931666,-1.0588507511 \backslash \mathrm{H}, 1.2624609126,-1.9$ $577029591,1.4703379536 \backslash \mathrm{H}, 1.2940283144,-1.9162637041,-1.4594746106 \backslash \mathrm{H}, 1$. $3080848053,2.2782322511,1.4123032829 \backslash \backslash$ Version=IA64L-G03RevD. 01 \State=1 $-\mathrm{A} \backslash \mathrm{HF}=-598.8500349 \backslash \mathrm{S} 2=0 . \backslash \mathrm{S} 2-1=0 . \backslash \mathrm{S} 2 \mathrm{~A}=0 . \backslash \mathrm{RMSD}=9.741 \mathrm{e}-09 \backslash \mathrm{RMSF}=2.844 \mathrm{e}-06 \backslash$ Thermal $=0 . \backslash$ Dipole $=-0.7976459,-0.0728131,-0.3098766 \backslash P G=C 01 \quad[X(C 10 H 21 N 10$ 2) $] \backslash \backslash @$

\section{$\left(\mathrm{CH}_{3}\right)_{2} \mathrm{NO}-\mathrm{CH}_{2} \mathrm{OH}$}

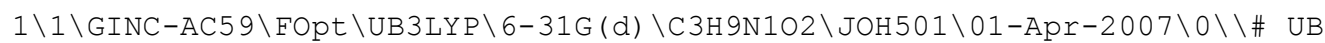

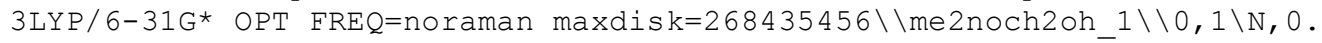
$0526527458,0.0846241804,0.0338656162 \backslash 0,1.4981596453,-0.0400508357,-0.0$ $543709193 \backslash \mathrm{C}, 2.0433710305,1.2657439282,0.0112576402 \backslash 0,1.7862834577,1.91$ $85146487,1.2206881468 \backslash \mathrm{H}, 1.6767826304,1.8563911667,-0.8432244981 \backslash \mathrm{H}, 3.12$ $38066001,1.1219008626,-0.063890414 \backslash \mathrm{C},-0.3382167587,-0.7537908786,1.166$ $3061516 \backslash \mathrm{C},-0.4725815616,-0.424521078,-1.2309077258 \backslash \mathrm{H}, 0.827593002,2.079$ $7791527,1.226975553 \backslash \mathrm{H},-1.5657826667,-0.3633783072,-1.2012952322 \backslash \mathrm{H},-0.1$ $107009083,0.2038800262,-2.0489299188 \backslash \mathrm{H},-0.1729767219,-1.4673612014,-1$. $4206292511 \backslash \mathrm{H},-1.4267759156,-0.7000598947,1.2764638916 \backslash \mathrm{H},-0.0405398662$, $-1.8059036927,1.032539677 \backslash \mathrm{H}, 0.1354152871,-0.3631080774,2.069131283 \backslash \backslash \mathrm{Ve}$

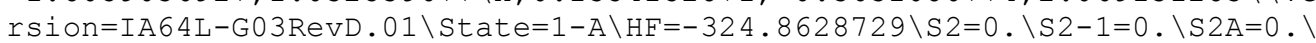
$\operatorname{RMSD}=8.270 \mathrm{e}-09 \backslash \mathrm{RMSF}=2.670 \mathrm{e}-05 \backslash \mathrm{Thermal}=0 . \backslash \mathrm{Dipole}=-0.7717084,-0.1342466$, $-0.4063258 \backslash P G=C 01 \quad[X(C 3 H 9 N 102)] \backslash \backslash Q$

\section{$\mathrm{H}_{2} \mathrm{NO}-\mathrm{CH}_{2} \mathrm{OH}$}

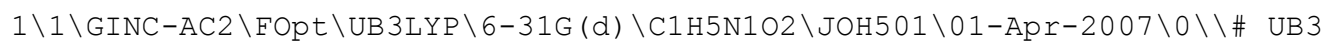

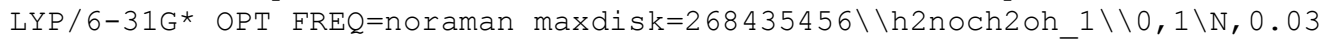
$84293765,0.1451349308,0.1233268414 \backslash 0,1.4497271576,0.0091512517,-0.1621$ $173999 \backslash \mathrm{C}, 2.0256169651,1.2998399218,-0.0415362864 \backslash 0,1.9554763868,1.8216$ $706271,1.2510147655 \backslash \mathrm{H}, 1.5519461644,1.9743995007,-0.7718248189 \backslash \mathrm{H}, 3.0810$ $204152,1.1537790317,-0.283265294 \backslash \mathrm{H},-0.1172819799,-0.5502295743,0.85505$ $77599 \backslash \mathrm{H},-0.4106160473,-0.2330360006,-0.7134779391 \backslash \mathrm{H}, 1.0113615615,1.985$ $5103111,1.4151123714 \backslash \backslash$ Version=IA64L-G03RevD.01 \State $=1-A \backslash H F=-246.23536$ $29 \backslash \mathrm{S} 2=0 . \backslash \mathrm{S} 2-1=0 . \backslash \mathrm{S} 2 \mathrm{~A}=0 . \backslash \mathrm{RMSD}=4.113 \mathrm{e}-09 \backslash \mathrm{RMSF}=4.733 \mathrm{e}-05 \backslash \mathrm{Therma} \mathrm{l}=0 . \backslash \mathrm{Dipol}$ $\mathrm{e}=-0.8006746,-0.2588729,-0.3304112 \backslash \mathrm{PG}=\mathrm{C} 01 \quad[\mathrm{X}(\mathrm{C} 1 \mathrm{H} 5 \mathrm{~N} 1 \mathrm{O} 2)] \backslash \backslash \mathrm{C}$

\section{TEMPO- $\mathrm{CH}_{2} \mathrm{CN}$}

$1 \backslash 1 \backslash G I N C-A C 7 \backslash F O p t \backslash U B 3 L Y P \backslash 6-31 G(d) \backslash C 11 H 20 N 201 \backslash J O H 501 \backslash 21-F e b-2007 \backslash 0 \backslash \backslash \# U$ B3LYP/6-31G* OPT FREQ=noraman maxdisk=268435456\\tempoch2cn_1s $\backslash \backslash 0,1 \backslash N$, $-0.0006082585,0.0001209881,0 . \backslash 0,1.4524385644,-0.0000661756, \overline{0} . \backslash \mathrm{C}, 1.9262$ $806309,1.3414927706,0 . \backslash \mathrm{C},-2.5358379333,-1.365021696,0 . \backslash \mathrm{H},-3.6321094172$ $,-1.324590487,0 . \backslash \mathrm{H},-2.2700569342,-2.4293116296,0 . \backslash \mathrm{C}, 3.3964774394,1.287$ $9030353,0 . \backslash \mathrm{H}, 1.5879616986,1.8903920127,0.8864827822 \backslash \mathrm{H}, 1.5879616986,1.8$ $903920127,-0.8864827822 \backslash \mathrm{C}, 0.1655488195,-1.9924712478,1.6172827684 \backslash \mathrm{C}, 0$. $1655488195,-1.9924712478,-1.6172827684 \backslash C,-0.445511538,-0.6094345156,1$. $2950140096 \backslash \mathrm{C},-0.445511538,-0.6094345156,-1.2950140096 \backslash \mathrm{C},-0.0388416368$, $0.3591193497,2.4229085765 \backslash C,-0.0388416368,0.3591193497,-2.4229085765 \backslash \mathrm{C}$ $,-1.9902797049,-0.6717713804,1.2496046663 \backslash \mathrm{C},-1.9902797049,-0.671771380$ 
$4,-1.2496046663 \backslash \mathrm{H},-2.3788590712,0.3550723062,1.2813337866 \backslash \mathrm{H},-2.3788590$ $712,0.3550723062,-1.2813337866 \backslash \mathrm{H},-2.3388189441,-1.17257491,2.161375162$ $5 \backslash \mathrm{H},-2.3388189441,-1.17257491,-2.1613751625 \backslash \mathrm{H},-0.4911995585,0.03774416$ $06,3.3677730894 \backslash \mathrm{H},-0.4911995585,0.0377441606,-3.3677730894 \backslash \mathrm{H},-0.385945$ $7804,1.3751778672,2.2069332287 \backslash \mathrm{H},-0.3859457804,1.3751778672,-2.2069332$ $287 \backslash \mathrm{H}, 1.0459590343,0.3747409537,2.5669895254 \backslash \mathrm{H}, 1.0459590343,0.37474095$ $37,-2.5669895254 \backslash \mathrm{H},-0.0121068817,-2.2308072641,2.6722959909 \backslash \mathrm{H},-0.01210$ $68817,-2.2308072641,-2.6722959909 \backslash \mathrm{H},-0.2647294175,-2.802571803,1.02543$ $50428 \backslash \mathrm{H},-0.2647294175,-2.802571803,-1.0254350428 \backslash \mathrm{H}, 1.2458944683,-1.980$ $245423,1.4518664422 \backslash \mathrm{H}, 1.2458944683,-1.980245423,-1.4518664422 \backslash \mathrm{N}, 4.5562$ $229331,1.2721329821,0 . \backslash \backslash$ Version=IA64L-G03RevD.01 $\backslash$ State $=1-A^{\prime} \backslash \mathrm{HF}=-615.86$ $66239 \backslash \mathrm{S} 2=0 . \backslash \mathrm{S} 2-1=0 . \backslash \mathrm{S} 2 \mathrm{~A}=0 . \backslash \mathrm{RMSD}=2.717 \mathrm{e}-09 \backslash \mathrm{RMSF}=2.934 \mathrm{e}-05 \backslash$ Thermal=0. $\backslash \mathrm{Di}$ pole $=-1.8211693,-0.0825748,0 . \backslash P G=C S \quad[S G(C 3 H 2 N 2 O 1), X(C 8 H 18)] \backslash \backslash @$

\section{$\left(\mathrm{CH}_{3}\right)_{2} \mathrm{NO}-\mathrm{CH}_{2} \mathrm{CN}$}

$1 \backslash 1 \backslash G I N C-A C 10 \backslash F O p t \backslash U B 3 L Y P \backslash 6-31 G(d) \backslash C 4 H 8 N 201 \backslash J O H 501 \backslash 27-M a r-2007 \backslash 0 \backslash \backslash \# \quad U B$ $3 \mathrm{LYP} / 6-31 \mathrm{G}^{*} \mathrm{OPT} \mathrm{FREQ}=$ noraman maxdisk$=268435456 \backslash \backslash$ me 2 noch $2 \mathrm{cn} 1 \backslash \backslash 0,1 \backslash \mathrm{N},-0$ $.0126966172,0.0829776742,0 . \backslash 0,1.4413768028,0.012764309,0 . \backslash \bar{C}, 1.92702194$ $86,1.3462408401,0 . \backslash \mathrm{C}, 3.3966135699,1.2940387947,0 . \backslash \mathrm{H}, 1.5859655076,1.897$ $5792126,0.8872536404 \backslash \mathrm{H}, 1.5859655076,1.8975792126,-0.8872536404 \backslash \mathrm{C},-0.43$ $47337113,-0.6159573876,1.2120842359 \backslash C,-0.4347337113,-0.6159573876,-1.2$ $120842359 \backslash \mathrm{N}, 4.5562572443,1.2820122368,0 . \backslash \mathrm{H},-1.5289128787,-0.6011051134$ $, 1.2491072862 \backslash \mathrm{H},-0.0849007271,-1.6596467597,1.2435358354 \backslash \mathrm{H},-0.04972466$ $39,-0.0839918793,2.0858265187 \backslash \mathrm{H},-1.5289128787,-0.6011051134,-1.2491072$ $862 \backslash \mathrm{H},-0.0497246639,-0.0839918793,-2.0858265187 \backslash \mathrm{H},-0.0849007271,-1.659$ $6467597,-1.2435358354 \backslash \backslash$ Version=IA64 L-G03RevD.01 \State=1-A $\backslash$ HF $=-341.876$ $0686 \backslash \mathrm{S} 2=0 . \backslash \mathrm{S} 2-1=0 . \backslash \mathrm{S} 2 \mathrm{~A}=0 . \backslash \mathrm{RMSD}=6.952 \mathrm{e}-09 \backslash \mathrm{RMSF}=1.154 \mathrm{e}-05 \backslash \mathrm{Therma} I=0 . \backslash \mathrm{Dip}$ ole $=-1.7722645,-0.040556,0 . \backslash P G=C S \quad[S G(C 2 N 201), x(C 2 H 8)] \backslash \backslash @$

\section{$\mathrm{H}_{2} \mathrm{NO}-\mathrm{CH}_{2} \mathrm{CN}$}

$1 \backslash 1 \backslash G I N C-A C 26 \backslash F O p t \backslash U B 3 L Y P \backslash 6-31 G(d) \backslash C 2 H 4 N 201 \backslash J O H 501 \backslash 01-A p r-2007 \backslash 0 \backslash \backslash \#$ UB

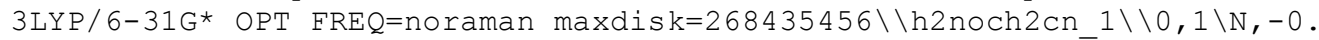
$0165978794,0.0880291465,0 . \backslash 0,1.4259101829,0.005053581,0 . \backslash \bar{C}, 1.917795906$ $5,1.3381283222,0 . \backslash \mathrm{C}, 3.3862508003,1.2742400848,0 . \backslash \mathrm{H}, 1.5773506924,1.8891$ $569997,0.8874876405 \backslash \mathrm{H}, 1.5773506924,1.8891569997,-0.8874876405 \backslash \mathrm{H},-0.277$ $974998,-0.473547182,0.8134814071 \backslash \mathrm{H},-0.277974998,-0.473547182,-0.813481$ $4071 \backslash \mathrm{N}, 4.5456895976,1.2528992315,0 . \backslash \backslash$ Version=IA64L-G03RevD.01 \State=1$A^{\prime} \backslash H F=-263.2483743 \backslash S 2=0 . \backslash S 2-1=0 . \backslash S 2 A=0 . \backslash R M S D=2.320 e-09 \backslash R M S F=6.311 e-05 \backslash$ Thermal $=0 . \backslash$ Dipole $=-1.6613553,-0.1687876,0 . \backslash \mathrm{PG}=\mathrm{CS} \quad[\mathrm{SG}(\mathrm{C} 2 \mathrm{~N} 2 \mathrm{O} 1), \mathrm{X}(\mathrm{H} 4)] \backslash \backslash @$

\section{TEMPO-CH}

$1 \backslash 1 \backslash G I N C-A C 10 \backslash F O p t \backslash U B 3 L Y P \backslash 6-31 G(d) \backslash C 10 H 20 F 1 N 101 \backslash J O H 501 \backslash 19-F e b-2007 \backslash 0 \backslash \backslash$ \# UB3LYP/6-31G* OPT FREQ=noraman maxdisk=268435456\\tempoch2f_2 $\backslash \backslash 0,1 \backslash \mathrm{N}$ $,-0.0046247068,0.0090319011,0.0156028624 \backslash 0,1.4530033004,0.002 \overline{2} 522183,-$ $0.018769646 \backslash \mathrm{C}, 1.9877564704,1.279156331,-0.055108122 \backslash \mathrm{C},-2.5350336291,-1$ $.3774068789,0.0131293887 \backslash \mathrm{H},-3.6318667862,-1.3502779433,0.0212937497 \backslash \mathrm{H}$, $-2.2559900333,-2.4383512705,-0.0033726405 \backslash F, 2.2455272921,1.7827385402$, $1.2130844029 \backslash \mathrm{H}, 1.3133896211,1.9776526627,-0.5545253397 \backslash \mathrm{H}, 2.9523105498$, $1.1881327306,-0.5661500464 \backslash \mathrm{C}, 0.1802847476,-1.9975336837,1.6132142383 \backslash \mathrm{C}$ $, 0.1518207157,-1.9612253962,-1.6316661708 \backslash C,-0.4418986247,-0.615397403$ $3,1.3082987729 \backslash C,-0.4621020376,-0.5855909955,-1.2836051059 \backslash C,-0.051494$ $1513,0.3432995934,2.4480606935 \backslash C,-0.0764216723,0.4013950825,-2.4031708$ $347 \backslash C,-1.9865127421,-0.6928410549,1.2652019327 \backslash C,-2.0056333915,-0.6598$ $589299,-1.2286778074 \backslash \mathrm{H},-2.3841103027,0.3299845224,1.3107954056 \backslash \mathrm{H},-2.40$ $24034931,0.364244999,-1.2397634299 \backslash \mathrm{H},-2.3248119392,-1.2054244529,2.174$ $1665914 \backslash \mathrm{H},-2.3562716695,-1.1472928363,-2.1469599708 \backslash \mathrm{H},-0.5345105143,0$. $0180158442,3.3767339991 \backslash \mathrm{H},-0.5360691225,0.087475876,-3.3471784636 \backslash \mathrm{H},-0$ $.3785569005,1.3631033165,2.2231652571 \backslash \mathrm{H},-0.4299879564,1.4111983311,-2$. $170009686 \backslash \mathrm{H}, 1.0270453859,0.3623421608,2.6083401788 \backslash \mathrm{H}, 1.0064031813,0.43$ $25717224,-2.5597042558 \backslash \mathrm{H}, 0.0065183143,-2.2476325315,2.6661483763 \backslash \mathrm{H},-0$. $0409298091,-2.1907677022,-2.6861476386 \backslash \mathrm{H},-0.2435058543,-2.8063097625,1$ 
$.0136472682 \backslash \mathrm{H},-0.2649097473,-2.7781374817,-1.0396135048 \backslash \mathrm{H}, 1.2602951051$ $,-1.9734953727,1.4473251594 \backslash \mathrm{H}, 1.2343804003,-1.9459321362,-1.481925614 \backslash$ $\backslash$ Version=IA64L-G03RevD.01 \State $=1-\mathrm{A} \backslash \mathrm{HF}=-622.8725255 \backslash \mathrm{S} 2=0 . \backslash \mathrm{S} 2-1=0 . \backslash \mathrm{S} 2 \mathrm{~A}=$ $0 . \backslash \mathrm{RMSD}=2.753 \mathrm{e}-09 \backslash \mathrm{RMSF}=7.789 \mathrm{e}-06 \backslash \mathrm{Thermal}=0 . \backslash \mathrm{Dipole}=-0.4745377,-0.26460$ $08,-0.5713065 \backslash \mathrm{PG}=\mathrm{C} 01 \quad[\mathrm{X}(\mathrm{C} 10 \mathrm{H} 20 \mathrm{~F} 1 \mathrm{~N} 101)] \backslash \backslash @$

\section{$\left(\mathrm{CH}_{3}\right)_{2} \mathrm{NO}-\mathrm{CH}_{2} \mathrm{~F}$}

$1 \backslash 1 \backslash G I N C-A C 10 \backslash F O p t \backslash U B 3 L Y P \backslash 6-31 G(d) \backslash C 3 H 8 F 1 N 101 \backslash J O H 501 \backslash 27-M a r-2007 \backslash 0 \backslash \backslash \#$ UB3LYP/6-31G* OPT FREQ=noraman maxdisk=268435456\\me2noch2f $1 \backslash \backslash 0,1 \backslash N,-$ $0.0091972911,0.0939162161,-0.1008358392 \backslash 0,1.4471812228,0.03 \overline{6} 6736852,-0$ $.1929995637 \backslash \mathrm{C}, 1.9789106238,1.2842211716,0.0641923915 \backslash \mathrm{F}, 1.9663973407,1$. $5777580278,1.4223416527 \backslash \mathrm{H}, 1.4152487258,2.0726471388,-0.4445605984 \backslash \mathrm{H}, 3$. $022503405,1.2359726841,-0.2631119005 \backslash C,-0.3638039232,-0.5798062965,1.1$ $492188779 \backslash \mathrm{C},-0.4831660907,-0.6391518628,-1.2703773578 \backslash \mathrm{H},-1.4546410127$, $-0.5608610266,1.2446509245 \backslash \mathrm{H},-0.0155858006,-1.6245525264,1.180886101 \backslash \mathrm{H}$ $, 0.0769135101,-0.0255881699,1.97877885 \backslash \mathrm{H},-1.5774720537,-0.6492701911,-$ $1.2411904277 \backslash \mathrm{H},-0.1609041012,-0.118595654,-2.1756432413 \backslash \mathrm{H},-0.114244555$ $,-1.6772331962,-1.3006098688 \backslash \backslash$ Version=IA64L-G03RevD.01 \State=1-A \HF=-3 $48.8833035 \backslash \mathrm{S} 2=0 . \backslash \mathrm{S} 2-1=0 . \backslash \mathrm{S} 2 \mathrm{~A}=0 . \backslash \mathrm{RMSD}=9.877 \mathrm{e}-09 \backslash \mathrm{RMSF}=5.716 \mathrm{e}-06 \backslash \mathrm{Thermal}=$ $0 . \backslash$ Dipole $=-0.3565955,-0.2211708,-0.4930925 \backslash P G=C 01 \quad[X(C 3 H 8 F 1 N 101)] \backslash \backslash @$

\section{$\mathrm{H}_{2} \mathrm{NO}-\mathrm{CH}_{2} \mathrm{~F}$}

$1 \backslash 1 \backslash G I N C-A C 59 \backslash F O p t \backslash U B 3 L Y P \backslash 6-31 G(d) \backslash C 1 H 4 F 1 N 101 \backslash J O H 501 \backslash 01-A p r-2007 \backslash 0 \backslash \backslash \#$

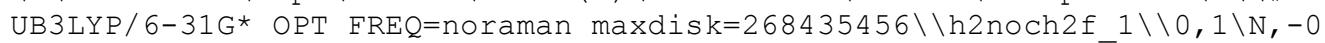
$.010377686,0.1020620243,-0.0522534948 \backslash 0,1.4253405846,0.0255401689,-0.2$ $480362203 \backslash \mathrm{C}, 1.9728708766,1.2741163219,-0.0263516025 \backslash \mathrm{F}, 2.0121948394,1.5$ $718240234,1.3266783835 \backslash \mathrm{H}, 1.3900582294,2.0584333279,-0.5198863806 \backslash \mathrm{H}, 3.0$ $005748191,1.2134126324,-0.3984238822 \backslash \mathrm{H},-0.1467692764,-0.2993662832,0.8$ $7901776 \backslash \mathrm{H},-0.3448723867,-0.6077122156,-0.706004563 \backslash \backslash$ Version=IA64L-G03R evD.01 State $=1-A \backslash H F=-270.2550308 \backslash S 2=0 . \backslash S 2-1=0 . \backslash S 2 A=0 . \backslash R M S D=4.805 e-09 \backslash R$ $\mathrm{MSF}=1.575 \mathrm{e}-05 \backslash \mathrm{Thermal}=0 . \backslash \mathrm{Dipole}=-0.2850761,-0.4081527,-0.3746364 \backslash \mathrm{PG}=\mathrm{C} 0$ $1[\mathrm{X}(\mathrm{C} 1 \mathrm{H} 4 \mathrm{~F} 1 \mathrm{~N} 1 \mathrm{O} 1)] \backslash \backslash @$

\section{TABLE S5: Geometries of the additional species in Table 7}

NOTE: All species had zero imaginary frequencies, as determined from frequency calculations at the B3-LYP/6-31G(d) level

\section{$\mathrm{CH}_{3} \mathrm{CH}_{2} \mathrm{CH}_{2} \mathrm{CH}_{2} \mathrm{CH}_{2} \mathrm{CH}_{2}{ }^{\bullet}$}

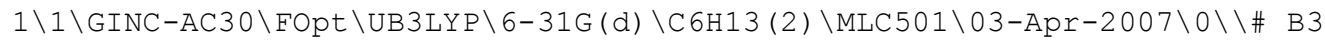
LYP/6-31G* Opt=tight INT (grid=ultrafine) $F R E Q=$ noraman maxdisk $=40265318$ $4 \backslash \backslash$ hexyl-geom $\backslash \backslash 0,2 \backslash C,-1.369140156,0.4816801814,0.0217328087 \backslash C,-2.60574$ $6806,-0.3515604111,-0.019698864 \backslash C,-0.0676420848,-0.336485442,-0.042566$ $9964 \backslash \mathrm{C}, 1.1982983415,0.5244916228,0.0412934337 \backslash \mathrm{H},-2.6246788294,-1.33605$ $3854,0.4398243736 \backslash \mathrm{H},-3.550547786,0.0612195893,-0.3594281378 \backslash \mathrm{H},-1.35056$ $12307,1.0901633406,0.947390599 \backslash \mathrm{H},-1.3850976285,1.2158050824,-0.7976966$ $433 \backslash \mathrm{H},-0.0559955799,-0.9197570978,-0.9737896703 \backslash \mathrm{H},-0.0654836877,-1.070$ $0634136,0.7771298807 \backslash \mathrm{C}, 2.4976980178,-0.2886895038,-0.0197244926 \backslash \mathrm{H}, 1.18$ $00584347,1.1089688364,0.9735667237 \backslash \mathrm{H}, 1.1933500563,1.2601384155,-0.7770$ $794139 \backslash \mathrm{C}, 3.7580668349,0.5784154428,0.0640221628 \backslash \mathrm{H}, 2.503491605,-1.02314$ $95115,0.7982783404 \backslash \mathrm{H}, 2.5158470264,-0.8721355664,-0.9512445835 \backslash \mathrm{H}, 4.6677$ $638152,-0.0311886567,0.0178134391 \backslash \mathrm{H}, 3.7984279929,1.2999247734,-0.76140$ $96028 \backslash \mathrm{H}, 3.7860316643,1.1478661725,1.0013066427 \backslash \backslash$ Version=IA $64 \mathrm{~L}-\mathrm{G} 03 \mathrm{RevD}$. $01 \backslash \mathrm{State}=2-\mathrm{A} \backslash \mathrm{HF}=-236.4127206 \backslash \mathrm{S} 2=0.753882 \backslash \mathrm{S} 2-1=0 . \backslash \mathrm{S} 2 \mathrm{~A}=0.75001 \backslash \mathrm{RMSD}=7.82$ $1 e-09 \backslash$ RMSF $=2.423 e-07 \backslash$ Thermal $=0 . \backslash$ Dipole $=0.0675146,0.0508499,0.0369449 \backslash P$ $\mathrm{G}=\mathrm{C} 01 \quad[\mathrm{X}(\mathrm{C} 6 \mathrm{H} 13)] \backslash \backslash @$ 


\section{$\mathrm{CH}_{2}=\mathrm{CHCl}$}

$1 \backslash 1 \backslash G I N C-S C 31 \backslash F O p t \backslash R B 3 L Y P \backslash 6-31 G(d) \backslash C 2 H 3 C 11 \backslash M L C 501 \backslash 29-O c t-2004 \backslash 0 \backslash \backslash \#$ B3L YP/6-31G* OPT FREQ MAXDISK $=65536000 \backslash \backslash \mathrm{VC}$ mon-CS-6dub3 $\backslash 0,1 \backslash \mathrm{H}, 1.62530240$ $26,2.0804916963,0 . \backslash \mathrm{C}, 1.3019223184,1.043655435,0 . \backslash \mathrm{C}, 0.0040520921,0.7657$ $383328,0 . \backslash \mathrm{H}, 2.0647178362,0.2720644443,0 . \backslash \mathrm{H},-0.7795192128,1.5150476964$, $0 . \backslash C 1,-0.6321380876,-0.8661156732,0 . \backslash \backslash$ Version=DEC-AXP-OSF/1-G03RevB.03 $\backslash$ State $=1-A \cdot \backslash H F=-538.1853947 \backslash R M S D=5.316 e-09 \backslash R M S F=1.887 e-05 \backslash D i p o l e=0.266$ $6289,0.5759036,0 . \backslash P G=C S[S G(C 2 H 3 C 11)] \backslash \backslash$

\section{$\mathrm{CH}_{3} \mathrm{CH}(\mathrm{Cl}) \bullet$}

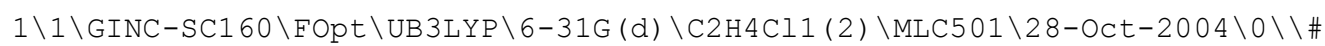

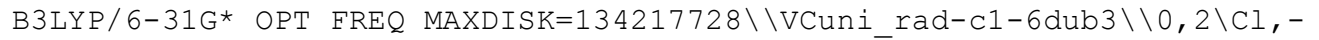
$0.9213473796,-0.1567571544,-0.6335833185 \backslash C, 0.2518145377,-0.3182418856$, $0.6364772502 \backslash \mathrm{C}, 1.4410501028,0.5718702802,0.6438684742 \backslash \mathrm{H}, 1.9774651662,0$ $.4624120607,1.5931459184 \backslash \mathrm{H}, 1.1560197405,1.624007444,0.5247202707 \backslash \mathrm{H}, 0.2$ $235551552,-1.2776388027,1.1392470656 \backslash \mathrm{H}, 2.1486775477,0.3343205543,-0.16$ $82711863 \backslash \backslash$ Version=DEC-AXP-OSF $/ 1-G 03 R e v B .03 \backslash$ State $=2-A \backslash H F=-538.7611943 \backslash \mathrm{S}$ $2=0.753938 \backslash \mathrm{S} 2-1=0 . \backslash \mathrm{S} 2 \mathrm{~A}=0.750011 \backslash \mathrm{RMSD}=6.952 \mathrm{e}-09 \backslash \mathrm{RMSF}=5.761 \mathrm{e}-05 \backslash \mathrm{Dipole}=0$ $.5558682,-0.0331324,0.3369899 \backslash \mathrm{PG}=\mathrm{C} 01 \quad[\mathrm{X}(\mathrm{C} 2 \mathrm{H} 4 \mathrm{Cl} 1)] \backslash \backslash$

\section{$\mathrm{CH3CH}(\mathrm{Cl}) \mathrm{CH} 2 \mathrm{CH}(\mathrm{Cl}) \bullet$}

$1 \backslash 1 \backslash G I N C-S C 160 \backslash F O p t \backslash U B 3 L Y P \backslash 6-31 G(d) \backslash C 4 H 7 C 12(2) \backslash M L C 501 \backslash 28-O c t-2004 \backslash 0 \backslash \backslash \#$

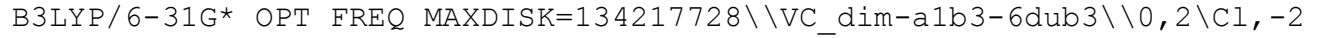
$.5248717104,0.0248857158,-0.4037613942 \backslash C,-1.3097747452,-0.1974371897,0$ $.8152474465 \backslash \mathrm{C},-0.1197784977,0.696531008,0.8219396367 \backslash \mathrm{C}, 0.923687349,0.4$ $298552262,-0.2821694203 \backslash \mathrm{C}, 2.0568006056,1.4463854063,-0.2836998546 \backslash \mathrm{H}, 1$. $6499307746,2.4485118462,-0.4674936907 \backslash \mathrm{H}, 2.5784749538,1.4556285455,0.67$ $88693013 \backslash \mathrm{H}, 2.7840547399,1.2211750756,-1.0682633191 \backslash \mathrm{H}, 0.4288211502,0.39$ $92185104,-1.2555237655 \backslash \mathrm{Cl}, 1.6211143001,-1.2572734045,-0.0721840198 \backslash \mathrm{H}, 0$ $.3742925177,0.6128375664,1.7981688856 \backslash \mathrm{H},-0.4435964746,1.7408291987,0.7$ $06617135 \backslash \mathrm{H},-1.3137099563,-1.17961674,1.2707906415 \backslash \backslash$ Version=DEC-AXP-OSF $/ 1-G 03 R e v B .03 \backslash$ State $=2-A \backslash H F=-1076.9865605 \backslash S 2=0.753923 \backslash \mathrm{S} 2-1=0 . \backslash \mathrm{S} 2 \mathrm{~A}=0.750$ $011 \backslash \mathrm{RMSD}=4.754 \mathrm{e}-09 \backslash \mathrm{RMSF}=6.451 \mathrm{e}-05 \backslash \mathrm{Dipole}=0.2186697,0.7607045,0.2092827$ $\backslash \mathrm{PG}=\mathrm{CO} \quad[\mathrm{X}(\mathrm{C} 4 \mathrm{H} 7 \mathrm{Cl})] \backslash \backslash$

\section{$\mathrm{CH}_{3} \mathrm{CH}(\mathrm{Cl}) \mathrm{CH}_{2} \mathrm{CH}(\mathrm{Cl}) \mathrm{CH}_{2} \mathrm{CH}(\mathrm{Cl}) \bullet$}

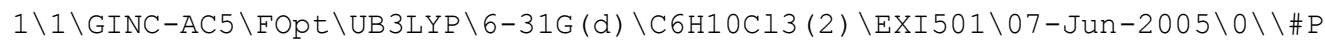
GFINPUT B3LYP $/ 6-31 \mathrm{G} * \mathrm{OPT}=(\mathrm{MAXCYC}=300) \quad$ INT $(\mathrm{GRID}=\mathrm{ULTRAFINE}) \quad$ MAXDISK $=134$ $217728 \operatorname{IOP}(1 / 8=10) \backslash \backslash \mathrm{opt}$ conf $\backslash \backslash 0,2 \backslash \mathrm{C},-1.6947034442,-0.3392514234,-2.30$ $26586661 \backslash \mathrm{C},-1.6974009183,-0.3226396782,-0.8140636398 \backslash \mathrm{C},-0.3066997165,-$ $0.3328023986,-0.1451841606 \backslash C,-0.3990305398,-0.2230374551,1.3764182494 \backslash$ C, $0.9279939984,-0.0973100379,2.1247942407 \backslash C, 0.7550177592,-0.121506554$, $3.6380668726 \backslash \mathrm{Cl}, 1.7647943129,1.4693007911,1.6419729098 \backslash \mathrm{H}, 1.6174591936$, $-0.8802165788,1.8038385866 \backslash \mathrm{H}, 0.3153161798,-1.0802322613,3.9397470183 \backslash \mathrm{H}$ $, 1.7181089319,-0.0109027258,4.1430742436 \backslash \mathrm{H}, 0.0938980582,0.6844038221,3$ $.9730275353 \backslash \mathrm{H},-0.9206392153,-1.1077834134,1.7657480225 \backslash \mathrm{H},-1.0214404095$ $, 0.6497588757,1.6150262892 \backslash \mathrm{Cl}, 0.5765533984,-1.8773600337,-0.6051916625$ $\backslash \mathrm{H}, 0.3097387594,0.4709596879,-0.5516119103 \backslash \mathrm{H},-2.2682869557,-1.18982947$ $02,-0.458292087 \backslash \mathrm{H},-2.2137368984,0.5803195915,-0.4583283728 \backslash \mathrm{H},-1.611293$ $6676,-1.2493148511,-2.8830479398 \backslash \mathrm{Cl},-1.2548881118,1.0993605724,-3.1634$ $529392 \backslash \backslash$ Version=IA64L-G03RevC.02 \State $=2-A \backslash H F=-1615.211402 \backslash \mathrm{S} 2=0.753937$ $\backslash \mathrm{S} 2-1=0 . \backslash \mathrm{S} 2 \mathrm{~A}=0.750011 \backslash \mathrm{RMSD}=8.850 \mathrm{e}-09 \backslash \mathrm{RMSF}=3.694 \mathrm{e}-05 \backslash \mathrm{Dipole}=-0.8469234$, $-0.4624166,0.8625457 \backslash \mathrm{PG}=\mathrm{C0} 1 \quad[\mathrm{X}(\mathrm{C} 6 \mathrm{H} 10 \mathrm{Cl3})] \backslash \backslash$

\section{$\mathrm{CH}_{2}=\mathrm{CHCN}$}

$1 \backslash 1 \backslash$ GINC-SC4 $0 \backslash F O p t \backslash R B 3 L Y P \backslash 6-31 G(d) \backslash C 3 H 3 N 1 \backslash M L C 501 \backslash 29-0 c t-2004 \backslash 0 \backslash \backslash \#$ B3LY

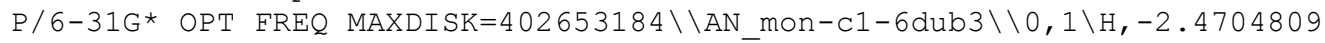
$703,-0.9133599665,-0.0005223479 \backslash C,-1.38 \overline{5} 4115495,-0.8925802502,-0.00055$ 
$51139 \backslash \mathrm{C},-0.7255359894,0.2720120984,-0.0005188656 \backslash \mathrm{H},-0.8662601887,-1.84$ $5888087,-0.0005470568 \backslash \mathrm{H},-1.2575486291,1.2204112267,-0.0006036754 \backslash \mathrm{C}, 0.7$ $030616653,0.3567006931,-0.000275243 \backslash N, 1.8630864329,0.4460059399,0.0013$ $954879 \backslash \backslash$ Version=DEC-AXP-OSF $/ 1-G 03$ RevB.03\State $=1-A \backslash H F=-170.8315503 \backslash \mathrm{RMS}$ $\mathrm{D}=5.028 \mathrm{e}-09 \backslash \mathrm{RMSF}=6.752 \mathrm{e}-05 \backslash \mathrm{Dipole}=-1.5058324,-0.2565732,-0.0011562 \backslash \mathrm{PG}=$ $\mathrm{C} 01[\mathrm{X}(\mathrm{C} 3 \mathrm{H} 3 \mathrm{~N} 1)] \backslash \backslash @$

\section{$\mathrm{CH}_{3} \mathrm{CH}(\mathrm{CN}) \bullet$}

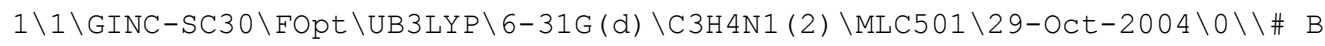

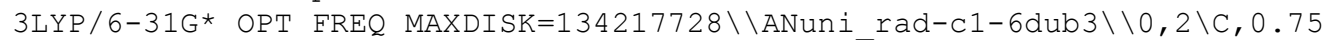
$43429042,-0.2983335115,0.3122792987 \backslash \mathrm{N}, 1.87376 \overline{3} 2074,-0.1694924694,0.651$ $2865892 \backslash C,-0.5657744262,-0.4432506421,-0.0889032139 \backslash \mathrm{C},-1.3947270475,0$. $6895274827,-0.6074519143 \backslash \mathrm{H},-1.0042180897,-1.435250357,-0.0169189576 \backslash \mathrm{H}$, $-2.2901219682,0.8309596994,0.0139675375 \backslash \mathrm{H},-0.8347741583,1.6274002584,-$ $0.6264006175 \backslash \mathrm{H},-1.7502768181,0.4756777102,-1.6251991097 \backslash \backslash$ Version=DEC $-\mathrm{A}$ $\mathrm{XP}-\mathrm{OSF} / 1-\mathrm{G} 03 \mathrm{RevB} .03 \backslash \mathrm{State}=2-\mathrm{A} \backslash \mathrm{HF}=-171.416282 \backslash \mathrm{S} 2=0.766962 \backslash \mathrm{S} 2-1=0 . \backslash \mathrm{S} 2 \mathrm{~A}=0$ $.750126 \backslash \mathrm{RMSD}=9.889 \mathrm{e}-09 \backslash \mathrm{RMSF}=1.080 \mathrm{e}-05 \backslash \mathrm{Dipole}=-1.4561102,-0.0162055,-0$. $4740771 \backslash \mathrm{PG}=\mathrm{C} 01 \quad[\mathrm{X}(\mathrm{C} 3 \mathrm{H} 4 \mathrm{~N} 1)] \backslash \backslash$

\section{$\mathrm{CH}_{3} \mathrm{CH}(\mathrm{CN}) \mathrm{CH}_{2} \mathrm{CH}(\mathrm{CN}) \bullet$}

$1 \backslash 1 \backslash G I N C-S C 59 \backslash F O p t \backslash U B 3 L Y P \backslash 6-31 G(d) \backslash C 6 H 7 N 2(2) \backslash M L C 501 \backslash 30-O c t-2004 \backslash 0 \backslash \backslash \# B$ $3 \mathrm{LYP} / 6-31 \mathrm{G}^{*} \mathrm{OPT}=(\mathrm{MAXCYC}=300) \quad \mathrm{MAXDISK}=402653184 \backslash \backslash$ ANdim_rad-a1b3-6dub3 $\backslash \backslash$ $0,2 \backslash \mathrm{C},-0.5300436751,0.2701347661,-1.2445521638 \backslash \mathrm{C},-0.8 \overline{0} 98252162,0.01285$ $50204,0.2062101572 \backslash \mathrm{C},-1.0987806681,-0.4844462004,-2.2592857071 \backslash \mathrm{H}, 0.146$ $4141302,1.0750475787,-1.5205411551 \backslash \mathrm{C}, 0.4671583274,-0.3064756239,1.0398$ $978863 \backslash \mathrm{H},-1.5062966777,-0.8237425312,0.3172868854 \backslash \mathrm{H},-1.2893780236,0.89$ $88614784,0.6481066193 \backslash \mathrm{H}, 0.9445244035,-1.1943339941,0.6055680908 \backslash \mathrm{C}, 1.43$ $32463279,0.7983314892,0.9365385193 \backslash \mathrm{C}, 0.120500673,-0.5944691949,2.51363$ $14124 \backslash \mathrm{H}, 1.0212401778,-0.83174342,3.0865882383 \backslash \mathrm{H},-0.5622722314,-1.44809$ $14387,2.5728073515 \backslash \mathrm{H},-0.3635145988,0.2709140463,2.9774789042 \backslash \mathrm{N},-1.5889$ $327565,-1.1317216823,-3.1109657491 \backslash \mathrm{N}, 2.176896786,1.6856512169,0.847832$ $0977 \backslash \backslash$ Version=DEC-AXP-OSF $/ 1-G 03 R e v B .03 \backslash$ State $=2-A \backslash H F=-342.280167 \backslash S 2=0.7$ $67193 \backslash \mathrm{S} 2-1=0 . \backslash \mathrm{S} 2 \mathrm{~A}=0.750128 \backslash \mathrm{RMSD}=4.101 \mathrm{e}-09 \backslash \mathrm{RMSF}=3.523 e-05 \backslash \mathrm{Dipole}=-0.421$ $0172,-0.393732,1.3398982 \backslash \mathrm{PG}=\mathrm{C} 01 \quad[\mathrm{X}(\mathrm{C} 6 \mathrm{H} 7 \mathrm{~N} 2)] \backslash \backslash \mathrm{C}$

\section{$\mathrm{CH}_{3} \mathrm{CH}(\mathrm{CN}) \mathrm{CH}_{2} \mathrm{CH}(\mathrm{CN}) \mathrm{CH}_{2} \mathrm{CH}(\mathrm{CN}) \bullet$}

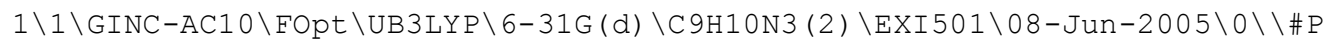
GFINPUT B3LYP/6-31G* OPT $=($ MAXCYC $=300)$ MAXDISK $=134217728$ MAXDISK $=13421$ $7728 \backslash \backslash$ opt confs $\backslash \backslash 0,2 \backslash C,-1.5357452848,0.2468758669,-2.3005866535 \backslash C,-1.5$ $370452314,0.2487858552,-0.800308516 \backslash C,-0.1091430762,0.250017538,-0.179$ $1929895 \backslash \mathrm{C},-0.1756531628,0.2376757635,1.3659429554 \backslash \mathrm{C}, 1.1988674079,0.140$ $2390804,2.0685837927 \backslash \mathrm{C}, 1.0592691595,0.2243407305,3.602018377 \backslash \mathrm{H}, 1.82473$ $18891,0.9756080324,1.7267930521 \backslash \mathrm{C}, 1.8843663063,-1.1021315186,1.6762823$ $756 \backslash \mathrm{H},-0.6715912173,1.1511224887,1.7145408859 \backslash \mathrm{H},-0.7979406357,-0.61080$ $3281,1.6734985903 \backslash \mathrm{H}, 0.5937669547,1.1758295515,3.8783303847 \backslash \mathrm{H}, 2.0378437$ $085,0.1654611128,4.0862981636 \backslash \mathrm{H}, 0.4377786971,-0.5917053263,3.984408080$ $2 \backslash \mathrm{N}, 2.3993043174,-2.0924708036,1.3563859782 \backslash \mathrm{H}, 0.4164035265,-0.65197634$ $78,-0.5186390892 \backslash \mathrm{C}, 0.6561302315,1.4131482864,-0.6542700005 \backslash \mathrm{H},-2.068729$ $7767,1.141868308,-0.4382404092 \backslash \mathrm{H},-2.0745697716,-0.6253786057,-0.420798$ $6997 \backslash \mathrm{H},-1.0001559674,1.0285144718,-2.8333096826 \backslash \mathrm{C},-2.2126866062,-0.707$ $5661382,-3.0438605985 \backslash \mathrm{N}, 1.2408986577,2.348715666,-1.0165149331 \backslash \mathrm{N},-2.79$ $27309565,-1.5229381751,-3.6628044355 \backslash \backslash$ Version=IA64L-G03RevC.02 State $=2$ $-\mathrm{A} \backslash \mathrm{HF}=-513.1437601 \backslash \mathrm{S} 2=0.767346 \backslash \mathrm{S} 2-1=0 . \backslash \mathrm{S} 2 \mathrm{~A}=0.75013 \backslash \mathrm{RMSD}=9.203 e-09 \backslash \mathrm{RMSF}$ $=3.191 e-06 \backslash$ Dipole $=-0.7476544,1.0168316,1.8439307 \backslash \mathrm{PG}=\mathrm{C} 01 \quad[\mathrm{X}(\mathrm{C} 9 \mathrm{H} 10 \mathrm{~N} 3)] \backslash \backslash$

\section{1-CH - -phosphetane}

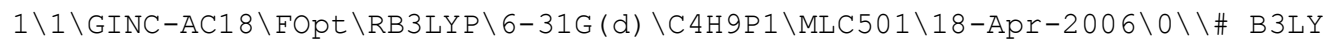

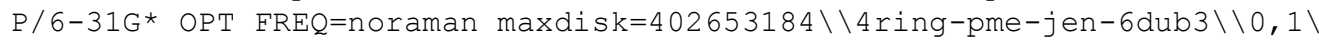
$\mathrm{P}, 0.7360220526,0.4417382522,0 . \backslash \mathrm{C},-0.2456863106,2.0299646568,0 . \backslash \mathrm{C},-0.41$ $80963389,-1.7082864288,0 . \backslash \mathrm{H},-1.329232766,1.8630016021,0 . \backslash \mathrm{H},-1.35496590$ $75,-2.2782703993,0 . \backslash \mathrm{H}, 0.4056757764,-2.4308000734,0 . \backslash \mathrm{C},-0.2455457277,-0$ $.6953968263,1.1616949048 \backslash \mathrm{C},-0.2455457277,-0.6953968263,-1.1616949048 \backslash \mathrm{H}$ $, 0.0201066393,2.6211800433,0.8837486017 \backslash \mathrm{H}, 0.0201066393,2.6211800433,-0$ 
$.8837486017 \backslash \mathrm{H},-1.2005538256,-0.2451412088,1.4554691283 \backslash \mathrm{H},-1.2005538256$ $,-0.2451412088,-1.4554691283 \backslash \mathrm{H}, 0.2643591612,-1.0583963129,2.0583003948$ $\backslash \mathrm{H}, 0.2643591612,-1.0583963129,-2.0583003948 \backslash \backslash$ Version=IA64L-G03RevD .01 State $=1-A^{\prime} \backslash \mathrm{HF}=-499.1753417 \backslash \mathrm{RMSD}=4.129 \mathrm{e}-09 \backslash \mathrm{RMSF}=1.685 \mathrm{e}-05 \backslash$ Thermal $=0 . \backslash \mathrm{Di}$ pole $=-0.5445568,0.0139908,0 . \backslash P G=C S[S G(C 2 H 3 P 1), X(C 2 H 6)] \backslash \backslash @$

\section{$\mathrm{CH}_{3} \mathrm{P}\left(\mathrm{CH}_{3}\right) \mathrm{CH}_{2} \mathrm{CH}_{2} \mathrm{CH}_{2} \bullet$}

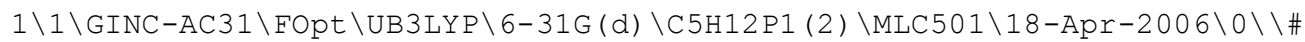

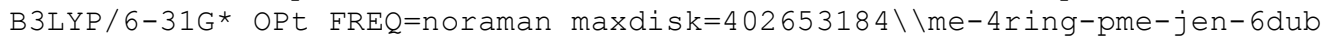
$3 \backslash \backslash 0,2 \backslash \mathrm{C}, 3.164642,-0.186987,0.25156 \backslash \mathrm{C}, 1.869688,0.299172,-0.309129 \backslash \mathrm{C}, 0$. $654862,-0.529849,0.188047 \backslash \mathrm{P},-0.971871,-0.006346,-0.592382 \backslash \mathrm{C},-2.115697$, $-1.158747,0.331148 \backslash \mathrm{H},-3.155838,-0.889429,0.117196 \backslash \mathrm{H},-1.962851,-1.12825$ $3,1.41726 \backslash \mathrm{H},-1.961496,-2.186178,-0.015824 \backslash \mathrm{C},-1.306713,1.564979,0.36247$ $1 \backslash \mathrm{H}, 0.588539,-0.482114,1.283965 \backslash \mathrm{H}, 0.808604,-1.584305,-0.076336 \backslash \mathrm{H}, 1.718$ $368,1.352647,-0.039415 \backslash \mathrm{H}, 1.88995,0.254839,-1.407008 \backslash \mathrm{H}, 3.548477,0.19673$ $8,1.192086 \backslash \mathrm{H}, 3.651788,-1.064073,-0.165165 \backslash \mathrm{H},-2.322805,1.911598,0.14439$ $4 \backslash \mathrm{H},-1.20881,1.42959,1.447146 \backslash \mathrm{H},-0.616556,2.352718,0.042847 \backslash \backslash$ Version $=\mathrm{I}$ $\mathrm{A} 64 \mathrm{~L}-\mathrm{G} 03 \mathrm{RevD} .01 \backslash \mathrm{State}=2-\mathrm{A} \backslash \mathrm{HF}=-539.0511121 \backslash \mathrm{S} 2=0.753887 \backslash \mathrm{S} 2-1=0 . \backslash \mathrm{S} 2 \mathrm{~A}=0.75$ $001 \backslash \mathrm{RMSD}=8.241 \mathrm{e}-09 \backslash \mathrm{RMSF}=1.132 \mathrm{e}-05 \backslash \mathrm{Thermal}=0 . \backslash \mathrm{Dipole}=-0.0709248,-0.0427$ $925,0.5302575 \backslash \mathrm{PG}=\mathrm{C} 01 \quad[\mathrm{X}(\mathrm{C} 5 \mathrm{H} 12 \mathrm{P} 1)] \backslash \backslash @$

\section{$\mathrm{CH}_{3} \mathrm{CH}_{2} \mathrm{CH}_{2} \mathrm{P}\left(\mathrm{CH}_{3}\right) \mathrm{CH}_{2} \mathrm{CH}_{2} \mathrm{CH}_{2} \bullet$}

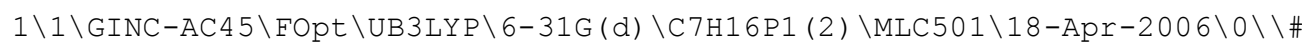
B3LYP/6-31G* Opt=tight INT (grid=ultrafine) $F R E Q=$ noraman maxdisk=402653 $184 \backslash \backslash$ npropyl-4ring-c1-6dub3\\0,2\C,3.6047277798,-0.4490393315, 0.242383 $3832 \backslash \mathrm{C}, 2.3661942396,0.1622773001,-0.3232058604 \backslash \mathrm{C}, 1.0724006047,-0.52874$ $85782,0.1866838694 \backslash \mathrm{P},-0.4941750346,0.1692371981,-0.5827401048 \backslash \mathrm{C},-1.747$ $5670461,-0.8676215414,0.3547049773 \backslash \mathrm{C},-3.2072671313,-0.5235209061,0.020$ $4757462 \backslash \mathrm{H},-1.5765867412,-0.7826079051,1.4381560001 \backslash \mathrm{H},-1.5516592189,-1$. $9151604031,0.0860017811 \backslash \mathrm{C},-0.629774144,1.7741754886,0.3664189679 \backslash \mathrm{H}, 1.0$ $227943053,-0.4711906677,1.2830047962 \backslash \mathrm{H}, 1.108779508,-1.5943051237,-0.07$ $49594838 \backslash \mathrm{H}, 2.3268514737,1.2290773117,-0.0665568187 \backslash \mathrm{H}, 2.3786946656,0.10$ $24782181,-1.4203269869 \backslash \mathrm{H}, 4.0215242418,-0.1021399925,1.183249202 \backslash \mathrm{H}, 4.00$ $34553071,-1.3717716879,-0.169559153 \backslash \mathrm{H},-1.5920833638,2.2501681469,0.150$ $9991672 \backslash \mathrm{H},-0.5437509912,1.6314630637,1.4512803714 \backslash \mathrm{H}, 0.1542668284,2.465$ $4654013,0.0402188475 \backslash \mathrm{C},-4.2103034705,-1.4286734533,0.7454049101 \backslash \mathrm{H},-3.4$ $115756688,0.5229835579,0.2839703919 \backslash \mathrm{H},-3.359207055,-0.6006940844,-1.06$ $41729339 \backslash \mathrm{H},-5.2428331966,-1.1632812271,0.4901599167 \backslash \mathrm{H},-4.059582463,-2$. $4812442481,0.4759068353 \backslash \mathrm{H},-4.1039634289,-1.3473665363,1.8341621781 \backslash \backslash \mathrm{Ve}$ rsion $=I A 64 \mathrm{~L}-\mathrm{G} 03$ RevD.01 $\backslash$ State $=2-\mathrm{A} \backslash \mathrm{HF}=-617.676619 \backslash \mathrm{S} 2=0.753887 \backslash \mathrm{S} 2-1=0 . \backslash \mathrm{S} 2$ $\mathrm{A}=0.75001 \backslash \mathrm{RMSD}=8.814 \mathrm{e}-09 \backslash \mathrm{RMSF}=8.563 \mathrm{e}-07 \backslash \mathrm{Thermal}=0 . \backslash \mathrm{Dipole}=-0.0984401$, $0.0407625,0.5152411 \backslash \mathrm{PG}=\mathrm{C} 01 \quad[\mathrm{X}(\mathrm{C} 7 \mathrm{H} 16 \mathrm{P} 1)] \backslash \backslash \mathrm{e}$

\section{$\left(\left(\mathrm{CH}_{3}\right)_{3} \mathrm{Si}\right)_{3} \mathrm{SiH}$}

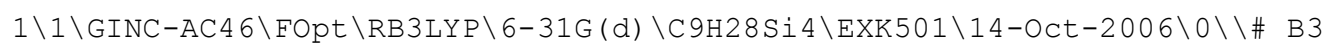
LYP/6-31G* OPT FREQ=NORAMAN MAXDISK=134217728\\H-Si-SiMe3 a new $\backslash \backslash 0,1 \backslash S$ $i,-2.1960858362,-0.2591538367,0.5581971049 \backslash \mathrm{Si}, 0.177717037 \overline{6},-0.21591111$ $21,0.6223704525 \backslash \mathrm{Si}, 1.0597414953,1.9806589009,0.4245660487 \backslash \mathrm{H}, 0.56438816$ $3,-0.6948963478,1.9965100283 \backslash \mathrm{Si}, 1.1607430611,-1.7499459026,-0.90169750$ $16 \backslash \mathrm{C},-2.8232897701,-0.1262700647,-1.2342112453 \backslash \mathrm{C},-2.8366516107,-1.8813$ $839454,1.3175655002 \backslash \mathrm{C},-2.9294165503,1.188458228,1.5531305883 \backslash \mathrm{C}, 0.31028$ $15113,2.8718079185,-1.0809856223 \backslash \mathrm{C}, 0.6571166292,2.9812654486,1.9913483$ $283 \backslash C, 2.9514590824,1.9389995926,0.2179018338 \backslash C, 1.1165543733,-1.0612639$ $008,-2.675405128 \backslash \mathrm{C}, 2.9711307426,-2.0772684851,-0.4185440566 \backslash \mathrm{C}, 0.235927$ $3158,-3.4133584673,-0.8831588532 \backslash \mathrm{H},-3.9206770573,-0.1224867504,-1.2590$ $289872 \backslash \mathrm{H},-2.4788822983,0.7964411184,-1.716016535 \backslash \mathrm{H},-2.4828655027,-0.96$ $80284802,-1.8491231861 \backslash \mathrm{H}, 0.7253812329,3.8832604704,-1.1785054511 \backslash \mathrm{H}, 0.5$ $195815771,2.3365850363,-2.0146693665 \backslash \mathrm{H},-0.7781859203,2.9701237744,-0.9$ $922178037 \backslash \mathrm{H}, 1.5598347888,-1.7786044568,-3.3781281205 \backslash \mathrm{H}, 0.0911944507,-0$ $.8621206355,-3.0086430107 \backslash \mathrm{H}, 1.6805293777,-0.1248913314,-2.7613195477 \backslash \mathrm{H}$ $,-4.0257694887,1.1313989819,1.5607716783 \backslash \mathrm{H},-2.5894456753,1.1763177628$, $2.5952366234 \backslash \mathrm{H},-2.65263185,2.1587956071,1.1246306916 \backslash \mathrm{H}, 0.7270592111,-4$ $.1322238076,-1.5519886084 \backslash \mathrm{H}, 0.2192788002,-3.8528243218,0.1210353272 \backslash \mathrm{H}$, $-0.8027521845,-3.3079026669,-1.2172574781 \backslash H, 3.3573119162,2.9585798462$, 
$0.1863859735 \backslash \mathrm{H}, 3.4355707351,1.4151103768,1.0502816317 \backslash \mathrm{H}, 3.2497751671,1$ $.4368626315,-0.7098648664 \backslash \mathrm{H},-3.9342214705,-1.9045129194,1.313587811 \backslash \mathrm{H}$, $-2.4831539791,-2.7587654972,0.7642283173 \backslash \mathrm{H},-2.505223706,-1.9894360892$, $2.3568364683 \backslash \mathrm{H}, 3.425365433,-2.8101419516,-1.0980465479 \backslash \mathrm{H}, 3.5751362007$, $-1.1638148289,-0.4612135137 \backslash \mathrm{H}, 3.0454013991,-2.4757645032,0.5998169117 \backslash$ $\mathrm{H}, 1.0825895092,3.9911335661,1.9254031456 \backslash \mathrm{H},-0.423698244,3.0837265395,2$ $.1406629898 \backslash \mathrm{H}, 1.0708184635,2.5030882364,2.886681891 \backslash \backslash$ Version=IA64L-G03 RevC.02 $\backslash$ State $=1-A \backslash H F=-1517.9165474 \backslash R M S D=3.795 e-09 \backslash R M S F=7.031 e-05 \backslash D i p o l$ $e=-0.0610392,0.0761043,-0.2134493 \backslash P G=C 01 \quad[X(C 9 H 28 S i 4)] \backslash \backslash @$

\section{$\left(\left(\mathrm{CH}_{3}\right)_{3} \mathrm{Si}\right)_{3} \mathrm{Si} \bullet$}

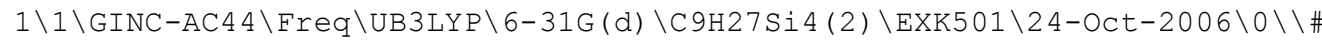
B3LYP /6-31G* FREQ=NORAMAN MAXDISK $=134217728 \backslash \backslash \mathrm{Si}-\mathrm{SiMe}-\mathrm{rad}$ new_sp $\backslash \backslash 0,2$ $\backslash \mathrm{Si},-2.2807328686,-0.0250106715,0 . \backslash \mathrm{Si}, 0.0573981314,-0.3751 \overline{5} 667 \overline{1} 5,0 . \backslash \mathrm{Si}$ $, 1.1523911314,-0.0226546715,2.066058 \backslash \mathrm{Si}, 1.1523911314,-0.0226546715,-2$. $066058 \backslash C,-2.6938528686,1.8346583285,0 . \backslash C,-3.0694798686,-0.8081126715,-$ $1.543924 \backslash C,-3.0694798686,-0.8081126715,1.543924 \backslash C, 0.7649521314,1.71138$ $63285,2.753291 \backslash \mathrm{C}, 0.5550261314,-1.3123526715,3.330548 \backslash \mathrm{C}, 3.0388191314,-0$ $.1917216715,1.901454 \backslash \mathrm{C}, 0.7649521314,1.7113863285,-2.753291 \backslash \mathrm{C}, 3.0388191$ $314,-0.1917216715,-1.901454 \backslash \mathrm{C}, 0.5550261314,-1.3123526715,-3.330548 \backslash \mathrm{H},-$ $3.7810938686,1.9894473285,0 . \backslash \mathrm{H},-2.2839788686,2.3363823285,0.884233 \backslash \mathrm{H},-$ $2.2839788686,2.3363823285,-0.884233 \backslash \mathrm{H}, 1.2528381314,1.8556273285,3.7265$ $37 \backslash \mathrm{H}, 1.1217451314,2.4990073285,2.079926 \backslash \mathrm{H},-0.3117758686,1.8600483285,2$ $.896263 \backslash \mathrm{H}, 1.2528381314,1.8556273285,-3.726537 \backslash \mathrm{H},-0.3117758686,1.860048$ $3285,-2.896263 \backslash \mathrm{H}, 1.1217451314,2.4990073285,-2.079926 \backslash \mathrm{H},-4.1579208686,-$ $0.6652056715,1.531486 \backslash \mathrm{H},-2.8740678686,-1.8851486715,1.595364 \backslash \mathrm{H},-2.6881$ $498686,-0.3558116715,2.466825 \backslash \mathrm{H}, 1.0373611314,-1.1462306715,-4.302836 \backslash \mathrm{H}$ $, 0.7985571314,-2.3303906715,-3.005913 \backslash \mathrm{H},-0.5288598686,-1.2640726715,-3$ $.483408 \backslash \mathrm{H}, 3.5109591314,-0.0791506715,2.886242 \backslash \mathrm{H}, 3.3255141314,-1.172436$ $6715,1.505465 \backslash \mathrm{H}, 3.4638461314,0.5718243285,1.240831 \backslash \mathrm{H},-4.1579208686,-0$. $6652056715,-1.531486 \backslash \mathrm{H},-2.6881498686,-0.3558116715,-2.466825 \backslash \mathrm{H},-2.8740$ $678686,-1.8851486715,-1.595364 \backslash \mathrm{H}, 3.5109591314,-0.0791506715,-2.886242 \backslash$ $\mathrm{H}, 3.4638461314,0.5718243285,-1.240831 \backslash \mathrm{H}, 3.3255141314,-1.1724366715,-1$. $505465 \backslash \mathrm{H}, 1.0373611314,-1.1462306715,4.302836 \backslash \mathrm{H},-0.5288598686,-1.264072$ $6715,3.483408 \backslash \mathrm{H}, 0.7985571314,-2.3303906715,3.005913 \backslash \backslash$ Version=IA $64 \mathrm{~L}-\mathrm{G} 03$ RevC.02 $\backslash$ State $=2-A^{\prime} \backslash H F=-1517.2802122 \backslash S 2=0.75381 \backslash S 2-1=0 . \backslash S 2 A=0.750007 \backslash R M$ $\mathrm{SD}=5.808 \mathrm{e}-09 \backslash \mathrm{RMSF}=7.615 \mathrm{e}-06 \backslash \mathrm{Dipole}=0.017507,0.1266184,0 . \backslash \mathrm{PG}=\mathrm{CS}[\mathrm{SG}(\mathrm{C} 1 \mathrm{H} 1$ $\mathrm{Si2}), \mathrm{X}(\mathrm{C} 8 \mathrm{H} 26 \mathrm{Si2})] \backslash \backslash \mathrm{Q} \backslash$

\section{$\left(\mathrm{CH}_{3} \mathrm{CH}_{2}\right)_{3} \mathrm{SiH}$}

$1 \backslash 1 \backslash G I N C-A C 47 \backslash F O p t \backslash R B 3 L Y P \backslash 6-31 G(d) \backslash C 6 H 16$ Si $1 \backslash E X K 501 \backslash 13-F e b-2007 \backslash 0 \backslash \backslash \#$ B3

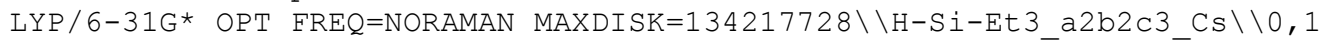
$\backslash \mathrm{H}, 0 ., 0 .,-1.7453111062 \backslash \mathrm{Si}, 0 ., 0 .,-0.2461583107 \backslash \mathrm{C}, 1.80654 \overline{5} 3877,0.0249766$ $572,0.3485801007 \backslash C,-0.9249031135,1.5520258703,0.3485801007 \backslash C,-0.881642$ $2742,-1.5770025275,0.3485801007 \backslash C, 2.6427865322,1.2128997,-0.1607168124$ $\backslash C,-2.3717952185,1.6822704237,-0.1607168124 \backslash C,-0.2709913137,-2.8951701$ $237,-0.1607168124 \backslash \mathrm{H}, 2.2819951914,-0.9160403472,0.038316319 \backslash \mathrm{H},-0.347683$ $3841,2.4342859807,0.038316319 \backslash \mathrm{H},-1.9343118073,-1.5182456334,0.03831631$ $9 \backslash \mathrm{H}, 1.813277083,0.0100481666,1.4483298312 \backslash \mathrm{H},-0.915340509,1.5653199346$, $1.4483298312 \backslash \mathrm{H},-0.8979365739,-1.5753681012,1.4483298312 \backslash \mathrm{H}, 2.6713501183$ $, 1.2412834897,-1.2564981116 \backslash \mathrm{H},-2.4106580945,1.69281532,-1.2564981116 \backslash \mathrm{H}$ $,-0.2606920238,-2.9340988097,-1.2564981116 \backslash \mathrm{H}, 3.6798185175,1.1573609107$ $, 0.1929611869 \backslash \mathrm{H},-2.8422132088,2.6081358621,0.1929611869 \backslash \mathrm{H},-0.837605308$ $7,-3.7654967729,0.1929611869 \backslash \mathrm{H}, 2.234474982,2.171691285,0.1802201968 \backslash \mathrm{H}$, $-2.997977313,0.8492664561,0.1802201968 \backslash \mathrm{H}, 0.763502331,-3.020957741,0.18$ $02201968 \backslash \backslash$ Version=IA64L-G03RevC.02\State $=1-A \backslash H F=-527.7931168 \backslash R M S D=2.08$ $7 e-09 \backslash \mathrm{RMSF}=1.200 e-05 \backslash \mathrm{Dipole}=0 ., 0 ., 0.2567041 \backslash \mathrm{PG}=\mathrm{C} 03 \quad[\mathrm{C} 3(\mathrm{Si} H \mathrm{H}), \mathrm{X}(\mathrm{C} 6 \mathrm{H} 15)$ ]$\backslash \backslash 0$

\section{$\left(\mathrm{CH}_{3} \mathrm{CH}_{2}\right)_{3} \mathrm{Si} \bullet$}

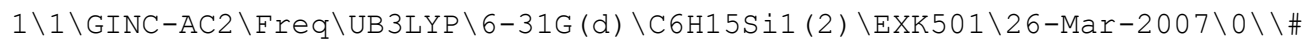
B3LYP/6-31G (D) FREQ=NORAMAN MAXDISK=134217728\\Si-Et3-c3-6dub3 B3LYP f req $\backslash \backslash 0,2 \backslash \mathrm{Si}, 0 ., 0 ., 0.3095569846 \backslash \mathrm{C}, 0.6866491077,1.6780341231,-0 . \overline{3} 01941 \overline{0} 1$ $54 \backslash \mathrm{C}, 1.1098951077,-1.4336728769,-0.3019410154 \backslash \mathrm{C},-1.7965448923,-0.24436$ 
$08769,-0.3019410154 \backslash C, 2.1169571077,2.0119181231,0.1540519846 \backslash C, 0.68389$ $41077,-2.8392978769,0.1540519846 \backslash \mathrm{C},-2.8008508923,0.8273791231,0.154051$ $9846 \backslash \mathrm{H}, 2.1885521077,2.0533121231,1.2472299846 \backslash \mathrm{H}, 0.6839451077,-2.921997$ $8769,1.2472299846 \backslash \mathrm{H},-2.8724968923,0.8686851231,1.2472299846 \backslash \mathrm{H}, 0.637917$ $1077,1.6782131231,-1.4029780154 \backslash \mathrm{H}, 1.1344171077,-1.3915588769,-1.402978$ $0154 \backslash \mathrm{H},-1.7723338923,-0.2866548769,-1.4029780154 \backslash \mathrm{H}, 0.0000001077,2.4698$ $971231,0.0257239846 \backslash \mathrm{H}, 2.1389941077,-1.2349488769,0.0257239846 \backslash \mathrm{H},-2.138$ $9938923,-1.2349488769,0.0257239846 \backslash \mathrm{H}, 2.4461841077,2.9835621231,-0.2348$ $030154 \backslash \mathrm{H}, 1.3607481077,-3.6102388769,-0.2348030154 \backslash \mathrm{H},-3.8069328923,0.62$ $66771231,-0.2348030154 \backslash \mathrm{H},-0.3259678923,-3.0883588769,-0.1924380154 \backslash \mathrm{H},-$ $2.5116138923,1.8264771231,-0.1924380154 \backslash \mathrm{H}, 2.8375821077,1.2618831231,-0$ $.1924380154 \backslash \backslash$ Version=IA 64L-G03RevC.02\State $=2-A \backslash H F=-527.1398414 \backslash S 2=0.7$ $51205 \backslash \mathrm{S} 2-1=0 . \backslash \mathrm{S} 2 \mathrm{~A}=0.750001 \backslash \mathrm{RMSD}=3.551 \mathrm{e}-09 \backslash \mathrm{RMSF}=3.479 \mathrm{e}-05 \backslash \mathrm{Dipole}=0.0000$ $405,0.0002671,-0.2746659 \backslash \mathrm{PG}=\mathrm{C} 01 \quad[\mathrm{X}(\mathrm{C} 6 \mathrm{H} 15 \mathrm{Si1})] \backslash \backslash @$

\section{$\left(\mathrm{CH}_{3}\right)_{2} \mathrm{CH}(\mathrm{CN})$}

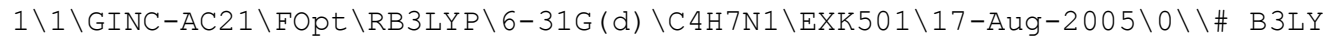

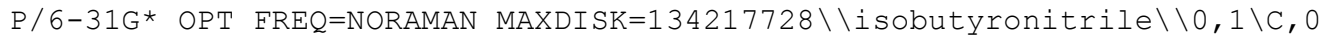
$.4711254125,0.2570409158,0 . \backslash \mathrm{C},-0.1503975154,-1.0777942439,0 . \backslash \mathrm{N},-0.6517$ $665487,-2.1251387935,0 . \backslash \mathrm{C}, 0.0911731686,1.0285631717,1.2797042977 \backslash \mathrm{C}, 0.0$ $911731686,1.0285631717,-1.2797042977 \backslash \mathrm{H}, 0.3820466055,0.476681511,2.1783$ $916316 \backslash \mathrm{H}, 0.3820466055,0.476681511,-2.1783916316 \backslash \mathrm{H},-0.9886570337,1.2051$ $154446,1.319918013 \backslash \mathrm{H},-0.9886570337,1.2051154446,-1.319918013 \backslash \mathrm{H}, 0.59954$ $68706,1.9984384464,1.2883644367 \backslash \mathrm{H}, 0.5995468706,1.9984384464,-1.2883644$ $367 \backslash \mathrm{H}, 1.5580475511,0.0972626596,0$. \\Version=IA64L-G03RevC.02 $\backslash$ State=1-A $\cdot \backslash \mathrm{HF}=-211.3831079 \backslash \mathrm{RMSD}=7.492 \mathrm{e}-09 \backslash \mathrm{RMSF}=1.152 \mathrm{e}-05 \backslash \mathrm{Dipole}=0.6079888,1.427$ $1339,0 . \backslash \mathrm{PG}=\mathrm{CS} \quad[\mathrm{SG}(\mathrm{C} 2 \mathrm{H} 1 \mathrm{~N} 1), \mathrm{X}(\mathrm{C} 2 \mathrm{H} 6)] \backslash \backslash \mathrm{e}$

\section{$\left(\mathrm{CH}_{3}\right)_{2} \mathrm{C}(\mathrm{CN}) \bullet$}

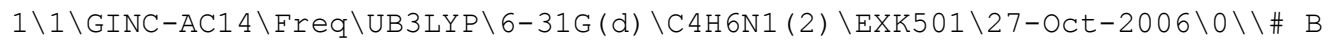

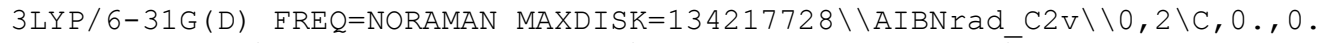
$,-0.308984027 \backslash \mathrm{C}, 0 ., 0 ., 1.082748973 \backslash \mathrm{N}, 0 ., 0 ., 2.259414973 \backslash \mathrm{C}, 0 ., 1.297207,-1$ $.065251027 \backslash \mathrm{C}, 0 .,-1.297207,-1.065251027 \backslash \mathrm{H}, 0 ., 2.162638,-0.398587027 \backslash \mathrm{H}, 0$. $,-2.162638,-0.398587027 \backslash \mathrm{H}, 0.881395,1.364093,-1.719577027 \backslash \mathrm{H},-0.881395,1$ $.364093,-1.719577027 \backslash \mathrm{H},-0.881395,-1.364093,-1.719577027 \backslash \mathrm{H}, 0.881395,-1$. $364093,-1.719577027 \backslash \backslash$ Version=IA $64 \mathrm{~L}-\mathrm{G} 03 \mathrm{RevC} .02 \backslash$ State $=2-\mathrm{B} 1 \backslash \mathrm{HF}=-210.73732$ $25 \backslash \mathrm{S} 2=0.76567 \backslash \mathrm{S} 2-1=0 . \backslash \mathrm{S} 2 \mathrm{~A}=0.750113 \backslash \mathrm{RMSD}=4.322 \mathrm{e}-09 \backslash \mathrm{RMSF}=1.702 \mathrm{e}-06 \backslash \mathrm{Dipol}$ $\mathrm{e}=0 ., 0 .,-1.6235724 \backslash \mathrm{PG}=\mathrm{C} 02 \mathrm{~V} \quad[\mathrm{C} 2(\mathrm{C} 1 \mathrm{C} 1 \mathrm{~N} 1), \mathrm{SGV}(\mathrm{C} 2 \mathrm{H} 2), \mathrm{X}(\mathrm{H} 4)] \backslash \backslash \mathrm{e}$

\section{Adamantane}

$1 \backslash 1 \backslash G I N C-A C 24 \backslash F O p t \backslash R B 3 L Y P \backslash 6-31 G(d) \backslash C 10 H 16 \backslash E X K 501 \backslash 12-O c t-2006 \backslash 0 \backslash \backslash \#$ B3LY $\mathrm{P} / 6-31 \mathrm{G} *$ OPT $\mathrm{FREQ}=\mathrm{NORAMAN}$ MAXDISK=268435456\\adamantane_new $\backslash \backslash 0,1 \backslash \mathrm{H},-0$. $0004344359,0 ., 2.6442443957 \backslash C,-0.0001803118,0 ., 1.5459029 \overline{4} 22 \backslash C,-1.458152$ $4429,0 .,-0.5159498712 \backslash \mathrm{C}, 0.7291501582,1.2623972721,-0.5151492501 \backslash \mathrm{C},-0.7$ $272832998,1.259665221,-1.0280111647 \backslash \mathrm{C}, 0.727493282,1.259452065,1.028423$ $5347 \backslash \mathrm{C},-1.4546553959,0 ., 1.0275096498 \backslash \mathrm{H},-1.249193633,2.1638761474,-0.68$ $43216678 \backslash \mathrm{H}, 0.2304119687,2.1639973033,1.406079376 \backslash \mathrm{H},-1.9895414545,0.882$ $4639002,1.4059745995 \backslash \mathrm{H},-2.4936816676,-0.0000000001,-0.8819749369 \backslash \mathrm{H}, 1.2$ $466585453,2.1591899095,-0.8815866492 \backslash \mathrm{H},-0.7404471822,1.2826292246,-2.1$ $267813113 \backslash \mathrm{H}, 1.7592892075,1.2810292066,1.4064529675 \backslash \mathrm{H},-1.9895414544,-0$. $8824639003,1.4059745995 \backslash \mathrm{C}, 1.4544036501,0 .,-1.0281829307 \backslash \mathrm{H}, 2.4988477024$ $, 0.0000000001,-0.6857083993 \backslash \mathrm{H}, 1.4800988135,0 .,-2.1270318681 \backslash \mathrm{C}, 0.727493$ $2821,-1.259452065,1.0284235347 \backslash \mathrm{H}, 0.2304119688,-2.1639973033,1.40607937$ $6 \backslash \mathrm{H}, 1.7592892076,-1.2810292065,1.4064529675 \backslash \mathrm{C},-0.7272832997,-1.2596652$ $211,-1.0280111647 \backslash \mathrm{H},-1.2491936329,-2.1638761475,-0.6843216678 \backslash \mathrm{H},-0.740$ $4471821,-1.2826292246,-2.1267813113 \backslash \mathrm{C}, 0.7291501582,-1.2623972721,-0.51$ $51492501 \backslash \mathrm{H}, 1.2466585454,-2.1591899094,-0.8815866492 \backslash \backslash$ Version=IA64L-G03 RevC.02\State $=1-A^{\prime} \backslash H F=-390.7252613 \backslash R M S D=1.597 e-09 \backslash R M S F=3.105 e-05 \backslash D i p o l$ $e=-0.000056,0 .,-0.0000773 \backslash P G=C S \quad[S G(C 4 H 4), X(C 6 H 12)] \backslash \backslash @$

\section{Adamantyl•}

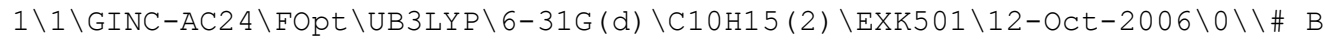
3LYP/6-31G* OPT FREQ=NORAMAN MAXDISK=268435456\\adamantyl_rad_new $\backslash \backslash 0,2$ 
$\backslash C,-0.000054611,0 ., 1.4889646692 \backslash C,-1.4631854391,0 \ldots,-0.4818283901 \backslash C, 0.7$ $316275575,1.2665753275,-0.4811721427 \backslash C,-0.7285271824,1.2615225583,-0.9$ $865633137 \backslash \mathrm{C}, 0.7255546938,1.2561542414,1.0816754884 \backslash \mathrm{C},-1.4508536435,0 .$, $1.0808563664 \backslash \mathrm{H},-1.2497604291,2.1649709777,-0.6402199607 \backslash \mathrm{H}, 0.2219250523$ $, 2.1576218524,1.4562840868 \backslash \mathrm{H},-1.9797302091,0.8866155606,1.4563393334 \backslash \mathrm{H}$ $,-2.4997672403,0 .,-0.8461209772 \backslash \mathrm{H}, 1.2495842636,2.1642844981,-0.8459567$ $692 \backslash \mathrm{H},-0.7438578944,1.2880637437,-2.0851800584 \backslash \mathrm{H}, 1.7580146697,1.270453$ $4286,1.4565140425 \backslash \mathrm{H},-1.9797302091,-0.8866155607,1.4563393334 \backslash \mathrm{C}, 1.45678$ $92444,0 .,-0.9864561991 \backslash \mathrm{H}, 2.5000706171,0 .,-0.6408013434 \backslash \mathrm{H}, 1.4873115848$, $0 .,-2.0851380906 \backslash \mathrm{C}, 0.7255546939,-1.2561542414,1.0816754884 \backslash \mathrm{H}, 0.2219250$ $523,-2.1576218524,1.4562840868 \backslash \mathrm{H}, 1.7580146698,-1.2704534286,1.45651404$ $25 \backslash \mathrm{C},-0.7285271824,-1.2615225584,-0.9865633137 \backslash \mathrm{H},-1.249760429,-2.16497$ $09777,-0.6402199607 \backslash \mathrm{H},-0.7438578944,-1.2880637437,-2.0851800584 \backslash \mathrm{C}, 0.73$ $16275575,-1.2665753275,-0.4811721427 \backslash \mathrm{H}, 1.2495842637,-2.164284498,-0.84$ $59567692 \backslash \backslash$ Version=IA64L-G03RevC.02 $\backslash$ State $=2-A^{\prime} \backslash \mathrm{HF}=-390.0585021 \backslash \mathrm{S} 2=0.753$ $445 \backslash \mathrm{S} 2-1=0 . \backslash \mathrm{S} 2 \mathrm{~A}=0.750009 \backslash \mathrm{RMSD}=4.256 \mathrm{e}-09 \backslash \mathrm{RMSF}=3.042 \mathrm{e}-05 \backslash \mathrm{Dipole}=0.000152$ $3,0 .,-0.3044914 \backslash P G=C S \quad[S G(C 4 H 3), X(C 6 H 12)] \backslash \backslash @$

\section{$\left(\mathbf{H}_{3} \mathrm{Si}\right)_{3} \mathrm{SiH}$}

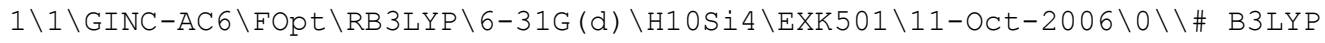
/6-31G* OPT FREQ=NORAMAN MAXDISK=268435456\\H-Si-SiH3_new $\backslash \backslash 0,1 \backslash \mathrm{Si},-2.2$ $426611773,0 .,-0.1649231005 \backslash \mathrm{Si}, 0.0002020332,0 ., 0.56062 \overline{9} 1254 \backslash \mathrm{Si}, 1.121249$ $9952,1.9424177035,-0.1649792869 \backslash \mathrm{H}, 0.0004465157,0 ., 2.0563369125 \backslash \mathrm{Si}, 1.12$ $12499952,-1.9424177035,-0.1649792869 \backslash \mathrm{H},-2.3028918295,0 .,-1.6531278537 \backslash$ $\mathrm{H},-2.9596125906,-1.2079076989,0.3303571275 \backslash \mathrm{H},-2.9596125906,1.207907698$ $9,0.3303571275 \backslash \mathrm{H}, 1.1516195899,1.9950034666,-1.6531168215 \backslash \mathrm{H}, 0.433100133$ $8,3.1668399264,0.3306117135 \backslash \mathrm{H}, 2.5258295999,1.9595043575,0.3303112936 \backslash \mathrm{H}$ $, 1.1516195899,-1.9950034666,-1.6531168215 \backslash \mathrm{H}, 2.5258295999,-1.9595043575$ $, 0.3303112936 \backslash \mathrm{H}, 0.4331001338,-3.1668399264,0.3306117135 \backslash \backslash$ Version=IA64L - G03RevC.02 \State $=1-A^{\prime} \backslash H F=-1163.9843138 \backslash R M S D=2.544 e-09 \backslash R M S F=3.741 e-06 \backslash$ Dipole $=-0.0000535,0 ., 0.037095 \backslash P G=C S \quad[S G(H 2 S i 2), X(H 8 S i 2)] \backslash \backslash @$

\section{$\left(\mathrm{H}_{3} \mathrm{Si}\right)_{3} \mathrm{Si} \bullet$}

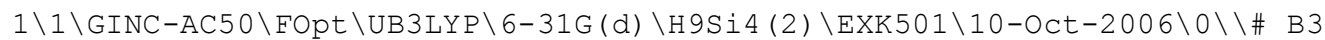
LYP /6-31G* OPT FREQ=NORAMAN MAXDISK=268435456\\Si-SiH3-rad new $\backslash \backslash 0,2 \backslash \mathrm{Si}$ $,-2.2989361994,0 .,-0.0865894244 \backslash \mathrm{Si},-0.0000681961,0 ., 0.3757 \overline{1} 9875 \backslash \mathrm{Si}, 1.1$ $494964828,1.9909663606,-0.0865001084 \backslash \mathrm{Si}, 1.1494964828,-1.9909663606,-0$. $0865001084 \backslash \mathrm{H},-2.5844846233,0 .,-1.5509941731 \backslash \mathrm{H},-2.9344270615,-1.2099697$ $245,0.5041851702 \backslash \mathrm{H},-2.9344270615,1.2099697245,0.5041851702 \backslash \mathrm{H}, 1.2920818$ $945,2.2391282395,-1.5507747008 \backslash \mathrm{H}, 0.4195369076,3.1460151713,0.505012511$ $2 \backslash \mathrm{H}, 2.515130581,1.9355841858,0.5041624697 \backslash \mathrm{H}, 1.2920818945,-2.2391282395$ $,-1.5507747008 \backslash \mathrm{H}, 2.515130581,-1.9355841858,0.5041624697 \backslash \mathrm{H}, 0.4195369076$ $,-3.1460151713,0.5050125112 \backslash \backslash$ Version=IA64L-G03RevC.02 $\backslash$ State $=2-A^{\prime} \backslash H F=-1$ $163.3466672 \backslash \mathrm{S} 2=0.753353 \backslash \mathrm{S} 2-1=0 . \backslash \mathrm{S} 2 \mathrm{~A}=0.750006 \backslash \mathrm{RMS} \mathrm{D}=6.765 \mathrm{e}-09 \backslash \mathrm{RMSF}=3.869$ $e-05 \backslash$ Dipole $=0.0002352,0 .,-0.0372733 \backslash P G=C S \quad[S G(H 1 S i 2), X(H 8 S i 2)] \backslash \backslash e$

\section{$\left(\mathrm{CH}_{3}\right)_{3} \mathrm{SiH}$}

$1 \backslash 1 \backslash G I N C-A C 32 \backslash P O p t \backslash R B 3 L Y P \backslash 6-31 G(d) \backslash C 3 H 10 S i 1 \backslash E X K 501 \backslash 30-M a r-2007 \backslash 1 \backslash \backslash \#$ B3 LYP $/ 6-31 \mathrm{G}(\mathrm{D}) \quad \mathrm{OPT}=(\mathrm{Z}-\mathrm{MATRIX}) \quad \mathrm{FREQ}=$ NORAMAN MAXDISK=134217728\\Me3SiHcore $\mathrm{C} 3 \mathrm{~V} \backslash \backslash 0,1 \backslash \mathrm{H} \backslash \mathrm{Si}, 1, \mathrm{~B} 1 \backslash \mathrm{C}, 2, \mathrm{~B} 2,1, \mathrm{~A} 1 \backslash \mathrm{C}, 2, \mathrm{~B} 2,1, \mathrm{~A} 1,3, \mathrm{D} 1,0 \backslash \mathrm{C}, 2, \mathrm{~B} 2,1, \mathrm{~A} 1,3,-\mathrm{D} 1,0$ $\widehat{\mathrm{H}}_{\mathrm{H}}, 3, \mathrm{~B} 3,2, \mathrm{~A} 2,1, \mathrm{D} 2, \mathrm{O} \backslash \mathrm{H}, 4, \mathrm{~B} 3,2, \mathrm{~A} 2,1, \mathrm{D} 2,0 \backslash \mathrm{H}, 5, \mathrm{~B} 3,2, \mathrm{~A} 2,1, \mathrm{D} 2,0 \backslash \mathrm{H}, 3, \mathrm{~B} 3,2, \mathrm{~A} 2$, $6, \mathrm{D} 3,0 \backslash \mathrm{H}, 4, \mathrm{~B} 3,2, \mathrm{~A} 2,7, \mathrm{D} 3, \mathrm{O} \backslash \mathrm{H}, 5, \mathrm{~B} 3,2, \mathrm{~A} 2,8, \mathrm{D} 3, \mathrm{O} \backslash \mathrm{H}, 3, \mathrm{~B} 4,2, \mathrm{~A} 3,6, \mathrm{D} 4,0 \backslash \mathrm{H}, 4, \mathrm{~B} 4$ $, 2, \mathrm{~A} 3,7, \mathrm{D} 4, \mathrm{O} \backslash \mathrm{H}, 5, \mathrm{~B} 4,2, \mathrm{~A} 3,8, \mathrm{D} 4, \mathrm{O} \backslash \backslash \mathrm{B} 1=1.49589283 \backslash \mathrm{B} 2=1.89296344 \backslash \mathrm{B} 3=1.0964$ $2011 \backslash \mathrm{B} 4=1.096987 \backslash \mathrm{A} 1=108.41655283 \backslash \mathrm{A} 2=111.30829925 \backslash \mathrm{A} 3=111.27903825 \backslash \mathrm{D} 2=59$ $.99552385 \backslash D 3=-119.99104765 \backslash D 4=120.00447617 \backslash D 1=120 . \backslash \backslash$ Version=IA64L-G03R evC.02 State $=1-\mathrm{A} 1 \backslash \mathrm{HF}=-409.8649361 \backslash \mathrm{RMSD}=5.503 \mathrm{e}-09 \backslash \mathrm{RMSF}=2.391 \mathrm{e}-05 \backslash \mathrm{Dipole}$ $=0 ., 0 ., 0.2413668 \backslash \mathrm{PG}=\mathrm{C} 03 \mathrm{~V} \quad[\mathrm{C} 3(\mathrm{Si} 1 \mathrm{H} 1), 3 \mathrm{SGV}(\mathrm{C} 1 \mathrm{H} 1), \mathrm{X}(\mathrm{H} 6)] \backslash \backslash \mathrm{C}$

\section{$\left(\mathrm{CH}_{3}\right)_{3} \mathrm{Si} \cdot$}

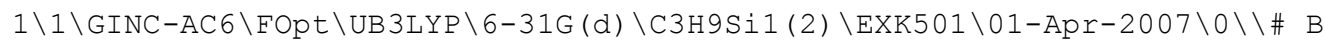
$3 \mathrm{LYP} / 6-31 \mathrm{G}(\mathrm{D})$ OPT $\mathrm{FREQ}=\mathrm{NORAMAN}$ MAXDISK=134217728 \\Me3Siradcore C3V $2 \backslash \backslash$ $0,2 \backslash \mathrm{Si}, 0 .,-0.000101772,0.4211090375 \backslash \mathrm{C}, 0 ., 1.8046106637,-0.17578 \overline{3} 222 \overline{7} \backslash \mathrm{C}$, $1.5628003465,-0.9022415482,-0.1761744691 \backslash C,-1.5628003465,-0.9022415482$ 
$,-0.1761744691 \backslash \mathrm{H}, 0.8843776443,2.3433459962,0.1828891071 \backslash \mathrm{H}, 1.5876009542$ $,-1.9373125656,0.1830105049 \backslash \mathrm{H},-2.4714387205,-0.4051937943,0.1820153006$ $\backslash \mathrm{H},-0.8843776443,2.3433459962,0.1828891071 \backslash \mathrm{H}, 2.4714387205,-0.405193794$ $3,0.1820153006 \backslash \mathrm{H},-1.5876009542,-1.9373125656,0.1830105049 \backslash \mathrm{H}, 0 ., 1.85823$ $18474,-1.2739172112 \backslash \mathrm{H}, 1.6090775127,-0.9296258576,-1.2743230871 \backslash \mathrm{H},-1.60$ $90775127,-0.9296258576,-1.2743230871 \backslash \backslash$ Version=IA 64 L-G03RevC.02 $\backslash$ State $=2$ $-A^{\prime} \backslash \mathrm{HF}=-409.210922 \backslash \mathrm{S} 2=0.751152 \backslash \mathrm{S} 2-1=0 . \backslash \mathrm{S} 2 \mathrm{~A}=0.750001 \backslash \mathrm{RMSD}=3.746 \mathrm{e}-09 \backslash \mathrm{RMS}$ $\mathrm{F}=2.633 \mathrm{e}-04 \backslash \mathrm{Dipole}=0 ., 0.0000398,-0.2631983 \backslash \mathrm{PG}=\mathrm{CS} \quad[\mathrm{SG}(\mathrm{C} 1 \mathrm{H} 1 \mathrm{Si} 1), \mathrm{X}(\mathrm{C} 2 \mathrm{H} 8)]$ $\backslash \backslash \mathrm{Q}$

\section{$\left(\mathrm{CH}_{3}\right)_{3} \mathrm{CH}$}

$1 \backslash 1 \backslash G I N C-A C 11 \backslash P O p t \backslash R B 3 L Y P \backslash 6-31 G(d) \backslash C 4 H 10 \backslash E X K 501 \backslash 01-A p r-2007 \backslash 1 \backslash \backslash \#$ B3LYP

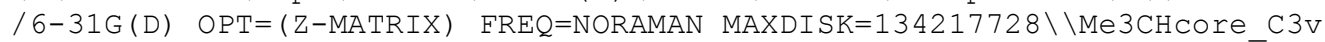
$\backslash \backslash 0,1 \backslash H \backslash C, 1, B 1 \backslash C, 2, B 2,1, A 1 \backslash C, 2, B 2,1, A 1,3, D 1,0 \backslash C, 2, B 2,1, A 1,3,-D 1,0 \backslash \bar{H}, 3$, $\mathrm{B} 3,2, \mathrm{~A} 2,1, \mathrm{D} 2, \mathrm{O} \backslash \mathrm{H}, 4, \mathrm{~B} 3,2, \mathrm{~A} 2,1, \mathrm{D} 2,0 \backslash \mathrm{H}, 5, \mathrm{~B} 3,2, \mathrm{~A} 2,1, \mathrm{D} 2,0 \backslash \mathrm{H}, 3, \mathrm{~B} 3,2, \mathrm{~A} 2,6, \mathrm{D} 3$, $0 \backslash \mathrm{H}, 4, \mathrm{~B} 3,2, \mathrm{~A} 2,7, \mathrm{D} 3,0 \backslash \mathrm{H}, 5, \mathrm{~B} 3,2, \mathrm{~A} 2,8, \mathrm{D} 3,0 \backslash \mathrm{H}, 3, \mathrm{~B} 4,2, \mathrm{~A} 3,6, \mathrm{D} 4,0 \backslash \mathrm{H}, 4, \mathrm{~B} 4,2, \mathrm{~A} 3$ $, 7, \mathrm{D} 4,0 \backslash \mathrm{H}, 5, \mathrm{~B} 4,2, \mathrm{~A} 3,8, \mathrm{D} 4,0 \backslash \backslash \mathrm{B} 1=1.10057031 \backslash \mathrm{B} 2=1.53539514 \backslash \mathrm{B} 3=1.09665779 \backslash$ $\mathrm{B} 4=1.09808204 \backslash \mathrm{A} 1=107.76975865 \backslash \mathrm{A} 2=111.3722561 \backslash \mathrm{A} 3=110.84595808 \backslash \mathrm{D} 2=60.236$ $14993 \backslash D 3=-120.40965145 \backslash D 4=119.80275482 \backslash D 1=120 . \backslash \backslash$ Version=IA $64 \mathrm{~L}-\mathrm{G} 03 \mathrm{RevC}$. $02 \backslash$ State $=1-A \backslash H F=-158.458812 \backslash R M S D=5.082 e-09 \backslash R M S F=1.098 e-05 \backslash D i p o l e=0 ., 0$. ,$-0.0335914 \backslash \mathrm{PG}=\mathrm{CO} 3 \quad[\mathrm{C} 3(\mathrm{C} 1 \mathrm{H} 1), \mathrm{X}(\mathrm{C} 3 \mathrm{H} 9)] \backslash \backslash \mathrm{Q}$

\section{$(\mathbf{H O}) \mathrm{H}_{2} \mathbf{P}(=\mathbf{O})$}

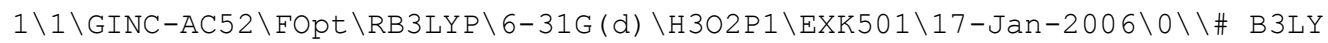
$\mathrm{P} / 6-31 \mathrm{G} *$ OPT $\mathrm{FREQ}=\mathrm{NORAMAN}$ MAXDISK $=134217728 \backslash \backslash \mathrm{H} 3 \mathrm{PO} 2 \mathrm{a} \backslash \backslash 0,1 \backslash \mathrm{H},-0.4284634$ $732,0.0840336582,-1.6022911425 \backslash P,-0.3159342264,0.1 \overline{0} 60422706,-0.1914268$ $277 \backslash 0,0.3467098454,1.3212112991,0.3474615458 \backslash \mathrm{H},-1.6233549684,-0.184813$ $2295,0.247372246 \backslash 0,0.3384560435,-1.3425139796,0.1656415122 \backslash \mathrm{H}, 1.3095047$ $274,-1.319433043,0.1214968486 \backslash \backslash$ Version=IA64L-G03RevC.02\State=1-A \HF=$493.6155029 \backslash \mathrm{RMSD}=3.462 e-09 \backslash \mathrm{RMSF}=1.343 e-04 \backslash \mathrm{Dipole}=-0.0742121,-0.8346251$ ,$-0.5924492 \backslash \mathrm{PG}=\mathrm{C} 01 \quad[\mathrm{X}(\mathrm{H} 302 \mathrm{P} 1)] \backslash \backslash \mathrm{Q}$

\section{$(\mathrm{HO}) \mathrm{HP}(=\mathbf{O}) \bullet$}

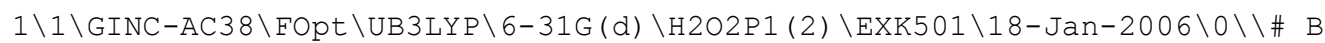

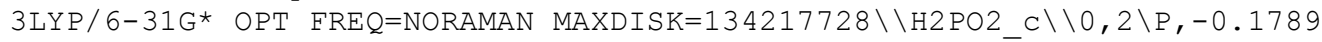
$140733,-0.0491782144,-0.3862809589 \backslash 0,1.29970628,0.188 \overline{1} 522444,-0.524284$ $8793 \backslash \mathrm{H},-0.9909448525,1.0475976013,-0.8041142404 \backslash 0,-0.8005303588,-0.130$ $811567,1.1375714924 \backslash \mathrm{H},-0.3187514178,-0.7686498051,1.6920357197 \backslash \backslash$ Versio $\mathrm{n}=\mathrm{IA} 64 \mathrm{~L}-\mathrm{G} 03 \mathrm{RevC} .02 \backslash \mathrm{State}=2-\mathrm{A} \backslash \mathrm{HF}=-492.9774595 \backslash \mathrm{S} 2=0.754202 \backslash \mathrm{S} 2-1=0 . \backslash \mathrm{S} 2 \mathrm{~A}=0$ $.750009 \backslash \mathrm{RMSD}=4.713 \mathrm{e}-09 \backslash \mathrm{RMSF}=5.846 \mathrm{e}-05 \backslash \mathrm{Dipole}=-0.7004656,-0.3874243,0.3$ $433488 \backslash \mathrm{PG}=\mathrm{CO} 1 \quad[\mathrm{X}(\mathrm{H} 2 \mathrm{O} 2 \mathrm{P} 1)] \backslash \backslash \mathrm{Q}$

\section{$\left(\mathrm{CH}_{3} \mathrm{CH}_{2}\right)_{2} \mathrm{HP}(=\mathrm{O})$}

$1 \backslash 1 \backslash G I N C-A C 57 \backslash F O p t \backslash R B 3 L Y P \backslash 6-31 G(d) \backslash C 4 H 1101 P 1 \backslash E X K 501 \backslash 30-M a r-2007 \backslash 0 \backslash \backslash \# \quad B$ 3LYP/6-31G(D) OPT EREQ=NORAMAN MAXDISK=134217728 MAXDISK=134217728 MAX

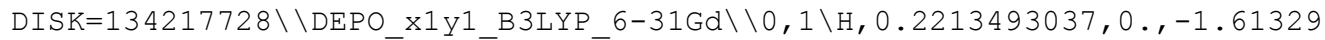
$84923 \backslash \mathrm{P}, 0.2296235491,0 .,-\overline{0} .1861 \overline{1} 91603 \backslash 0,1.5989418421,0 ., 0.4298381432 \backslash \mathrm{C}$ $,-0.8132990909,1.4610967429,0.2035670571 \backslash C,-0.8132990909,-1.4610967429$ $, 0.2035670571 \backslash \mathrm{C},-0.1196181743,2.7818154986,-0.1570707146 \backslash \mathrm{C},-0.11961817$ $43,-2.7818154986,-0.1570707146 \backslash \mathrm{H},-1.0316498594,1.4239445787,1.27846463$ $62 \backslash \mathrm{H},-1.0316498594,-1.4239445787,1.2784646362 \backslash \mathrm{H},-1.7701285473,1.356777$ $8122,-0.3258299267 \backslash \mathrm{H},-1.7701285473,-1.3567778122,-0.3258299267 \backslash \mathrm{H}, 0.843$ $9400677,2.8628825256,0.3544479268 \backslash \mathrm{H}, 0.8439400677,-2.8628825256,0.35444$ $79268 \backslash \mathrm{H},-0.7386917835,3.637669645,0.1322973016 \backslash \mathrm{H},-0.7386917835,-3.6376$ $69645,0.1322973016 \backslash \mathrm{H}, 0.0654150748,2.8516062349,-1.2351676176 \backslash \mathrm{H}, 0.06541$ $50748,-2.8516062349,-1.2351676176 \backslash \backslash$ Version=IA64L-G03RevC.02 $\backslash$ State $=1-A^{\prime}$ $\backslash \mathrm{HF}=-575.6484563 \backslash \mathrm{RMSD}=2.229 \mathrm{e}-09 \backslash \mathrm{RMSF}=4.986 \mathrm{e}-05 \backslash \mathrm{Dipole}=-1.5278381,0 .,-0$ $.354976 \backslash \mathrm{PG}=\mathrm{CS} \quad[\mathrm{SG}(\mathrm{H} 1 \mathrm{O} 1 \mathrm{P} 1), \mathrm{X}(\mathrm{C} 4 \mathrm{H} 10)] \backslash \backslash @$

\section{$\left(\mathrm{CH}_{3} \mathrm{CH}_{2}\right)_{2} \mathbf{P}(=\mathrm{O}) \bullet$}

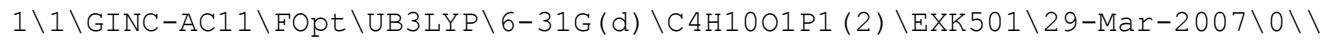
\# B3LYP/6-31G(D) OPT FREQ=NORAMAN MAXDISK=134217728\\DEPOrad_x1Y1_B3LY 


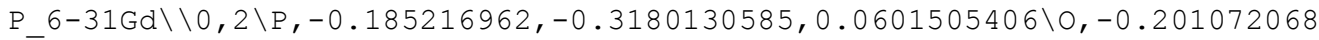
$4,-0.345235894,1.5810827937 \backslash C, 1.5312383933,-0.2960795087,-0.6551414275$ $\backslash C,-1.0130644295,1.1857717125,-0.6551414275 \backslash C, 2.4008864955,-1.43967905$ $99,-0.1219790118 \backslash \mathrm{C},-2.4368579399,1.3779169571,-0.1219790118 \backslash \mathrm{H}, 1.977866$ $3408,0.6770951776,-0.407239558 \backslash \mathrm{H},-0.3869760196,2.0544251512,-0.4072395$ $58 \backslash \mathrm{H}, 1.4364567088,-0.3406926269,-1.747552783 \backslash \mathrm{H},-1.0051019259,1.0813183$ $901,-1.747552783 \backslash \mathrm{H}, 2.4511503999,-1.409307618,0.9704241096 \backslash \mathrm{H},-2.4352420$ $181,1.4366219259,0.9704241096 \backslash \mathrm{H}, 3.4202487116,-1.3654233335,-0.51631623$ $7 \backslash \mathrm{H},-2.8752395568,2.3011909134,-0.516316237 \backslash \mathrm{H}, 1.9971179551,-2.41580950$ $78,-0.4120531248 \backslash \mathrm{H},-3.0866647355,0.5450839523,-0.4120531248 \backslash \backslash$ Version $=\mathrm{I}$ A 64 L-G03RevC.02 \State $=2-A^{\prime} \backslash H F=-575.0117831 \backslash S 2=0.754237 \backslash S 2-1=0 . \backslash S 2 A=0.7$ $50006 \backslash \mathrm{RMS} D=7.367 \mathrm{e}-09 \backslash \mathrm{RMSF}=2.154 \mathrm{e}-05 \backslash \mathrm{Dipole}=0.264429,0.4540183,-1.28143$ $2 \backslash P G=C S \quad[S G(O 1 P 1), X(C 4 H 10)] \backslash \backslash @$

\section{$\left(\mathrm{CH}_{3}\right)_{2} \mathrm{HP}(=\mathbf{O})$}

$1 \backslash 1 \backslash G I N C-A C 6 \backslash F O p t \backslash R B 3 L Y P \backslash 6-31 G(d) \backslash C 2 H 701 P 1 \backslash E X K 501 \backslash 01-A p r-2007 \backslash 0 \backslash \backslash \#$ B3L YP/6-31G (D) OPT FREQ=NORAMAN MAXDISK=134217728 \\Me2HP=Ocore $\backslash \backslash 0,1 \backslash \mathrm{H}, 1.4$ $018795457,-0.9590512791,0 . \backslash P, 0.1314745596,-0.3157272056,0 . \backslash 0,-1.047475$ $3038,-1.240099839,0 . \backslash \mathrm{C}, 0.271395521,0.7933017134,1.4490755701 \backslash \mathrm{C}, 0.27139$ $5521,0.7933017134,-1.4490755701 \backslash \mathrm{H},-0.5925538813,1.4647363963,1.4838167$ $537 \backslash \mathrm{H},-0.5925538813,1.4647363963,-1.4838167537 \backslash \mathrm{H}, 1.1897308174,1.389598$ $1889,1.4056186667 \backslash \mathrm{H}, 1.1897308174,1.3895981889,-1.4056186667 \backslash \mathrm{H}, 0.277352$ $1827,0.1937341722,2.3642365111 \backslash \mathrm{H}, 0.2773521827,0.1937341722,-2.36423651$ $11 \backslash \backslash$ Version=IA6 4L-G03RevC.02 \State $=1-A \cdot \backslash H F=-497.0229113 \backslash$ RMSD $=2.813 e-09$ $\backslash \mathrm{RMSF}=1.164 \mathrm{e}-05 \backslash \mathrm{Dipole}=1.0907036,1.2348405,0 . \backslash \mathrm{PG}=\mathrm{CS} \quad[\mathrm{SG}(\mathrm{H} 1 \mathrm{O} 1 \mathrm{P} 1), \mathrm{X}(\mathrm{C} 2 \mathrm{H} 6$ ) $] \backslash \backslash Q$

\section{$\left(\mathrm{CH}_{3} \mathrm{CH}_{2}\right)_{2} \mathbf{P}(=\mathbf{O}) \bullet$}

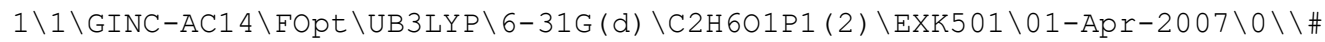
B3LYP/6-31G(D) OPT FREQ=NORAMAN MAXDISK=134217728 \\Me2P=Orad_core $\backslash \backslash 0$, $2 \backslash \mathrm{P},-0.2445365542,0.3218770919,0 . \backslash 0,0.8366884343,1.3879226032,0 . \backslash \mathrm{C},-0$. $1730190288,-0.8103353758,1.4593536989 \backslash \mathrm{C},-0.1730190288,-0.8103353758,-1$ $.4593536989 \backslash \mathrm{H}, 0.7672767217,-1.3745721724,1.4603986444 \backslash \mathrm{H}, 0.7672767217,-$ $1.3745721724,-1.4603986444 \backslash \mathrm{H},-1.014272388,-1.5107029724,1.4353779658 \backslash \mathrm{H}$ $,-1.014272388,-1.5107029724,-1.4353779658 \backslash \mathrm{H},-0.2276197418,-0.218481202$ $7,2.3769308625 \backslash \mathrm{H},-0.2276197418,-0.2184812027,-2.3769308625 \backslash \backslash$ Version=IA $64 \mathrm{~L}-\mathrm{G} 03$ RevC.02 $\backslash$ State $=2-\mathrm{A}^{\prime} \backslash \mathrm{HF}=-496.3857192 \backslash \mathrm{S} 2=0.754326 \backslash \mathrm{S} 2-1=0 . \backslash \mathrm{S} 2 \mathrm{~A}=0.75$ $0006 \backslash \mathrm{RMSD}=8.534 \mathrm{e}-09 \backslash \mathrm{RMSF}=4.859 \mathrm{e}-05 \backslash \mathrm{Dipole}=-0.6164262,-1.3206206,0 . \backslash \mathrm{PG}=$ $\mathrm{CS} \quad[\mathrm{SG}(\mathrm{O} 1 \mathrm{P} 1), \mathrm{X}(\mathrm{C} 2 \mathrm{H} 6)] \backslash \backslash @$

\section{$\left(\mathrm{CH}_{3}\right) \mathrm{CH}(\mathrm{Cl}) \mathrm{C}(\mathrm{H}) \cdot \mathrm{CH}(\mathrm{Cl}) \mathrm{CH}_{2} \mathrm{CH}_{2} \mathrm{Cl}$}

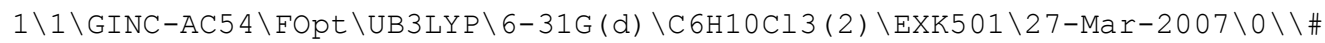
B3LYP/6-31G(D) OPT FREQ=NORAMAN MAXDISK=134217728\\midchaintri-betaCl $-S M \_a 1 b 1 B B 3 L Y P \backslash \backslash 0,2 \backslash H, 3.071414794,1.0066742785,0.1457342329 \backslash \mathrm{C}, 2.804472$ $195,0.07 \overline{7} 6998783,0.6502199694 \backslash \mathrm{Cl}, 3.0737945709,-1.2602159886,-0.5533685$ $532 \backslash \mathrm{C}, 1.3713657812,0.1051857915,1.1655020006 \backslash \mathrm{H}, 3.50232658,-0.104813426$ $5,1.4688137089 \backslash \mathrm{C}, 0.2960716654,0.3993594758,0.123686928 \backslash \mathrm{H}, 1.3154959371$, $0.8567577974,1.9641094266 \backslash \mathrm{H}, 1.1292643984,-0.8662879788,1.6152550642 \backslash \mathrm{H}$, $0.4029711656,-0.2470443035,-0.7491915017 \backslash \mathrm{Cl}, 0.6078960683,2.1289675923$, $-0.6117884032 \backslash \mathrm{C},-1.0761034496,0.3968266959,0.6495091879 \backslash \mathrm{C},-2.244611446$ $8,0.0901758318,-0.1911863562 \backslash \mathrm{Cl},-2.5232895804,-1.7832076741,-0.1034456$ $294 \backslash \mathrm{H},-2.0261129192,0.2452383954,-1.2496055504 \backslash \mathrm{C},-3.5334720445,0.78373$ $57446,0.2202646271 \backslash \mathrm{H},-4.3694412165,0.4585894721,-0.4042188214 \backslash \mathrm{H},-3.409$ $7607973,1.8673355945,0.1042340548 \backslash \mathrm{H},-3.7766576071,0.5709498047,1.26598$ $1342 \backslash \mathrm{H},-1.2386545404,0.6404530555,1.6971538617 \backslash \backslash$ Version=IA64L-G0 3RevC. $02 \backslash \mathrm{State}=2-\mathrm{A} \backslash \mathrm{HF}=-1615.2151644 \backslash \mathrm{S} 2=0.755926 \backslash \mathrm{S} 2-1=0 . \backslash \mathrm{S} 2 \mathrm{~A}=0.750028 \backslash \mathrm{RMSD}=7$. $308 e-09 \backslash$ RMSF $=1.727 e-05 \backslash \mathrm{Dipole}=-0.2512026,0.6827983,0.9441968 \backslash \mathrm{PG}=\mathrm{C} 01 \quad[\mathrm{X}$ $(\mathrm{C} 6 \mathrm{H} 10 \mathrm{Cl3})] \backslash \backslash \mathrm{e}$

\section{$\left(\mathrm{CH}_{3}\right) \mathrm{CH}(\mathrm{Cl}) \mathrm{CH}=\mathrm{CHCH}_{2} \mathrm{CH}_{2} \mathrm{Cl}$}

$1 \backslash 1 \backslash$ GINC-AC2 $\backslash$ FOpt $\backslash$ RB 3LYP \6-31G (d) \C6H10C12 YP/6-31G(D) OPT FREQ=NORAMAN MAXDISK=134217728\\midchaintri-betaCl-pro

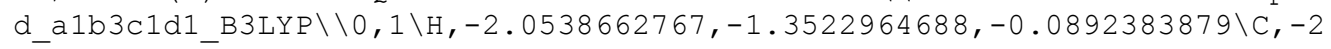


$.4275736139,-0.4263901738,0.3511446431 \backslash \mathrm{Cl},-4.1401584839,-0.2283801821$, $-0.2268548715 \backslash \mathrm{C},-1.5615832966,0.7713053719,-0.046349605 \backslash \mathrm{H},-2.480745335$ $7,-0.5457396568,1.4355441848 \backslash C,-0.1662427768,0.6250727735,0.5001115591$ $\backslash \mathrm{H},-2.0271397879,1.6856276927,0.3442227449 \backslash \mathrm{H},-1.5395155043,0.858530039$ $2,-1.1378500197 \backslash \mathrm{C}, 0.932771852,0.478037258,-0.245637101 \backslash \mathrm{C}, 2.3129626422$, $0.3640770666,0.317200345 \backslash \mathrm{Cl}, 2.9757775108,-1.325790957,-0.065155789 \backslash \mathrm{H}, 2$ $.286008599,0.3988471543,1.4086486263 \backslash C, 3.2851708314,1.4017732278,-0.23$ $36071333 \backslash \mathrm{H}, 4.2872011516,1.2591535478,0.1803243068 \backslash \mathrm{H}, 2.9326045713,2.404$ $7545153,0.0354984886 \backslash \mathrm{H}, 3.3479427062,1.3384776731,-1.3245299486 \backslash \mathrm{H}, 0.859$ $7526237,0.4581714694,-1.3332840285 \backslash H,-0.0708000351,0.6321302542,1.5876$ $690146 \backslash \backslash$ Version=IA64L-G03RevC .02 \State $=1-A \backslash H F=-1155.0476519 \backslash$ RMSD $=3.397$ $\mathrm{e}-09 \backslash \mathrm{RMSF}=1.706 \mathrm{e}-05 \backslash \mathrm{Dipole}=0.4622803,0.8357617,0.3835309 \backslash \mathrm{PG}=\mathrm{C} 01 \quad[\mathrm{X}(\mathrm{C} 6 \mathrm{H}$ $10 \mathrm{C} 12)] \backslash \backslash 0$

\section{$\left(\mathrm{CH}_{3}\right) \mathrm{CH}(\mathrm{Cl}) \mathrm{CH}=\mathrm{CClCH}_{2} \mathrm{CH}_{2} \mathrm{Cl}$}

$1 \backslash 1 \backslash G I N C-A C 58 \backslash F O p t \backslash R B 3 L Y P \backslash 6-31 G(d) \backslash C 6 H 9 C 13 \backslash E X K 501 \backslash 26-M a r-2007 \backslash 0 \backslash \backslash \#$ B3L YP/6-31G(D) OPT FREQ=NORAMAN MAXDISK=134217728 \\prod betaHelim start B $3 \mathrm{LYP} \backslash \backslash 0,1 \backslash \mathrm{H}, 1.99500826,-1.2312092047,-0.931139283 \backslash \mathrm{C}, \overline{2} .4163186,-0.353 \overline{5} 1$ $0596,-0.4381297886 \backslash \mathrm{Cl}, 4.0802805623,-0.8116981106,0.1213565679 \backslash \mathrm{C}, 1.5516$ $445486,0.0890000024,0.7466869422 \backslash \mathrm{H}, 2.5397966625,0.4458566351,-1.169429$ $0045 \backslash \mathrm{C}, 0.1586088538,0.4723342909,0.3198095389 \backslash \mathrm{H}, 2.0354794445,0.9331803$ $821,1.2509499295 \backslash \mathrm{H}, 1.4820338412,-0.7312124637,1.4687450335 \backslash \mathrm{C},-0.944392$ $6044,-0.2269182569,0.6054246368 \backslash C,-2.3527967956,0.0929277266,0.2257317$ $033 \backslash \mathrm{Cl},-2.9594127175,-1.2445461934,-0.9004083086 \backslash \mathrm{H},-2.3998913278,0.997$ $6496752,-0.3791931755 \backslash \mathrm{C},-3.2887429718,0.1901895774,1.4259942805 \backslash \mathrm{H},-4.3$ $15640544,0.3794153816,1.1015127306 \backslash \mathrm{H},-2.9660392066,1.0176694921,2.0691$ $182177 \backslash \mathrm{H},-3.2741567626,-0.7330905255,2.0136461309 \backslash \mathrm{H},-0.8086017831,-1.1$ $330564286,1.1938985552 \backslash \mathrm{Cl}, 0.0831423688,1.9662831623,-0.6286666718 \backslash \backslash \mathrm{Ver}$ sion=IA64L-G03RevC.02 \State $=1-A \backslash H F=-1614.6434046 \backslash$ RMSD $=3.790 e-09 \backslash$ RMSF $=1$ $.377 e-05 \backslash \mathrm{Dipole}=-0.447075,0.3277631,0.7000432 \backslash \mathrm{PG}=\mathrm{C} 01 \quad[\mathrm{X}(\mathrm{C} 6 \mathrm{H} 9 \mathrm{Cl} 3)] \backslash \backslash @$

\section{$\left(\mathrm{CH}_{3}\right) \mathrm{C}(\mathrm{H}) \cdot \mathrm{CH}_{3}$}

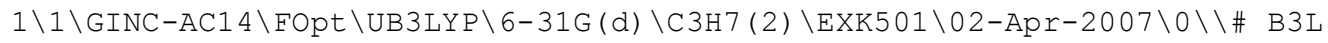
YP/6-31G(D) OPT FREQ=NORAMAN MAXDISK=134217728\\midchaintri-betaCl-SM alb1 B3LYP alphacore $2 \backslash \backslash 0,2 \backslash C,-0.0004167261,0.5344863575,0 . \backslash \mathrm{H}, 0.27735 \overline{4}$ $0653,1.586 \overline{1} 958564,0 . \backslash \mathrm{C},-0.015699752,-0.1971297645,1.3010857006 \backslash \mathrm{C},-0.01$ $5699752,-0.1971297645,-1.3010857006 \backslash \mathrm{H},-0.2479791894,0.4667304682,2.141$ $6436582 \backslash \mathrm{H},-0.2479791894,0.4667304682,-2.1416436582 \backslash \mathrm{H}, 0.9587646647,-0.6$ $688558619,1.5275654021 \backslash \mathrm{H}, 0.9587646647,-0.6688558619,-1.5275654021 \backslash \mathrm{H},-0$ $.7540138179,-1.0116530199,1.297162033 \backslash \mathrm{H},-0.7540138179,-1.0116530199,-1$ $.297162033 \backslash \backslash$ Version=IA64L-G03RevC.02\State $=2-A \cdot \backslash H F=-118.4781544 \backslash S 2=0.7$ $54003 \backslash \mathrm{S} 2-1=0 . \backslash \mathrm{S} 2 \mathrm{~A}=0.750011 \backslash \mathrm{RMSD}=4.197 \mathrm{e}-09 \backslash \mathrm{RMSF}=3.357 \mathrm{e}-06 \backslash \mathrm{Dipole}=0.0669$ $559,-0.0433416,0 . \backslash \mathrm{PG}=\mathrm{CS} \quad[\mathrm{SG}(\mathrm{C} 1 \mathrm{H} 1), \mathrm{X}(\mathrm{C} 2 \mathrm{H} 6)] \backslash \backslash @$

\section{$\left(\mathrm{CH}_{3}\right) \mathrm{CH}=\mathrm{CH}_{2}$}

$1 \backslash 1 \backslash G I N C-A C 30 \backslash F O p t \backslash R B 3 L Y P \backslash 6-31 G(d) \backslash C 3 H 6 \backslash E X K 501 \backslash 01-A p r-2007 \backslash 0 \backslash \backslash \#$ B3LYP / 6-31G (D) OPT FREQ=NORAMAN MAXDISK=134217728 \prod betaHelim start B3LY P alphacore $\backslash \backslash 0,1 \backslash \mathrm{C},-1.2056287384,-0.4590545688,0 . \overline{1} 742954696 \backslash \mathrm{C}, 0.0 \overline{8} 3307$ $9 \overline{9} 39,-0.4369778636,-0.1660137524 \backslash C, 0.9965953927,0.7467807423,-0.023514$ $214 \backslash \mathrm{H}, 0.533691091,-1.3390544368,-0.5831509242 \backslash \mathrm{H},-1.8169737498,-1.34860$ $97454,0.0477265355 \backslash \mathrm{H},-1.7004510785,0.4147015734,0.5943655205 \backslash \mathrm{H}, 1.85119$ $76252,0.5133035756,0.625951856 \backslash \mathrm{H}, 1.4142364514,1.0454402821,-0.99464795$ $85 \backslash \mathrm{H}, 0.4726517715,1.6097288916,0.4011499518 \backslash \backslash$ Version=IA64L-G03RevC.02\} State $=1-\mathrm{A} \backslash \mathrm{HF}=-117.9075561 \backslash \mathrm{RMS} \mathrm{D}=6.982 \mathrm{e}-0 \mathrm{9} \backslash \mathrm{RMSF}=3.269 \mathrm{e}-05 \backslash \mathrm{Dipole}=0.13199$ $84,0.0398354,-0.0222631 \backslash P G=C 01[X(\mathrm{C} 3 \mathrm{H} 6)] \backslash \backslash \mathrm{Q}$

\section{$\left(\mathrm{CH}_{3}\right) \mathrm{CH}(\mathrm{Cl}) \mathrm{C}(\mathrm{H}) \cdot \mathrm{CH}_{3}$}

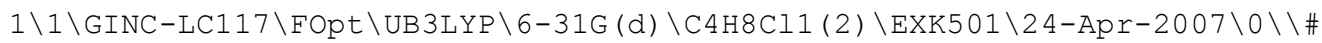
B3LYP/6-31G(D) OPT FREQ=NORAMAN MAXDISK=134217728\\midchaintri-betaCl -SM a1b1 newalphacore B3LYP \\0,2\H,-2.1720583155,-1.4319959335, 0.16379 $76206 \backslash \mathrm{C},-1.1179047509,-1.5631871928,0.4203071835 \backslash \mathrm{C},-0.3869493645,-0.23$ $08837149,0.435873738 \backslash \mathrm{H},-0.6666047071,-2.2469640892,-0.3052667387 \backslash \mathrm{H},-1$. 
$0544497015,-2.022282054,1.4149085066 \backslash \mathrm{H},-0.8754551487,0.4970101242,1.08$ $6904076 \backslash \mathrm{Cl},-0.6753400769,0.5916780072,-1.2953544831 \backslash \mathrm{C}, 1.0528769716,-0$. $2981529181,0.6696644285 \backslash \mathrm{C}, 1.8580403314,0.8796539861,1.0997068952 \backslash \mathrm{H}, 2.7$ $440228078,0.5735445563,1.6684058678 \backslash \mathrm{H}, 1.2715487474,1.5728902323,1.7141$ $468288 \backslash \mathrm{H}, 2.2200843407,1.4509195741,0.2286847248 \backslash \mathrm{H}, 1.5773141582,-1.1762$ $294945,0.2961318558 \backslash \backslash$ Version=IA32L-G03RevC.02 $\backslash$ State $=2-A \backslash H F=-617.395337$ $8 \backslash \mathrm{S} 2=0.755549 \backslash \mathrm{S} 2-1=0 . \backslash \mathrm{S} 2 \mathrm{~A}=0.750022 \backslash \mathrm{RMSD}=3.187 \mathrm{e}-09 \backslash \mathrm{RMSF}=3.720 \mathrm{e}-06 \backslash \mathrm{Dipol}$ $\mathrm{e}=0.3683039,-0.4042803,0.9366454 \backslash \mathrm{PG}=\mathrm{C} 01 \quad[\mathrm{X}(\mathrm{C} 4 \mathrm{H} 8 \mathrm{Cl} 1)] \backslash \backslash @$

\section{$E-\left(\mathrm{CH}_{3}\right) \mathrm{CH}=\mathrm{CHMe}$}

$1 \backslash 1 \backslash G I N C-L C 19 \backslash F O p t \backslash R B 3 L Y P \backslash 6-31 G(d) \backslash C 4 H 8 \backslash E X K 501 \backslash 24-A p r-2007 \backslash 0 \backslash \backslash \#$ B3LYP / 6-31G (D) OPT FREQ=NORAMAN MAXDISK=134217728\\midchaintri-betaCl-prod a

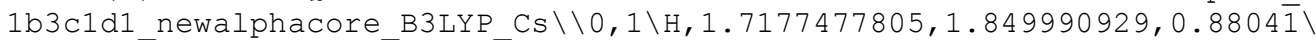
$\mathrm{H}, 1.7177477805,1.849990929,-0.88041 \backslash \mathrm{C}, 1.0986102994,1.6293027244,0 . \backslash \mathrm{C}, 0$ $.6372052415,0.1996102129,0 . \backslash \mathrm{H}, 0.2521234047,2.3247265731,0 . \backslash \mathrm{C},-0.637181$ $0643,-0.1996279742,0 . \backslash \mathrm{C},-1.0986204971,-1.6292828245,0 . \backslash \mathrm{H},-1.7177601168$ $,-1.8499570496,0.88041 \backslash \mathrm{H},-1.7177601168,-1.8499570496,-0.88041 \backslash \mathrm{H},-0.252$ $177184,-2.3247491071,0 . \backslash \mathrm{H},-1.4232787731,0.5585413845,0 . \backslash \mathrm{H}, 1.4232830504$ $,-0.5585693435,0 . \backslash \backslash$ Version=IA32L-G03RevC.02 $\backslash$ State=1-A' $\backslash H F=-157.2269061$ $\backslash \mathrm{RMSD}=1.863 \mathrm{e}-09 \backslash \mathrm{RMSF}=1.560 \mathrm{e}-05 \backslash \mathrm{Dipole}=0.0000114,-0.0000001,0 . \backslash \mathrm{PG}=\mathrm{CS} \quad[\mathrm{S}$ $\mathrm{G}(\mathrm{C} 4 \mathrm{H} 4), \mathrm{X}(\mathrm{H} 4)] \backslash \backslash \mathrm{Q}$

\section{$\mathrm{Z}-\left(\mathrm{CH}_{3}\right) \mathrm{CH}=\mathrm{CMeCl}$}

$1 \backslash 1 \backslash G I N C-L C 87 \backslash F O p t \backslash R B 3 L Y P \backslash 6-31 G(d) \backslash C 4 H 7 C 11 \backslash E X K 501 \backslash 24-A p r-2007 \backslash 0 \backslash \backslash \#$ B3L YP/6-31G(D) OPT FREQ=NORAMAN MAXDISK=134217728 \\prod betaHelim start $n$ ewalphacore B3LYP Cs $\backslash \backslash 0,1 \backslash \mathrm{H}, 2.4124990192,0.021694701 \overline{3}, 0.88297 \backslash \overline{\mathrm{H}}, 2.41 \overline{2} 4$ $990192,0.02 \overline{1} 69470 \overline{1} 3,-0.88297 \backslash \mathrm{C}, 1.9469650512,0.4753536702,0 . \backslash \mathrm{C}, 0.463493$ $0874,0.2562642709,0 . \backslash \mathrm{H}, 2.170077124,1.5470807348,0 . \backslash \mathrm{C},-0.4718819206,1.2$ $086019114,0 . \backslash \mathrm{C},-1.9623301142,1.0561677895,0 . \backslash \mathrm{H},-2.4007350573,1.5431096$ $386,0.88108 \backslash \mathrm{H},-2.4007350573,1.5431096386,-0.88108 \backslash \mathrm{H},-2.2671785227,0.00$ $75231409,0 . \backslash \mathrm{H},-0.0916097341,2.2304685635,0 . \backslash \mathrm{Cl}, 0.0181019593,-1.4643022$ $839,0 . \backslash \backslash$ Version=IA32L-G03RevC.02 \State $=1-A^{\prime} \backslash H F=-616.826253 \backslash$ RMSD $=3.321 \mathrm{e}$ $-09 \backslash \mathrm{RMSF}=3.100 e-05 \backslash \mathrm{Dipole}=-0.2534118,0.6714406,0 . \backslash \mathrm{PG}=\mathrm{CS} \quad[\mathrm{SG}(\mathrm{C} 4 \mathrm{H} 3 \mathrm{Cl} 1), \mathrm{X}$ $(\mathrm{H} 4)] \backslash \backslash \mathrm{Q}$ 